\title{
Der Einfluss jugendlicher Sozialisationserfahrungen auf ausgewählte Aspekte der politischen I dentität im Erwachsenenalter
}

Dissertation zur Erlangung des sozialwissenschaftlichen Doktorgrades der Sozialwissenschaftlichen Fakultät der Georg-August-Universität Göttingen

\author{
vorgelegt \\ von \\ Anja Mays \\ aus Köln
}

Göttingen 2008 
1. Gutachter: Prof. Dr. Steffen-Matthias Kühnel

2. Gutachter: Prof. Dr. Rainer Watermann

Tag der mündlichen Prüfung: 4.2.08 


\section{Inhalt}

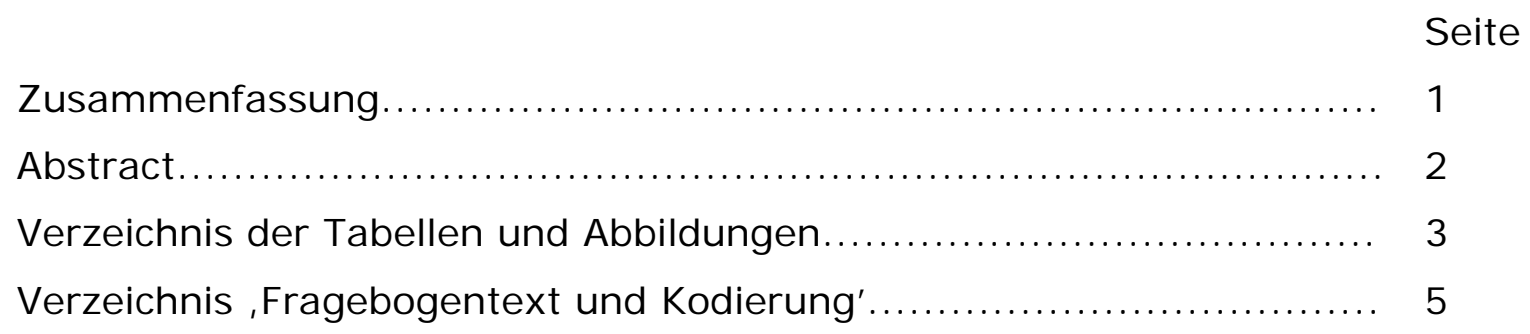

$1 \quad$ Einleitung und Fragestellung. 7

2 Theoretischer Hintergrund der politischen Sozialisation.

2.1 Begriff und Gegenstand der politischen Sozialisation -

Politische Sozialisation als Lernprozess....................... 11

2.2 Die Bedeutung der politischen Sozialisation für die Demokratie 12

2.3 Politische Identitätsbildung als Entwicklungsaufgabe des

Jugendalters............................................. 14

2.4 Dimensionen der politischen Identitätsbildung................. 15

2.4.1 Die affektiv-motivationale Dimension.......................... 15

2.4.2 Die kognitive Dimension................................... 16

2.4.3 Die behaviorale Dimension................................... 16

2.5 Latente und manifeste politische Sozialisation.................. 17

2.6 Modelle und Theorien der Politischen Sozialisation................. 20

2.6.1 Das ,Primacy Principle'-Modell.............................. 23

2.6.2 Das ,Intermediate Period'-Modell.............................. 24

2.6.3 Das ,Recency'-Model......................................... 25

2.6.4 Das Modell der politischen Sozialisation nach Weiß.............. 26

3 Politische Sozialisationsinstanzen im Lebensverlauf .............. 29

3.1 Politische Sozialisationsinstanzen............................. 29

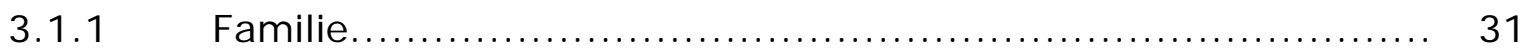

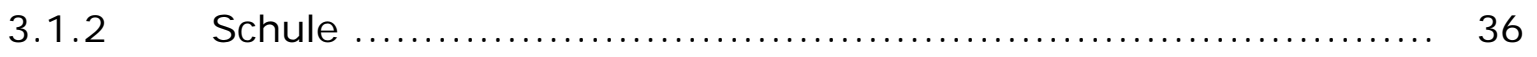

3.1.3 Peer Group............................................... 42

3.1.4 Medien.................................................... 44

3.1.5 Spätere Sozialisationseinflüsse.......................... 45 
$4 \quad$ Inhalte der politischen Identität: Politisches Interesse und ertorientierungen............................................ 48

$4.1 \quad$ Politisches Interesse........................................... 48

4.1.1 Determinanten des politischen Interesses..................... 50

4.1.2 Individuelle Entwicklung und Stabilität des politischen Interesses im Lebensverlauf.................................. 54

$4.2 \quad$ Gesellschaftspolitische Werte.................................. 58

4.2.1 Die These vom postmaterialistischen Wertewandel nach

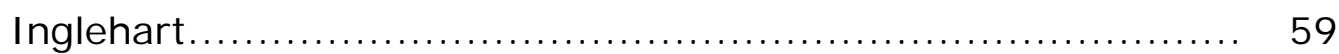

4.2.2 Empirische Befunde zur Postmaterialismusthese................. 63

4.2.3 Individuelle Entwicklung und Determinanten der politischen Werte im Lebensverlauf.......................................... 64

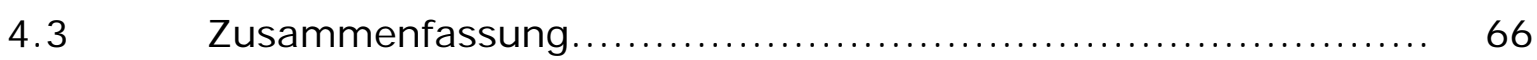

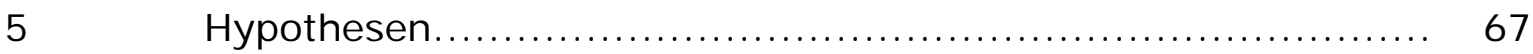

$5.1 \quad$ Hypothesen zum politischen Interesse....................... 67

$5.2 \quad$ Hypothesen zu Wertorientierungen........................... 73

$6 \quad$ Daten und Methode........................................... 77

$6.1 \quad$ Überblick über die Analysestrategie............................ 77

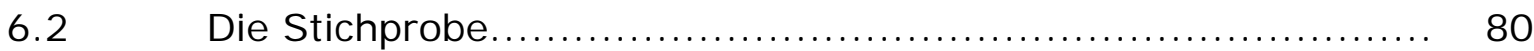

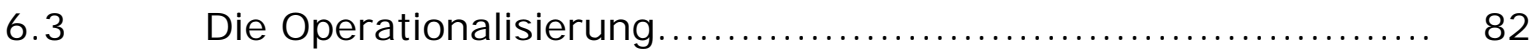

6.3.1 Die Messung der abhängigen Variablen........................ 82

6.3.1.1 Politisches Interesse....................................... 82

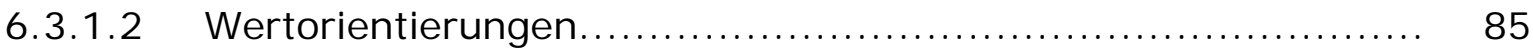

6.3.2 Die Messung der unabhängigen Variablen....................... 86

6.3.2.1 Sozio-ökonomischer Status des Elternhauses..................... 86

6.3.2.2 Kognitive Kompetenz in der Jugend........................... 87

6.3.2.3 Latente politische Sozialisation im Elternhaus.................... 89

6.3.2.4 Latente politische Sozialisation in der Schule.................... 91

6.3.2.5 Manifeste politische Sozialisation in der Schule.................. 92

6.3.2.6 Weitere manifeste Sozialisationserfahrungen in Jugend: Politische Partizipation in der Jugend......................... 94

6.3.2.7 Sekundäre Sozialisationserfahrungen.......................... 96 
6.4 Besonderheiten der Untersuchungsstichprobe und

Panelmortalität.................................................. 98

6.4.1 Besonderheiten der Untersuchungsstichprobe.................. 98

6.4.2 Panelausfälle............................................... 101

6.5 Übersicht über die verwendeten Variablen und Konstrukte....... 105

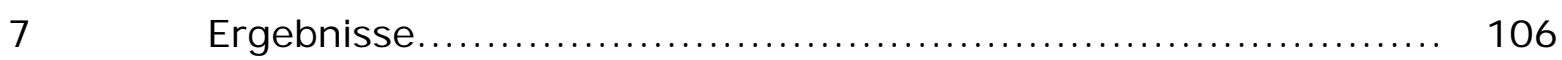

7.1 Stabilität der Zielvariablen..................................... 106

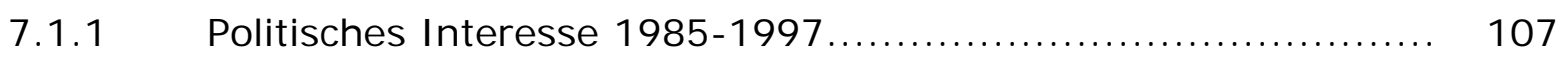

7.1.1.1 Die Randverteilungen von, subjektivem politischen Interesse' und ,Wichtigkeit von Politik' 1985-1997....................... 107

7.1.1.2 Zur individuellen Stabilität des politischen Interesses

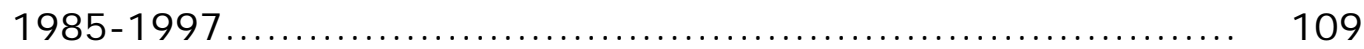

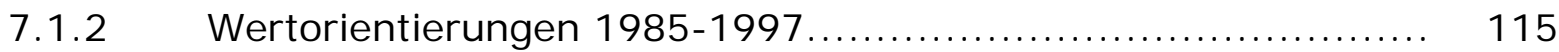

7.1.2.1 Die Randverteilungen der Wertorientierungen 1985-1997....... 115

7.1.2.2 Zur individuellen Stabilität der Wertorientierungen

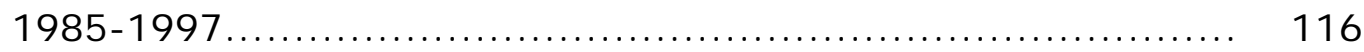

7.2 Überprüfung der Kausalhypothesen.......................... 118

7.2.1 Statistische Vorgehensweise................................ 118

7.2.2 Spezifikation des Analysemodells für das politische Interesse... 121

7.2.2.1 Geschlechtsspezifische Unterschiede bei der Erklärung des Politikinteresses.............................................. 132

7.2.2.2 Interaktionseffekte hinsichtlich der Stabilität des Politikinteresses.................................................. 139

7.2.3 Spezifikation des Analysemodells für die Wertorientierungen.... 143

7.2.3.1 Geschlechtsspezifische Unterschiede bei der Erklärung der Wertorientierungen......................................... 151

7.2.3.2 Interaktionseffekte hinsichtlich der Stabilität der Wertorientierungen............................................ 158

$8 \quad$ Zusammenfassung und Diskussion............................ 160

$9 \quad$ Fazit. 


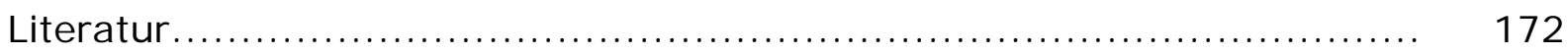




\section{Zusammenfassung}

Die Frage, welche Rolle Sozialisationserfahrungen in Kindheit und Jugend für die Genese der politischen Identität im Erwachsenenalter spielen, gehört zu den zentralen Themen der politischen Sozialisationsforschung. Gegenstand der vorliegenden Studie ist daher die oft formulierte, aber nur selten geprüfte Hypothese, die mittlere Jugendzeit sei eine besonders wichtige und ,formative Phase' für die Ausbildung der individuellen politischen Identität. Im Zentrum der empirschen Analyse stehen drei konkrete Fragen. Zum einen wird geprüft, wie stabil Politikinteresse und Wertorientierungen im Erwachsenenalter zwischen dem 30. und dem 43. Lebensjahr sind. Die zweite Frage untersucht den Einfluss latenter und manifester Sozialisationserfahrungen in der mittleren Adoleszenz auf politisches Interesse und Werte im 30. Lebensjahr. Ferner wird der Frage nachgegangen, welchen Einfluss spätere Lebenserfahrungen auf die genannten Untersuchungsgegenstände haben. Die Grundlage der statistischen Analyse, die u.a. Strukturgleichungsmodelle verwendet, bildet eine dreiwellige, 27 Jahre erfassende Panelstudie ehemaliger Gymnasiasten aus Nordrhein-Westfalen. In der untersuchten Kohorte erweisen sich sowohl das Politikinteresse als auch die Werte im Erwachsenenalter als stabil. Es wird gezeigt, dass bis zur mittleren Adoleszenz wesentliche politische Sozialisationsprozesse stattfinden, die langfristig ,formativ“ wirksam sind. Dabei sind die frühen latenten politischen Sozialisationserfahrungen für die Ausbildung der Werte wichtiger als für die Höhe des Politikinteresses. Die manifesten politischen Sozialisationseinflüsse während der Jugendzeit hingegen haben einen deutlich stärkeren Einfluss auf das Politikinteresse als auf die Wertorientierung. Gleichzeitig wird aber auch sichtbar, dass der Prozess der politischen Sozialisation mit der Jugend nicht abgeschlossen ist. Auch in der Zeit nach der Adoleszenz finden relevante Sozialisationseinflüsse statt. Dies gilt insbesondere für die Entwicklung der Wertorientierungen. Als weiteres wesentliches Ergebnis sind die z.T. gravierenden Geschlechtsunterschiede in der politischen Sozialisation zu nennen. So bestätigt die vorliegende Analyse die Ergebnisse anderer Untersuchungen, wonach Frauen über ein geringeres Politikinteresse verfügen als Männer. Darüber hinaus zeigt sich, dass die frühen Sozialisationseffekte bei Frauen stärker wirken als bei Männern. Zudem ist die Erklärungskraft der Prädiktoren in der Gruppe der Frauen insgesamt höher. Diese Ergebnisse verweisen darauf, dass bei den Geschlechtern im politischen Lernprozess unterschiedliche Mechanismus wirksam sind. 


\section{Abstract}

One of the central subjects of political and social research is the impact of socialization during childhood and adolescence on political identity of the adult. Main issue of the current study is the often made but seldom tested assumption of "impressionable years" during adolescence. First question is the stability of political interest and value orientation during midlife. Second issue is the impact of latent and manifest socialization during adolescence on political interest and attitude in the age of 30 . In addition is investigated, how important later made experiences are in regard of both subjects. The statistical analysis is based on a panel of former grammar school pupils in western Germany which were questioned in three survey periods over 27 years, and uses structural equation models. Both, political interest and value orientation are found stable in the investigated cohort as adults. It is shown that "formative experiences" during childhood and adolescence have longterm effects. Mainly responsible for the creation of value orientation is the early latent socialization whereas manifest socialization during childhood and adolescence affects political interest more than the attitude. However, it is seen that political socialization is a continuous process active also during adulthood and mainly affecting value orientation. In addition, relevant gender differences are found: women are less interested in politics than men, what supports the results of other studies, while early socialization is more relevant in female than male individuals. Furthermore, the predictive value of the independent variables is higher in the female group indicating different mechanisms of political learning. Thus, the idea of "impressive years" in the middle of adolescence with "formative" long-term impact on the political life of adults can be supported. 


\section{Tabellen- und Abbildungsverzeichnis}

Tabelle Titel

Seite

Tabelle 6.1 Zusammensetzung der Untersuchungs- und ALLBUS-

Stichprobe nach sozio-demographischen

Merkmalen

Tabelle 6.2 Verteilung des politischen Interesses und der Wertorientierungen in Untersuchungs- und ALLBUS-

Stichprobe.

100

Tabelle 6.3 Panelausfälle

102

Tabelle 6.4 Vergleich zwischen Panelausfällen und der Untersuchungsstichprobe von $1997 . . . \ldots \ldots \ldots \ldots \ldots \ldots \ldots . \ldots \ldots$

Tabelle 6.5 Variablen zur Sozialisation.............................. 105

Tabelle 7.1 Subjektives politisches Interesse, 1985-1997............ 108

Tabelle 7.2 Wichtigkeit von Politik, 1985-1997..................... 109

Tabelle 7.3 Individuelle Stabilität des subjektiven politischen Inte-

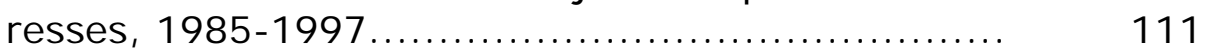

Tabelle 7.4 Individuelle Stabilität der Wichtigkeit von Politik, 1985-

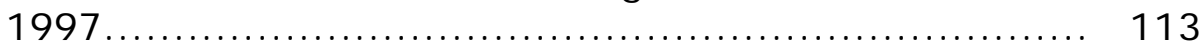

Tabelle 7.5 Postmaterialismus, 1985-1997...................... 115

Tabelle 7.6 Individuelle Stabilität von Postmaterialismus, 19851997.

Tabelle 7.7 Polychorische Korrelationen der Indikatoren mit 'Subjektivem politischen Interesse' basierend auf der Untersuchungsstichprobe von max. 1596

Tabelle 7.8 Standardisierte Faktorladungen der latenten Konstrukte............................................ 124

Tabelle 7.9 Modellanpassung für das Ausgangsmodell............... 126

Tabelle Modellanpassung für das modifizierte Modell, Endmo-

7.10 dell................................................

Tabelle Standardisierte und unstandardisierte Pfadkoeffizienten

7.11 für Politikinteresse, Endmodell.

Tabelle Totale und indirekte Effekte auf das Politikinteresse

7.12 1985: Standardisierte und unstandardisierte Effekte 
Tabelle

7.13

Tabelle

7.14

Tabelle

7.15

Tabelle

7.16

Tabelle

7.17

Tabelle

7.18

Tabelle

7.19

Tabelle

7.20

Tabelle

7.21

Tabelle

7.22

Tabelle

7.23

Tabelle

7.24

Tabelle

7.25

Tabelle

7.26

Tabelle

7.27
Gruppenvergleich zwischen Männern und Frauen, Modellanpassung für das Ausgangs- und Endmodell......

Standardisierte und unstandardisierte Koeffizienten für Gruppenvergleich zwischen Männern und Frauen...

Gruppenvergleich Geschlecht: totale und indirekte unstandardisierte Effekte.

Übersicht über die im Kausalmodell getesteten Hypothesen zum politischen Interesse.

Gruppenvergleich zwischen Befragten mit schwachem und starkem Politikinteresse, Modellanpassung für das Ausgangs- und Endmo-

dell....

Gruppenvergleich zwischen Befragten mit hoher Bildung und niedrigeren Bildung, Modellanpassung für das Ausgangs- und Endmodel.

Polychorische Korrelationen der Indikatoren mit postmaterialistischen Wertorientierungen, Ingelhart- Index basierend auf der Untersuchungsstichprobe von max. 1596

Modellanpassung für das Ausgangsmodell

Modellanpassung für das modifizierte Modell, Endmodell.

Standardisierte und unstandardisierte Pfadkoeffizienten für Wertorientierungen, Endmo-

dell.....

Totale und indirekte Effekte auf Wertorientierungen 1985, standardisierte und unstandardisierte Effekte......

Gruppenvergleich zwischen Männern und Frauen, Modellanpassung für das Ausgangs- und Endmodell......

Standardisierte und unstandardisierte Koeffizienten für Gruppenvergleich zwischen Männern und Frauen.

Gruppenvergleich Geschlecht - totale und indirekte, standardisierte und unstandardisierte Effekte.

Übersicht über die im Kausalmodell getesteten Hypothesen zu Wertorientierungen 
Tabelle 7.28 Gruppenvergleich zwischen Befragten mit schwachem und starkem Politikinteresse, Modellanpassung für das Ausgangs- und Endmo-

dell...

Tabelle 7.29 Gruppenvergleich zwischen Befragten mit hoher Bildung und niedrigeren Bildung, Modellanpassung für das Ausgangs- und Endmo-

dell.

\section{Verzeichnis der Abbildungen}

Nr.

Titel

Seite

7.1 Ausgangsmodell zur Erklärung des politischen Interesses.

7.2 Endmodell zur Erklärung des politischen Interesses.

Endmodell zur Erklärung des politischen Interesses bei Frauen...

Endmodell zur Erklärung des politischen Interesses bei Männern.

Ausgangsmodell zur Erklärung der Wertorientierungen.

Endmodell zur Erklärung der Wertorientierungen

Endmodell zur Erklärung der Wertorientierungen bei Frauen.

Endmodell zur Erklärung der Wertorientierungen bei Männern. 


\section{Verzeichnis ,Fragebogentext und Kodierung“}

Nr.

Titel

Seite

6.1 Variable, Politikinteresse' und ,Wichtigkeit von Politik und öffentlichem Leben'...................... 82

$6.2 \quad$ Variable

,Postmaterialismus'....

6.3

Die Variablen zu den sozio-ökonomischen Res-

sourcen während der Jugendzeit.

6.4

Kognitive Kompetenz in der Ju-

gend.....................

6.5

Latente politische Sozialisation in der Familie.

6.6

Latente politische Sozialisation in der Schule..........

6.7

Manifeste politische Sozialisation in der Schule........

6.8

Politische Partizipation in der Jugendzeit.

6.9

Sekundäre Sozialisationserfahrungen 


\section{$1 \quad$ Einleitung und Fragestellung}

Im Zentrum der vorliegenden Arbeit steht die Frage nach dem Einfluss jugendlicher Sozialisationserfahrungen auf die politische Identität im Erwachsenenalter.

Dass der Jugendphase im Prozess der politischen Identitätsbildung eine besondere Bedeutung zukommt, wird von Sozialwissenschaftlern seit geraumer Zeit angenommen. So vermutete bereits Mannheim vor achtzig Jahren, dass individuelle politische Orientierungen und Verhaltensdispositionen vor allem während einer ,formativen Phase' in der Jugend geprägt werden und im späteren Lebensverlauf weitgehend stabil bleiben bzw. sich zunehmend verhärten (Mannheim 1928). Aber auch neuere Ansätze wie die These vom postmaterialistischen Wertewandel (Inglehart 1971, 1977 und 1989) oder das Konzept der politischen Generationen nach Fogt (1982) basieren auf der Sozialisationsthese und gehen von einer entwicklungspsychologisch begründeten formativen Phase und der anschließenden Persistenz politischer Orientierungen und Verhaltensweisen aus.

Den Sozialisationsthesen gemeinsam ist die Annahme einer besonders sensiblen Phase vor dem Erwachsenenalter, in der politische Informationen verstärkt aufgenommen und eigene politische Orientierungen herausbildet und geprägt werden. Unterschiede zwischen diesen Theorieansätzen bestehen in der jeweiligen Definition des Begriffs. Zum einen unterscheiden sich die Vorstellungen darin, in welchem Ausmaß die formative Phase spätere politischen Orientierungen determiniert. Zum anderen ist umstritten, in welcher zeitlichen Phase solche Prägungen stattfinden (vgl. Kapitel 2.6).

Darüber hinaus differieren die Vorstellungen über die Wirkmechanismen, die dieser formativen Prägung zugrunde liegen. Während Inglehart z.B. die ökonomischen Lebensbedingungen in der formativen Phase des Lebensverlaufes als die entscheidende Determinante im Sozialisationsprozess ansieht, betonen andere Autoren den Einfluss bestimmter Sozialisationsinstanzen für die politische Identitätsentwicklung. Herz (1987) 
etwa hebt die Bedeutung schulischer Sozialisation für die langfristige Prägung von gesellschaftlich dominierenden Werten hervor.

Auch wenn eine allgemein akzeptierte Definition des Begriffs der ,formativen Phase' fehlt, so sind prägende, d.h. langfristig wirksame Einflüsse vor dem Erwachsenenalter eine wesentliche Voraussetzung für das grundsätzliche Konzept. Die folgende empirische Analyse untersucht daher die Frage, welche Bedeutung Erfahrungen aus der Jugendzeit auf zwei wesentliche Aspekte der politischen Identität im Erwachsenenalter haben - auf das Politikinteresse und auf politische Wertorientierungen (s. Kapitel 4).

Eine direkte Überprüfung der These einer frühen Prägung vor dem Erwachsenenalter scheitert in der empirischen Sozialforschung in aller Regel an der mangelhaften Datenlage (vgl. Kapitel 6). Prospektive Panel-Studien, die mehrere Dekaden umfassen und zugleich Informationen über frühe Sozialisationserfahrungen im Elternhaus, in der Schule und anderen wichtigen Sozialisationsinstanzen enthalten, sind zumeist nicht verfügbar.

Mit der vom Kölner Institut für Angewandte Sozialforschung im Jahr 1969 initiierten dreiwelligen Panelstudie ehemaliger Gymnasiasten (Meulemann et al. 1987) liegt ein Datensatz vor, der eine Untersuchung politischer Sozialisationserfahrungen aus der Zeit der mittleren Adoleszenz ${ }^{1}$ und ihrer Einflüsse auf zwei Aspekte der politische Identität (,Politikinteresse' und ,politische Wertorientierungen', vgl. ausführlich Kapitel 6) im Erwachsenenalter erlaubt. Es handelt sich hierbei um eine Stichprobe ehemaliger Gymnasiasten, die 1969/70 im Alter von durchschnittlich 15 Jahren erstmals befragt wurden (Meulemann et al. 1987). Diese Gymnasiastenbefragung und die zeitgleich durchgeführte Elternerhebung enthielten unter anderem Fragen zur sozialen Herkunft und zu Sozialisationserfahrungen der Jugendlichen. In den Jahren 1984/85 und 1997 fanden zwei Wiederbefragungen der inzwischen etwa 30 bzw. 43 Jahre alten früheren Schüler statt. Die beiden abhängigen Variablen bzw. Konstrukte

\footnotetext{
1 Die Sozialisationserfahrungen der mittleren Adoleszenz werden im folgenden ,frühe Sozialisationserfahrungen' genannt. Die daran anschließenden Erfahrungen werden ,späte' oder ,sekundäre' Sozialisationserfahrungen genannt.
} 
,Politikinteresse' und ,Wertorientierungen' wurden in diesen beiden Wiederbefragungen erfragt.

Trifft die These einer frühen Prägung zu, dann sollten sich politisches Interesse und politische Wertorientierungen auf der Individualebene als relativ stabil erweisen. Nur wenn die empirische Analyse zeigt, dass beide Untersuchungsgegenstände tatsächlich ein Mindestmaß an Stabilität aufweisen, kann eine ,Prägung' im Sinne einer ,formativen Phase' ursächlich sein. Anschließend kann dann geprüft werden, ob Erfahrungen aus der Jugendzeit Einfluss auf Politikinteresse und Wertorientierungen im 30. Lebensjahr haben.

Wie im Theorieteil dargelegt wird, spielen sogenannte latente ${ }^{2}$ Sozialisationserfahrungen eine nicht unerhebliche Rolle im politischen Orientierungsprozess der Jugendlichen (vgl. Kapitel 2). Ob diese Einflüsse allerdings eine langfristige Wirkung auf die Entwicklung von politischem Interesse und Wertorientierungen ausüben, ist bislang nicht bekannt. Aus diesem Grund wird in der vorliegenden Untersuchung besonderes Augenmerk auf die langfristigen Auswirkungen latenter Sozialisationserfahrungen in Familie und Schule gelegt. Konkret wird danach gefragt, welchen Einfluss elterliche Erziehungsvorstellungen und Aspekte des Familienklimas auf politisches Interesse und Wertorientierungen im 30. Lebensjahr haben. Im Hinblick auf schulische Sozialisationserfahrungen wird untersucht, welche Bedeutung Auseinandersetzungen mit Autoritäten in der Schule für die betreffenden politischen Merkmale haben.

Es ist zu vermuten, dass neben den beschriebenen latenten Sozialisationseinflüssen auch weitere Faktoren aus der jugendlichen Sozialisationsphase langfristig wirksam sind. Dazu gehören hauptsächlich sogenannte manifeste ${ }^{3}$

\footnotetext{
2 Latenter politischer Sozialisation meint jenes Lernen, das nicht originär politisch ist, aber indirekt auf politische Orientierungen und Verhaltensweisen wirkt, siehe dazu ausführlich Kapitel 2.

3 Unter manifester politische Sozialisation wird dagegen direktes politisches Lernen verstanden, siehe dazu ausführlich Kapitel 2.
} 
politische Erfahrungen (vgl. Kapitel 2), aber auch individuelle Ressourcen wie etwa die soziale Herkunft.

Darüber hinaus ist aus theoretischer Sicht durchaus plausibel, dass biographische Erfahrungen im späteren Lebensalter das Interesse an politischen Angelegenheiten bzw. politische Wertorientierungen beeinflussen können (vgl. Kapitel 4). Aus diesem Grunde werden zusätzlich auch spätere biographische Erfahrungen aus der Zeit zwischen dem 15. und 30. Lebensjahr in der Untersuchung berücksichtigt.

Für die anschließende Untersuchung ergeben sich drei konkrete Fragestellungen:

1. Wie stabil sind politisches Interesse und politische Wertorientierungen im Erwachsenenalter zwischen dem 30. und dem 43. Lebensjahr?

2. Welchen Einfluss haben latente und manifeste Sozialisationserfahrungen in der mittleren Adoleszenz auf politisches Interesse und politische Wertorientierungen im 30. Lebensjahr?

3. Welchen Einfluss haben spätere Lebenserfahrungen nach der vermuteten ,sensiblen' Phase auf politisches Interesse und politische Wertorientierungen im 30. Lebensjahr?

Auch wenn eine weitgehende Stabilität von Politikinteresse und Wertorientierungen vorliegt, so ist dennoch möglich, dass diesbezüglich in Subgruppen Unterschiede bestehen - und somit Interaktionseffekte vorliegen. Daraus folgt eine vierte Fragestellung:

4. Gibt es Faktoren, welche die Stabilität des politischen Interesses und der politischen Werte beeinflussen? 


\section{Theoretischer Hintergrund der politi- schen Sozialisation}

\subsection{Begriff und Gegenstand der politischen Sozia- lisation - Politische Sozialisation als Lernpro- zess}

Der Begriff der ,politischen Sozialisation' wurde Ende der 50er Jahre von Easton (1957) und Hyman (1959) in die amerikanische Politikwissenschaft eingeführt (Hopf 1997: 11, Greiffenhagen 2002: 408). Die mittlerweile sehr zahlreich vorliegenden Begriffsbestimmungen politischer Sozialisation unterscheiden sich nach Greiffenhagen sowohl im Verständnis von ,politisch' als auch in der Definitionen von ,Sozialisation'. In der Summe lassen sich die verschiedenen Auffassungen zu zwei allgemein akzeptierten Definitionen zusammenfassen. Politische Sozialisation kann demnach entweder definiert werden als „den Lernprozess, innerhalb dessen ein Individuum sich jene Persönlichkeitsmerkmale, Kenntnisse, Fähigkeiten und Werte aneignet, welche politisches Bewusstsein und politisches Verhalten strukturieren und lenken, also politisch relevant sind; oder als den Lernprozess, innerhalb dessen einem Individuum jene Persönlichkeitsmerkmale vermittelt werden“ (Greiffenhagen 2002: 408).

Bei der politischen Sozialisation handelt es sich folglich um einen Entwicklungsprozess, an dem verschiedene gesellschaftliche Ebenen beteiligt sind (Claußen 1982): auf der Mikroebene vollzieht sich der individuelle Lernprozess, in dessen Verlauf eine Person politische Orientierungen und Verhaltensmuster erwirbt, eine politische Identität ausbildet ${ }^{4}$. Diese Lernvorgänge betreffen sowohl kognitive, affektiv-motivationale als auch behaviorale Aspekte der politischen Identität (s.u.).

Die Orte, in denen die individuellen Lernprozesse in Auseinandersetzung mit der jeweiligen sozialen Umwelt stattfinden, die sog. Sozialisationsinstanzen

\footnotetext{
${ }^{4}$ Die Begriffe individuelle politische Sozialisation, politische Identitätsbildung und -entwicklung werden im Folgenden synonym verwendet.
} 
(vgl. Kapitel 3), lassen sich dagegen auf der Mesoebene ansieden. Zu den zentralen politischen Sozialisationsinstanzen zählen dabei jene Lebensräume, in denen sich Menschen dauerhaft oder längere Zeit aufhalten bzw. denen sie ausgesetzt sind. In der Literatur werden hauptsächlich Familie (oder familienähnliche Lebensgemeinschaften), Schule (bzw. vorschulische Institutionen und Hochschulen), Gleichaltrigengruppe, Medien und Arbeitsplatz genannt (vgl. Claußen 1996: 31f., Hopf/Hopf 1997). Aber auch das politische Lernen in der Bundeswehr oder beim Zivildienst, in Verbänden und Organisationen oder auch in der Freizeitwelt wird in der Literatur erwähnt und ist Gegenstand von empirischen Untersuchungen (vgl. Claußen/Geißler 1996).

Der äußere Bezugsrahmen der politischen Sozialisation schließlich bildet die politische Gemeinschaft, das politische System eines Staates (Makroebene). Aus der Makroperspektive betrachtet kann daher für demokratische Staaten der Prozess der politischen Sozialisation als ,Hineinwachsen' in die politische Kultur des Landes beschrieben werden, welcher zum Ziel hat, das Individuum zu einem gleichberechtigten und politisch handlungsfähigen Mitglied der politischen Gemeinschaft zu machen (vgl. Almond/Verba 1980: 9, Kulke 1982: 742).

\subsection{Die Bedeutung der politischen Sozialisation für die Demokratie}

Welche Bedeutung der politischen Sozialisation für die Stabilität eines politischen Systems zugeschrieben wird, zeigt sich besonders im Konzept der politischen Unterstützung des Systemtheoretikers Easton (Easton 1975, Easton/Dennis 1969).

Für Easton hängt die Stabilität demokratischer Systeme maßgeblich von der Akzeptanz und Unterstützung ihrer Bürger ab. Er unterscheidet zwischen spezifischen und diffusen Unterstützungsformen: spezifische Unterstützung liegt dann vor, wenn die konkreten Leistungen des politischen Systems, der Institutionen und seiner Akteure den an sie gestellten Forderungen entsprechen. Werden Diskrepanzen zwischen Anforderungen und Leistungen des Staates wahrgenommen, so kann die spezifische Unterstützung relativ schnell absin- 
ken, etwa bei Kürzungen sozialer Leistungen oder Anstieg der Arbeitslosenquote.

Die diffuse Unterstützung bezieht sich dagegen nicht auf konkrete Leistungen des Staates sondern auf allgemeine grundsätzliche Eigenschaften wie Vertrauenswürdigkeit oder Gemeinwohlorientierung des politischen Systems und seiner Objekte (Fuchs 1989). Die diffuse Unterstützung ist stärker im individuellen Überzeugungssystem verankert und folglich auch geringeren Schwankungen ausgesetzt als die spezifische Unterstützung.

Während ein (kurzfristiger) Mangel an spezifischer Unterstützung nicht grundsätzlich zum Verlust der Legitimität und damit zur Instabilität eines politischen Systems führt, ist die diffuse Unterstützung zentral für die Stabilität demokratischer Staaten. Ein politisches System kann auf Dauer nur dann bestehen, wenn die zentralen politischen Werte, Normen und Machtstrukturen grundsätzlich Zustimmung und Sympathie in der Gesellschaft finden und die Bürger sich mit der bestehenden politischen Ordnung identifizieren.

Die Schaffung diffuser politischen Unterstützung stellt für Easton daher das wesentliche Ziel der politischen Sozialisation dar (Easton 1975).

Neben der diffusen Unterstützung politischer Ordnungsprinzipien ist auch ein bestimmtes Maß an politischem Interesse und Partizipation der Bürger eine wichtige und notwendige Voraussetzung für die Funktionsfähigkeit demokratischer Systeme. Oder wie Verba et al. formulierten: "Citizen participation is a heart of democracy“ (Verba et al 1995: 1, vgl. auch van Deth 2000). Eine in diesem Sinne ,erfolgreiche' politische Sozialisation ist daher für demokratische Staaten von großer Bedeutung. 


\subsection{Politische I dentitätsbildung als Entwicklungs- aufgabe des J ugendalters}

Für den Entwicklungspsychologen Helmut Fend ist der ,mündige Bürger' das Idealbild einer gelungenen politischen Sozialisation. Als Merkmal des ,mündigen Bürgers' formuliert er drei wesentliche Entwicklungsziele der politischen Sozialisation, auf die eine demokratische Ordnung angewiesen ist (Fend 1991: 137):

1. Zum einen sind Akzeptanz der Verfassungsziele und Internalisierung demokratischer Normen und Spielregeln erforderlich (vgl. Konzept der diffusen Unterstützung).

2. Neben allgemeiner politischer Loyalität sind ,kenntnisreiche Urteilsfähigkeit' und ,gesundes Misstrauen' gegenüber den politischen Vorgängen erwünscht, um Bedrohungen der demokratischen Ordnung erkennen und Machtkontrolle sicherstellen zu können.

3. Die dritte Anforderung, die der mündige Bürger zu erfüllen hat, ist die Entwicklung politischer Partizipationsbereitschaft bzw. -fähigkeit. Die Bereitschaft und Fähigkeit, sich am politischen Geschehen zu beteiligen, ist der Kern der Demokratie und lebensnotwendig für demokratische Systeme (siehe oben, Hoffmann-Lange 1995: 14). Neben der klassischen Beteiligungsform des politische Wählens gehören hierzu auch umfassendere und unkonventionelle Bürgerbeteiligungen wie das Engagement in Bürgerinitiativen, Unterschriftenaktionen oder die Teilnahme an Demonstrationen zur politischen Handlungsbereitschaft.

Für Fend stellt sich die politische Identitätsbildung, die Entwicklung zum ,mündigen Bürger' als eine Entwicklungsaufgabe der Jugendzeit dar.

Die Schwierigkeit, vor der die Heranwachsenden dabei stehen, ist im Spannungsverhältnis zwischen den verschiedenen Anforderungen zu sehen: Einerseits soll der politisch ,mündige Bürger' willens und in der Lage sein, die politischen Vorgänge aufmerksam und kritisch zu verfolgen. Andererseits soll er zugleich Vertrauen und Loyalität gegenüber der politischen Ordnung aufbringen. Im Spannungsbereich dieser Anforderungen müssen sich Jugendliche 
selbständig bewegen und einen eigenen, unabhängigen Standpunkt finden, der sowohl Loyalität als auch Kritikfähigkeit umfasst (Fend 1991: 137). Außerdem erfordert eine derartige Integrationsleistung, die Heranwachsende im Laufe ihrer politischen Sozialisation erbringen sollen, ein erhebliches Maß an Abstraktions- und Reflexionsvermögen.

\subsection{Dimensionen der politischen I dentitätsbildung}

Die Entwicklung der politischen Identität lässt sich nach Fend (1991: 152f.) in drei Dimensionen unterteilen:

1. die affektiv-motivationale Dimension

2. die kognitive Dimension

3. behaviorale Dimension ${ }^{5}$

\subsubsection{Die affektiv-motivationale Dimension}

Die affektiv-motivationale Dimension umfasst den gesamten Bereich der Bewertung politischer Inhalte sowie die Identifikation mit dem politischen System, mit politischen Institutionen, Gruppierungen und Personen. Auch Gefühle der politischen (internen wie externen) Effektivität und individuelles politisches Interesse fallen nach diesem Konzept in den Bereich der affektivmotivationalen politischen Identitätsbildung.

Vielen empirischen Untersuchungen zufolge werden die affektivmotivationalen Orientierungen schon in früher Kindheit und Jugend entwickelt (van Deth 2005: 4, Barton/Schäfer 2005, Fend 2006: 391, vgl. auch Kap 2.6). Bereits die klassischen Studien der politischen Sozialisationsforschung konnten zeigen, dass schon Kinder und Jugendliche über affektive Bindungen

\footnotetext{
${ }^{5}$ Allerdings zeigt sich, dass die einzelnen Dimensionen sich nicht immer trennen lassen und z.T. auch deutlich miteinander korrelieren.
} 
an politische Symbole, wichtige politische Persönlichkeiten und das politische System verfügen (Easton/Dennis 1969, Hess/Torney 1970).

\subsubsection{Die kognitive Dimension}

Unter dem Begriff der kognitiven politischen Dimension werden politisches Wissen und politische Analysefähigkeit zusammengefasst (Fend 1991: 157). Dazu zählen Kenntnisse über politische Sachverhalte und Ereignisse, politisch bedeutsame Personen und Institutionen sowie intellektuell-analytische Aspekte wie Verständnis für politische Zusammenhänge und die Herausbildung politischer Analyse- und Urteilsfähigkeit.

Die Voraussetzung für den Erwerb dieser kognitiven politischen Fähigkeiten besteht in der Entwicklung von abstraktem Denken und Verständnis für komplexe Zusammenhänge: Nach Erkenntnissen der Entwicklungspsychologie entstehen diese Fähigkeiten etwa ab dem 12 Lebensjahr. Dementsprechend zeigen empirische Befunde, dass mit zunehmenden Alter der Jugendlichen auch die kognitiven politischen Fähigkeiten an steigen (Fend 2006: 113f).

\subsubsection{Die behaviorale Dimension}

Die behaviorale Dimension umfasst sowohl politische Partizipationsbereitschaft als auch tatsächliche Beteiligung am politischen Prozess. Die politische Partizipationsforschung unterscheidet zwischen klassisch-konventionellen Beteiligungsformen wie Wahlbeteiligung oder Parteimitgliedschaft und unkonventionellen Partizipationsmöglichkeiten wie der Teilnahme an einer Demonstration oder einem Boykott. Die unkonventionellen Formen lassen sich weiter unterscheiden in legale Formen der politischen Partizipation wie Demonstrationen oder Unterschriftensammlung und in illegale Formen wie Hausbesetzung, Verkehrsblockaden oder gewalttätige Angriffe auf Polizisten (vgl. Uhlinger 1988, Kaase 1987).

Zusätzlich zu den originär politischen Beteiligungsformen zählen zu der behavioralen Dimension auch Tätigkeiten aus dem ehrenamtlichen sozialen Bereich oder Formen der Mitbestimmung (z.B. in der Schule und im Beruf). 


\subsection{Latente und manifeste politische Sozialisation}

Die Unterscheidung zwischen latenter und manifester politischer Sozialisation geht auf den Politikwissenschaftler Greenstein zurück (Greenstein 1965, vgl. auch Hopf/Hopf 1997, Wasmund 1982a: 40). Da bei der Erforschung von politischer Sozialisation auch a priori nicht politische Erfahrungen mit einzubeziehen seien, führte Greenstein das Begriffspaar ,latenter' und ,manifester' Sozialisation in die politische Sozialisationsforschung ein.

Unter latenter politischer Sozialisation ist demzufolge jenes Lernen zu verstehen, das nicht originär politisch ist, aber auf politische Orientierungen und Verhaltensweisen wirkt. Die politischen Dimensionen der Persönlichkeit, werden also ,auf dem Umweg' über unpolitische Persönlichkeitsmerkmale mit beeinflusst (Geißler 1996: 61) ${ }^{6}$. Manifeste politische Sozialisation meint hingegen unmittelbar politisches Lernen (s.u.).

Die latente politische Sozialisation wird in der Literatur als zweistufiger Prozess aufgefasst, in dem sich zunächst allgemeine Persönlichkeitseigenschaften und Dispositionen herausbilden, die dann später politische Vorstellungen und politisches Handeln beeinflussen (vgl. Wasmund 1982a). Bei latenten Sozialisationsprozessen kommen der Familie bzw. familienähnlichen Strukturen als zentralem Ort für frühe Lernerfahrungen und Persönlichkeitsentwicklung eine erhebliche Bedeutung zu (vgl. Geißler 1996: 62). Bedeutsame Faktoren des latenten politischen Lernens sind dabei insbesondere innerfamiliäre Strukturen wie elterliches Erziehungsverhalten, Autoritätsverhältnisse, Kommunikationsstile, Familienklima oder Persönlichkeitsmerkmale der Eltern.

Aber auch Einflüsse von Kindergarten- und Schulalltag und Peer-Beziehungen wirken sich auf die (politische) Persönlichkeitsentwicklung aus. So werden etwa im Schulkontext dem Schul- und Klassenklima, den Mitbestimmungs-

\footnotetext{
${ }^{6}$ Die Idee der latenten politischen Sozialisation ist allerdings sehr viel älter. Beispielsweise beschrieb die Forschergruppe um Adorno und Horkheimer in ihrer These der ,autoritären Persönlichkeit' latente Sozialisationsprozesse (Adorno et al. 1950). Dieser liegt die Annahme von langfristig wirksamen frühkindlichen Prägungen zugrunde, der zufolge die Autoritätsverhältnisse, die man in der Kindheit in der Familie erlebt, entscheidend dafür sind, wie man im späteren Lebensverlauf auf staatliche und gesellschaftliche Autoritäten reagiert.
} 
möglichkeiten im Unterricht oder den Lehrer-Schüler-Beziehungen latente Sozialisationswirkungen zugeschrieben (Kandzora 1996).

Mit Weissberg (1974: 20) lassen sich die unterschiedlichen Merkmale der latenten politischen Sozialisation in vier Gruppen einordnen: (1) Persönlichkeitseigenschaften, (2) individuelle Fähigkeiten, (3) soziale Identifikationen und (4) soziale Rollen.

Zu den (1) Persönlichkeitsmerkmalen, die politisches Denken und Handeln beeinflussen, zählen z.B. Autoritarismus, Feindseligkeit, Entfremdung, Soziabilität, Extraversion oder auch Selbstwirksamkeitsüberzeugung, die zu einer entsprechenden politischen Wirksamkeitsüberzeugung (,Political Efficacy', vgl. Vetter 1997) führen kann. Die (2) individuellen Fähigkeiten, die in diesem Kontext bedeutsam sein können, sind Fertigkeiten wie abstraktes Denken, rhetorisches Talent oder soziale Kompetenz. Dem Bereich der (3) sozialen Identifikationen, die politische Orientierungen und Verhaltensweisen beeinflussen, sind z. B. subjektive Schichtzugehörigkeit, ethnische Herkunft oder religiöse Bindung zuzuordnen. Dass derartige Identifikationen etwa bei politischen Wahlentscheidungen wirksam werden, ist bereits seit der Frühzeit der empirischen Wahlforschung bekannt (Lazarsfeld 1944, Lipset/Rokkan 1967). $\mathrm{Zu}$ den politisch einstellungs- und verhaltenswirksamen (4) sozialen Rollen und Rollenerwartungen zählen gelernte Geschlechterrollen oder Rollenverhältnisse in der Familie und Schule.

Nach Dawson et al. (1977: 99f.) lassen sich drei Formen des latenten politischen Lernens unterscheiden: die interpersonelle Übertragung, das Erfahrungslernen und Generalisierungen.

Das Modell der interpersonalen Übertragung geht davon aus, dass die frühen Erfahrungen, die Kinder mit Autoritätspersonen - insbesondere den Eltern machen, später auf politische Autoritäten übertragen werden. Werden also die Eltern z.B. als zuverlässig und wohlwollend wahrgenommen, so werden politische Autoritäten auch eher als vertrauenswürdig angesehen.

Der Idee des Erfahrungslernens liegt die Vorstellung zugrunde, dass Aktivitäten und Erfahrungen in Alltagssituationen Modelle für späteres politisches Handeln darstellen. Fähigkeiten und Erkenntnisse, die in im Familienkontext, bei Gleichaltrigen oder in der Schule gewonnen werden, können später der 
Orientierung im politischen Raum zugute kommen. Zu solchen Erfahrungen gehören beispielsweise Mitsprachemöglichkeiten der Kinder bei Familienoder Schulentscheidungen oder Übung in Konfliktlösungsstrategien (vgl. auch Almond/ Verba 1965). Die dritte Form des Lernens, die Generalisierung, geht vom Einfluss allgemeiner grundsätzlicher Überzeugungen auf die Betrachtungsweise von politischen Sachverhalten aus. Naive Grundeinstellungen den Mitmenschen gegenüber kann z.B. auf den Bereich der Politik übertragen werden und zu politischer Naivität führen.

Manifeste politische Sozialisation beinhaltet sowohl intendierte als auch nicht intendierte politische Lernprozesse, die prinzipiell in allen Sozialisationskontexten stattfinden können. Intendierte Sozialisationsprozesse liegen dann vor, wenn versucht wird, den Sozialisanden gezielt zu informieren bzw. zu beeinflussen. Ein klassisches Beispiel für intendierte manifeste politische Sozialisation ist etwa der Politik- oder Sozialkundeunterricht in der Schule, in dem es explizit um die Vermittlung politischen Wissens geht. Aber auch politische Gespräche zwischen Eltern und Kindern, in denen die Eltern Bewertungen und Kommentare zu politischen Personen oder Ereignissen abgeben, zählen zur intendierten manifesten Sozialisation. Verfolgt ein Jugendlicher ein politisches Gespräch hingegen nur beiläufig, so wird dies als nicht- intendierte manifeste politische Sozialisation bezeichnet (vgl. Dawson et al. 1977). 


\subsection{Modelle und Theorien der Politischen Soziali- sation}

Seit sich die Sozialwissenschaften mit dem Gegenstand der politischen Sozialisation beschäftigen, wurde eine Vielzahl theoretischer Modelle und Ansätze entwickelt, denen - je nach Disziplin und/oder wissenschaftlicher Schule unterschiedliche psychologische und/oder soziologische Grundannahmen sowie verschiedene Demokratieverständnisse zugrunde liegen (vgl. Greiffenhagen 2002: 410f.). Dabei handelt es nach Greiffenhagen zumeist um Ansätze aus den 70er Jahren und um „Einzelbetrachtungen aus eher fachspezifischer Sicht; ein Mangel an integrativer Theorie lässt sich, bis auf wenige Ausnahmen, nicht übersehen“ (Greiffenhagen 2002: 410).

Für dieses Theoriedefizit sind nach Greiffenhagen verschiedene Gründe verantwortlich. Als Hauptursachen sieht sie die starke Interdisziplinarität, die allgemeine Theoriemüdigkeit der jüngeren Sozialwissenschaften, Zeitgeisteffekte sowie die Enttäuschung über die dürftigen Resultate der Forschung an (Greiffenhagen 2002: 215).

In der Literatur gibt es etliche Versuche, die verschiedenen theoretischen Ansätze zur politischen Sozialisation inhaltlich zu ordnen (vgl. etwa Hess/Torney 1970, Greifenhagen 2002, vgl. auch Weiß 1981). Ein Versuch, die verschiedenen Theorieansätze zu systematisieren, unternimmt Kuhlke (1982) in einem Überblicksartikel. Demnach können vier Konzeptkategorien unterschieden werden:

1. Identifikations- und Legitimationskonzepte

2. Interaktions- und Identitätskonzepte

3. Kognitions- und Kooperationskonzepte

4. Konzepte der kritischen Aufklärung, Partizipation und politischgesellschaftlichen Emanzipation

Allerdings gibt die Autorin selbst zu bedenken, dass in vielen Fällen Überschneidungen vorliegen und eine eindeutige Abgrenzung oft nicht gelingt (Kuhlke 1982: 752). 
Zu 1: Die Identifikations- und Legitimationskonzepte verstehen politische Sozialisation als einen Prozess der Übertragung bzw. Übernahme politischer Orientierungen. Zu diesen Konzepten gehören - neben systemfunktionalistischen Theorieansätzen (vgl. Easton/Dennis 1969, siehe auch ausführlicher weiter unten) - auch psychoanalytisch orientierte Ansätze (z.B. Greenstein 1965). Letztere legen den Fokus der Sozialisation auf die frühe Kindheit und gehen davon aus, dass frühzeitig erworbene Orientierungen mehr affektiver Natur sind und oft stattfinden, bevor die kognitiven Voraussetzungen zur Erfassung politischer Inhalte überhaupt gegeben sind. Die früh gelernten politischen Orientierungen und Identifikationen sind diesen Konzepten zufolge wenig veränderbar und strukturieren das späterer politische Lernen vor (Greenstein 1965, siehe auch das ,Primacy Principle'- bzw. Kristallisationsmodell, weiter unten; oder auch das Konzept der Parteiidentifikation nach Campbell et al. 1960).

Zu 2: Den Interaktions- und Identitätskonzepten liegt die Vorstellung zugrunde, dass politische Identität sich durch interaktive Lernprozesse entwickelt. Demzufolge werden politische Lernvorgänge durch Rollenwandel oder Veränderungen der Lebenssituation initiiert. Während für die Identifikationsund Legitimierungskonzepte eine wesentliche Funktion der politischen Sozialisation in der Stabilisierung des sozialen und politischen Systems besteht, liegt nach den Interaktions- und Identitätskonzepten das vornehmliche Ziel von Sozialisationsprozessen im Erwerb eines flexiblen Rollenlernens und handelns zur Ausbildung eines ,Political Self'.

Zu 3: Die Kognitions- und Kooperationskonzepte betrachten den Prozess der politischen Sozialisation aus entwicklungspsychologischer Perspektive. Diese Ansätze gehen von einer altersabhängigen Entwicklung zunehmender kognitiver und sozialer Kompetenzen aus, die in Auseinandersetzung mit der sozialen Umwelt verläuft. Prominente Vertreter solcher entwicklungspsychologischer Konzepte sind vor allem Piaget (1954/73) und Kohlberg (1976). Piagets Modell der Intelligenzentwicklung geht von einem stufenförmigen Entwicklungsprozess kognitiver Strukturen, die von vor-operationalen-konkreten zu abstrakt-operationalen Stadien verläuft (vgl. die Stufen der Moralentwicklung bei Kohlberg 1976). Die Ausbildung politischer Orientierungen ist hier eng 
verknüpft mit kognitiven Voraussetzungen wie der Fähigkeit zu hypothetischem Denken, zu Kausalanalysen oder zur Perspektivübernahme. Eine frühkindliche Prägung wie im Kristallisationsmodell findet nach diesen Konzepten nicht statt. Vielmehr ist hier die Adoleszenz, in der zentrale kognitive und moralische Entwicklungen stattfinden, eine besonders wichtige Phase.

Zu 4: Im Rahmen der Konzepte der kritischen Aufklärung, Partizipation und politisch-gesellschaftlichen Emanzipation vollziehen sich politische Lernprozesse in Abhängigkeit bestehender Gesellschaftsstrukturen. Nach Kuhlke lassen sich zwei Hauptstränge der in dieser Tradition stehenden Untersuchungsanlagen unterscheiden (Kuhlke 1982: 761f.): Zum einen die sozialpsychologisch-psychoanalytischen und die gesellschaftskritisch ausgerichteten Analysen. Hierzu gehören unter anderem die Arbeiten der Autoritarismusforschung der Frankfurter Schule um Adorno (vgl. Adorno 1950). Der zweite Hauptstrang umfasst "sozioökonomische und gesellschaftstheoretisch fundierten Untersuchungen über materielle Bedingungen und politisches Bewusstsein von Arbeitern“ (Kuhlke 1982: 763).

Zu den zentralen Aspekten der politischen Sozialisationsforschung gehört die Bedeutung der verschiedenen Lebensphasen für das politische Lernen (Geißler 1996: 53, Wasmund 1982a: 28). Dabei geht es um die Frage, ob es bestimmte Altersphasen gibt, in denen sich relevante Merkmale der politischen Persönlichkeit entwickeln bzw. politische Inhalte gelernt werden (vgl. die oben beschriebenen entwicklungspsychologischen Konzepte). Und für den Fall, dass solche Phasen existieren, welche politischen Orientierungen und Eigenschaften wann entstehen, und ob diese im weiteren Lebensverlauf stabil bleiben.

Nach Weissberg (1974) lassen sich drei miteinander konkurrierende Modelle des politischen Lernens unterschieden, die jeweils verschiedene Perioden im Lebenszyklus hervorheben:

1. ,Primacy Principle'-Modell (Kristallisationsthese)

2. ,Intermediate Period'-Modell

3. ,Recency'-Modell 


\subsubsection{Das ,Primacy Principle'-Modell}

Das in psychoanalytischer Tradition stehende ,Primacy Principle'-Modell geht von der Vorstellung aus, dass die Sozialisationseinflüsse der frühen Kindheit besonders bedeutsam für die politische Identitätsbildung (z.B. Easton/Dennis 1969, Hess/Torney 1970) sind. Demnach werden bereits im frühen Kindesalter Bausteine einer politischen Grundorientierung erworben. Diese politische Grundorientierung bildet die Basis für die spätere politische Identität einer Person. Die besondere Bedeutung früh entstandener Orientierungen erklärt sich aus ihrer Stabilität: Der ,Primacy Principle'-These zufolge, entstehen in der frühen Kindheit grundlegende Persönlichkeitsmerkmale und Orientierungen (Primat der frühkindlichen Sozialisation), die weniger veränderbar sind als später entstandene. Den früh erworbenen Kenntnissen und Orientierungen wird daher ein starker Einfluss auf die politischen Einstellungen und Verhaltensweisen im Erwachsenenalter zugeschrieben.

Die Persistenz der frühen Prägungen wird damit erklärt, dass die Lernprozesse in einer besonders responsiven und unkritischen Phase der menschlichen Entwicklung stattfinden (vgl. Pawelka 1977: 94). Das politische Lernen in der frühen Kindheit ist grundsätzlich stark affektiv gefärbt und besteht zumeist, da die kognitive Fähigkeit zur Kritikfähigkeit in diesem Alter noch nicht gegeben ist, in der unkritischen Übernahme politischer Orientierungen naher Bezugspersonen (vgl. Greenstein 1965). Zu den in früher Kindheit entwickelten Orientierungen gehören insbesondere grundlegende politische Loyalitäten, Identifikationen mit dem politischen System, seinen Institutionen und Autoritäten sowie Vorstellungen über soziale Gruppen und Feindbilder (vgl. Wasmund 1982a: 28).

Nach dieser Phase gilt die politische Persönlichkeit zwar noch nicht als endgültig ausgereift, starke Veränderungen der politischen Grundorientierungen sind allerdings eher unwahrscheinlich: Neue Sozialisationserfahrungen ergänzen die bestehenden Grundorientierungen eher, als dass sie diese grundsätzlich revidieren (Greenstein 1965: 79, vgl. ,Dissonanztheorie‘ nach Festinger 1957). Dieser Prozess lässt sich dadurch erklären, dass die einmal entstandenen Eigenschaften und Orientierungen den Erwerb neuer spezifischeren 
,Traits' strukturieren (Strukturierungsprinzip) und als Filter für zukünftige politische Lernprozesse wirken.

Als wichtigste Sozialisationsinstanz der frühen Kindheit (vgl. Kapitel 3) kommt dem Modell des frühen politischen Lernens der Familie die größte Bedeutung bei der Genese der politischen Persönlichkeit zu. Die politischen Lernprozesse finden dabei vornehmlich über latente, weniger über manifeste Sozialisationserfahrungen statt (s.o.).

In diesem Lernprozess entfalten Familieneinflüsse eine zweifache Wirkung: Zum einen beeinflusst die Familie durch die Transmission politischer Einstellungen und Werte die individuelle Entwicklung der heranwachsenden Kinder. Gleichzeitig wird durch die Übertragung der politischen Orientierungen auf die nachwachsenden Generationen politische Kontinuität auf der Makroebene hergestellt (,Familie als Transmissionsriemen', vgl. Easton/Dennis 1969).

\subsubsection{Das ,I ntermediate Period'-Modell}

Das ,I ntermediate Period'-Modell hingegen geht davon aus, dass die meisten dauerhaften politischen Orientierungen und Verhaltensweisen erst in der späten Kindheit- und Jugendphase entstehen. Es argumentiert in erster Linie entwicklungspsychologisch: Während in der frühen Kindheit politische Gegenstände und Konzepte noch sehr diffus und unkritisch wahrgenommen und verarbeitet werden, entwickelt sich in Folge der fortgesetzten primären Sozialisation ein differenzierteres Bild vom Gegenstand der Politik (vgl. Weissberg/Jakob 1970, Adelson 1980).

Nach den Erkenntnissen der Entwicklungspsychologie entstehen die kognitiven Voraussetzungen für abstraktes, hypothetisches und logisches Denkvermögen etwa in den Jahren der frühen bis mittleren Adoleszenz, also im Alter zwischen 12 und 16 Jahren (vgl. Adelson 1980: 272, Fend 2006: 113f.). Adelson (1980) kommt in einer Untersuchung zu den politischen Vorstellungen frühadoleszenter Jugendlicher zu dem Schluss, dass diese Entwicklungsjahre entscheidend für das Entstehen politischen Denkens sind und die kognitiven (politischen) Fähigkeiten in dieser Zeit einen Sprung vollziehen. Demnach beginnt im zwölften, dreizehnten Lebensjahr eine tiefgreifende Verände- 
rung im Stil des politischen Denkens. Erst ab diesem Alter sind die Heranwachsenden in der Lage, selbständig politische Sachverhalte logisch zu durchdenken und eigene Standpunkte zu entwickeln ${ }^{7}$. Die Folge ist bei den Jugendlichen eine Abnahme autoritärer Betrachtungsweisen und es entsteht -ansatzweise- die Fähigkeit zur Ideologieentwicklung (Wasmund 1982c: 147).

Auch Fend (1991: 146) geht davon aus, dass die Zeit der frühen Adoleszenz besonders wichtig ist für die Entwicklungen der Persönlichkeit und der kognitiven Fähigkeiten. Seinen Untersuchungen zufolge steigert sich in diesem Alter das politische Begriffsverständnis, das Abstraktionsniveau und die Verknüpfungsfähigkeit; außerdem findet eine Veränderung vom personenbezogenen politischen Denken zu einem institutionsbezogenen statt (Fend 1991: 150).

Allerdings ist das logisch-formale Denken nicht die alleinige Voraussetzung für politisches Denken und Handeln. Nach Oerter (1997: 35) ist das komplexe und dialektische Denken bei Heranwachsenden diesen Alters noch nicht ausreichend entwickelt. Jugendliche verfügen naturgemäß auch über weniger politisches Wissen als Erwachsene, was die politische Denk- und Entscheidungsfähigkeit - und damit eine umfassende politische Identitätsbildung einschränkt (Oerter 1997: 38).

\subsubsection{Das ,Recency'-Modell}

Das sogenannte ,Recency'-Modell geht vom Erwachsenenalter als der entscheidenden Lebensphase für die polische Identitätsbildung aus (vgl. Weissberg 1974: 29).

Erst im Erwachsenenalter sind die kognitiven Fähigkeiten voll ausgeprägt und die Menschen in der Lage, die Tragweite politischer Entscheidungen auch für

\footnotetext{
7 Dies heißt allerdings nicht, dass alle Jugendlichen und Erwachsenen diesen Entwicklungsstand erreichen; die Fähigkeit zu formal-logischem Denken ist bei Personen mit hoher Schulbildung stärker ausgeprägt als bei solchen mit geringerer Schulbildung (Oerter 1997: 38).
} 
das eigene Leben zu erkennen. Politische Lernerfahrungen sind demnach um so relevanter, je älter die Heranwachsenden werden.

Vor allem, wenn Politik über eigenes Handeln - wie Wahlverhalten oder politische Gespräche - direkt erfahren wird und kognitiv verarbeitet werden kann, entwickeln sich nach dem ,Recency'-Modell stabile politische Orientierungen und Verhaltensweisen. Frühe Lernerfahrungen der Kindheit und Jugend werden somit relativ leicht vergessen bzw. durch spätere Einflüsse modifiziert.

Alle drei beschriebenen Modelle des politischen Lernens haben für Weissberg (1974:27) einen eigenen spezifischen Erklärungswert, da sie jeweils bestimmte Aspekte des politischen Lernens aufgreifen. Er argumentiert, dass die politische Sozialisation nicht auf bestimmte Altersphasen begrenzt werden können, sondern eine Integration der drei Modelle am fruchtbarsten sei: „Wenn man davon ausgeht, dass grundlegende Orientierung früh gelernt werden und ein hohes Maß an Stabilität aufweisen, während andere politische Orientierungen kontinuierlich gelernt und modifiziert werden und schließlich eine ganze Reihe von Einstellungen und Verhaltensweisen eine Reaktion auf die Stimuli im Erwachsenenalter sind, so haben alle drei Modelle ihren Erklärungswert" (vgl. Weissberg 1974, Wasmund 1982).

\subsubsection{Das Modell der politischen Sozialisation nach Weiß}

Ein Ansatz, der die unterschiedlichen Alters- bzw. Entwicklungsphasen und Sozialisationsinstanzen des Lebensverlaufs berücksichtigt und in einem theoretischen Modell der politischen Sozialisation integriert, wurde von Weiß (1981) formuliert.

Weiß unterscheidet im Prozess der politischen Sozialisation eine primäre Sozialisationsphase, in der vor allem die latente Sozialisationsentwicklung stattfindet, und eine sekundäre Sozialisationsphase. In der primären Sozialisationsphase im Kindes- und frühen Jugendalter findet nach Weiß die Entwicklung der ,Grundpersönlichkeit' statt. Diese Grundpersönlichkeit strukturiert 
die politische Persönlichkeit im späteren Lebensverlauf vor, determiniert sie aber nicht vollständig (Weiß 1981: 51f.).

Als politisch relevanten Aspekt der Grundpersönlichkeit nennt Weiß die sogenannte Selbst- und Sozialkompetenz. Sie fungiert als Prädiktor des allgemeinen sozialen Verhaltens und umfasst neben der Handlungskompetenz (Selbstdarstellung, Empathie, Frustrationstoleranz und Rollendistanz), die kognitive sowie moralisch/emotionale Kompetenz einer Person. Inhaltlich meint die Selbst- und Sozialkompetenz „die Fähigkeit, in Situationen des sozialen Handelns sowohl die eigenen Bedürfnisse einzubringen, als auch die der Interaktionspartner zu berücksichtigen“ (Weiß 1981: 48).

Die Entwicklung der Selbst- und Sozialkompetenz findet in Interaktion mit den Instanzen der primären Sozialisation, insbesondere der Familie, sowie später der Peers bzw. der Schule statt (vgl. Kapitel 3) und bildet die Grundlage für die spätere, sich in der sekundären Sozialisation ausbildenden ,politischen Kompetenz'.

Die sekundäre politische Sozialisation, das heißt die politische Sozialisation im engeren Sinne, setzt nach Weiß erst etwa im Alter von 12 Jahren ein.

Etwa zu diesem Zeitpunkt sind die für die Bildung der ,politischen Kompetenz' relevanten kognitiven Voraussetzung entwickelt, so dass auch manifeste politische Lernerfahrungen wirksam werden können. Im Verlauf der sekundären Sozialisationsphase entsteht dann die individuelle politische Kompetenz und wird - zumindest vorläufig- festgelegt. Die politische Kompetenz bildet sich auf Basis der bereits entwickelten Selbst- und Sozialkompetenzen aus, und umfasst - analog zur Selbst- und Sozialkompetenz - politische Kenntnisse, Einstellungen und Aktivitätsbereitschaft (politische Beteiligung und Verantwortung, Toleranz und Gesellschaftskritik, vgl. Weiß 1981: 50).

In dieser Phase der politischen Sozialisation verändert sich die Bedeutung der Familie. Zwar hat die Familie noch einen wichtigen Einfluss auf die politischen Orientierungen der Kinder, gleichzeitig geht ihr Einfluss mit dem Älterwerden der Heranwachsenden jedoch deutlich zurück. Die Interaktion mit anderen Sozialisationsinstanzen wie Schule, Peers, Medien und später Hochschule oder Arbeitswelt gewinnt dann für die Heranwachsenden und jungen Erwachsenden an Bedeutung (Weiß 1981: 50).

In der Kindheit findet somit eine latente politische Sozialisation statt, während mit dem Erreichen des Jugendalters die manifeste Sozialisation beginnt 
und die Heranwachsenden sich bewusst mit politischen Inhalten und Themen beschäftigen. Die sekundäre Phase der politischen Sozialisation baut dabei auf den Sozialisationserfahrungen der Kindheit auf und kann daher nicht unabhängig von ihr betrachtet werden.

Politische Orientierungen und Handlungen lassen sich für Weiß (1981: 51) demzufolge nur begreifen, wenn sowohl primäre als auch sekundäre Sozialisationserfahrungen berücksichtigt werden. Politische Orientierungen und politisches Verhalten sind demnach nicht ohne kindliche Lernerfahrungen und ebenso wenig ohne Sozialisationserfahrungen nach der Kindheit zu erklären ${ }^{8}$.

\footnotetext{
${ }^{8}$ So vertritt Franklin (1984) die Auffassung, dass die Parteiidentifikation nicht in den frühen Lebensjahren der Kindheit, sondern eher in der Jugend erworben wird. Sie kann aber auch später in der aktiven Auseinandersetzung mit der politischen Umwelt erheblichen Modifikationen unterliegen. Daher sei es - so Franklin (1984) - nicht möglich, das genaue Alter der politischen Sozialisation von Parteiidentifikation zu benennen. Seiner Ansicht nach dürfte es die aber Jugendzeit sein, in der sich Personen der politischen Streitfragen, Probleme und Zusammenhänge bewusst werden.
} 


\section{Politische Sozialisationsinstanzen im Lebensverlauf}

\subsection{Politische Sozialisationsinstanzen}

Als wesentliche Instanzen der politischen Sozialisation werden in der klassischen Sozialisationsforschung Familie, Schule, Peers und Medien genannt. Die Fokussierung auf diese vier Instanzen wird in erster Linie damit begründet, dass diesen Lebensräumen - qualitativ wie quantitativ - die größte Bedeutung zukommt. Außerdem steht die Sozialisationsforschung vor der Schwierigkeit, dass nicht alle möglicherweise relevanten Umwelteinflusse erfassbar sind.

Claußen unterscheidet vier verschiedene - unterschiedlich bedeutsame Instanzbereiche (vgl. Claußen 1996).

Der erste Bereich beinhaltet die primären Sozialisationsinstanzen. Als zentral nennt Claußen hier hauptsächlich Familie (oder familienähnliche Lebensgemeinschaften), Schule, Gleichaltrigengruppe, Medien und Arbeitsplatz (vgl. Claußen 1996: 31f.). Kennzeichnend für diese Lebensbereiche ist, dass die meisten Heranwachsenden dauerhaft - oder zumindest über längere Zeit - in ihrem Kontext leben. Außerdem treten diese Instanzen im allgemeinen nicht in strenger Reihenfolge in Erscheinung. Sie existieren vielmehr oft nebeneinander und substituieren sich phasenweise. Den genannten Sozialisationsinstanzen werden die sog. Primär- und Verstärkungseffekte zur Grundlegung der politischen Identitätsbildung zugeschrieben, d.h. Kinder und Jugendliche entwickeln in der Auseinandersetzung mit innen erste Fragmente und grundlegende Muster der politischen Identität (Claußen 1996: 34).

Neben diesen primären Sozialisationsinstanzen gibt es nach Claußen eine zweite bedeutsame Gruppe von Lebensbereichen. Dazu zählen u.a. Jugendfreizeitstätten, Wehr- oder Zivildiensteinrichtungen, Hochschulen aber 
auch Sport oder Selbsthilfegruppen. Charakteristisch für diese Sozialisationsinstanzen ist, dass sie erzieherische oder ,quasi-erzieherische' Aufgaben übernehmen, die direkt oder indirekt das politische Lernen und das Verhältnis zur Politik berühren. Allerdings unterliegen ihrem Einfluss nicht alle Menschen gleichermaßen und ihre jeweilige Bedeutung ist in der Regel zeitlich begrenzt (Claußen 1996: 33).

Die dritte Gruppe der an der politischen Sozialisation beteiligten Instanzen bezeichnet Claußen (1996: 34) als ,flankierende Sozialisationsinstanzen'. Damit sind allgemeine Lebensumstände ohne eigenen Ort gemeint, von denen eine strukturierende Funktion auf politische Orientierungen angenommen wird. Zu den wichtigsten Instanzen dieser Gruppe gehören die generellen politisch-ökonomischen Rahmenbedingungen und die damit verknüpften Konsum- und Freizeitwelten, die individuelle wirtschaftliche Lage sowie das Leben in verschiedenen religiösen und ethnischen Gemeinschaften.

Als vierte Gruppe mit politischer Sozialisationsfunktion nennt Claußen (1996: 35) Elemente und Kontexte des politischen Systems, an denen allerdings nur sehr geringe Teile der Bevölkerung beteiligt sind: Parteien, parteinahe Institutionen, Bürgerinitiativen oder Einrichtungen wie Umwelt- oder Menschenrechtsverbände etc. .

Im folgenden werden insbesondere die Sozialisationsinstanzen Familie und Schule näher betrachtet, da sie für die anschließende empirische Untersuchung von besonderer Bedeutung sind. Außerdem werden die wesentlichen empirischen Befunde zu den Instanzen Peers und Medien wiedergegeben. 


\subsubsection{Familie}

Lange Zeit wurde die Familie als die mächtigste und einflussreichste Sozialisationsinstanz im Prozess der politischen Identitätsbildung angesehen (vgl. Geißler 1996: 51f., Wasmund 1982a). Bis in die 70er Jahre hinein galt die These vom ,Primat der Familie' als eine Grundannahme der Sozialisationsforschung (Geißler 1996: 52). Theoretisch stützt sich die Betonung der Familie auf psychologisch orientierte Modelle des frühen Lernens (Greenstein 1965, s. auch Kapitel 2) und lerntheoretische Konzepte, die in der frühen Kindheit die Grundlage für politisches Lernen sehen (Hess/Torney 1970). Der Familie wird hier eine Schlüsselfunktion zugeschrieben, da - so die Vorstellung - die früh geprägten politischen Basisorientierungen langfristig wirksam sind und die politischen Orientierungen und Verhaltensweisen im Erwachsenenalter beeinflussen (,Kristallisationsthese', vgl. Zängle 1978: 43f.). Da geeignete Longitudinaldaten, die in früher Kindheit beginnen und einen mehrere Jahrzehnte langen Zeitraum umfassen, bislang fehlen, steht eine stringente empirische Überprüfung dieser These bisher aus.

Im Hinblick auf die Rolle der Familie im Prozess der politischen Sozialisation wird zumeist zwischen latentem und manifestem politischen Lernen unterschieden. Einen wichtigen Beitrag zur Untersuchung latenter politischer Sozialisationseinflüsse in der Familie liefert die Forschung zu Autoritarismus bzw. Faschismus, die seit den klassischen Studien zur ,autoritären Persönlichkeit' (Adorno et al. 1950) bis heute eine Reihe von Studien hervorgebracht hat (Oestereich 1993, Rebenstorf et al. 2000, Rieker 2007, vgl. für Überblicksdarstellungen Geißler 1996, Hopf/Hopf 1997).

Die in der psychoanalytischen Tradition stehende Autoritarismusthese geht davon aus, dass ,kaltes' und strafendes Erziehungsverhalten der Eltern zu Unterwürfigkeit und Aggressionen führt, die von den Eltern auf andere Objekte übertragen werden (vgl. zusammenfassend Geißler 1996, Hopf/Hopf 1997, Rebenstorf et al 2000). Die empirischen Ergebnisse zum Zusammenhang zwischen elterlichem Erziehungsverhalten bzw. Familienklima und politischen Orientierungen sind z.T. widersprüchlich und 
wurden nicht selten in Zweifel gezogen (insbes. Untersuchungen zum autoritären Charakter, vgl. Geißler 1996: 60). Insgesamt aber gibt es deutliche Hinweise darauf, dass politische Einstellungen und Verhaltensweisen der Jugendlichen auch vom Familienkontext abhängen (Frenkel-Brunswik 1996, Hopf 2000, Rebenstorf 2000, Buhl 2003, Rieker 2007).

Für die Autoritarismusforschung besteht ein besonderes methodisches Problem darin, dass autoritäre Menschen dazu tendieren, die Eltern und ihre Beziehung zu ihnen rückblickend zu idealisieren. Negative Erfahrungen im Elternhaus werden häufig bagatellisiert oder ganz geleugnet (Adorno 1969, Hopf/Hopf 1997: 28), so dass es tendenziell schwierig ist, bedeutsame Korrelationen zu ermitteln. In einer Übersicht zur politischen Sozialisation in der Familie bis Mitte der 90er Jahre kommt Geißler jedoch zu dem Ergebnis, dass Studien, die das Familienmilieu differenziert erfassen, zeigen, dass strenge und restriktive Erziehung die Entwicklung autoritärer Eigenschaften begünstigt (Geißler 1996: 60). Nach qualitativen Untersuchungen gibt es Hinweise darauf, dass bei Jugendlichen mit ,passiven' oder ,wütend/konflikthaft verstrickten' familiären Bindungserfahrungen rechtsextreme Orientierungen stark ausgeprägt sind, während Jugendliche, die einen ,sicher-autonomen' Bindungsstil erfuhren, keine rechtsextremen Einstellungen aufweisen (Hopf et al. 1995). Rieker (2007) kommt in einer weiteren qualitativen Untersuchung zum Thema ,fremdenfeindliche Jugendliche' zu dem Schluss, dass fremdenfeindlich eingestellte junge Männer in ihrer Kindheit weniger Zuwendung und mehr Zurückweisung durch ihre Eltern erfuhren (Rieker 2007: 33). Der Familie kommt demzufolge bei der Entwicklung fremdenfeindlicher Orientierungen eine besondere Bedeutung zu. Allerdings nehmen die Einflüsse der Familie ab, wenn andere Sozialisationsinstanzen wichtig werden (Rieker 2007: 38).

Ein ähnliches Bild zeichnen neuere quantitative Untersuchungen. Sie belegen den Zusammenhang zwischen familialen Merkmalen wie Erziehungsstil oder Familienklima und der Entwicklung der politischen Identität von Jugendlichen (Rebenstorf 2000, Buhl 2003). Rebenstorf et al. (2000: 52) konnten beispielsweise in einer Analyse zum Einfluss des elterlichen Erziehungsstils 
auf verschiedene Persönlichkeitsmerkmale zeigen, dass ein signifikanter Zusammenhang zwischen autoritär-rigidem Erziehungsstil der Eltern (bzw. der Mütter) und dem Autoritarismus von Jugendlichen besteht. Eine andere Studie von Buhl (2003: 163f.) untersucht die Bedeutung der Familie für die Entwicklung politischer Orientierungen und Handlungsdispositionen von Heranwachsenden. Sie ermittelt eine signifikante Korrelation $(+0.32)$ zwischen positiven Merkmalen des Familienklimas (harmonisches Familienklima und demokratisches elterliches Erziehungsverhalten) und Legitimitätsbewertung des politischen Systems. Ein negativer Zusammenhang (-0.25) besteht dagegen zwischen konfliktreichem Familienklima und der Bewertung der Systemlegitimität.

Auch in anderen Bereichen der Sozialisationsforschung wurden latente Sozialisationseinflusse der Familie untersucht. Eine Studie zur Aktivitätsbereitschaft von Jugendlichen z.B. ergab einen deutlichen Zusammenhang zwischen dem Grad der Toleranzeinübung (Komplexitätstoleranz, Problemtoleranz, Konflikttoleranz sowie Unsicherheitstoleranz) im Familienkontext und der Bereitschaft zu politischer Aktivität: Die Aufgeschlossenheit gegenüber dem politischen Bereich steigt mit dem Ausmaß, in welchem sich ein Jugendlicher intellektuellen Anforderungen gewachsen fühlt, Problemen nicht aus dem Wege geht, zur kontroversen Kommunikation bereit ist und selbstsicher genug ist, unsichere Situationen ertragen zu können (Schulze 1977: 36).

Zusammenhänge zwischen elterlichen Erziehungsverhalten und politischer Orientierung der Kinder zeigen auch neuere Jugendstudien (Jugendwerk der Deutschen Shell 2002: 77). Politisch interessierte Jugendliche berichten demnach über ein höheres Zutrauen der Eltern in ihre Kinder als politisch nicht interessierte Jugendliche. Auch höhere elterliche Leistungsanforderungen und die Förderung der kindlichen Eigenständigkeit von Seiten der Eltern steht in positiver Beziehung zur Höhe des politischen Interesses der Jugendlichen (Jugendwerk der Deutschen Shell 2002: 84f.). Andere Elternmerkmale zeigen dagegen eine negative Korrelation zum jugendlichen Interesse an Politik: Wurden die Eltern als ,ängstlich-besorgt' 
beschrieben, war das Politikinteresse der Jugendlichen geringer (J ugendwerk der Deutschen Shell 2002: 82). 
Ein wichtiger Forschungsstrang in der politischen Sozialisationsforschung beschäftigt sich mit der Übereinstimmung politischer Orientierungen von Eltern und Kindern (Tedin 1974, 1980; Schulze 1977, Wasmund 1982a, Geißler 1996). Übereinstimmende Einstellungen zwischen Eltern und Kindern bzw. Jugendlichen werden als Folge von Transmissionseffekten gesehen, die auf Akkumulation bzw. Identifikation basieren (Hopf/Hopf 1997: 136f.). Da nur selten geeignete Längsschnittstudien verwendet werden, liegen Aussagen über die Richtung der Beeinflussungseffekte jedoch kaum vor ${ }^{9}$. Prinzipiell sind neben Übertragungen von Eltern auf Kinder auch retroaktive Beeinflussungsprozesse zwischen Eltern und Kindern plausibel - insbesondere mit steigendem Alter und hohem politischen Aktivitätsniveau der Kinder.

Studien, die familiale Übereinstimmungen $\mathrm{zu}$ politischen Themen untersuchen, zeigen, dass das Ausmaß der politischen Homogenität zwischen Eltern und Kindern vom Grad der Politisierung des Elternhauses abhängt. Je häufiger im Elternhaus über politische Themen gesprochen wird und die elterlichen politischen Positionen und Präferenzen für die Kinder sichtbar werden, um so größer sind die politischen Übereinstimmungen zwischen Eltern und Kindern (1982a: 36). Insbesondere dann, wenn sich die Eltern in ihren politischen Positionen einig, die Kinder also homogenen Einflüssen ausgesetzt sind, steigt die Chance, dass die Kinder die elterlichen Positionen übernehmen (Wasmund 1982a, Geißler 1996: 59, Grob 2006).

Dieser Befund gilt vor allem bei grundsätzlichen politischen Orientierungen wie Parteipräferenzen (Jennings/Niemi 1974, Allerbeck/Hoeg 1985), ideologischen Orientierungen (Allerbeck/Hoeg 1985) oder auch fremdenfeindlichen Einstellungen (Rieker 2007, vgl. Geißler 1996). Bei

\footnotetext{
${ }^{9}$ Eine neuere Analyse von Schmid, die u.a. Wechselwirkungsprozesse untersucht, basiert auf einer Längsschnittstudie mit Brandenburger Gymnasiasten (von der 10. bis zur 13. Schulklasse mit insgesamt vier Panelwellen). Die Ergebnisse deuten zwar auf Wechselwirkungsprozessen hin, aber es zeigte sich auch eine Vielzahl an methodischen Problemen, die unter anderem auf niedrige Fallzahlen zurückzuführen sind (Schmid 2001: 181).
} 
spezifischeren und aktuelleren politischen Orientierungen scheint die ElternKind-Homogenität dagegen nur gering zu sein (vgl. Wasmund 1982a).

Von großer Bedeutung ist hier auch das Familienklima (s. auch Kapitel 2): Übereinstimmungen zwischen Eltern und Kindern sind um so ausgeprägter und langfristiger, je besser die Eltern-Kind-Beziehung beurteilt werden und je dichter die Kommunikation in der Familie insgesamt ist. Einzelne Ergebnisse weisen zudem darauf hin, dass die Einstellungen der Mütter für die Kinder relevanter sind als die der Väter, auch wenn eher der Vater als politischer Ratgeber und Kommunikationspartner genannt wird (Grob 2006, Rippl 2004, Rieker 2007, vgl. Geißler 1996).

Manifeste politische Sozialisation kann sich sowohl intendiert als auch nicht intendiert vollziehen. Sie beginnt bereits in der frühen Kindheit, gewinnt aber erst in späteren Sozialisationsphasen an Relevanz. Von besonderer Bedeutung sind dabei der Grad der Politisierung und das familiale Kommunikationsverhalten im Elternhaus. Politische Gespräche in der Familie und das Aktivitätsniveau im Elternhaus weisen systematische Zusammenhänge mit dem politischen Interesse, den Einstellungen zur politischen Effizienz und der Aktivitätsbereitschaft der Kinder auf (Schulze 1977, Kuhn/Oswald 2001: 23).

Insgesamt lässt sich zur politischen Kommunikation in der Familie allerdings feststellen, dass politische Themen nicht im Zentrum der Familiengespräche stehen und Eltern die politische Bildung ihrer Kinder nicht als ihre primäre Aufgabe ansehen (Wasmund 1982a: 35, Geißler 1996: 56, vgl. auch van Deth 2000).

Schichtspezifische Einflüsse dürfen in diesem Zusammenhang aber nicht übersehen werden. Sowohl Quantität als auch Intensität politischer Gespräche hängen deutlich vom sozio-ökonomischen Status der Eltern ab. Vor allem ein höheres Bildungsniveau der Eltern erleichtert den kindlichen Zugang zur Politik (Geißler 1996: 55).

Insgesamt lässt sich sagen, dass die meisten neuen Studien zum Zusammenhang zwischen erlebtem Familienmilieu und den verschiedenen Dimensionen politischen Lernens sich auf Heranwachsende jugendlichen 
Alters beziehen. Seit dem Ende der 70er Jahre beschäftigen sich Untersuchungen nur selten mit dem Familieneinfluss auf kindliche (vor-) politische Orientierungen. Diese Lücke versucht das Projekt „Demokratie leben lernen“ zu schließen, das die politischen Orientierungen von 6- bis 7jährigen Grundschulkindern untersucht (vgl. van Deth 2005: 5). Auf der Basis erster umfangreicher qualitativer Interviews kommen Barton und Schäfer (2005: 35) zu dem Ergebnis, dass Kinder "... grundsätzlich über ein gewisses politisches (Vor-) Verständnis, Grundorientierungen und Wissen verfügen. Sie können mit politischen Inhalten umgehen, sind politisch involviert und interessiert“.

\subsubsection{Schule}

Kraft ihres Bildungsauftrags wird der Schule eine besondere Aufgabe im Prozess der politischen Sozialisation von Kindern und Jugendlichen zugewiesen. Sie hat - zumindest in demokratischen Systemen staatlicherseits den Auftrag, die Entwicklung zum ,mündigen Bürger' zu fördern und zur "Sicherung der mentalen Infrastruktur einer civil society“ (Fend 2006: 400) beizutragen. ${ }^{10}$

10 Dies wird in der Realität allerdings nicht immer umgesetzt (vgl. Fend 2006: 401). Wie empirische Forschungsergebnisse der Politikwissenschaft belegt haben, wird die Idealform des politisch mündigen Bürgers auch von erwachsenen Bürgern demokratischer Systeme kaum erreicht. So wurde z.B. gezeigt, dass viele Bürger selbst über einfache Sachverhalte nur schlecht informiert sind und unzureichende kognitive Strukturen ausgebildet haben (Berelson et al. 1954), ihr Kenntnisstand über politische Strukturen und politische Prozesse relativ niedrig ausfällt und weit von den geforderten Normen entfernt ist (Almond/Verba 1965; Delli Carpini/Keeter 1996). Insofern ist auch bei den Jugendlichen - besonders in Anbetracht der notwendigen kognitiven Verarbeitungsleistungen, die erbracht werden müssen - noch eher mit Zurückhaltung, ambivalenten Einstellungen und Verhaltensweisen sowie Orientierungsproblemen gegenüber dem politischen Bereich zu rechnen (siehe auch Kuhn 2000: 22). 
$\mathrm{Zu}$ den wesentlichen schulischen Bildungszielen zählen demzufolge (Wasmund 1982b: 65):

- der Erwerb politischer Kenntnisse

- die Identifikation mit den demokratischen Werten

- die Bereitschaft, das politische System zu unterstützen und sich politisch zu beteiligen

- die Fähigkeit, politische Institutionen, Prozesse und die handelnden politischen Eliten kritisch zu beurteilen

Wie in den anderen Lebensbereichen auch, finden die politischen Lernprozesse in der Schule sowohl auf manifester als auch auf latenter Ebene statt.

Zum manifesten politischen Lernen in der Schule gehört in erster Linie die Wissensvermittlung im Politik- oder Sozialkundeunterricht. Aber auch in benachbarten Fächern wie Geschichte, Geographie oder Deutsch werden politische Themen behandelt (Ackermann 2002: 450).

Neben den manifesten Lernzielen, die in den schulischen Lernplänen formuliert sind, tragen die latenten politischen Lernerfahrungen in der Schule zur politischen Sozialisation der Schülerinnen und Schüler bei. Dazu gehören verschiedene Aspekte der, gelebten politischen Kultur' im Schulkontext (Fend 2006: 395), die auf das Stichwort ,heimlicher Lehrplan' verweisen und die Persönlichkeitsentwicklung der Kinder und J ugendlichen mitgestalten.

Konkret zählen zu den latenten Lernerfahrungen Art und Qualität der LehrerSchüler-Beziehungen, institutionellen Rahmenbedingungen und schulische Mitbestimmungsmöglichkeiten sowie das soziale Klima und die Regeln des alltäglichen Umgangs miteinander (vgl. Kandzora 1996, Fend 2006).

Aus normativer Sicht können bzw. sollten die Elemente einer, gelebten politischen Kultur' den Schülerinnen und Schülern Gelegenheit geben, demokratisches Leben erfahrbar zu machen und einzuüben (Fend 2006: 395). 
Allerdings stehen jene Faktoren, die Schule als ,Lehranstalt' und weniger als ,Lernort' charakterisieren, dem Ziel zur Erziehung ,mündiger Bürger' entgegen. Kandzora (1996: 75f.) beschreibt die Bedeutung der impliziten schulischen Sozialisationsprozesse folgendermaßen: „Betrachtet man die Schule im Foucaultschen Sinne als staatlichen Machtapparat, dann rücken all diejenigen Elemente des, heimlichen Lehrplans' ins Blickfeld, die auf eine Einübung in gesellschaftliche Hierarchien abzielen sowie insgesamt auf eine Domestizierung des einzelnen zu einem funktionalen Element reibungsloser gesellschaftlicher Abläufe“.

Solche Elemente des ,heimlichen Lehrplans' sind u.a. (Kandzora 1996: 71):

- der Pflichtcharakter schulischen Lernens

- die Asymmetrie zwischen Lehrern und Schülern

- die Reduktion von der Schülerpersönlichkeit vor allem im Bereich der Emotionen und des Handelns

- die Anpassung an Lehrererwartungen

- der bürokratisierte Ablauf

- die Ausbildung von Sekundärtugenden wie Gehorsam, Pünktlichkeit, Ordnung und Leistungsorientierung

- das Einüben von Konkurrenzverhalten

Anhand dieser Liste wird deutlich, dass die Ausbildung einer unabhängigen Persönlichkeit, die fähig und willens ist, die politischen Institutionen, Abläufe und handelnden Eliten kritisch zu beurteilen und aktiv am politischen Prozess teilzuhaben, unter den gegebenen schulischen Bedingungen mit Schwierigkeiten verbunden ist und leicht zu Konflikten führt.

Dementsprechend kommt Wasmund (1982b: 65) nach Durchsicht empirischer Untersuchungen bis zum Ende der 70er Jahre zu dem Ergebnis, dass die schulische politische Bildung in der Praxis nicht einmal ihrer primären Aufgabe einer grundlegenden politischen Wissensvermittlung gerecht wird (Wasmund 1982b: 65). Dieser Befund gilt jedoch nicht nur für 
die Bundesrepublik: Studien wie etwa die international vergleichenden IEA (International Association for the Evaluation of Educational Achievement)Studie "Civic Education in Ten Countries"11 machen darauf aufmerksam, dass die bildungspolitischen Ziele - d.h. die Erziehung der Schüler zu politisch informierten, systemunterstützenden und zugleich demokratisch eingestellten und teilnahmebereiten Bürgern - in keinem der teilnehmenden Ländern erreicht wurde (Torney et al. 1975, Wasmund 1982b: 65).

Die IEA-Studie macht aber auch deutlich, dass ein offenes und vertrauensvolles Diskussionsklima, in dem die Jugendlichen ermutigt werden, ohne Scheu zu diskutieren, die Entwicklung der politischen Effektivität und Beteiligungsbereitschaft fördert (vgl. Torney et al. 1975; Oesterreich 2002).

Die Ergebnisse konnten in der Folgestudie „Citizenship and Education in Twenty-eight Countries“ für das Jahr 1999 grundsätzlich bestätigt werden ${ }^{12}$.

Die Untersuchung ergab, dass die Schule am ehesten in der Lage ist, politisches Wissen in Form von Grundbegriffen über politische Institutionen zu vermitteln. Ein differenziertes Verständnis demokratischer Werte und Prinzipien konnte dagegen bei den Jugendlichen nur sehr selten diagnostiziert werden.

Auch hier zeigte sich der förderliche Einfluss eines offenen und demokratischen Schulklimas. Außerdem wurde deutlich, dass in vielen Ländern eine Beteiligung an schulischen Mitbestimmungsorganen wie dem Schülerparlament oder der Schülermitverwaltung einen positiven Einfluss auf politisches Wissen und das spätere politische Engagement hat (vgl. Torney et al. 1975; Oesterreich 2002).

Ebenfalls mit Daten des „Civic Education“- Projektes führte Watermann eine Mehrebenenanalysen zur Wirksamkeit diskursiven Unterrichtsstils durch

\footnotetext{
11 Bei dieser Studie handelt es sich um eine in zehn Ländern an etwa 30.000 Schülern durchgeführte Untersuchung zur politischen Bildung 10-18-jähriger Jugendlicher.

12 Die Folgestudie fand 1999 statt und untersuchte fast 90.000 Jugendliche in 28 Ländern im Alter von etwa 14 Jahren im Hinblick auf politisches Wissen und Engagement.
} 
(Watermann 2003). Er konnte zeigen, dass der Unterrichtsstil einen eigenständigen Effekt auf politisches Verständnis und demokratische Einstellungen ausübt, der auch dann erhalten bleibt, wenn für die Schulform kontrolliert wird (Watermann 2003: 367)

$\mathrm{Zu}$ ähnlichen Ergebnissen kommen auch neuere Untersuchungen aus dem Bereich der Jugendforschung. So zeigen die Daten des Jugendsurveys des Deutschen Jugendinstitut (DJI) einen hoch signifikanten Zusammenhang zwischen schulischen Mitwirkungsmöglichkeiten und der politischen Teilnahmebereitschaft bei Unterschriftenaktionen bzw. Demonstrationen (Alt et al. 2005: 31).

Insgesamt wird aber der Schule - trotz ihrer institutionellen politischen Bildungsaufgabe - im Vergleich mit anderen Sozialisationsinstanzen eine eher geringe Bedeutung zugeschrieben (vgl. Ackermann 1996 und 2002). Dieser

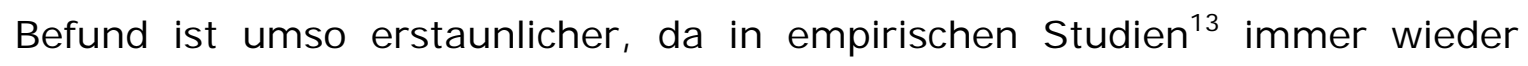
bestätigt wurde, dass das formale Bildungsniveau einer der stärksten Prädiktoren für ein hohes politisches Aktivitäts- und Wissensniveau ist (vgl. Almond/Verba 1989, van Deth 1989, Hajdar/Becker 2006): Höher Gebildete nutzen die Medien häufiger zur politischen Informationsbeschaffung, kommunizieren häufiger über Politik und weisen ein stärkeres politisches Kompetenz- und Effektivitätsniveau sowie eine höhere Partizipationsbereitschaft auf (vgl. Almond/Verba 1989: 22f., Kaase 1989: 37f.). Empirische Jugend- und Schülerstudien der letzten Jahre zeigen auch deutliche Unterschiede zwischen Schülern verschiedener Schultypen (vgl. z. B. Fend 1991, Hoffmann-Lange 1995, Westle 2006). So spricht Fend etwa von einer starken Schere in der politischen Urteilsfähigkeit zwischen Hauptschülern und Gymnasiasten. Er konnte zeigen, dass schon 13-jährige Gymnasiasten ein besseres Verständnis für demokratische Prozesse

\footnotetext{
13 Hier sind z.B. die ,Political Action'-Studien und die ,The Civic-Culture'-Untersuchungen zu nennen. Oder in jüngerer Zeit die Untersuchungen, die aus dem von der Deutschen Forschungsgemeinschaft geförderten Projekt „politische Einstellungen, politische Partizipation und Wählerverhalten im vereinigten Deutschland“ hervorgegangen sind, vgl. Falter et al. 2000.
} 
ausgebildet hatten als Hauptschüler im Alter von 15 Jahren (Fend 1991: 175). 
Für die Erklärung dieses Phänomens kommen verschiedene Ansätze in Frage (vgl. auch Kuhn 2000: 59):

- die längere und qualitativ anspruchsvollere Ausbildung im Gymnasium erleichtert die Ausbildung kognitiver Kompetenzen und Wissensstände, die für die Entwicklung eines, günstigen' politischen Orientierungsmusters von Vorteil sind

- durch das Bildungsmoratorium im Gymnasium und in der Oberstufe - fernab von Zwängen der Arbeitswelt - werden Freiräume für die Auseinandersetzung mit gesellschaftlichen Problemen geschaffen

- Gymnasiasten die eine Oberstufe besuchen, haben länger die Möglichkeit, schulische Mitbestimmung zu erleben und einzuüben und so politische Handlungskompetenzen zu entwickeln

- Schüler, die höhere Schulen besuchen, stammen überproportional häufig aus sozial privilegierten Gesellschaftsschichten. Die positiveren politischen Orientierungsmuster der Gymnasiasten können somit durch die Drittvariable ,soziale Herkunft' beeinflusst werden. Auf solche Drittvariableneffekte weisen die Untersuchungen der IEA von 1999 hin (Oesterreich 2002).

Nicht zuletzt aufgrund der hier angesprochenen Überlagerungen von schulischen Effekten mit den Einflüssen anderer Lebensbereiche wie Famile, Peers und Medien ist es grundsätzlich schwierig, direkte Schuleffekte zu belegen bzw. von anderen Einflüssen trennen (vgl. Watermann/Buhl 2003: 340). 


\subsubsection{Peer Group}

Während in der frühen Kindheit in erster Linie die Familie für die politische Identitätsentwicklung bedeutsam ist, werden aus entwicklungspsychologischer Sicht die Kontakte zu Gleichaltrigen und Gleichaltrigengruppen im Jugendalter zunehmend wichtiger (vgl. Hurrelmann 1991, Sünker 1996). Zwar bleibt die emotionale und instrumentelle Funktion des Elternhauses auch im späteren Jugendalter in der Regel weiter bestehen, mit steigendem Alter werden aber Gleichaltrige und jugendliche Subkulturen zu immer wichtigeren Bezugspersonen bzw. Bezugsgruppen ${ }^{14}$ (Schneider 1995: 330, Hopf/Hopf 1997: 154f., Fend 2006: 171, Vetter 2006). Vor allem für die Altersgruppe der 14- bis 20-jährigen ist die Gruppe der Gleichaltrigen der zeitintensivste Ort für Freizeitbeschäftigungen (vgl. Melzer 1992, Fend 2006: 170).

Die Beziehungen zu Freunden und in Cliquen bieten ein wichtiges Lernfeld für den Aufbau grundlegender sozialer Handlungskompetenzen, die auch für die politische Identitätsentwicklung wichtig sind (Krappmann 1991). Im Gegensatz zu den Beziehungen mit Eltern, Lehrern oder anderen Erwachsenen sind Peer-Groups in der Regel nicht a priori hierarchisch organisiert und verfügen über keine festgefügten Rollen - und Positionszuschreibungen. Für Piaget (1954/73) kann die Einübung demokratischer Prinzipien in der Gleichaltrigengruppe erheblich zur Moralentwicklung von Kindern und Jugendlichen beitragen. So lassen sich die Fähigkeit zur Empathie oder Kooperation und die Ausbildung egalitärer bzw. selbstbestimmter Beziehungsmuster in Gleichaltrigenbeziehungen erlernen und trainieren. Auch für die Austragung von Konflikten und das Finden von Kompromissen stellt die Peer-Group ein wichtiges Lern- und Übungsfeld dar (vgl. auch Torney-Purta et al. 1999).

\footnotetext{
${ }^{14}$ Vergleichsstudien, die unter anderem vom Jugendwerk der Deutschen Shell durchgeführt wurden (vgl. Zinnecker 1985, Zinnecker/Fischer 1992), zeigen außerdem, dass es in den letzten 50 Jahren zu einem Bedeutungsanstieg von Cliquen und Freundschaften für die Heranwachsenden gekommen ist.
} 
In der Literatur wird überdies darauf hingewiesen, dass die Bedeutung der Gleichaltrigengruppe vom Alter der Jugendlichen abhängt; verschiedene Längsschnittuntersuchungen an Jugendlichen zeigen: Je älter die Jugendlichen werden, umso wichtiger werden Gleichaltrige auch als politische Gesprächspartner (vgl. Fend 1991 und 2006, Krampen/Ebel 1991, Hopf/Hopf 1997: 157). Empirischen Studien zeigen allerdings auch immer wieder, dass der Bereich ,Gesellschaft und Politik' im Vergleich zu anderen Lebensbereichen einen geringen Stellenwert im Leben der Heranwachsenden einnimmt (vgl. Fend 1991: 264f.). In der "Civic Education Study“ von 1999 etwa gaben nur $14 \%$ der in Deutschland befragten Jugendlichen an, die Gespräche mit dem besten Freund/der besten Freundin seien wichtig für den Erwerb politischer Orientierungen (Torney-Purta et al. 1999).

Ob und wie weit Peer-Groups politische Orientierungen direkt beeinflussen, hängt vom Grad der Politisierung der Gruppe und der Qualität der Beziehungen ab. Die Bedeutung der Peer Group bei manifesten politischen Lernprozessen ist demnach umso größer, je ausgeprägter das politische Interesse der Gleichaltrigen ist und je häufiger über politische Angelegenheiten gesprochen wird. So konnte in den bereits oben erwähnen Analysen des Brandenburger Jugendlängsschnitts (vgl. Kuhn et al. 2001, Schmid 2001: 183) gezeigt werden, dass signifikante politische Übereinstimmungen im Wahlverhalten vor allem bei solchen Jugendlichen zu finden sind, die häufig über Politik diskutieren und sich als ,beste' Freunde bezeichneten (Schmid 2001: 183).

Andererseits können Gleichaltrigengruppen auch indirekt Einfluss auf politische Orientierungen ausüben, indem sie sich bewusst bzw. demonstrativ von der Politik distanzieren und damit zur Entpolitisierung und Demotivierung der Jugendlichen beitragen (vgl. van Deth 1990).

Vergleicht man allerdings die politischen Übereinstimmungen der Jugendlichen untereinander mit denen der Eltern, so fallen jene zwischen den Gleichaltrigen in der Regel etwas niedriger aus (vgl. Campbell 1980, Sebert et al. 1974). 


\subsubsection{Medien}

Dass im Prozess der politischen Identitätsbildung Massenmedien - neben Elternhaus, Schule und Peer Groups - eine wichtige Rolle spielen, wird im allgemeinen nicht bestritten. Allerdings unterscheiden sich die Medien nach Bonfadelli (1981) in einigen zentralen Punkten von den anderen Sozialisationsinstanzen. So werden Massenmedien meist freiwillig und selektiv genutzt und kommunizieren z.T. sehr heterogene oder auch widersprüchliche politische Inhalte. Außerdem ist es mit Hilfe der Medien möglich, geografische, zeitliche und soziale Grenzen zu überwinden, so dass auch ansonsten schwer zugängliche Informationen vermittelt werden können.

Da Politik in der Regel nicht direkt erfahren, sondern meist medial vermittelt wahrgenommen wird, besitzen Medien ein großes politisches Einflusspotential. Hierbei spielen sowohl Aufklärung - d.h. die Vermittlung von politischem Wissen - als auch die Möglichkeiten und Gefahren der politischen Manipulation eine wichtige Rolle (vgl. Pöttker 1996).

Insbesondere die Tageszeitung, das Fernsehen und in zunehmendem Maße auch das Internet - gelten als wichtige Informationsquellen für aktuelle politische Geschehnisse und ihrer Bewertung.

Die vorliegenden empirischen Befunde kommen insgesamt zu einem positiven Ergebnis, was den Zusammenhang zwischen informierender Mediennutzung und politischer Sozialisation anbetrifft (Neller 2002a: 442, Kuhn 2000). Kuhn beispielsweise stellte in einer Studie zum Zusammenhang zwischen Mediennutzung und politischer Sozialisation von Jugendlichen im Alter von 13 bis 20 fest, dass die Häufigkeit der medialen Aufnahme politischer Informationen positiv mit dem politischen Interesse, der politischen Kompetenz und Effektivität sowie mit der politischen Partizipationsbereitschaft korrelierte (Kuhn 2000: 199). Allerdings können über die Kausalitätsrichtung der Zusammenhänge nur schwer Aussagen gemacht werden (Neller 2002a: 442, Ingrisch 1997, Kuhn 2000: 212, Schmid 2004). In einer längsschnittlich angelegten Untersuchung zum politischen Interesse Jugendlicher fand Schmid jedoch zumindest partielle Hinweise auf 
Wechselwirkungsprozesse zwischen medialer Informationsnutzung und dem politischen Interesse der Befragten (Schmid 2004: 153).

Aber auch (scheinbar) ,unpolitische' Medieninhalte wie Unterhaltung oder Werbung können Einfluss auf die politische Identitätsbildung haben. Insbesondere dem Fernsehen wird hierbei eine mächtige und häufig auch dysfunktionale Wirkung auf die Entwicklung von Kindern und Jugendlichen zugewiesen. Holz-Bacha (1989) konnte z.B. Zusammenhänge zwischen der Häufigkeit unterhaltender Mediennutzung und politischer Entfremdung bzw. mangelnder Partizipationsbereitschaft aufzeigen (,These der Video-Malaise‘).

Von Bedeutung sind in diesem Zusammenhang auch Interaktionsprozesse zwischen Medieneinflüssen und den Effekten anderer Sozialisationsinstanzen. So muss von einem Zusammenhang zwischen dem politischen Aktivierungsniveau im Elternhaus und den medialen Sozialisationspotential ausgegangen werden.

\subsubsection{Spätere Sozialisationseinflüsse}

Neben den Sozialisationsinstanzen der Kindheit und Jugend sind auch Sozialisationserfahrungen und Instanzen zu erwähnen, die im späteren Lebensverlauf hinzukommen und Auswirkungen auf politische Orientierungen und politisches Handeln haben können. Die Frage, welche Bedeutung man diesen tertiären Einflüssen zuschreibt, hängt davon ab, welchem theoretischen Modell (,Primacy Principle'-Modell, ,Intermediate Period'-Modell oder ,Recency'- Modell, vgl. Kapitel 2) man folgt. Geht man davon aus, dass die politische Sozialisation mit dem Ende von Kindheit oder Jugend im wesentlichen abgeschlossen ist, dann wird man Erfahrungen des Erwachsenenalters wenig Beachtung schenken. Nimmt man indes an, dass auch Erwachsene noch lernfähig und veränderbar sind, so wird das Augenmerk (auch) auf spätere Lebenserfahrungen gelegt werden (vgl. auch Kapitel 4 zum Thema Lebenszykluseffekte).

Als relevante Sozialisationsinstanz nach der Adoleszenz ist vor allem die Arbeitswelt (bzw. das Studium) zu nennen. Die These, dass berufliche Arbeit 
Einfluss auf die Entwicklung der Persönlichkeit nimmt, ist kein neuer Gedanke, sie geht nicht zuletzt auf Marx und Smith zurück. Empirische Untersuchungen zu diesem Thema wurden insbesondere von Kohn und Mitarbeitern vorgelegt (vgl. Kohn 1981). Kohn zufolge trägt die langfristige Erfahrung beruflicher Autonomie wesentlich zur Ausbildung des ,vorpolitischen' Persönlichkeitsmerkmals Selbstbestimmung (,Self Direktion') bei (Kohn 1981: 203). Fremdbestimmte Arbeitsabläufe und Arbeitsbedingungen, die Gehorsam gegenüber Autoritäten verlangen, bewirken hingegen psychische Dispositionen, die Kohn als ,Anpassung' (,Conformity') bezeichnet (Kohn 1981: 205, vgl. auch das Konzept der ,Autoritären Persönlichkeit'). Ebenfalls erhebliche Folgen für die Persönlichkeit - in Richtung Frustration und Orientierungslosigkeit - wird dem Verlust von Erwerbstätigkeit zugeschrieben (vgl. Steinkamp/Meyer 1996).

Ein weiterer Bereich der politischen Erwachsenensozialisation beschäftigt sich mit Auswirkungen von Partnerschaft, Ehe oder Familienleben auf politische Orientierungen und Verhaltensweisen. So konnten z.B. Burns et al. (1997) in einer Untersuchung zu den Auswirkungen, privater Ungleichheit' zeigen, dass die politische Aktivität stärker ist, wenn Individuen die häuslichen finanziellen Mittel kontrollieren und autonom über ihre Freizeit verfügen können. Dieser Effekt konnte allerdings nur für Männer, nicht aber für Frauen belegt werden: "In short, being boss at home is political empowering to husbands“ (Burns et al. 1997: 373).

Allerdings besteht bei den Untersuchungen, die sich mit späten Sozialisationseffekten befassen, grundsätzlich das Problem, dass die Kausalitätsrichtung der Einflüsse nicht geklärt werden kann, sofern Effekte früherer Lebensphasen nicht kontrolliert werden. Mit Querschnittsuntersuchungen, die zumeist verwendet werden, lassen sich zurückliegende Erfahrungen und Lebensbedingungen allenfalls mithilfe von Retrospektivfragen berücksichtigen (vgl. etwa Becker/Mays 2003). Retrospektivfragen zu früheren Sozialisationseinflüssen- und Instanzen liegen jedoch in Querschnittserhebungen nur selten vor und sind zudem methodisch nicht unproblematisch (vgl. Riemer 2001). 
Die Frage, inwieweit es zu Wechselwirkungen zwischen Familieneinflüssen und den Einflüssen von Peer Group, Schule, Medien und anderen Instanzen bei der politischen Identitätsausbildung der Jugendlichen kommt, ist noch relativ unklar. Anzeichen dafür, dass z.B. Familien- und Gleichaltrigeneffekte zusammenwirken und sich die Einflüsse addieren, findet Schulze (1977: 109f.) in seiner Studie zur politischen Aktivierung Jugendlicher. Danach erfahren Jugendliche aus politisch stärker aktivierten Elternhäusern auch im Kontakt zu Freunden (und in der Schule) stärkere politische Aktivierung durch interessierte und engagierte Gleichaltrige. Es ist somit gut möglich, dass Jugendliche aus politisch involvierten Elternhäusern gezielt Freundschaften mit politisch gleich gesinnten suchen, was ihr Aktivierungsniveau insgesamt weiter ansteigen lässt (vgl. auch Buhl 2003, Schmid 2004). 


\section{Inhalte der politischen I dentität: Politisches I nteresse und Wertorientier- ungen}

Im Zentrum der folgenden Untersuchung stehen zwei Aspekte der politischen Identität, die der affektiv-motivationalen Dimension zugeschrieben werden können (vgl. Kapitel 2): Politisches Interesse und gesellschaftspolitische Wertorientierungen. Es wird davon ausgegangen, dass es sich bei innen trotz gewisser Schwankungen - um stabile Bestandteile der politischen Identität handelt, die sich im Laufe der primären politischen Sozialisation herausbilden.

\subsection{Politisches Interesse}

Politisches Interesse kann mit van Deth (1990: 281) als "degree to which politics arouses a citizen's curiosity" definiert werden. Es umfasst nach dieser Definition Neugier, Aufmerksamkeit und Zuwendung gegenüber politischen Sachverhalten und Geschehnissen. Für Kuhn (2000: 30) beinhaltet politisches Interesse „das Gefühl oder die Einstellung, dass Politik für einen selbst von Bedeutung ist“. Es fördert die Bereitschaft, sich aktiv mit politischen Themen auseinander zu setzen, sich politisches Wissen anzueignen und an Diskussionen mit politischen Inhalten teilzunehmen (vgl. Schmid 2004, Kuhn 2000).

Wie in empirischen Untersuchungen gezeigt wurde, weisen politikinteressierte Bürger stabilere politische Orientierungen und Werte auf als politisch uninteressierte (van Deth 1990). Zudem wird innen ein überdurchschnittlich gut strukturiertes und in sich konsistentes politisches Einstellungsgefüge zugeschrieben (Neller 2002: 490b, vgl. auch Converse 1964). 
In der Partizipationsforschung wird das politische Interesse als eine Vorstufe der politischen Partizipation angesehen. In demokratisch verfassten Staaten gilt es überdies als notwendige Voraussetzung für das Funktionieren des Systems: „Ohne Interesse an Politik gibt es kein politisches Engagement und ohne Beteiligung per definitionem keine Mitbestimmung der Bürger“ (van Deth 2000: 115). Der politisch interessierte, informierte und an den politischen Prozessen aktiv teilnehmende Bürger wird demzufolge in vielen demokratietheoretischen Ansätzen - etwa im Konzept der ,Civic Culture' (Almond/Verba 1963) - als der anzustrebende Idealfall angesehen. Umgekehrt kann ein Mangel an politischem Interesse sowie eine Abkehr der Bürger von der Politik zu ungenügender Kontrolle der politischen Akteure und zu verminderter politischer Beteiligungsbereitschaft der Bürger führen - etwa bei politischen Wahlen (vgl. Barnes et al. 1979)

Aus politikwissenschaftlicher Sicht wird daher fehlendes Politikinteresse als problematisch für die Legitimität eines demokratischen Systems angesehen. Zur Erklärung von politischem Desinteresse hebt van Deth in Anlehnung an Rosenberg drei Aspekte hervor (van Deth 1990: 279 f.): Zum einen kann Entstehung von politischem Interesse und Engagement durch ein soziales Umfeld, in dem Politik ein negatives Image hat, behindert oder sogar sanktioniert werden. Zum zweiten fehlen häufig konkrete Handlungsanreize, um sich mit Politik zu beschäftigen, da politisches Engagement für die meisten Menschen nicht unmittelbar relevant ist. Außerdem kann die Vorstellung oder die Erfahrung, dass politische Aktivität sinnlos ist, behindert die Ausbildung von Politikinteresse bzw. bereits vorhandenes politisches Interesse reduzieren.

Bedingungen, die es für den Bürger lohnender machen, sich mit Politik zu beschäftigen, fördern dagegen das Interesse an politischen Angelegenheiten (van Deth 2000). Ein politischen Inhalten gegenüber aufgeschlossenes soziales Netzwerk, in dem politische Kommunikation und eigenes politisches Handeln erwünscht und gefördert werden, schafft demnach Anreize, sich mit politischen Themen auseinander zusetzen und politisches Interesse herauszubilden (vgl. auch Fend 1991: 153). 


\subsubsection{Determinanten des politischen I nteresses}

Dank umfangreicher Befunde aus der empirischen Jugendforschung lassen sich Ausmaß und Determinanten jugendlichen Politikinteresses recht gut beschreiben (vgl. für Deutschland: Jugendwerk der Deutschen Shell, Jugendstudien des Deutschen Jugendinstituts, vgl. auch Schmid 2004, Brauer 2006).

Für die Ausbildung von politischem Interesse im Jugendalter sind diesen Studien zufolge der Grad der Politisierung und das familiale Kommunikationsverhalten im Elternhaus von besonderer Bedeutung. Als empirisch gesichert gelten die positiven Zusammenhänge zwischen politischen Gesprächen in der Familie bzw. dem politischen Aktivitätsniveau im Elternhaus einerseits und dem politischen Interesse bzw. der politischen Aktivitätsbereitschaft der Kinder und J ugendlichen andererseits (Kuhn/Oswald 2001: 23, Schulze 1977, Schmid 2004). Ist politisches Interesse für die Heranwachsenden im Elternhaus wahrnehmbar und beschäftigen sich die Eltern aktiv mit politischen Themen - indem sie zum Beispiel den politischen Teil der Tageszeitung lesen und darüber kommunizieren - dann fördert dies das politische Aktivitätsniveau der Jugendlichen (vgl. auch die Übersichtsdarstellungen von Wasmund 1982a, Geißler 1996 und Hopf/Hopf 1997).

Aber auch latente politische Sozialisationseinflüsse - wie z. B. das elterliche Erziehungsverhalten - stehen in einem positiven Zusammenhang mit jugendlichem Politikinteresse. Neuere Jugendstudien (Jugendwerk der Deutschen Shell 2002: 77) belegen, dass elterliches Zutrauen in ihre Kinder die Entwicklung des Politikinteresses der Heranwachsenden fördert. Politisch interessierte Jugendliche berichten über mehr elterliche Selbständigkeitsförderung und höhere Leistungsanforderungen als politisch desinteressierte Jugendliche (Jugendwerk der Deutschen Shell 2002: 84f.). Andere Elternmerkmale zeigen hingegen eine negative Beziehung zum Politikinteresse der Nachkommen: Wurden die Eltern als ängstlich und 
besorgt charakterisiert, so äußerten die Jugendlichen stärkeres Desinteresse an Politik (Jugendwerk der Deutschen Shell 2002: 82).

Grundsätzlich spielt auch die individuelle Ressourcenausstattung (Bildung, Zeit, Fähigkeiten) eine Rolle bei der Entwicklung von Politikinteresse (vgl. van Deth 2000: 122, Neller 2002b). Kaase z.B. beschreibt für die Bundesrepublik - beginnend mit dem Wirtschaftswunder und bis in die 80er Jahre des letzten Jahrhunderts hinein andauernd - einen enormen Anstieg des politischen Interesses: der Anteil der insgesamt an Politik interessierten Bürger stieg von zyklischen Schwankungen abgehen - von etwa 30\% zu Beginn der 60er Jahre auf fast 70\% Mitte der 80er Jahre (Kaase 1989) ${ }^{15}$.

Dieser stetige Anstieg des durchschnittlichen subjektiven politischen Interesses in der Bevölkerung wurde in erster Linie mit dem steigenden Bildungs- und Wohlstandsniveau der nachwachsenden Kohorten erklärt (vgl. Kaase 1989, van Deth 1990). Besser gebildete Menschen verfügen über größere intellektuelle Möglichkeiten, komplexe politische Informationen und Zusammenhänge wahrzunehmen, zu verstehen und einzuordnen. Für sie ist die politische Informationsbeschaffung und die Auseinandersetzung mit politischen Themen mit geringern Kosten verbunden als für weniger Gebildete. Hinzu kommt, dass bei größerem politischen Verständnis und Wissen die Bedeutung politischer Geschehnisse für das eigene Leben besser eingeschätzt werden kann. Individuen mit einem höheren Bildungsniveau verfügen somit - was der Entwicklung des Politikinteresses förderlich ist über einen leichteren Zugang zu politischen Themen und über eine bessere Orientierung im politischen Raum (vgl. Nie et al. 1994, Hofmann-Lange 2000, Hajdar/Becker 2006).

Neben dem politischen Interesse ist auch die Ausbildung politischer Kompetenz- und Effizienzwahrnehmung stark bildungsabhängig. Empirische Daten zeigen, dass Menschen die mit einer höheren Bildung ausgestattet sind, sich als kompetenter einschätzen politische Zusammenhänge zu

${ }^{15}$ Gabriel und van Deth (1995: 397) weisen allerdings darauf hin, dass ein solcher Anstieg nicht zu beobachten ist, wenn statt nach dem subjektiven politischen Interesse nach einem anderen Indikator, nämlich der Häufigkeit von politischen Gesprächen mit Freunden, gefragt wird. Sie registrieren seit Mitte der 70er Jahre eine tendenziell eher sinkende politische Gesprächshäufigkeit. 
verstehen und politisch Einfluss zu nehmen (Vetter 2000, Krimmel 2000, Hajdar/Becker 2006).

Als eine weitere wichtige Determinante für die Stärke des politischen Interesses erweist sich bis heute - allen gesellschaftlichen Angleichungsprozesse zum Trotz - immer wieder die Geschlechtszugehörigkeit (vgl. Westle/Schoen 2002, Brauer 2006). Danach werden Frauen in Deutschland - wie auch in anderen Staaten - als weniger politikinteressiert charakterisiert als Männer.

In der Literatur existieren verschiedene Ansätze, um diesen Geschlechtsunterschied zu erklären:

- Zum einen werden Unterschiede in der politischen Sozialisation für die Geschlechterkluft verantwortlich gemacht. Es wird argumentiert, Mädchen würden in ihrer Sozialisation darauf vorbereitet, sich um Haushalt, Kinder und um soziale Beziehungen zu kümmern, während Jungen lernten, sich im Wettbewerb um Macht und Einfluss durchzusetzen. Damit werde - zumindest unterschwellig - die Vorstellung vermittelt, Politik sei ein männliches Betätigungsfeld, das für Frauen nicht von Bedeutung sei bzw. von dem sich Frauen fernzuhalten hätten (vgl. Westle/Schoen 2002). Dementsprechend findet Westle (2006: 232) in einer Untersuchung Nürnberger Jugendlicher im Bereich der latenten politischen Sozialisation auch heute noch „ein geradezu frappierendes traditionelles Muster der heimischen Arbeitsverteilung zwischen den Elternteilen“ (vgl. auch Barton/Schäfer 2005).

- Ein weiterer Ansatz geht davon aus, dass situative Bedingungen den Geschlechtsunterschied erklären können. Danach verursachen die aktuellen Umstände, in denen Männer und Frauen leben, ein unterschiedliches Niveau im Politikinteresse. Nach diesem Erklärungsansatz haben Frauen durch Beanspruchung im Haushalt und in der Kinderbetreuung weniger Zeit, Energie und Gelegenheit sich um Politik zu kümmern (Westle/Schoen 2002: 216).

- Ein drittes Erklärungsmuster begründet das schwächere Interesse von Frauen mit den männlich geprägten Gesellschaftsstrukturen, etwa bei Ausbildungs- und Karrierewegen, die ihnen den Zugang zu 
politikbezogenen Sektoren und zu politisch relevanten Ressourcen erschweren. Auch führten die von Männern dominierten Gesellschaftsstrukturen zu einer mangelnden Berücksichtigung der Interessen von Frauen, was sich in ihrer stärkeren politischen Unzufriedenheit und einer größeren Politikverdrossenheit äußere (Westle/ Schoen 2002: 217, Neller 2002: 492).

- Als weitere Ursache für das stärkere Politikinteresse von Männern werden in der Literatur Effekte der soziale Erwünschtheit diskutiert. Dank der gesellschaftlichen Rollenvorstellungen ist demnach bei Männern die Neigung zur Übertreibung des politischen Interesses und Engagements stärker ausgeprägt als bei Frauen (vgl. van Deth 1990, Verba et al. 1997: 1054).

Darüber hinaus wird in der Literatur der steigende Anteil politisch Interessierter nicht selten mit modernisierungstheoretischen Aspekten in Zusammenhang gebracht: mit der Entwicklung und Ausweitung der Massenmedien, insbesondere mit dem Aufkommen des Fernsehens, dem Zuwachs an Freizeit sowie mit den Veränderungen in der Wertestruktur der Bevölkerung (Gabriel/van Deth 1995: 398f., Schmid 2004: 20).

\subsubsection{Individuelle Entwicklung und Stabilität des politischen Interesses im Lebensverlauf}

Politisches Interesse wurde lange Zeit als stabile persönliche Disposition betrachtet, die im Laufe der politischen Sozialisation entsteht und die als eine Art Persönlichkeitskonstante die individuelle politische Identität mitbestimmt (Gille 2000).

Inzwischen ist allerdings aus vielen empirischen Zeitreihenuntersuchungen bekannt, dass die Höhe des politischen Interesses in der Bevölkerung konjunkturellen Schwankungen unterliegt. Zum einen wird dies auf veränderte subjektive Aktivierungszustände zurückgeführt (vgl. Schneider 1995, Gille 2000). Infolge aktueller politischer Ereignisse - etwa durch 
Wahlen und den damit verbundenen Wahlkämpfen, spektakulären historischen Ereignissen wie Terroranschlägen, AKW-Unfällen oder in Deutschland der Wiedervereinigung - kommt es häufig zu einem Anstieg des Politikinteresses in der Bevölkerung (Periodeneffekte).

Aber auch biographische Phasen können die subjektiven Aktivierungszustände verändern und zu Variationen im Politikinteresse führen. Es wird beispielsweise angenommen, dass jüngere Menschen - u.a. aufgrund der noch geringen politischen Erfahrungen - grundsätzlich ein niedrigeres Niveau an Politikinteresse aufweisen und politischen Angelegenheiten im Vergleich zu anderen Lebensbereichen wie Ausbildung oder Partnerschaft eine nur geringe Bedeutung beimessen (vgl. van Deth 2000, Neller 2002b, Schmid 2004).

Dementsprechend konnte in verschiedenen Jugend-Längsschnittanalysen übereinstimmend festgestellt werden, dass sich das Interesse für Politik erst allmählich in der Jugend entwickelt und mit steigendem Alter zunimmt ${ }^{16}$ (vgl. etwa Fend 1991, Schmid 2004).

Hinweise auf einen lebenszyklischen Verlauf der Höhe des politischen Interesses geben auch Trendanalysen, welche die Entwicklung des Politikinteresses im Aggregat untersuchen. Danach folgt die Stärke des politischen Interesses meist einem kurvilinearen Verlauf mit Tiefpunkten zu Beginn und am Ende des Lebenszyklus und einer Hochphase im mittleren Erwachsenenalter (Vgl. Datenreport 2006, vgl. auch van Deth 2000: 51, Schmid 2004: 22$)^{17}$.

Für Aussagen zur langfristigen Entwicklung des politischen Interesses und ihrer Determinanten auf Individualebene sind jedoch Paneluntersuchungen

\footnotetext{
${ }^{16}$ Dieser Effekt wurde nicht nur für Deutschland ermittelt, sondern kann auch in international vergleichenden Studien für andere Länder gezeigt werden (vgl. Neller 2002b).

${ }^{17}$ Allerdings stellte van Deth fest, dass in einer Zeitreihe das Interesse der jüngeren Befragten in den 70er Jahren etwas über dem der älteren Befragten lag. Diesen Befund wertet er als Indiz dafür, dass die "normalen“ Lebenszykluseffekte durch starke politische Mobilisierungen (Generationeneffekt) wie in den 70er Jahren außer Kraft gesetzt werden können (vgl. van Deth: 51, Kaase 1989).
} 
notwendig, die einen langen Zeitraum - möglichst über mehrere Jahrzehnte hinweg - abdecken. Nur so können individuelle Entwicklungen in den verschiedenen Lebensphasen nachgezeichnet und eventuelle Zusammenhänge zwischen dem politischen Interesse und Einflüssen des Lebenszyklus aufgespürt werden ${ }^{18}$.

Solche Längsschnittuntersuchungen haben allerdings nach wie vor Seltenheitswert (vgl. Grob 2006: 65, Geißler 1996). Dieses Defizit ist in erster Linie im Mangel dauerhaft angelegter Panelstudien begründet, die sowohl eine Untersuchung früher Sozialisationseinflüsse sowie Aussagen zur langfristigen individuellen Entwicklung von politischen Orientierungen erlauben.

Eine der seltenen Längsschnittstudien zur langfristigen Untersuchung politischer Sozialisation ist die von Jennings und Niemi (1974, 1981, 1999) entwickelte Panelstudie, welche unter anderem auch die Untersuchung der langfristigen Stabilität des politischen Interesses ermöglicht.

Die im Jahr 1965 initiierte US-amerikanische Studie umfasst eine Stichrobe von 1669 ehemaligen High-School-Absolventen und deren Eltern, die bis heute insgesamt vier mal befragt wurden (1965, 1973, 1982, 1997) ${ }^{19}$.

In ihrer Analyse des politischen Interesses unterscheiden die Autoren zwischen solchen Befragten, die eine hohe politische Übereinstimmung mit ihren Eltern aufweisen (bezogen auf das Jahr 1965), und jenen, die in politischen Fragen nur wenig mit ihren Eltern gemein haben (J ennings/Stoker 1999: 38$)^{20}$.

Im Fall geringer politischer Übereinstimmung zwischen Eltern und Kindern ermittelten Jennings und Stoker (1999) moderate Stabilitätskoeffizienten für das politische Interesse (Pearsons $r$ ):

- 1965 - 1973: . 30 (Alter der Befragten 18 bis 26 Jahre)

- 1973 - 1982: .43 (Alter der Befragten 26 bis 35 Jahre)

\footnotetext{
18 Die Verwendung von Retrospektivbefragungen ist hier keine Lösung, da die retrospektive Messung von Einstellungen und Orientierungen als nicht valide gilt (vgl. Reimer 2001). Zur ebenfalls verwendeten Methode der Kohortenanalyse siehe Kapitel 6.

19 Die Eltern wurden nur zweimal wiederbefragt.

${ }^{20}$ Angaben zur Gesamtstabilität sind den Veröffentlichungen leider nicht zu entnehmen.
} 
- 1982 - 1997: .46 (Alter der Befragten 35 bis 50 Jahre)

War die politische Übereinstimmung zwischen Eltern und Kindern dagegen hoch, so lagen die Stabilitätskoeffizienten durchweg etwas höher, auch wenn die Unterschiede zwischen den beiden untersuchten Gruppen nicht signifikant sind $(p>=.05)$ :

- 1965 - 1973: .39 (Alter der Befragten 18 bis 26 Jahre)

- 1973 - 1982: .44 (Alter der Befragten 26 bis 35 Jahre)

- 1982 - 1997: .57 (Alter der Befragten 35 bis 50 Jahre)

Die Ergebnisse stützen insgesamt die These, wonach im späteren Lebensverlauf die Stabilität des Politikinteresses zunimmt: Obwohl die Befragungsspanne im fortgeschrittenen Erwachsenenalter (1982 bis 1997) fünfzehn Jahre beträgt, liegen die Koeffizienten (.46/.57) in beiden Gruppen der untersuchten Kohorte deutlich über den Stabilitätskoeffizienten der jüngeren Erwachsenen (.30/.39).

Zur langfristigen Entwicklung des konventionellen politischen Interesses in Deutschland liegt eine vor kurzem erschienene Untersuchung mit den Daten der Follow-Up- Befragung der von Fend geleiteten LIFE- Studie vor. ${ }^{21}$ In dieser Untersuchung konnte Grob (2006) einen nicht unerheblichen Einfluss der Jugendphase auf die langfristige Entwicklung des Politikinteresses ausfindig machen. Danach liegt die relative Stabilität des Politikinteresses zwischen dem Jugendalter (16 Jahre) und dem 35. Lebensjahr bei Beta $=.54$ und erreicht damit einen erklärten Varianzanteil von 28,6\% (Grob 2006: 82).

\footnotetext{
${ }^{21}$ Bei der LIFE-Studie handelt es sich um eine von den Universitäten Konstanz und Zürich unter der Leitung von Helmut Fend durchgeführte Langzeitstudie, die die biographische Entwicklung von etwa 1500 Jugendlichen vom 12. bis zum 35 Lebensjahr in drei Befragungswellen untersucht $(1979,1983,1997)$ und auch Untersuchungen zur politischen Sozialisation erlaubt (vgl. Zeitschrift für Soziologie der Erziehung und Sozialisation, Heft 1/2005 (ZSE) mit dem Themenschwerpunkt, LifE-Studie'). Diese Daten sind allerdings von den Primärforschern bisher nicht für die wissenschaftliche Forschung freigegeben. Für die USA ist v.a. die weiter oben beschriebene Studie von Jennings und Niemi zu erwähnen.
} 
Zudem konnte Grob die Hypothese vom Anstieg des Politikinteresses im Verlauf der Jugendphase bestätigen: in der untersuchten Alterskohorte nahm zwischen dem 12. (bei Jungen, bei Mädchen ab dem 13. Lebensjahr) und dem 16. Lebensjahr das Politikinteresse der befragten Jugendlichen kontinuierlich zu (Grob 2006: 74).

Zur Erklärung des späteren Politikinteresses untersuchte Grob manifeste Sozialisationseinflusse während der Adoleszenz in Familie und Schule. Dabei konnte er feststellen, dass der Einfluss des elterlichen Politikinteresses für jenes der Nachkommen im Erwachsenenalter zwar substanziell, in der Effektstärke aber begrenzt ist (Grob 2006: 89).

In diesem Zusammenhang spielt das Geschlecht der Befragten eine bedeutsame Rolle: für die Höhe des Politikinteresses der Frauen ist der langfristige Einfluss des elterlichen Politikinteresses wesentlich wichtiger als für Männer. Das männliche Politikinteresse wird dagegen recht deutlich von der sozialen Lage im Elternhauses beeinflusst (Grob 2006: 86) - und dies auch noch im Alter von 35 Jahren.

Der Einfluss schulischer Kontextmerkmale auf die Entwicklung von politischem Interesse ist Grobs Untersuchungen zufolge als erstaunlich gering einzustufen (2006: 88f.). Zwar ist der höchste Schulabschluss ein relevanter Prädiktor für das politische Interesse im Erwachsenenalter. Andere schulische Merkmale - wie etwa der besuchte Schultyp - spielen jedoch nur eine sehr geringe Rolle.

\subsection{Gesellschaftspolitische Werte}

Mit Kluckhohn (1951) wird in der empirischen Werteforschung unter einem Wert meist eine abstrakte "Konzeption des Wünschenswerten“ verstanden (vgl. Maag 1991: 21f.).

Gesellschaftspolitische Werte sind demzufolge normative Maßstäbe, nach der die gesellschaftlichen Zustände beurteilt werden können. Sie sind kulturelle Bestandteile eines gesellschaftlichen Systems und können sich zwischen verschiedenen politischen Systemen erheblich unterscheiden (vgl. Arzheimer 2005: 285). Durch den Prozess der Internalisierung werden Werte im Verlauf 
der Sozialisation in das individuelle Überzeugungssystem integriert und nehmen dort als Wertorientierungen ${ }^{22}$ eine herausgehobene Stellung ein.

Für die Sozialwissenschaften sind Werte bzw. Wertorientierungen vor allem wegen zweier Eigenschaften von großem Interesse (vgl. Arzheimer 2005: 286):

Zum einen gelten Wertorientierungen als sehr veränderungsresistent und persönlichkeitsstrukturell relativ stabil verankert. Sind sie erst einmal im individuellen Überzeugungssysteme integriert, so die häufig geäußerte Auffassung, dann bleiben Wertorientierungen unter normalen Umständen ein Leben lang stabil (vgl. Maag 1991).

Zum anderen nehmen Wertorientierungen im individuellen Überzeugungsgefüge eine zentrale und übergeordnete Stellung ein. In dieser Position steuern sie als Maßstäbe mit einem höheren Allgemeinheitsgrad die Bewertung untergeordneter Einstellungsobjekte und Handlungsalternativen (van Deth/Scarbrough 1995). Damit strukturieren Wertorientierungen das individuelle Einstellungsgefüge und beeinflussen die individuellen Verhaltensstile (Bürklin/Klein 1998: 139, vgl. Arzheimer 2005: 286).

Verändert sich in einer Gesellschaft die Verteilung der Wertorientierungen merklich, dann kann von einem gesellschaftlichen Wertewandel gesprochen werden. Die Frage nach den Ursachen eines solchen Wertewandels wird in der Wissenschaft seit langem kontrovers diskutiert und führte zu verschiedenen Theorien, die den Wertewandel zumeist entweder auf gesellschaftliche Modernisierungsprozesse zurückführen oder aber von zyklischen Veränderungen ausgehen (vgl. für einen Überblick Bürklin 1988, Maag 1991).

\footnotetext{
${ }^{22}$ In den am methodologischen Individualismus ausgerichteten Sozialwissenschaften wird in der Regel unterschieden zwischen Werten als kulturellen Objekten und individuellen Wertorientierungen, d.h. den individuellen Einstellungen gegenüber jenen Werten (Arzheimer 2005).
} 


\subsubsection{Die These vom postmaterialistischen Wertewandel nach I nglehart}

Die wohl prominenteste Theorie des Wertewandels ist die in den 60er Jahren von Ronald Inglehart entwickelte These vom postmaterialistischen Wertewandel (Inglehart 1977, 1989, Jagodzinski 1985: 333).

In seiner Theorie geht Inglehart von einem eindimensionalen Wertewandel in den westlichen Gesellschaften nach dem zweiten Weltkrieg aus. Dieser Wertewandel besteht seiner Beobachtung nach in der Verdrängung materieller Wertpräferenzen zugunsten postmaterieller Wertorientierungen.

Unter materialistischen Werten versteht Inglehart das Streben nach wirtschaftlichem Wohlstand und dessen Absicherung gegen innere und äußere Bedrohungen. Postmaterialistische Wertorientierung bedeutet dagegen für inn eine Höherbewertung immaterieller Aspekte wie beispielsweise dem Wunsch nach umfassenden Selbstentfaltungs- und verwirklichungsmöglichkeiten, nach politischer Partizipation oder Gleichberechtigung der Geschlechter (Inglehart 1979: 279).

Zur Erklärung dieses Wertewandels formulierte Inglehart zwei ,Schlüsselhypothesen': Die Mangel- und die Sozialisationsthese.

Der Mangelhypothese liegt in Anlehnung an die Motivationstheorie von Maslow (1954) die Vorstellung zugrunde, dass Menschen eine bestimmte Anzahl von hierarchisch angeordneten Grundbedürfnissen besitzen, denen umso mehr Aufmerksamkeit geschenkt wird, je weniger ihre Befriedigung gewährleistet ist (,Gesetz vom abnehmenden Grenznutzen' vgl. Inglehart 1989: 92f.).

I hre besondere Bedeutung gewinnt die Mangelhypothese jedoch durch die Verbindung mit der Sozialisationshypothese. Danach werden individuelle Wertorientierungen während der ,formativen' Phase im J ugendalter erworben und bleiben im Laufe des Lebens gegenüber Veränderungen weitgehend stabil. Zwar betrachtet Inglehart Wertorientierungen als nicht vollkommen wandlungsresistent, ist aber das Erwachsenenalter erst einmal erreicht, so werden grundsätzliche individuellen Werteveränderungen sehr unwahrscheinlich (Inglehart 1989: 70). Die Entwicklung der individuellen Wertorientierungen vollzieht sich somit nicht primär im Kontext mit aktuellen 
Lebensbedingungen, sondern spiegeln die Verhältnisse wieder, unter denen ein Mensch während seiner ,formativen Phase' aufgewachsen ist. Dabei sind in erster Linie die materiellen Lebensumstände der frühen Sozialisationsphase von Belang: "Die Generation, die nach dem Zweiten Weltkrieg geboren wurde und in einer Zeit noch nie da gewesenen Wohlstands aufwuchs, neigt wahrscheinlich dazu, nicht-materiellen Zielen eine relativ hohe Priorität einzuräumen. Ihre Eltern und Großeltern dagegen, die während der prägenden Jahre Hunger und politische Instabilität erfuhren, werden ökonomische und physische Sicherheit höher einschätzen" (Inglehart 1979: 280).

Der auf der Aggregatebene konstatierte Wertewandel - hin zu mehr postmaterialistischen Werten - wird, so die These, durch das sukzessive Nachwachsen der in Wohlstand aufgewachsenen jüngeren Generationen verursacht. Diese ersetzen im Laufe der Zeit die älteren Generationen mit ihren überwiegend materialistischen Wertemustern (Generationeneffekte, vgl. Bürklin/ Klein 1998: 147). Diese Beobachtung führte Inglehart folgerichtig zu der Prognose, dass in den 90er Jahren die Mehrheit der Bevölkerung in westlichen Industriegesellschaften über postmaterialistische Wertemuster verfügen müsse (vgl. Inglehart 1971: 991).

Ingleharts Thesen sind erheblicher inhaltlicher wie methodischer Kritik ausgesetzt. Die bis heute anhaltende Kritik kann hier allerdings nur ansatzweise wiedergegeben werden (vgl. Bürklin et al. 1996).

Ein inhaltlicher Kritikpunkt entzündet sich z.B. an Ingleharts Annahme, dass die persönlichen Wertepräferenzen nach der primären Sozialisation im wesentlichen stabil bleiben und sich kaum mehr verändern.

Da politische Sozialisation als lebenslanger Prozess zu verstehen sei, müsse so einige der Kritiker - von einer intra-individuellen Wandelbarkeit der Wertorientierungen auch im späteren Lebensverlauf ausgegangen werden (vgl. Bürklin/ Klein 1998: 144, vgl. auch Kapitel 2). Neben den von Inglehart beschriebenen Sozialisations- oder Generationeneffekten kämen daher auch lebenszyklische und periodische Wandlungsmuster als Ursache für Veränderungen innerhalb der gesellschaftlichen Wertestruktur in Frage (Bürklin et al 1994: 583f.). 
Der Lebenszyklus-Hypothese zufolge wandeln sich Wertorientierungen mit individuellen Veränderungen der Lebenslage und veränderten Interessen im Lebensverlauf (vgl auch Glenn und Hefner 1972). Illustrative Beispiele für solche Lebenszyklen sind z. B. Berufstätigkeitsphasen, Partner- oder Elternschaft.

Verändern sich also gesellschaftliche Wertorientierungen im Zusammenhang mit solchen Lebensabschnitten unabhängig von der Zugehörigkeit zu einer Kohorte, so deutet dies auf einen Lebenszykluseffekt hin (Abramson 1979).

Für das Makrosystem bedeuten lebenszyklische Wertewandelprozesse eine relative Stabilität, da sich die Träger neuer Werte durch die LebensverlaufsEffekte immer wieder in das bestehende System integrieren. Gravierende Veränderungen der gesellschaftlichen Wertestruktur sind hier nur zu erwarten, wenn sich die demographische Altersstruktur oder die relative Dauer einzelner Lebenszyklusphasen wandelt (vgl. Bürklin 1994: 584).

Darüber hinaus gibt es auch allgemeine Lebensbedingungen, die zu einem Wandel der Wertorientierungen aller Individuen in einer Gesellschaft führen können, unabhängig von der Generationszugehörigkeit oder dem Lebenszyklus des einzelnen (Flanagan 1979: 260). So ist z.B. denkbar, dass postmaterialistische Wertorientierungen in wirtschaftlich prosperierenden Phasen bei Menschen aller Generationen oder Lebensphasen an Zulauf gewinnen, während in Zeiten ökonomischer Rezession sicherheitsorientierte Werte im Vordergrund stehen (Periodeneffekt).

Anders als die Generationen-Hypothese geht diese Sichtweise von einer schwächeren intra-individuellen Wertestabilität aus und unterstellt eine stärkere Sensibilität der Individuen für Zeitgeisteinflüsse. Auf der gesellschaftlichen Makroebene führen Periodeneffekte daher auch zu grundsätzlich rascheren Veränderungen der Wertestruktur (Bürklin 1994: 584).

Neben diesen generellen Kritikpunkten hinsichtlich der Wandelbarkeit von Wertorientierungen wurden viele weitere inhaltliche Einwände gegen die Inglehartsche Theorie vorgebracht. Kritisiert wurde z.B. das von Inglehart unterstellte bipolare Wertekontinuum, welches das Spektrum 
gesellschaftlicher Werte auf zwei generalisierte Wertetypen reduziert, sowie die mangelnde Abgrenzung zwischen Werten und Bedürfnissen (etwa van Deth 1983, Herz 1979, 1987, Flanagan 1982, Bürklin et al. 1994, vgl. für einen Überblick Maag 1991: 56f, Bürklin/Klein 1998: 148f.).

Ein weiterer, häufig bemängelter Aspekt betrifft die Reliabilität und Validität des Messinstrumentes, des sog. Inglehart-Indexes ${ }^{23}$.

So zeigen Aggregatuntersuchungen, dass der Anteil der Materialisten in der Bevölkerung zunimmt, wenn die Inflationsrate in einem Land ansteigt (vgl. etwa Gabriel 1986, Klein 1995, Jagodzinski 1996). Da Werte allerdings als langfristig relativ stabile intra-individuelle Orientierungen konzeptioniert sind, wird dieser Befund als Hinweis darauf gewertet, dass mit dem InglehartIndex eher kurzfristig veränderbare Einstellungen als tief verwurzelte Wertorientierungen gemessen werden (vgl. Gabriel 1986, Bürklin/Klein 1998: 148, Klein 1995: 218).

\subsubsection{Empirische Befunde zur Postmaterialis- musthese}

Ungeachtet der Kritik an Ingleharts Thesen und seinem Messinstrument ist der von Inglehart entwickelte Postmaterialismus-Index bis heute das Standard-Instrument zur Messung gesellschaftlicher Wertorientierung. Dies gilt sowohl für sozialwissenschaftliche Studien in der $\mathrm{BRD}^{24}$, als auch für international vergleichenden Untersuchungen wie dem Eurobarometer, den ,Political Action'- oder ,World Value'- Studies.

Für die alte Bundesrepublik ${ }^{25}$ zeigen Zeitreihenuntersuchungen seit den 70er Jahren des letzten Jahrhunderts im Bevölkerungsdurchschnitt zwar eine

\footnotetext{
${ }^{23}$ Zum Messinstrument des Inglehart-Indexes siehe Kapitel 6.

24 Etwa in den Allgemeinen Bevölkerungsumfragen Sozialwissenschaften (ALLBUS), in deutschen Wahlstudien oder dem Sozio-ökonomischen Panel (SOEP).

${ }^{25}$ Für die ostdeutschen Länder liegen naturgemäß nur sehr kurze Zeitreihen vor. Sie zeigen jedoch, dass die ostdeutschen Befragten, etwas stärker den materialistischen Politikzielen zuneigen (vgl. Arzheimer 2005b: 302).
} 
abnehmende Unterstützung rein materialistischer Wertvorstellungen, der Anteil der reinen Postmaterialisten stieg jedoch entgegen Ingleharts Prognose nur sehr moderat an (Bürklin/Klein 1998, Klein 2005, Arzheimer 2005).

Stark erhöht hat sich dagegen der Anteil der sogenannten Mischtypen, die weder dem materialistischen- noch dem postmaterialistischen Wertetyp eindeutig zugeordnet werden können.

Dieser Befund wird von einigen Autoren als Hinweis darauf gewertet, dass der Wertewandel nicht in Richtung des reinen Postmaterialismus, sondern vielmehr hin zu einer Synthese aus materialistischen und postmaterialistischen Werten verläuft (Bürklin/Klein 1998: 151, Klein 2005a: 439).

Überdies belegen die genannten Studien für die BRD, wie auch internationale Untersuchungen (Clarke et al. 1999, vgl. auch Maag 1991: 55), kurzfristige Schwankungen bei den Inglehartschen Wertorientierungen, die von Kritikern auf die Veränderungen der wirtschaftlichen Rahmenbedingungen wie Inflationsrate oder Arbeitslosenquote zurückgeführt werden (Gabriel 1986, Klein 1995, Klein/Pötschke 2000).

Neben diesen Periodeneffekten infolge wirtschaftlicher Entwicklungen offenbaren weitere Analysen auch eine Korrespondenz der Wertorientierungen mit den Bedingungen der aktuellen individuellen Lebenssituation. Bürklin et al. (1994) zeigen beispielsweise auf, dass für die Aggregatveränderung sogenannter aquisitiver und traditioneller Werte $^{26}$ in erster Linie Lebenszyklus-Effekte verantwortlich sind (Bürklin et al. 1994: 599).

\subsubsection{Individuelle Entwicklung und Determinanten der politischen Wertorientierungen im Lebensverlauf}

In Ermangelung dauerhaft angelegter Panelstudien, die eine Untersuchung früher Sozialisationseinflüsse sowie Aussagen zur langfristigen individuellen Werteentwicklung zulassen, wurden die vorliegenden empirischen

${ }^{26}$ Die Messung von Wertorientierung basieren auf allerdings nicht auf dem von Inglehart entwickelten Messinstrument, sondern auf einem Ratingverfahren. 
Untersuchungen zum Wertewandel zumeist auf Basis von Trenddaten durchgeführt (vgl. z. B. Inglehart 1981, Jagodzinski 1983, Mnich 1989, Bürklin et al. 1994). Bei diesen Untersuchungen handelt es sich größtenteils um Kohortenanalysen, die allerdings mit einer Reihe methodischer Probleme behaftet sind und keine Aussagen zur intra-individuellen Wertestabilität zulassen (zur Kohortenanalyse siehe Kapitel 6).

Analysen, die auf Panelstudien mit kurzen bzw. mittelangen Untersuchungszeiträumen in der Bundesrepublik basieren, zeigen bei Verwendung des Inglehart-Indexes eine unterschiedlich starke intraindividuelle Stabilität gesellschaftlicher Wertorientierungen.

So ermittelte Dalton (1981) zwischen 1976 und 1979 in einer Panelstudie mit Jugendlichen und jungen Erwachsenen etwa 70\% Befragte mit gleichen Wertepräferenzen ${ }^{27}$. Und auch Inglehart (1989) und de Graaf et al. (1989) fanden in der Bundesrepublik - berechnet auf der Grundlage der Political Action Studies 1974 bis 1980 - mit einem Koeffizienten von Beta=.72 bzw. Beta $=.65$ recht hohe Stabilitätswerte ${ }^{28}$.

In jüngeren Untersuchungen zur individuellen Wertestabilität kommen Arzheimer/Klein (2000) und Klein/Pötschke (2004) zu ganz anderen Resultaten.

Auf der Grundlage eines Wahl-Panels (vgl. Falter et al. 2000) stellen Arzheimer und Klein (2000: 393) für Ost- wie für Westdeutschland fest, dass die im Jahr 1998 mit dem Inglehart-Index gemessenen Wertorientierungen in einem „nur schwachen Zusammenhang mit den Messwerten aus dem Jahr

\footnotetext{
${ }^{27}$ Die Analyse von Dalton wurde jedoch von Jagodzinski (1984) methodisch scharf kritisiert. In einer Reanalyse konnte Jagodzinski die von Dalton berechneten Stabilitätskoeffizienten nicht bestätigen. $\mathrm{Er}$ ermittelte vielmehr erheblich niedrigere Stabilitätskoeffizienten für postmaterialistische Werte.

${ }^{28}$ Allerdings benutzten de Graaf et al. (1989) und Inglehart (1989) für ihrer Analyse nicht das Standard-Messinstrument mit vier Items (vgl. Kapitel 6). Sie verwendeten stattdessen eine konfirmatorische Fakorenanalyse, in der sie neben dem vier-Item- Standard-Instrument auch ein zweites, längeres Ranking-Instrument mit acht Items benutzten.
} 
1994 stehen“, was mit dem Inglehartschen Konzept langfristiger Wertorientierungen nicht vereinbar ist.

Und auch eine von Klein und Pötschke veröffentlichte Analyse auf Basis des Sozioökonomischen Panels (SOEP) über einen Zeitraum von immerhin 12 Jahren kommt zu einem ähnlichen Ergebnis. Ihre Befunde bestätigen zwar Ingleharts Erwartungen zum Teil - so fanden sie z.B. signifikante Generationeneffekte - insgesamt aber zeigte sich ein eher geringes Maß an intra-individueller Stabilität der gesellschaftlichen Wertorientierungen (Klein/ Pötschke 2004: 445). Dabei ermittelten die Autoren insbesondere für die beiden jüngsten Generationen sowie für die Hochgebildeten Lebenszykluseffekte, die der Postmaterialismus-These entgegen stehen (Klein/ Pötschke 2004: 453) ${ }^{29}$.

Außerdem konnte in verschiedenen Untersuchungen gezeigt werden, dass die Stabilität der politischen Wertorientierungen abhängig ist von der Höhe des Politikinteresses: Politisch stark Interessierte verfügen danach über deutlich stabilere Wertorientierungen als solche, die für politische Angelegenheiten nur wenig Interesse aufbringen (vgl. Kapitel 4.1, van Deth 1990, Maag 1991).

Aufgrund dieser Befunde ist es durchaus plausibel, die Gültigkeit der Inglehartschen Thesen infrage zu stellen - immerhin ist die Stabilität der Werte eine wesentliche Voraussetzung für die Existenz der von Inglehart postulierten formativen Phase (vgl. Arzheimer 2005, siehe auch Kapitel 5).

${ }^{29}$ Auch Meulemann und Birkelbach kommen in einer Panelanalyse zu dem Schluss, dass biographische Erfahrungen der Berufstätigkeitsdauer gewichtiger sind für die Ausprägung der von innen untersuchten Wertorientierungen als Erfahrungen, die in der ,formativen Phase' gesammelt wurden. Allerdings schöpfen die Autoren nicht alle Möglichkeiten ihres Datensatzes zur Messung früher Sozialisationseinflüsse aus (siehe Kapitel 6).

Des weiteren verwenden Meulemann und Birkelbach (2001) zur Messung der Wertorientierungen nicht den von Inglehart entwickelten Index, sondern ein von Meulemann et al. (1987) entworfenes Instrument, mit dem die Annerkennung von Sachzwängen (damit sind die "materialistischen“ Bestandteile des Inglehart-Indexes gemeint) unabhängig von den Wertansprüchen (hiermit sind die „postmaterialistischen“ Bestandteile des Inglehart-Indexes gemeint) gemessen werden sollen. 


\subsection{Zusammenfassung}

Fasst man die vorliegenden empirischen Ergebnisse zusammen, so zeigt sich ein differenziertes Bild: Zwar sind bei der Ausbildung des Politikinteresses und der Entwicklung der Wertorientierungen in gewissem Maße Perioden- und Lebenszykluseffekte sichtbar, aber es finden sich auch langfristige Sozialisationswirkungen bzw. auf solche hinweisende Generationeneffekte.

Im Hinblick auf Wertorientierungen lässt sich festhalten, dass es zwar Hinweise auf langfristig wirksame Sozialisationseffekte der Jugend gibt, aber nur unzureichende Befunde zu den Inhalten der zugrundeliegenden Sozialisationserfahrungen vorliegen.

Darüber hinaus existieren bis heute keine empirischen Erkenntnisse über die Relevanz latenter Sozialisationseinflüsse der Jugendphase auf gesellschaftspolitische Werte im Erwachsenenalter. Auch über die Bedeutung latenter jugendlicher Sozialisationserfahrungen für das Politikinteresse im Erwachsenenalter - beispielsweise in Familie oder Schule - ist bislang nichts bekannt. 


\section{$5 \quad$ Hypothesen}

Für die Untersuchung der eingangs erläuterten Forschungsfragen werden nun - vor dem Hintergrund des skizzierten theoretischen Bezugsrahmens sowie der empirischen Befunde - überprüfbare Hypothesen formuliert. Die Hypothesen zum Politikinteresse und zu den politischen Werten werden dabei in getrennten Abschnitten dargestellt und kurz erläutert.

\subsection{Hypothesen zum politischen Interesse}

\section{Hypothesen zur Stabilität des politischen I nteresses}

Wie oben ausgeführt ist die Vorraussetzung für langfristig wirksame Sozialisationsprägungen ein Mindestmaß an Stabilität des politischen Interesses. Andererseits muss berücksichtigt werden, dass zwischen den beiden Untersuchungszeitpunkten 1984/85 und 1996/97 ein sehr langer Zeitraum von 13 Jahren besteht, wodurch die Stabilität tendenziell reduziert wird:

Hypothese A1: Die Stabilität des politischen Interesses im Erwachsenalter zwischen dem 30. und 43. Lebensjahr liegt mindestens im mittleren Bereich (Korrelationskoeffizient $>0.4$ ).

\section{Zum Einfluss von Ressourcenausstattung und soziodemographischen Faktoren auf das politische I nteresse im 30. Lebensjahr}

Der folgenden Hypothese liegt die Vorstellung zugrunde, dass in sozioökonomisch privilegierten Elternhäusern den Jugendlichen mehr Kenntnisse und Fähigkeiten vermittelt werden, die für die Entwicklung von politischem Interesse relevant sind. Diese Lernprozesse beziehen sich zum einen auf vorpolitische Einstellungen und Dispositionen wie Kompetenz- und Effizienzgefühle. Zum anderen verfügen in sozial privilegierten und ,bildungsnahen' Schichten die Eltern selbst über ein höheres Politikinteresse (siehe Kapitel 4, vgl. auch Milbrath/Goel 1977, Verba et al. 1995). Über 
Transmissions- bzw. Kommunikationsprozesse sollte ein höheres elterliches Interesse an Politik die Entwicklung des Politikinteresses der Nachkommen positiv beeinflussen.

Hypothese $\mathrm{AFI}^{30}$ : Je höher der sozio-ökonomische Status des Elternhauses während der Jugend ist, desto höher ist das politische Interesse im 30. Lebensjahr.

Kognitiv kompetente und gut ausgebildete Menschen verfügen über intellektuelle Mittel, die es innen erleichtern komplexe politische Zusammenhänge zu verstehen und einzuordnen. Sie verfügen folglich auch über eine bessere Orientierung im politischen Raum und können die Bedeutung der Politik für das eigene Leben genauer einschätzen. Diese Kompetenzen sollten das politische Interesse und die Partizipationsbereitschaft fördern:

Hypothese AF2: Je höher die Ausprägung der kognitiven Kompetenz der Schüler und Schülerinnen, desto höher ist das politische Interesse im 30. Lebensjahr.

$\mathrm{Da}$ trotz des Anstiegs des weiblichen Bildungsniveaus ein Geschlechtsunterschied in der Höhe des Politikinteresses fortbesteht (zur Bildungshypothese siehe AS1 vgl. Schoen/Westle 2001), ist zu erwarten, dass sich auch in der hier vorliegenden bildungsprivilegierten Untersuchungsstichprobe Frauen weniger politikinteressiert äußern als Männer.

Hypothese AF3: Frauen sollten ein geringeres politisches Interesse als Männer aufweisen.

\footnotetext{
${ }^{30}$ Die Hypothesennummerierung ist folgendermaßen zu verstehen:

AF: Hypothesen zur ,frühen' Sozialisation

AS: Hypothesen zur ,späten' Sozialisation

Al: Hypothesen zu Interaktionseffekten.

Entsprechende Nummerierungen für den Hypothesenblock B.
} 


\section{Zum Einfluss latenter Sozialisationserfahrungen in der J ugend}

Ein vertrauensvolles und unterstützendes Klima im Elternhaus und eine Erziehung zur Selbstständigkeit fördert die Entwicklung „vorpolitischer“ Persönlichkeitsmerkmale und Orientierungen wie Vertrauen, Selbstwert- bzw. Kompetenzgefühl oder Responsivitätsempfinden. Diese vorpolitische Eigenschaften stehen in deutlichem Zusammenhang mit politischem Interesse, politischer Effektivität und politischer Aktivitätsbereitschaft.

Hypothese AF4: Je positiver die Jugendlichen das Familienklima wahrnehmen, desto höher ist das politische Interesse im 30. Lebensjahr.

Hypothese AF5: Je mehr Wert die Eltern auf die Erziehung zu selbständigem Denken und Handeln legen, desto höher ist das politische Interesse im 30. Lebensjahr.

Auch außerfamiliale latente Sozialisationserfahrungen sollten Einfluss auf das politische Interesse haben: Die im Schulalltag stattfindenden Auseinandersetzungen mit Autoritäten dürften das Bewusstsein für gesellschaftliche Machtstrukturen schärfen. Das Verständnis und das Interesse für politische Angelegenheiten sollte dadurch gefördert werden:

Hypothese AF6: Je umfangreicher die Auseinandersetzungen mit Autoritäten im Schulkontext sind, desto höher ist das politische Interesse im 30. Lebensjahr.

\section{Zum Einfluss manifester Sozialisationserfahrungen in der J ugend}

Durch die Beschäftigung mit gesellschaftlichen und politischen Themen im Schulunterricht wird politisches Wissen und das Verständnis in komplexe politische Zusammenhänge vermittelt. Darüber hinaus werden im Politikoder Sozialkundeunterricht politische Kommunikations- und Argumentationsfähigkeiten gefördert, was die Entwicklung von Politikinteresse und politischen Kompetenzgefühlen begünstigt. 
Hypothese AF7: Je mehr die Schülerinnen und Schüler im Unterricht mit gesellschaftlichen Themen in Kontakt kommen, desto höher ist das politische Interesse im 30. Lebensjahr.

Wird politische Partizipation in der Jugend aktiv erlebt, positiv bewertet oder im sozialen Umfeld mit hohem Ansehen belohnt, so wirkt dies ebenfalls politisch aktivierend:

Hypothese AF8: Je mehr politische Partizipationserfahrungen in der Jugendzeit gesammelt werden, desto höher ist das politische Interesse im 30 . Lebensjahr.

Hypothese AF9: Je positiver die Einstellung zur politischen Partizipation in der Jugend, desto höher ist das politische Interesse im 30. Lebensjahr.

Hypothese AF10: Je positiver das perzipierte Image politischer Partizipation in der Schulklasse, desto höher ist das politische Interesse im 30. Lebensjahr.

\section{Zum Einfluss sekundärer Sozialisationserfahrungen}

Neben langfristig wirksamen Sozialisationseffekten aus der Jugendzeit sind auch Einflüsse des späteren Erwachsenenalters zu erwarten. Wenn die ,sensible' Phase der politischen Sozialisation tatsächlich in der Zeit der mittleren Adoleszenz liegt, dann sollten die Effekte der sekundären Sozialisation allerdings geringer ausfallen als jene der Jugendzeit.

Zunächst einmal sollte die Höhe des erlangten Bildungsniveaus ${ }^{31}$ die Stärke des politischen Interesses beeinflussen. Die Bildung sollte in diesem Kontext über zwei Mechanismen wirksam sein: Zum einen sollten mit steigen Bildung wichtige Fähigkeiten wie intellektuelle oder sprachliche Kompetenzen

\footnotetext{
${ }^{31}$ Die Höhe des erworbenen Bildungszertifikats reflektiert zwar zu einem Großteil die sozioökonomischen Ressourcen und Bedingungen der frühen Sozialisationsphase, da aber die meisten Befragten in der hier vorliegenden Stichprobe ihren höchsten Bildungsabschluss erst nach der mittleren Adoleszenz erreichten, wird die Hypothese zum Bildungsniveau unter der Rubrik der späteren Sozialisationserfahrungen aufgeführt.
} 
zunehmen (vgl. Hypothese AF2). Zum anderen dürfte ein längeres Verbleiben in Bildungsinstitutionen die Auseinandersetzung mit - und damit das Interesse an - politischen Gegenständen fördern (Bildungsmoratorium) ${ }^{32}$.

Hypothese AS1: Je höher der erreichte Bildungsabschluss, desto höher ist das politische Interesse im 30. Lebensjahr.

Neben der Bildung sollte auch die aktuelle sozio-ökonomische Lage einen Einfluss auf die Höhe des Politikinteresses haben:

Hypothese AS2: Je höher die gegenwärtige individuelle sozio-ökonomische Stellung, desto höher ist das politische Interesse.

Eine besondere Beanspruchung durch die Betreuung von Kindern sollte dagegen weniger Zeit und Energie für Interesse an politischen Angelegenheiten lassen (vgl. Kapitel 4):

Hypothese AS4: Je größer die Beanspruchung durch die Betreuung von Kindern, desto geringer ist das politische Interesse im Erwachsenalter.

$\mathrm{Zu}$ den relevanten sekundären Sozialisationserfahrungen gehören außer situativen Faktoren der aktuellen Lebensumstände auch politische Partizipationserfahrungen:

Hypothese AS5: Je mehr politische Partizipationserfahrungen nach der Jugendzeit gesammelt werden, desto höher ist das politische Interesse im 30. Lebensjahr.

Da grundsätzlich von einer geschlechtspezifischen Sozialisation auszugehen ist, wird zusätzlich vermutet, dass zwischen Männern und Frauen Unterschiede bestehen:

\footnotetext{
${ }^{32}$ Allerdings ist in der sehr selektiven Stichprobe ehemaliger Gymnasiasten die Variation der Bildungsabschlüsse nicht sehr groß, so dass die Effekte eher gering ausgeprägt sein dürften.
} 
Hypothese AG1: Die Erklärungsstruktur- und Kraft der Erklärungsfaktoren für das politische Interesse weist geschlechtsspezifische Unterschiede auf.

\section{Interaktionseffekte zur Stabilität des Politikinteresses}

Aus der Literatur ist bekannt, dass bei der Stabilität politischer Dispositionen und Orientierungen Interaktionseffekte vorliegen. So wächst mit der Höhe des Politikinteresses sowie des Bildungsniveaus die Fähigkeit zur Ausbildung eines stabilen politischen Orientierungssystems (vgl. Converse 1964) und damit auch die Stabilität des politischen Interesses. Folgende Interaktionseffekte können daher erwartet werden:

Hypothese Al1: Bei starkem Politikinteresse ist seine Stabilität bei gleichzeitiger Berücksichtigung der erklärenden Variablen größer, als bei schwachem Politikinteresse.

Hypothese Al2: Die Stabilität des politischen Interesses steigt bei gleichzeitiger Berücksichtigung der erklärenden Variablen mit zunehmender Bildung an. 


\subsection{Hypothesen zu Wertorientierungen}

\section{Hypothese zur Stabilität der Wertorientierungen}

Die Hypothese zur Stabilität der Wertorientierungen entspricht der Erwartung hinsichtlich des politischen Interesses:

Hypothese B1: Die Stabilität der Wertorientierungen im Erwachsenalter zwischen dem 30. und 43. Lebensjahr liegt mindestens im mittleren Bereich (Korrelationskoeffizient $>0.4$ ).

\section{Zum Einfluss von Ressourcenausstattung und soziodemographischen Faktoren auf Wertorientierungen im 30. Lebensjahr}

Die entscheidende Ursache für die Ausbildung postmaterialistischer Wertorientierungen sieht Inglehart in der Sicherheit der materiellen Lebensumstände während der frühen Sozialisationsphase:

Hypothese BF1: Je höher der sozio-ökonomische Status des Elternhauses während der Jugend, desto postmaterialistischer sind die Wertorientierungen im 30. Lebensjahr.

Da kognitive Fähigkeiten $z u$ höheren Bildungsabschlüssen und höhere Bildungszertifikate zu einer höheren sozio-ökonomischen Stellung führen sollte, lässt sich folgende Erwartung formulieren:

Hypothese BF2: Je höher die kognitive Kompetenz der Schüler und Schülerinnen ausgeprägt ist, desto postmaterialistischer sind die Wertorientierungen im 30. Lebensjahr.

\section{Zum Einfluss latenter Sozialisationserfahrungen in der J ugend}

Postmaterialistische Wertorientierungen im Inglehartschen Sinne umfassen eine Höherbewertung der Meinungsfreiheit und die Forderung nach Ausweitung politischer Teilhabe. Werden Jugendliche von ihren Eltern zur 
Selbstständig- und Kritikfähigkeit erzogen, so sollten sie vermehrt solche postmaterialistische Ansprüche stellen.

Hypothese BF4: Je mehr Wert Eltern auf die Erziehung zu selbständigem Denken und Handeln legen, desto postmaterialistischer sind die Wertorientierungen im 30. Lebensjahr.

Postmaterialistische Ansprüche sollten auch befördert werden, wenn häufig Auseinandersetzungen mit schulischen Autoritäten stattfinden. Wer im Schulalltag oft Konfrontationen mit Lehrern hat, der sollte den Wert freier Meinungsäußerung besonders schätzen und einen stärkeren Willen zur Partizipation entwickeln.

Hypothese BF5: Je umfangreicher die Auseinandersetzungen mit Autoritäten im Schulkontext sind, desto postmaterialistischer sind die Wertorientierungen im 30. Lebensjahr.

\section{Zum Einfluss manifester Sozialisationserfahrungen in der J ugend}

Die manifeste politische Sozialisation in der Schule vermittelt politisches Wissen und Kompetenzen, die eine Entwicklung postmaterialistischer Wertansprüche positiv beeinflussen sollte (s.o.).

Hypothese BF6: Je mehr die Schülerinnen und Schüler im Unterricht mit gesellschaftlichen Themen in Kontakt kommen, desto postmaterialistischer sind die Wertorientierungen im 30. Lebensjahr.

Eine positive Bewertung früher politischer Partizipation, ein hohes Prestige politischer Beteiligung im sozialen Umfeld sowie eigene Partizipationserfahrungen sollten ebenfalls zu stärker postmaterialistischen Präferenzen führen:

Hypothese BF7: Je mehr politische Partizipationserfahrungen in der Jugendzeit gesammelt werden, desto postmaterialistischer sind die Wertorientierungen im 30. Lebensjahr. 
Hypothese BF8: Je positiver die Einstellung zur politischen Partizipation, desto postmaterialistischer sind die Wertorientierungen im 30. Lebensjahr.

Hypothese BF9: Je positiver das perzipierte Image politischer Partizipation in der Schulklasse, desto postmaterialistischer sind die Wertorientierungen im 30. Lebensjahr.

\section{Zum Einfluss sekundärer Sozialisationserfahrungen}

Der Lebenszyklus-Hypothese zufolge werden politische Orientierungen von der ökonomischen und sozialen Lebenslage beeinflusst. Veränderte Bedingungen und Rollenerwartungen im beruflichen und privaten Leben nach der mittleren Adoleszenz sollten daher Einfluss auf die individuellen Wertorientierungen haben.

Hypothese BS1: Je höher der erreichte Bildungsabschluss ist, desto postmaterialistischer sind die Wertorientierungen im 30. Lebensjahr ${ }^{33}$.

Relevant sollte darüber hinaus die aktuelle sozio-ökonomische Situation sein:

Hypothese BS2: Je besser die gegenwärtige sozio-ökonomische Stellung ist, desto postmaterialistischer sind die Wertorientierungen.

Umgekehrt lässt sich vermuten, dass mit der sozialen Verantwortung, die mit der Betreuung von Kindern verbunden ist, postmaterialistische Werte an Bedeutung verlieren und stattdessen materialistische Wertorientierungen eine stärkere Zustimmung erfahren:

Hypothese BS3: Je größer die Beanspruchung durch die Betreuung von Kindern, desto materialistischer sind Wertorientierungen im 30. Lebensjahr.

33 Inglehart selbst sieht den eigenen Bildungsabschluss zwar als einen Indikator für die ,formative' Phase an, wie weiter oben dargelegt, (vgl. Hypothese AS1) wird hier die Variable Bildung aber als zeitlich nach der ,formativen' Phase angesiedelt betrachtet. 
Neben der privaten Verantwortung dürften auch Erfahrungen mit beruflichen Bindungen und Sachzwängen im späteren Lebensverlauf zu vermehrt materialistischen Orientierungen führen (vgl. Bürklin et al. 1994):

Hypothese BS4: Je mehr berufliche Erfahrungen gesammelt wurden, desto materialistischer sind die Wertorientierungen.

Schließlich sollten auch Partizipationserfahrungen nach der Adoleszenz Auswirkungen auf die Ausprägung der Wertorientierung haben:

Hypothese BS5: Je mehr politische Partizipationserfahrungen nach der Jugendzeit gesammelt werden, desto postmaterialistischer sind die Wertorientierungen im 30. Lebensjahr.

Zusätzlich ist auch im Hinblick auf Wertorientierungen von einer geschlechtspezifischen Sozialisation auszugehen:

Hypothese BG1: Die Erklärungsstruktur- und Kraft der Erklärungsfaktoren für Wertorientierungen weist geschlechtsspezifische Unterschiede auf.

\section{Interaktionseffekte zur Stabilität der Wertorientierungen}

Auch bei der Stabilität der Wertorientierungen können Interaktionseffekte erwartet werden (vgl. Al1 bis Al2):

Hypothese BI1: Bei starkem Politikinteresse ist die Stabilität der Wertorientierungen höher, als bei schwachem Politikinteresse.

Hypothese BI2: die Stabilität der Wertorientierungen steigt mit zunehmender Bildung an. 


\section{Daten und Methode}

\section{1 Überblick über die Analysestrategie}

Die Überprüfung der in Kapitel 5 formulierten Hypothesen erfolgt in 5 Schritten, unterteilt in deskriptive und analytische Abschnitte (s. Kapitel 7). In einem ersten Schritt werden die wichtigsten deskriptiven Kennwerte in Form von Verteilungen, Mittelwerten und Veränderungsraten der Zielvariablen zwischen den beiden Messzeitpunkten 1985 und 1997 dargestellt.

Im zweiten Schritt wird die Stabilität des politischen Interesses und der Wertorientierungen im Erwachsenenalter vom 30. bis zum 43. Lebensalter untersucht. In der vorliegenden Arbeit wird die Stabilität der Variablen zwischen $t$ und $t+1$ über Korrelationskoeffizienten - in diesem Fall polychorische Korrelationen - und die Regressionskoeffizienten berechnet. Die formale Definition von Stabilität wird im folgenden Kapitel dargelegt.

Der dritte Schritt dient der Untersuchung der bivariaten Zusammenhänge zwischen den abhängigen und unabhängigen Variablen.

Im anschließenden vierten Schritt werden Pfadmodelle berechnet, mit denen überprüft wird, welchen Einfluss latente und manifeste Sozialisationserfahrungen in der mittleren Adoleszenz im Vergleich zu späteren Erfahrungen auf politisches Interesse und politische Wertorientierungen im Erwachsenenalter haben. Mit diesem Verfahren kann nicht nur eine Zielvariable untersucht werden, sondern es werden simultan mehrere abhängige Variable analysiert. Somit ist es möglich, neben direkten Einflüssen auch indirekte Effekte auf die Zielvariablen ermitteln.

Der letzte fünfte Schritt schließlich untersucht die Frage, ob den Hypothesen entsprechende Gruppenunterschiede bezüglich der Stabilitäten vorliegen. Dazu werden die jeweiligen Stabilitätskoeffizienten durch multiple Gruppenvergleiche simultan einem Modelltest unterzogen. Mit diesem Verfahren kann getestet werden, ob die Stabilitätskoeffizienten über die Gruppen hinweg variieren (vgl. Jöreskog et al. 2001: 9f., Reinecke 2005: 64f.). 
Da die meisten der verfügbaren Variablen kategoriales Datenniveau aufweisen, und eine gleichzeitige Verwendung metrischer und ordinaler Variablen zu Problemen führt, werden die metrischen Variablen (Prestigescores, Dauer der Erwerbstätigkeit, Werte der Intelligenztests) im folgenden entlang der Quartilsgrenzen kategorisiert.

Alle weiteren datenanalytischen Vorgehensweisen werden im Kontext mit den im nächsten Kapitel (Kapitel 7) dargestellten Auswertungen erläutert und begründet.

Für die Untersuchung der im letzten Kapitel entwickelten Fragestellung werden Daten benötigt, die eine Analyse der individuellen Stabilität politischer Orientierungen ermöglichen. Folglich ist die Untersuchung auf Panelstudien angewiesen: Nur wenn die Messung der Wertorientierungen bzw. des politischen Interesses an den gleichen Untersuchungspersonen mehrmals wiederholt wird, lassen sich Veränderungen in den individuellen Orientierungsstrukturen ermitteln. Darüber hinaus sollten die Panelstudien einen möglichst langen Zeitraum umfassen, da bei kurzfristigen Wiederholungs-Befragungen (wie etwa bei den bundesdeutschen Wahlstudien oder auch den ,Political-Action'-Studien) langfristige Entwicklungen nicht untersucht werden können.

Will man die Bedeutung der Adoleszenz bei der Entwicklung individueller politischer Orientierungen untersuchen, dann ist - neben den aktuellen und kurzzeitig zurückliegenden Einflüssen und Erfahrungen - auch die primäre Sozialisation während der Jugendzeit zu berücksichtigen (vgl. Niemi/Junn 1998, Stolle/Hooghe 2004). Nur wenn relevante Erfahrungen aus der primären Sozialisationsphase ermittelt und in Bezug zur individuellen politischen Identität im Erwachsenenalter gesetzt werden, sind Aussagen über die langfristigen Auswirkungen der primären Sozialisationsprozesse zulässig.

Wie bereits dargelegt, scheitert eine direkte Überprüfung der Sozialisationsthese in aller Regel an der mangelhaften Datenlage (vgl. Kapitel 4). Prospektive, mehrere Dekaden umfassende Panel-Studien, die sowohl politische Orientierungen erfassen als auch Informationen über frühe 
Sozialisationserfahrungen im Elternhaus, in der Schule oder Peergroup enthalten, sind nur selten verfügbar.

Um die Wirkung früher Sozialisationseinflüsse zumindest indirekt zu erfassen, wird in der sozialwissenschaftlichen Praxis daher oft auf das Verfahren der Kohortenanalyse zurückgegriffen (z.B. Mnich 1989, Inglehart 1989, Bürklin et al. 1994, zum Verfahren der Kohortenanalyse: Glenn 1977, Plum 1982).

Ausgehend von der Annahme, dass Angehörige verwandter Geburtsjahrgänge unter ähnlichen Bedingungen und in ähnlicher Weise sozialisiert werden ${ }^{34}$, wird in der Kohortenanalyse die Zugehörigkeit zu bestimmten Geburtsjahrgängen als Proxy für Sozialisationserfahrungen benutzt. Wenn dauerhafte Stabilität der formativen Prägungen vorausgesetzt werden kann, dann sollten differierende soziale, politische oder ökonomische Sozialisationsbedingungen in der Jugend zu Unterschieden zwischen den jeweiligen Kohorten führen (=Kohorteneffekt) ${ }^{35}$. Gesellschaftlicher oder politischer Wandel vollzieht sich nach dem Kohorten- oder Generationsansatz aus diesem Grunde vornehmlich über die veränderten Einstellungen und Orientierungen der jeweils nachwachsenden Generationen (vgl. Mannheim 1928).

In vielen Kohortenanalysen der neueren Zeit wurden Generationenunterschiede entdeckt, die als Beleg für die dauerhafte Wirksamkeit früher Sozialisation gedeutet werden (vgl. etwa für Deutschland: Klein/Arzheimer 1997, Becker 2002, Arzheimer 2006, Klein 2004). Welche konkreten inhaltlichen Sozialisationserfahrungen hinter den beobachteten Kohorteneffekten stehen, bleibt diesen Analysen zufolge jedoch meist unklar (Arzheimer 2006: 325). Die Frage beispielsweise, ob tatsächlich die wirtschaftlichen Verhältnisse in der ,formativen Phase‘ für die beobachteten

34 Diese Annahme ist allerdings problematisch, da zum Teil erhebliche Sozialisationsunterschiede - beispielsweise im Hinblick auf die Geschlechtszugehörigkeit bestehen. Das heißt, dass unter Umständen die Sozialisationsdifferenzen innerhalb einer Generation größer sind, als zwischen den Generationen.

35 Die Kohortenanalyse ist allerdings mit einer Reihe methodischer Probleme, wie etwa der Trennung von sich überlagernden Kohorten,- Lebenszyklus- und Periodeneffekten behaftet, vgl. dazu Mayer/Huinink 1990. 
Generationendifferenzen bei Wertorientierungen ausschlaggebend sind, wie Inglehart behauptet, oder aber, ob andere Ursachen - wie beispielsweise veränderte Erziehungspraktiken in bestimmten Kohorten - für Werteveränderungen verantwortlich sind, wird in diesen Kohortenanalysen nicht beantwortet.

Für die vorliegende Analyse wird daher eine Panelstudie verwendet, die sowohl langfristig angelegt ist, als auch gleichzeitig Variablen zur Messung relevanter primärer Sozialisationserfahrungen enthält.

\subsection{Die Stichprobe}

Grundlage der folgenden Datenanalyse bildet eine Stichprobe ehemaliger nordrhein-westfälischer Gymnasiasten, die an einem dreiwelligen Langzeitpanel zum schulischen, beruflichen und privaten Werdegang zwischen 1969/70 und 1997 teilnahmen.

Die Erstbefragung der Gymnasiasten (ZA-Nr. 600) sowie eine parallel durchgeführte Elternbefragung (ZA-Nr. 639) fand zwischen Dezember 1969 und März 1970 statt. Zu diesem Zeitpunkt waren die befragten Schüler zwischen 15 und 16 Jahre alt und besuchten die 10. Schulklasse.

Finanziert wurde die Primärstudie vom Land NRW und durchgeführt unter der Leitung von Hans-Joachim Hummell vom Forschungsinstitut für Soziologie der Universität Köln. Die Stichprobenziehung erfolgte in Form einer zweistufigen Klumpenauswahl von Schülerinnen und Schülern der 10. Jahrgangsstufe aus insgesamt 121 verschiedenen Klassen an 68 nordrhein-westfälischen Gymnasien. Die Ausgangsstichprobe umfasste insgesamt 3.240 Schülerinnen und Schülern, die mit einem standardisierten Fragebogen schriftlich im Klassenverband interviewt wurden ${ }^{36}$.

\footnotetext{
36 Mein herzlicher Dank gilt Heiner Meulemann für die freundliche und großzügige Bereitstellung des Datensatzes sowie Oliver Watteler für die Erstellung der umfangreichen Datendokumentation und die große Hilfsbereitschaft bei der Beantwortung der Fragen zum Datensatz. Eine ausführliche Projektbeschreibung ist in der DFG-Projektbeschreibung zu finden, vgl. Birkelbach et al. 2000.
} 
Die Fragebögen dieser Erhebung enthalten in erster Linie Fragen zu schulischen Leistungen und Vorlieben sowie zu den Zukunftsplänen der Schüler. Daneben wurden auch Fragen zur sozialen Herkunft sowie zu familialen und schulischen Aspekten gestellt, die Auskunft über die Sozialisationserfahrungen der Gymnasiasten geben.

Zusätzlich wurde im Kontext der Primärbefragung ein Intelligenz-Strukturtest (Amthauer 1953) mit den Schülern durchgeführt.

Die zeitgleich durchgeführte Elternbefragung erfolgte schriftlich und enthielt im wesentlichen Fragen zur schulischen Leistung der Kinder, ihrer Erziehung und zu den weiteren schulischen Aspirationen der Kinder ${ }^{37}$.

Die erste Wiederbefragung zum Thema „Lebenserfolg und Erfolgsdeutung im frühen Erwachsenenalter" (ZA-Nr. 1441) fand in den Jahren 1984/85 und unter Finanzierung der DFG statt. Die Befragung wurde am Zentralarchiv für empirische Sozialforschung unter der Federführung von Heiner Meulemann konzipiert und umfasst eine Stichprobe von 1987 ehemaligen Gymnasiasten, die jetzt etwa 30 Jahre alt waren. Die zweite Panelwelle erreichte damit eine Stichprobenausschöpfung von 61,4\% (zur Panelmortalität s.u.). Mit den standardisierten, persönlichen Befragungen wurde das Getas-Institut aus Bremen beauftragt, das die Interviews im Zeitraum zwischen September 1984 und Juni 1985 durchführte. Die zweite - ebenfalls von Heiner Meulemann geleitete und von der DFG finanzierte - Wiederbefragung erfolgte 1996/97. Im Unterschied zu den vorangegangenen Befragungen fand diese Panelerhebung als telefonische Befragung statt. An dieser letzten Erhebung nahmen insgesamt 1596 Befragte teil, was einer Ausschöpfung von 49,3\% der Ausgangsstichprobe entspricht. Durchgeführt wurde sie unter Leitung der Primärforscher in der Zeit von Dezember 1996 bis Juni 1997 im CATI ${ }^{38}$-Labor des Instituts für Angewandte Sozialforschung der Universität Köln. Die inhaltlichen Schwerpunkte der beiden Wiederbefragungen lagen auf den beruflichen und privaten Lebensverläufen der ehemaligen Gymnasiasten. In

\footnotetext{
$37 \mathrm{Ob}$ von den Eltern die Mutter oder der Vater befragt wurde, ist leider nicht dokumentiert. Zusätzlich zu den Schüler- und Elternbefragungen fand eine Befragung der Klassenlehrer (ZANr. 640) und Direktoren (ZA-Nr. 969) statt.

${ }^{38} \mathrm{CATI}=$ Computer Assisted Telephone Interviewing.
} 
beiden Panelwellen wurden aber auch einige politische Orientierungen erhoben.

\subsection{Operationalisierung}

Für die folgenden Analysen werden unter anderem Pfadmodelle berechnet (vgl. Kapitel 7.2). Um die Möglichkeit der Messfehlerkorrektur innerhalb dieses Verfahrens zu nutzen, werden, sofern möglich, multiple Indikatoren zur Messung der jeweiligen Konstrukte verwendet. Das in Sekundäranalysen übliche Problem des Findens geeigneter Indikatoren, besteht auch in der hier vorliegenden Analyse. So war es leider nur in einigen Fällen möglich, in den verfügbaren Datensätzen geeignete multiple Indikatoren zur Bestimmung der jeweiligen Konstrukte ausfindig zu machen. Für die verbleibenden Konstrukte wird daher auf daher auf Single-Indikator-Messungen zurückgegriffen und damit für die betreffenden Variablen auf Berücksichtigung der Messfehler verzichtet. Eine Übersicht über die verwendeten Konstrukte und Indikatoren befindet sich am Ende des Kapitels (Tabelle 6.5).

\subsubsection{Die Messung der abhängigen Variablen}

Zur Messung der abhängigen Variablen wird die zweite und dritte Panelwelle (ZA-Nr. 1441 und 4228) der vorliegenden Stichprobe verwendet. Die genauen Frageformulierungen und Kodierungen sind unten aufgeführt (Fragebogentext und Kodierung 6.1 und 6.2).

\subsubsection{Politisches I nteresse}

Ein in Bevölkerungsumfragen sehr häufig verwendetes Instrument zur Messung des Politikinteresses ist die Frage nach dem subjektiven politischen Interesse (siehe Fragebogentext und Kodierung 6.1, Punkt a). 
Fragebogentext und Kodierung 6.1:

Variable "Politikinteresse" und ,Wichtigkeit von Politik und öffentlichem Leben“

\section{a) Subjektives politisches I nteresse:}

- Wie stark interessieren Sie sich für Politik?

Antwortvorgaben und Kodierung:

(1) Überhaupt nicht

(2) Wenig

(3) Mittel

(4) Stark

(5) Sehr stark

\section{b) Wichtigkeit von Politik und öffentlichem Leben:}

Nun hätte ich gerne von Ihnen gewusst, wie wichtig für Sie die folgenden Lebensbereiche sind, die ich Ihnen nun vorlese.. Sagen Sie mir bitte jetzt auf einer Skala von 1 bis $7 \mathrm{zu}$ jedem Lebensbereich für wichtig Sie inn halten: 1 bedeutet dabei "unwichtig“ und 7 bedeutet "sehr wichtig“. Und die Zahlen dazwischen stufen die Wichtigkeit $a b$.

\section{- ...Politik und öffentliches Leben?}

(Die Reihenfolge in der die verschiedenen Lebensbereicht abgefragt werden ist folgende: eigene Familie und Kinder; Beruf und Arbeit; Freizeit und Erholung; Freunde und Bekannte; Verwandschaft; Religion und Kirche; Politik und öffentliches Leben; Nachbarschaft)

Antwortvorgaben und Kodierung:

(1) Überhaupt nicht bis (7) Sehr wichtig

Da die Messung des Politikinteresses durch einen Single-Indikator jedoch mit Messfehlern behaftet sein dürfte, ist hier mit Reliabilitätsproblemen ${ }^{39} \mathrm{zu}$

39 Darüber hinaus liegen bei Fragen zum politischen Interesse Validitätsprobleme vor, die unter anderem durch die Unschärfe des Politikbegriffs entstehen. So stellt sich zum Beispiel die Frage, was die Befragten inhaltlich meinen, wenn sie angeben, sie seien an Politik interessiert - oder auch nicht interessiert (vgl. van Deth 1990: 283f.). Verstehen die Befragten unter Politikinteresse regelmäßiges Zeitungslesen, politische Diskussionen mit Freunden oder Kollegen oder auch nur die regelmäßige Beteiligung an politischen Wahlen? Darüber hinaus besteht die Gefahr, dass Effekte der sozialen Erwünschtheit die Qualität des Messinstrumentes einschränken (vgl. Kapitel 4). 
rechnen. Daher sollte, falls möglich, auf die Verwendung von multiplen Indikatoren zurückgegriffen werden.

Ein zweiter Indikator, der im vorliegenden Datensatz verfügbar ist und zur Messung des politischen Interesses herangezogen werden könnte, ist die Variable ,Wichtigkeit von Politik und öffentlichem Leben' (siehe Fragebogentext und Kodierung 6.1, Punkt b). Der Indikator misst die Bedeutung von Politik und öffentlichem Leben, indem er die Wichtigkeit von Politik im Vergleich zu anderen Lebensbereichen abfragt (s.u. Fragebogentext und Kodierung 6.1).

Allerdings ist diese Variable nicht problemlos als zweiter Indikator zur Messung des politischen Interesses verwendbar, da konzeptionelle Unterschiede zum subjektiven politischen Interesse vermutet werden können. In einer Untersuchung zum Zusammenhang zwischen den Variablen des subjektiven Politikinteresses und der Bedeutung von Politik kommt van Deth (2000) zu dem Ergebnis, das diese beiden Aspekte des Interesses an Politik zwar positiv korrelieren, analytisch aber zu trennen seien. So stellt er fest, dass Einflüsse des sozialen Status der Befragten (Bildung, z. T. Einkommen) sowie die Geschlechtszugehörigkeit für das subjektive politische Interesse sehr viel relevanter sind, als für die individuelle Bedeutung der Politik (vgl. van Deth 2000: 123). Bei der Höhe der erklärten Varianz erreichte zudem das subjektive politische Interesse in seiner Analyse erheblich bessere Werte als die Variable zur Bedeutung der Politik.

Auf die Frage, ob sich die beiden beschriebenen Variablen für die vorliegende Arbeit nun als multiple Indikatoren zur Messung des theoretischen Konstruktes ,Politikinteresse' eignen, d.h. ob sie sich über beide Messzeitpunkte hinweg zu Konstrukten bündeln lassen, wird weiter unten eingegangen (s. Kapitel 7.1). 


\subsubsection{Wertorientierungen}

Postmaterialistische Wertorientierungen wurden in der Stichprobe der früheren Gymnasiasten mit dem Standardinstrument des Inglehart-Indexes erhoben (siehe Fragebogentext und Kodierung 6.2):

\section{Fragebogentext und Kodierung 6.2: Variable ,Postmaterialismus"}

„Nun zu etwas anderem: Auch in der Politik kann man nicht alles auf einmal haben. Ich möchte Ihnen nun vier Ziele vorlesen, die man in der Politik verfolgen kann. Welches der nun folgenden Ziele ist I hnen persönlich am wichtigsten?

Und welches Ziel erscheit Ihnen am zweitwichtigsten?

Und welches Ziel erscheint Ihnen am drittwichtigsten?“

1. Aufrechterhaltung von Ruhe und Ordnung in diesem Land (materialistisch)

2. Mehr Einfluss der Bürger auf die Entscheidung der Regierung (postmaterialistisch)

3. Kampf gegen die steigenden Preise (materialistisch)

4. Schutz des Rechtes auf freie Meinungsäußerung (postmaterialistisch)

\section{Kodierung:}

(1) Materialisten

(2) M-Mischtyp

(3) PM- Mischtyp

(4) Postmaterialisten

Auf Basis der von den Befragten gebildeten Rangreihe gesellschaftspolitischer Ziele wurden die vier von Inglehart unterschiedenen Wertetypen codiert: Als Postmaterialisten wurden diejenigen eingruppiert, welche die postmaterialistischen Items als wichtigstes und zweitwichtigstes Ziel nannten. Bewerteten die Befragten dagegen die beiden materialistischen Items als am wichtigsten und zweitwichtigsten, so wurden sie zur Gruppe der Materialisten zusammengefasst. In die Kategorie der materialistischen Mischtypen wurden diejenigen eingeordnet, die ein materialistisches Item auf die erste und ein postmaterialistisches Item auf die zweite Rangposition setzten. Für die postmaterialistischen Mischtypen schließlich steht ein postmaterialistisches Ziel an erster und ein materialistisches Ziel an zweiter Stelle der Rangreihe. Die Kodierung der Wertetypen erfolgt den Inglehartschen Annahmen zufolge aufsteigend nach dem Grad des Postmaterialismus. 


\subsubsection{Die Messung der unabhängigen Variablen}

Für die Messung der erklärenden Variablen werden insgesamt drei Datensätze herangezogen. Die Variablen zu den jugendlichen Sozialisationserfahrungen werden den Daten der Primärbefragung der Schüler und der Elternbefragung entnommen (ZA-Nr. 600 und 639). Zur Messung der Sekundäreinflüsse wird die zweite Panelwelle (ZA-Nr. 1441) der Stichprobe verwendet. Die genauen Frageformulierungen und Kodierungen werden auch hier im folgendem zitiert (Fragebogentext und Kodierung 6.3 bis $6.9)$.

\subsubsection{Sozio-ökonomischer Status des Elternhauses}

Als wesentliche Dimensionen des sozio-ökonomischen Status (SES) werden in der Sozialstrukturanalyse die Variablen Bildung, Stellung im Beruf und Einkommen betrachtet. Diese Merkmale beeinflussen die ökonomischen, sozialen und kulturellen Lebenschancen des einzelnen in der Gesellschaft erheblich (vgl. Geißler 2002).

Der Status des Elternhauses während der Jugendzeit der Befragten wird in der folgenden Untersuchung anhand des Berufsprestiges des Vaters in Form der Prestige-Skala nach Treiman ${ }^{40}$ (1977) und der Schulbildung beider Elternteile erhoben. Alle drei Variablen wurden der Elternbefragung entnommen, da die Angaben Eltern hier zuverlässiger sein dürften als die der Jugendlichen. Die Messung des väterlichen Berufsprestiges erlaubt neben der Schulbildung die Bestimmung der hierarchischen Position des Elternhauses und damit die ,formative Sicherheit' unter der die Jugendlichen aufwachsen (siehe Fragebogentext und Kodierung 6.3) ${ }^{41}$.

\footnotetext{
40 Dabei handelt es sich um eine international vergleichende Prestige-Skala, die berufliche Tätigkeiten entsprechend ihrem Prestige und ihres sozialen Ansehens eingestuft (vgl. Treiman 1977).

${ }^{41}$ Zwar wurde auch die berufliche Stellung der Mutter erhoben, da aber in der Stichprobe nur vergleichsweise wenige berufstätig Mütter vorhanden sind und die Gesellschaft der 60er J ahre des letzten Jahrhunderts zudem stark patriarchalisch organisiert war, wird auf die Verwendung des Berufsprestige der Mütter hier verzichtet. Auch auf die Verwendung des Einkommens der
} 
Fragebogentext und Kodierung 6.3: Die Variablen zu den sozioökonomischen Ressourcen während der Jugendzeit (aus Elternbefragung, ZA. Nr. 0639)

a) Prestige - Scores nach Treiman (1977):

Kodierung $^{42}$ :

(0) 0 bis 31

(1) 32 bis 41

(2) 42 bis 55

(3) 56 bis 78

b) und c) Schulbildung Vater und Mutter:

- Welche Schulen haben Sie und Ihr Ehepartner zuletzt besucht?

Kodierung: Die ursprünglich 13 Kategorien der elterlichen Schulabschlüsse wurden in drei Kategorien zusammengefasst

(1) Volksschule (mit und ohne Lehre)

(2) Mittelschule bis höhere Schule ohne Abitur,

(3) Höhere Schule mit Abitur bis Universität mit Abschluss.

\subsubsection{Kognitive Kompetenz in der J ugend}

Um die kognitive Kompetenz der Jugendlichen zu erfassen, werden die Ergebnisse der Intelligenz-Strukturtests (Amthauer 1953) verwendet, die im Anschluss an die Primärerhebung der ehemaligen Schüler durchgeführt wurden. Von den vier Subtests ${ }^{43}$ wurden die beiden Subtests zur sprachlichen Intelligenz ausgewählt, da man davon ausgehen kann, dass vor allem die verbalen Fähigkeiten für die Auseinandersetzung mit politischen Gegenständen und Zielen von Relevanz sind. Es handelt sich hierbei um den

Eltern wird verzichtet, um die Fallzahlen der Untersuchungsstichprobe nicht unnötig weiter zu reduzieren.

42 Um Berechnungen der polychorischen Korrelationen zu ermöglichen, wurden die metrischen Variablen (Prestigescores, Dauer der Erwerbstätigkeit, Werte der Intelligenztests) entlang der Quartilsgrenzen kategorisiert.

${ }^{43}$ Neben den sprachlichen Intelligenztests wurden zwei Subtests zur mathematischen Intelligenz durchgeführt. 
sogenannten ,Wortauswahl-' und den ,Analogie-Test'. Mit dem Wortauswahltest wird das Begreifen von sprachlichen Bedeutungsinhalten sowie das ,Gefühl' für Sprache gemessen. Die verbale Kombinationsfähigkeit, Beweglichkeit, Klarheit und Folgerichtigkeit im Denken wird mithilfe des Analogietests ermittelt (Beispiele siehe in Fragebogentext und Kodierung $6.4)$.

\section{Fragebogentext und Kodierung 6.4:} Kognitive Kompetenz in der Jugend

\section{a) Kognitive Kompetenz in J ugendzeit:}

\section{Wortauswahl-Test:}

- Instruktion: von 5 vorgegebenen Wörtern sind 4 in gewisser Weise ähnlich. Sie sollen das fünfte Wort finden, das den anderen in dieser Weise nicht ähnlich ist

Beispiel: a) Tisch b) Stuhl c) Vogel d) Schrank e) Bett

Kodierung: Erreichte Punktzahl im Test
(0) 0 bis 9
(1) 10 bis 11
(2) 12
(3) 13 bis 19

Analogie-Test:

- Instruktion: Es werden Ihnen 3 Wörter vorgegeben. Zwischen dem ersten und zweiten Wort besteht eine gewisse Beziehung. Zwischen dem dritten und einem der fünf Wahlwörter besteht eine ähnliche Beziehung. Dieses Wort sollen Sie finden.

Beispiel: Wald : Bäume = Wiese : ..?.. a) Gräser b) Heu c) Futter d) Grün e) Weide

Kodierung: Erreichte Punktzahl im Test

(0) 0 bis 9

(1) 10 bis 11

(2) 12 bis 14

(3) 15 bis 20 


\subsubsection{Latente politische Sozialisation im Elternhauses}

Zur Messung der latenten politischen Sozialisation in der Familie werden zwei Konstrukte verwendet: Das Familienklima und die Erziehungsziele aus Sicht der Eltern.

Die Qualität des Familienklimas wird mit den Variablen ,Kindererziehung wie bei den Eltern?' und ,Spannungen zu Hause?' erhoben. Diese Variablen geben Auskunft darüber, wie positiv oder negativ Atmosphäre in der Familie von den Jugendlichen empfunden wird (siehe die Punkte $a$ und $b$ in Fragebogentext und Kodierung 6.5).

Das zweite Konstrukt wird aus Variablen gebildet, die der Elternbefragung entnommen wurden. Es handelt sich dabei um Fragen zu Erziehungszielen, die Eltern der höheren Schule zuweisen. Konkret geht es zum einen um die Frage, ob es Aufgabe der Schule sei, zur Kritik an der bestehenden Gesellschaft zu erziehen. Zum anderen wird danach gefragt, ob die höhere Schule die Fähigkeit zur selbständigen Problemlösung vermitteln müsse. Die Antworten der Eltern auf diese Fragen werden als Surrogat für die eigenen Erziehungspraktiken- und ziele bei der Kindererziehung verwendet (siehe die Punkte $\mathrm{c}$ und $\mathrm{d}$ in Fragebogentext und Kodierung 6.5). 
Fragebogentext und Kodierung 6.5:

Latente politische Sozialisation in der Familie

\section{Familienklima:}

a) Kindererziehung wie bei den Eltern

- Würden Sie Ihre Kinder genauso erziehen, wie I hre Eltern Sie erzogen haben?

Kodierung:

(1) Ganz anders

(2) Anders

(3) Ungefähr so

(4) Genau so

b) Spannungen zu Hause?

- Zwischen Eltern und Kindern gibt es in den meisten Familien Spannungen: Wie ist das bei Ihnen zu Hause?

\section{Kodierung:}

(1) Häufiger unerträgliche Spannungen

(2) Häufiger ziemlich schwerwiegende Spannungen

(3) Nicht so große Spannungen

(4) Eigentlich keine Spannungen

c) und d) Erziehungsziel zu selbständigen Denken und Handeln

- In der Diskussion über die Reform der höheren Schule werden sehr verschiedene Gesichtspunkte in den Vordergrund gerückt. Wir haben eine Reihe von möglichen Zielsetzungen zusammengestellt. Geben Sie bitte für jeden dieser Punkte an, für wie wichtig Sie es halten, dass sich die höhere Schule diese Aufgabe annimmt. 5 soll hierbei jeweils heißen "besonders wichtig“, 0 „weniger wichtig“, 4, 3, 2, 1 liegen entsprechend dazwischen.

....Aufgabe der höheren Schule: Zur Kritik an der bestehenden Gesellschaft zu erziehen

....Aufgabe der höheren Schule: die Fähigkeit vermitteln, Probleme selbständig zu lösen

Kodierung bei beiden Items:

(0) Weniger Wichtig bis (5) Besonders wichtig 


\subsubsection{Latente politische Sozialisation in der Schule}

Um das Ausmaß der Auseinandersetzungen mit Autoritäten im Schulalltag zu bestimmen, wird eine Variable verwendet, die zählt, mit wie vielen Protestmaßnahmen Schüler auf Bestrafungen in der Klasse im letzten Jahr reagiert haben (siehe Fragebogentext und Kodierung 6.6). Es wird davon ausgegangen, dass diese mit dem Ausmaß der Auseinandersetzung mit Autoritäten positiv korreliert.

\section{Fragebogentext und Kodierung 6.6:}

Latente politische Sozialisation in der Schule

\section{a) Protesterfahrungen in der Klasse ${ }^{44}$}

- Hat Ihre Klasse im letzten Jahr schon einmal etwas derartiges unternommen?

- Mit dem Lehrer/der Lehrerin diskutieren, um den / die es sich handelt?

- Andere Lehrer einschalten?

- Sich an den Direktor wenden?

- Kollektives Stören des Unterrichts?

- Beitrag in der Schülerzeitung?

- Antrag im Schülerparlament?

- Sich an die Öffentlichkeit wenden (Flugblätter, Lokalzeitung)?

- Eltern bitten, etwas gemeinsames zu unternehmen?

Kodierung: Die Angaben wurden in drei Kategorien zusammengefasst.

(0) Keine Protesterfahrungen

(1) Eine Protesterfahrung

(2) Zwei und mehr Protesterfahrungen

44 Hinsichtlich der genannten Protesterfahrungen lässt sich einwenden, dass nur die ersten drei Aktivitäten latente Sozialisationserfahrungen darstellen, während es sich bei den letzten drei Protesthandlungen um eher manifeste politische Erfahrungen handelt. Da die letzten drei Aktivitäten jedoch nur von einer kleinen Minderheit genannt wurden (Nennungen im einstelligen Prozentbereich), wird diese Variable weiterhin als latentes Sozialisationsmerkmal betrachtet. 


\subsubsection{Manifeste politische Sozialisation in der Schule}

Die manifeste politische Sozialisation in der Schule wird mit insgesamt vier Variablen gemessen. Drei dieser Variablen sollen das Ausmaß erfassen, mit dem die Gymnasiasten im Unterricht mit sozialwissenschaftlichen Themen in Kontakt geraten.

Zum einen wird ein Index konstruiert, der die Einstellung zu sozialwissenschaftlichen Fächern im Unterricht ermittelt. Dazu wurden Fragen, verwendet, in denen die Schüler gefragt wurden, welche Fächer sie am liebsten haben und welche sie am wenigsten mögen (die genaue Variablenkonstruktion ist in Fragebogentext und Kodierung 6.7, Punkt a wiedergegeben).

Die zweite Variable besteht aus der aktuellen Durchschnittsnote der sozialwissenschaftlichen Fächer (siehe Fragebogentext und Kodierung 6.7, Punkt b).

Das dritte Merkmal, das Informationen zur manifesten politischen Sozialisation in der Schule liefern soll, ist eine Variable aus der Elternbefragung. Hier wurden die Eltern gefragt, inwiefern Sie ihre Kinder für begabt in sozialwissenschaftlichen Fächern halten (siehe Fragebogentext und Kodierung 6.7, Punkt c).

Die vierte Variable schließlich misst das von den Schülern wahrgenommene Image, das politisches Engagement in der Schulklasse genießt (siehe Fragebogentext und Kodierung 6.7, Punkt d). 


\section{Fragebogentext und Kodierung 6.7:}

Manifeste politische Sozialisation in der Schule

\section{a) Beliebteste vs. unbeliebteste Fächer aus dem sozialwissenschaftlichen Bereich}

- Kreuzen Sie bitte die drei Fächer an, die Sie am liebsten haben.

- Kreuzen Sie bitte die drei Fächer an, die Sie am wenigsten gern haben

Kodierung: Index aus den Nennungen der Fächer Geschichte und Sozialkunde als beliebteste bzw. unbeliebteste Fächer

$(+2)$ Geschichte und Sozialkunde als Lieblingsfach

$(+1)$ Geschichte oder Sozialkunde als Lieblingsfach

( 0) Weder Geschichte noch Sozialkunde als beliebteste oder unbeliebteste Fächer genannt

( - 1) Geschichte oder Sozialkunde als unbeliebtes Fach

( -2) Geschichte und Sozialkunde als unbeliebtes Fach

b) Durchschnittsnote in sozialwissenschaftlichen Fächern, bestehend aus Geschichte, Sozialkunde

Durchschnittsnote sozialwissenschaftliche Fächer

Kodierung: Wertebereich von (1) 'mangelhaft' bis (5) 'sehr gut'

c) Begabung in sozialwissenschaftlichen Fächern (Elternmeinung)

- Es ist häufig so, dass Kinder in bestimmter Richtung mehr begabt sind, während ihnen andere Gebiete weniger liegen. Wie beurteilen Sie die Begabung Ihres Sohnes/lhrer Tochter in den folgenden Bereichen... Sohn/Tochter ist in Literatur oder Geschichte...

\section{Kodierung:}

(1) Nicht so begabt bis unbegabt

(2) Durchschnittlich begabt

(3) Besonders begabt

d) Wahrgenommene Bewertung von politischem Engagement in der Schulklasse

- Welche der folgenden Dinge werden hier in der Klasse besonders geschätzt? ... Politisches Engagement?

\section{Kodierung:}

(1) Unwichtig

(2) Wichtig

(3) Weniger wichtig 
(4) Sehr wichtig

\subsubsection{Weitere manifeste Sozialisationserfahrungen in J ugend: Politische Partizipation in der Jugend}

Die Einstellung der Jugendlichen zur politischen Teilnahme und die eigene politische Partizipation wird mit insgesamt drei Indikatoren erfasst. Die Messung der Einstellung zur politischen Partizipation geschieht über eine Frage zum Alter, ab dem die Schüler eine eigene, aktive politische Betätigung für möglich halten. Wird ein niedriges Alter genannt, so steht dies für eine positive Einstellung zur politischen Teilnahme (siehe Fragebogentext und Kodierung 6.8, Punkt a).

Das eigene politische Verhalten wird zum einen über die Variablen ,Mitglied in politischer Schülergruppe‘ und ,Mitglied in politischer Gruppe‘ gemessen. Aus diesen beiden Variablen wurde eine neue dichotome Variable gebildet, die den Wert 1 annimmt, wenn mindestens eine der beiden Fragen mit ja beantwortet wurden (siehe Fragebogentext und Kodierung 6.8, Punkt b) ${ }^{45}$. Eine weitere Frage zur politischen Partizipation, betrifft die Teilnahme an Demonstrationen. Hier wurden die Gymnasiasten gefragt, ob sie an einer politischen Demonstration teilgenommen haben (siehe Fragebogentext und Kodierung 6.8, Punkt c).

\footnotetext{
45 Diese Variable enthält über die politische Schülergruppe auch einen Aspekt der manifesten schulischen Sozialisation. Da aber über die Zusammenfassung mit sonstigen politischen Gruppierungen die Zuordnung nicht eindeutig ist, wird die Variable an dieser Stelle unter „weitere politische Sozialisationserfahrungen“ aufgeführt.
} 
Fragebogentext und Kodierung 6.8:

Politische Partizipation in der Jugendzeit

\section{a) Gewünschtes Alter für politische Beteiligung}

- Mit wie viel Jahren sollte man Ihrer Ansicht nach über die folgenden Dinge selbst entscheiden?

...selbst entscheiden über aktive politische Betätigung?

Kodierung: Alter in Jahren

b) Mitgliedschaft in politischer Gruppe

Zusammenfassung der Variablen „Mitglied in politischer Schülergruppe“ und „Mitglied in politischer Gruppe“

- Sind Sie Mitglied einer politischen Schülergruppe?

- Sind Sie Mitglied in einem oder mehreren Vereinen oder in einer Jugendorganisation, wo es eine Mitgliedschaft gibt und wo man Mitgliedsbeitrag bezahlt? Was ist das für ein Verein oder was für eine Organisation.... politische Gruppe?

\section{Kodierung:}

(0) nein (weder noch)

(1) Ja (in politischer Gruppe oder politischer Schülergruppe)

c) Demonstrationsteilnahme 1969/ 1970

- In der letzten Zeit hat es in zahlreichen Städten der BRD öffentliche Demonstrationen gegeben. Haben Sie schon einmal an einer solchen Demonstration teilgenommen?

Kodierung:

(0) Nein

(1) Ja 


\subsubsection{Sekundäre Sozialisationserfahrungen}

Das zwischen dem 15. und 30. Lebensjahr erreichte formale Bildungsniveau wird der ersten Wiederbefragung entnommen. Die Höhe der Bildung zeigt in der Stichprobe der früheren Gymnasiasten naturgemäß eine geringere Variation als im Bevölkerungsdurchschnitt (vgl. Tabelle 6.1, S.87). Dennoch lassen sich Unterschiede im Bildungserfolg finden, wie der vierstufigen Kodierung zu entnehmen ist (Fragebogentext und Kodierung 6.9, Punkt a).

Als Indikator für manifeste politische Sozialisationseinflüsse aus der Zeit nach der mittleren Adoleszenz steht im Datensatz der zweiten Panelwelle die Frage nach einer Demonstrationsteilnahme in den letzten Jahren zur Verfügung (siehe Fragebogentext und Kodierung 6.9, Punkt b).

Die lebenszyklischen Erfahrungen, die im vorliegenden Datensatz erfasst wurden, beziehen sich sowohl auf den privaten als auch auf den beruflichen Bereich.

Die sozio-ökonomische Lage der Befragten wird anhand des letzten Berufsprestiges erfasst. Das Berufsprestige liegt - wie auch bei der Messung des väterlichen Berufspositionierung- in Form der Prestige-Skala nach Treiman (1977) vor $^{46}$ (siehe Fragebogentext und Kodierung 6.9, Punkt c). Um die Dauer der Erwerbstätigkeit zu erfassen, wurden zeitliche Angaben über die verschiedenen Tätigkeitsphasen der Befragten zwischen erster und zweiter Panelwelle zu einer Variablen zusammengefasst. Diese Variable enthält die Dauer der Erwerbstätigkeit in Monaten zwischen 1969/70 und 1984/85 (siehe Fragebogentext und Kodierung 6.9, Punkt d).

Die Beanspruchung durch Kinderbetreuung wird durch eine Variable gemessen, die zählt, bei wie vielen Kindern (inklusive Adoptiv- und Pflegekindern) die der Befragte/ die Befragte die Betreuung im Kleinkindalter übernommen hat (siehe Fragebogentext und Kodierung 6.9 Punkt e).

\footnotetext{
${ }^{46}$ Auf die Verwendung des Einkommen wurde auch hier verzichtet, um die Fallzahlen der Untersuchungsstichprobe nicht weiter zu reduzieren.
} 
Fragebogentext und Kodierung 6.9:

Sekundäre Sozialisationserfahrungen

a) Formales Bildungsniveau im Befragungsjahr 1984/ 85:

\section{Kodierung:}

(1) Maximal Mittlere Reife

(2) Abitur

(3) Studium begonnen, ohne Abschluss

(4) Hochschulabschluss

b) Demonstrationsteilnahme bis $1984 / 85$

- In den letzten Jahren hat es in der Bundesrepublik häufiger öffentliche Demonstrationen gegeben. Haben Sie selbst einmal an einer Demonstration teilgenommen?

\section{Kodierung:}

(0) Nein

(1) Ja

c) Betreuung von Kindern:

- Wenn Kinder vorhanden: Wer hat im wesentlichen die tägliche Versorgung und Pflege von... (Name des Kindes/ der Kinder) als Kleinkind übernommen?

\section{Kodierung:}

(0) Befragte/ $r$ hat keine Betreuung übernommen

(1) Befragte/ $r$ hat bei einem Kind die Betreuung übernommen

(2) Befragte/ $r$ hat bei zwei oder mehr Kindern die Betreuung übernommen

d) Eigenes (letztes) Berufsprestige nach Treiman im J ahr 1984/ 85:

\section{Kodierung:}

(0) 0 bis 40

(1) 41 bis 54

(2) 55 bis 60

(3) 61 bis 78

e) Dauer der Erwerbstätigkeit in Monaten zwischen 1969/ 70 und 1984/ 85:

\section{Kodierung:}

(0) 0 bis 19

(1) 20 bis 46

(2) 47 bis 79

(3) 80 bis 171 


\subsection{Besonderheiten der Untersuchungsstichprobe und Panelmortalität}

Da zu erwarten ist, dass es sich bei der Stichprobe der ehemaligen Gymnasiasten um eine sehr selektive Auswahl handelt, werden im folgenden die sozio-demographischen Verteilungen der Untersuchungsstichprobe (1997) mit einer Substichprobe der ALLBUS-Umfrage aus dem Jahr 1998 verglichen und detaillierter auf die Panelmortalität eingegangen.

\subsubsection{Besonderheiten der Untersuchungsstichprobe}

Für den Stichprobenvergleich wurden aus der ALLBUS-Umfrage des Jahres 1998 alle westdeutschen Befragten im Alter zwischen 41 und 45 Jahren ausgewählt und der Untersuchungsstichprobe - bestehend aus den etwa 43jährigen ehemaligen Gymnasiasten der zweiten Panelwelle gegenübergestellt. Im folgenden werden die Verteilungen der Variablen Geschlecht, Bildung und subjektiver Schichteinschätzung für beide Stichproben aufgeführt (Tabelle 6.1):

Die Gegenüberstellung der beiden Stichproben macht deutlich, dass die Gymnasiastenstichprobe wie vermutet eine sozialstrukturell stark privilegierte Auswahl darstellt. Dieser Befund gilt insbesondere für das Bildungsniveau: Während weniger als $30 \%$ der ALLBUS-Befragten aus der Vergleichsstichprobe über Abitur oder einen höheren Abschluss verfügten, erreichten die ehemaligen Gymnasiasten zu über $80 \%$ die Hochschulreife. Aber auch in der subjektiven Schichteinstufung machen sich erhebliche Unterschiede bemerkbar. So stuften sich rund 30\% der ALLBUS - Befragten in die Unter- oder Arbeiterschicht ein, aber gerade einmal 1,7\% der früheren Gymnasiasten. 
Tabelle 6.1 Zusammensetzung der Untersuchungs- und ALLBUSStichprobe nach sozio-demographischen Merkmalen

\begin{tabular}{|c|c|c|}
\hline & $\begin{array}{c}\text { Ehemalige Gymnasiasten- } \\
\text { Stichprobe } 1997 \\
\% \text { (N) }\end{array}$ & $\begin{array}{c}\text { ALLBUS 1998* } \\
\%(\mathrm{~N}) \\
\end{array}$ \\
\hline \multicolumn{3}{|l|}{ Geschlecht } \\
\hline Männlich & $53,7(857)$ & 55 (99) \\
\hline Weiblich & $46,3(739)$ & $45(81)$ \\
\hline Gesamt & $100(1596)$ & $100(180)$ \\
\hline \multicolumn{3}{|l|}{ Bildung } \\
\hline Ohne Abitur & $16,4(262)$ & $71,1(127)$ \\
\hline \multicolumn{3}{|l|}{ Mit Abitur } \\
\hline Abitur ohne Studium & $7,4(118)$ & \\
\hline Studium ohne Abschluss & $11,4(182)$ & \\
\hline Hochschulabschluss & $57,3(914)$ & \\
\hline Promotion & $7,5(120)$ & \\
\hline Gesamt mit Abitur & $83,6(1334)$ & $28,3(52) * *$ \\
\hline Fehlend & - & $0,6(1)$ \\
\hline Gesamt & $100(1596)$ & $100(180)$ \\
\hline \multicolumn{3}{|l|}{ Subjektive } \\
\hline Unterschicht & $0,2(3)$ & $2,8(5)$ \\
\hline Arbeiterschicht & $1,5(24)$ & $26,1(47)$ \\
\hline Mittelschicht & $56,5(902)$ & $48,9(88)$ \\
\hline Obere Mittelschicht & $37(591)$ & $11,1(20)$ \\
\hline Oberschicht & $2,1(34)$ & $1,7(3)$ \\
\hline Fehlend & $2,6(42)$ & $9,4(17)$ \\
\hline Gesamt & $100(1596)$ & $100(180)$ \\
\hline
\end{tabular}

* Teilstichprobe westdeutsche Befragte im Alter zwischen 41 bis 45 Jahren

** Abschlüsse über Abitur nicht weiter aufgeschlüsselt

Da sowohl das politische Interesse als auch Wertorientierungen von sozialstrukturellen Bedingungen - insbesondere vom Bildungsniveau beeinflusst werden (vgl. Kapitel 4), ist zu vermuten, dass auch bei der Verteilungen der Zielvariablen deutliche Unterschiede zwischen Untersuchungs- und Vergleichsstichprobe bestehen: 
Tabelle 6.2 Verteilung des politischen Interesses und der Wertorientierungen in Untersuchungsund ALLBUS-Stichprobe

\begin{tabular}{|c|c|c|}
\hline & $\begin{array}{c}\text { Ehemalige } \\
\text { Gymnasiasten- } \\
\text { Stichprobe } 1997 \\
\%(\mathrm{~N})\end{array}$ & $\begin{array}{c}\text { ALLBUS 1998* } \\
\%(N)\end{array}$ \\
\hline \multicolumn{3}{|l|}{ Politisches I nteresse } \\
\hline (1) Überhaupt nicht & $1,2(19)$ & $4,5(8)$ \\
\hline (2) Wenig & $10,0(160)$ & $17,8(32)$ \\
\hline (3) Mittel & $41,4(660)$ & $46,7(84)$ \\
\hline (4) Stark & $31,8(508)$ & $23,9(43)$ \\
\hline (5) Sehr stark & $15,5(247)$ & $6,1(11)$ \\
\hline Fehlend & $0,1(2)$ & $1,1(2)$ \\
\hline Gesamt & $100(1596)$ & $100(180)$ \\
\hline Mittelwert & 3,5 & 2,9 \\
\hline Standardabweichung & 0,91 & 0,92 \\
\hline \multicolumn{3}{|l|}{$\begin{array}{l}\text { Wichtigkeit von Politik und } \\
\text { öffentlichem Leben }\end{array}$} \\
\hline 2 & $7(112)$ & $2,8(5)$ \\
\hline 3 & $21(335)$ & $13,3(24)$ \\
\hline 4 & $27,3(435)$ & $34,4(62)$ \\
\hline 5 & $27,8(443)$ & $22,8(41)$ \\
\hline 6 & $11,2(179)$ & $12,2(22)$ \\
\hline 7 =sehr wichtig & $2,8(44)$ & $6,7(12)$ \\
\hline Fehlend & $0,2(3)$ & \\
\hline Gesamt & $100(1596)$ & $100(180)$ \\
\hline Mittelwert & 4,15 & 4,25 \\
\hline Standardabweichung & 1,30 & 1,49 \\
\hline \multicolumn{3}{|l|}{ Inglehart-I ndex } \\
\hline (1) Materialisten & $1,4(23)$ & $13,9(25)$ \\
\hline (2) Materialisten-Mischtyp & $8,4(134)$ & $31,3(56)$ \\
\hline (3) Postmat.-Mischtyp & $21,1(337)$ & $27,2(49)$ \\
\hline (4) Postmaterialisten & $65(1037)$ & $25(45)$ \\
\hline Fehlend & $4,1(65)$ & $2,8(5)$ \\
\hline Gesamt & $100(1596)$ & $100(180)$ \\
\hline Mittelwert & 3,56 & 2,35 \\
\hline Standardabweichung & 0,72 & 1,02 \\
\hline
\end{tabular}

* Teilstichprobe westdeutsche Befragte im Alter zwischen 41 bis 45 J ahren

Wie in Tabelle 6.2 zu sehen ist, liegen sowohl beim politischen Interesse als auch bei den Wertorientierungen erhebliche Differenzen zwischen beiden Stichproben vor. Während 1997 in der Gymnasiasten - Stichprobe 47,3\% der 
Befragten stark oder sehr stark an politischen Angelegenheiten interessiert waren, trifft dies nur auf 30\% der ALLBUS-Befragten zu. Noch stärkere Unterschiede zeigen sich bei den Wertorientierungen. Im Vergleich zur ALLBUS-Auswahl erweist sich die bildungsprivilegierte Untersuchungsstichprobe als außerordentlich postmaterialistisch: 65\% der früheren Gymnasiasten, aber nur 25\% der Vergleichsstichprobe, können als ,reine‘ Postmaterialisten bezeichnet werden.

Die hier beschriebenen Stichprobenbesonderheiten sind bei der Interpretation der Ergebnisse zu berücksichtigen (vgl. Kapitel 8).

\subsubsection{Panelausfälle}

Ein besonderes Problem bei Wiederholungsbefragungen ist der Verlust von Teilnehmern zwischen den verschiedenen Panelwellen, die sogenannte Panelmortalität (vgl. Rendtel 1995, Schnell et al, 1999). Sofern es sich bei der Panelmortalität um neutrale, d.h. zufällige Ausfälle handelt, werden sie abgesehen von der Stichprobenreduktion - als weniger problematisch angesehen.

Handelt es sich jedoch um systematische Ausfälle - indem z. B. die Panelmortalität in einem Zusammenhang mit dem Befragungsthema steht so stellt sich die Frage, inwiefern das Panel noch als repräsentativ für die Grundgesamtheit gelten kann.

Die Ausschöpfungsquoten der hier verwendeten Wiederholungsbefragung (Tabelle 6.3) zeigen, dass die Mehrheit der Ausfälle (38,6\% der Primärbefragung) im Zeitraum von der Primärerhebung bis zur ersten Wiederbefragung zu verzeichnen ist, was bei Panelstudien kein ungewöhnlicher Befund ist (vgl. auch Rendtel 1995: 22). Die Ausschöpfungsquote zwischen der zweiten und dritten Wiederholungsbefragung im Jahr 1996/97 betrug dagegen über 80\% und erreicht damit - insbesondere in Anbetracht des großen Zeitraums zwischen den Panelwellen - eine recht gute Ausschöpfung. Allerdings konnte in der 
Befragungswelle 1996/97 nur noch knapp die Hälfte der ehemaligen Gymnasiasten von 1969/70 befragt werden.

Angesichts der Panelausfälle - insbesondere zwischen den ersten beiden

Tabelle 6.3 Panelausfälle

\begin{tabular}{l|c|c|c|c} 
& $\mathrm{N}$ & $\begin{array}{c}\text { Primär- } \\
\text { stichprobe } \\
\%\end{array}$ & $\begin{array}{c}\text { 1.Wieder- } \\
\text { befragung } \\
\%\end{array}$ & $\begin{array}{c}2 . \text { Wieder- } \\
\text { befragung } \\
\%\end{array}$ \\
\hline $\begin{array}{l}\text { 1. Primärbefragung 1969/70 } \\
\text { (Nettostichprobe) } \\
\text { = Bruttostichprobe für 1. }\end{array}$ & 3.240 & 100 & & \\
$\begin{array}{l}\text { Wiederbefragung 84/85 } \\
\text { 2. Erste Wiederbefragung 1984/85 } \\
=\text { Bruttostichprobe für 2. }\end{array}$ & 1.987 & 61,4 & 100 & \\
Wiederbefragung 96/97 & & & & \\
\hline 3. Zweite Wiederbefragung & 1.596 & 49,3 & 80,2 & 100 \\
\hline
\end{tabular}

Quelle: ZA-Information 42, S. 132, eigene Zusammenstellung

Panelwellen - stellt sich die Frage nach den Ursachen der Panelmortalität bzw. ob und inwieweit mit Verzerrungen hinsichtlich der abhängigen Variablen zu rechnen ist.

Analysen zu den Ursachen der Panelausfälle wurden jeweils im Anschluss an die Wiederholungsbefragungen von den Primärforschern selbst erstellt (vgl. Meulemann et al. 1987, Birkelbach 1998).

Es stellte sich dabei heraus, dass - verglichen mit der Ausgangsstichprobe bei der ersten Wiederbefragung Befragte ohne Abitur um 2,8 Prozentpunkte unterrepräsentiert sind (Meulemann et al. 1987: 85f.). Die Unterrepräsentation dieser Gruppe verstärkte sich bei der zweiten Wiederbefragung noch einmal um weitere 0,8 Prozentpunkte. Die Tatsache, dass Befragte ohne Hochschulreife überproportional seltener zur Teilnahme an der ersten Wiederbefragung bewegt werden konnten, führen die Autoren auf das etwas unglücklich formulierte Anschreiben zurück, mit dem die ehemaligen Schüler vor der Wiederholungsbefragung kontaktiert wurden. In diesem Anschreiben wurde die Studie mit dem Titel "Lebensplanung und Lebenserfolg” erläutert und dabei mehrmals der Begriff „Erfolg“ verwendet. Rückblickend kommen die Verfasser zu dem Schluss, dass eine derart normativ besetzte Formulierung die Gefahr in sich birgt, dass insbesondere 
diejenigen ehemaligen Gymnasiasten, die ihre Schullaufbahn ohne Abitur verließen, befürchten mussten, als ,erfolglos' dazustehen. Demnach zeigte sich die Tendenz, dass die ,Erfolgreicheren' eher bereit waren, über ihren bisherigen Lebensverlauf Auskunft zu geben (Meulemann et al. 1987, Birkelbach 1998: 18).

In Bezug auf auf sozio-demographische Merkmale wie Alter, Geschlecht oder Konfession liegen den Autoren zufolge keine größeren Einschränkungen der Repräsentativität vor (Meulemann et al. 1987: 87f.).

Zu den Ursachen der Panelmortalität zwischen der ersten und zweiten Wiederbefragung (1985 und 1997) gehören in erster Linie die Erreichbarkeit der Befragungsperson, die allgemeine Kooperationsbereitschaft, das Interesse am Thema, die themenbezogene Selbsteinstufung und Einschätzung der sozialen Wertschätzung dieser Position (in der Befragung: Erfolg im Lebenslauf) sowie die Einschätzung eigener kognitiver Kompetenz bezüglich des Befragungsgegenstandes (Birkelbach 1998: 143).

Um abschätzen zu können, ob die beobachteten Panelausfälle in der vorliegenden Arbeit zu Verzerrungen hinsichtlich der Zielvariablen führen, werden abschließend die Teilnehmer aller Befragungswellen mit denjenigen Personen verglichen, die in der letzten Panelwelle nicht mehr befragt werden konnten (vgl. Tabelle 6.4) ${ }^{47}$.

47 Da ein Zusammenhang zwischen den Zielvariablen und dem Bildungsniveau der Befragten vermutlich auch in der vorliegenden Stichprobe besteht und Befragte mit niedrigerem Bildungsniveau überdurchschnittlich häufig eine Wiederbefragung verweigerten, ist bei den abhängigen Variablen mit Verzerrungen zu rechnen (vgl. auch Kapitel zu den Besonderheiten der Stichprobe, siehe Kapitel 6.4.1). 
Tabelle 6.4 Vergleich zwischen Panelausfällen und der Untersuchungsstichprobe von 1997

\begin{tabular}{c|c|c|c|c} 
& $\begin{array}{c}\mathbf{1 9 8 5} \text { und } \\
\mathbf{1 9 9 7} \\
\text { befragt }\end{array}$ & $\begin{array}{c}\text { Panelausfälle } \\
\text { zwischen 1985 } \\
\text { und 1997 }\end{array}$ & $\begin{array}{c}\text { Mittel- } \\
\text { wert- } \\
\text { Differenz }\end{array}$ & Eta \\
\hline $\begin{array}{c}\text { Politisches I nteresse 1985 } \\
\text { Mittelwert }\end{array}$ & 3,49 & 3,33 & $-0,16^{* *}$ & 0,069 \\
\hline Standardabweichung & 0,93 & 0,92 & & \\
\hline N & 1588 & 387 & & \\
\hline $\begin{array}{c}\text { Wichtigkeit von Politik und } \\
\text { offentlichem Leben 1985 }\end{array}$ & & & $-0,27^{*}$ & 0,073 \\
\hline Mittelwert & 4,60 & 4,33 & \\
\hline Standardabweichung & 1,41 & 1,54 & & \\
\hline N & 1588 & 384 & & \\
\hline $\begin{array}{c}\text { Inglehart-I ndex 1985 } \\
\text { Mittelwert }\end{array}$ & 3,57 & 3,45 & $-0,12^{*}$ & 0,063 \\
\hline Standardabweichung & 0,74 & 1,02 & & \\
\hline N & 1564 & 369 & & \\
\hline
\end{tabular}

* signifikant bei einer Irrtumswahrscheinlichkeit von $<5 \%$

$* *$ signifikant bei einer Irrtumswahrscheinlichkeit von $<1 \%$

Wie in Tabelle $6.4 \mathrm{zu}$ sehen ist, erreichen diejenigen, die an allen Befragungswellen teilnahmen, bei allen drei Zielvariablen im Jahr 1985 im Schnitt etwas höhere Werte als die Befragten, die nach 1985 aus dem Panel ausschieden. Die jeweiligen Mittelwertdifferenzen sind in allen drei Fällen signifikant, die entsprechenden Eta-Koeffizienten sprechen aber nicht für substanzielle Zusammenhänge zwischen beiden Variablen. Somit lässt sich festhalten, dass zwischen der zweiten und dritten Panelwelle nicht mit größeren Verzerrungen bei den abhängigen Variablen zu rechnen ist.

Ob dieser Befund auch für die Panelmortalität zwischen erster und zweiter Panelwelle gilt, kann jedoch nicht abgeschätzt werden, da die abhängigen Variablen in der Erstbefragung nicht erhoben wurden (siehe Fußnote vorherige Seite).

Insgesamt weist vor allem der Befund, dass überproportional viele Befragte ohne Hochschulreife die Teilnahme verweigerten, darauf hin, dass die Wiederholungsbefragungen 1984/85 und 1996/97 keine reine Zufallsstichproben der zuvor befragten Gruppe sind. Somit können Verzerrungen bei den statistischen Tests nicht ausgeschlossen werden, was bei der Bewertung der Ergebnisse zu berücksichtigen ist. 


\section{5 Übersicht über die verwendeten Variablen und Konstrukte}

Tabelle 6.5 Variablen zur Sozialisation

\begin{tabular}{|c|c|}
\hline Konstrukt & I ndikator \\
\hline SES Jugendzeit & $\begin{array}{l}\text { Berufsprestige des Vaters nach Treiman } \\
\text { Schulbildung Vater } \\
\text { Schulbildung Mutter }\end{array}$ \\
\hline $\begin{array}{l}\text { Kognitive Kompetenz } \\
\text { in der J ugend }\end{array}$ & $\begin{array}{l}\text { Analogie-Test nach Amthauer } \\
\text { Wortauswahl-Test nach Amthauer }\end{array}$ \\
\hline Geschlecht & Geschlecht \\
\hline $\begin{array}{l}\text { Bewertung Familien- } \\
\text { klima in Jugendzeit }\end{array}$ & $\begin{array}{l}\text { Spannungen zwischen Eltern und Kindern im } \\
\text { Elternhaus } \\
\text { Kindererziehung wie bei den Eltern }\end{array}$ \\
\hline $\begin{array}{l}\text { Erziehungsziel } \\
\text { selbständiges Denken } \\
\text { und Handeln }\end{array}$ & $\begin{array}{l}\text { Von Eltern gewünschte Erziehungsziele der } \\
\text { Schule: } \\
\text { Erziehungsziel gesellschaftliche Kritikfähigkeit } \\
\text { Erziehungsziel selbständige Problemlösung }\end{array}$ \\
\hline $\begin{array}{l}\text { Protesterfahrungen } \\
\text { in der Schule }\end{array}$ & Häufigkeit Protesterfahrungen in der Schule \\
\hline $\begin{array}{l}\text { Kontakt mit } \\
\text { Sozialwissenschaften } \\
\text { im Schulkontext }\end{array}$ & $\begin{array}{l}\text { Beliebteste vs. unbeliebteste Fächer aus dem } \\
\text { sozialwissenschaftlichen Bereich } \\
\text { Durchschnittsnote in sozialwissenschaftlichen } \\
\text { Fächern } \\
\text { Begabung in sozialwissenschaftlichen Fächern } \\
\text { (Elternmeinung) }\end{array}$ \\
\hline $\begin{array}{l}\text { Image Politik in der } \\
\text { Schulklasse }\end{array}$ & $\begin{array}{l}\text { Wahrgenommene Bewertung von politischem } \\
\text { Engagement in der Schulklasse }\end{array}$ \\
\hline $\begin{array}{l}\text { Politische Partizipation in } \\
\text { Jugendzeit a) }\end{array}$ & $\begin{array}{l}\text { Mitglied in politischer Schülergruppe oder in } \\
\text { politischer Gruppe } 1969 / 70\end{array}$ \\
\hline $\begin{array}{l}\text { Politische Partizipation in } \\
\text { Jugendzeit b) }\end{array}$ & Demonstrationsteilnahme 1969/1970 \\
\hline $\begin{array}{l}\text { Wunsch nach früher } \\
\text { Partizipationsmöglichkeit }\end{array}$ & $\begin{array}{l}\text { Gewünschtes Alter für Möglichkeit politischer } \\
\text { Betätigung }\end{array}$ \\
\hline Bildung bis 1985 & Formales Bildungsniveau \\
\hline $\begin{array}{l}\text { Partizipationserfahrung bis } \\
1985\end{array}$ & Teilnahme an Demonstration \\
\hline $\begin{array}{l}\text { Beanspruchung durch } \\
\text { Kinderbetreuung }\end{array}$ & Häufigkeit der Kleinkindbetreuung \\
\hline $\begin{array}{l}\text { Eigene sozio-ökonomische } \\
\text { Stellung }\end{array}$ & Letztes Berufsprestige nach Treiman \\
\hline $\begin{array}{l}\text { Beanspruchung durch } \\
\text { Berufstätigkeit }\end{array}$ & Dauer der Erwerbstätigkeit \\
\hline
\end{tabular}




\section{$7 \quad$ Ergebnisse}

\subsection{Stabilität der Zielvariablen}

Nach Jagodzinski und Kühnel (1987: 226) lassen sich drei Quellen für Veränderungen unterscheiden. Die erste Quelle kann als identische Verschiebung aller Werte, gemessen über $a$, beschrieben werden. Perfekte Stabilität liegt bei $a=0$ vor:

$$
\eta_{t+1, j}=a+\eta_{t, j}
$$

Im zweiten Fall wird das Ausmaß der Stabilität durch den Veränderungsfaktor $\beta$ gemessen. Perfekte Stabilität besteht hier bei $\beta=1$ :

$$
\eta_{t+1, j}=\beta^{*} \eta_{t, j}
$$

Die dritte Möglichkeit nimmt an, dass die Instabilität durch externe Veränderungsschocks, gemessen über die Höhe der Varianz v, ausgelöst wird:

$$
\eta_{t+1, j}=\eta_{t j}+v_{t+1, j}
$$

Im folgenden wird als Maß für die Stabilität $r$ bzw. $r^{2}$ betrachtet. Inhaltlich bedeutet dies, dass die Verschiebung $a \neq 0$ und multiplikative Veränderung $\beta$ $\neq 1$ auf die Messung der Stabilität keine Auswirkungen haben und somit in erster Linie die dritte Quelle von Instabilität betrachtet wird.

Eine Ausnahme stellen die Interaktionshypothesen dar, bei denen vermutet wird, dass unterschiedliche Veränderungsfaktoren $(\beta)$ vorliegen. Daher wird hier zusätzlich das unstandardisierte Regressionsgewicht $\beta$ betrachtet.

Vor der Untersuchung der individuellen Stabilität der Zielvariablen werden im folgenden zunächst die Verteilungen und deskriptiven Kennwerte der Indikatoren für beide Befragungszeitpunkte auf der Aggregatebene beschrieben. 


\subsubsection{Politisches Interesse 1985-1997}

Zur Messung des politischen Interesses stehen in dem verwendeten Datensatz zwei Indikatoren zur Verfügung: das subjektive politische Interesse und die Variable ,Wichtigkeit von Politik und öffentlichem Leben'. Diese beiden Variablen werden zunächst getrennt untersucht. Auf diese Weise lässt sich ermitteln, ob die Entwicklung beider Indikatoren in der Untersuchungskohorte nach einem ähnlichen Muster vonstatten geht, oder ob sie substanzielle Unterschiede aufweist. Wenn in der zeitlichen Entwicklung Unterschiede in der Stabilität feststellbar sind, dann weist dies darauf hin, dass beide Variablen verschiedene Sachverhalte messen. Sind etwaige Stabilitätsdifferenzen deutlich ausgeprägt, so ist die Verwendung dieser Indikatoren zur multiplen Messung des politischen Interesses nicht adäquat.

\subsubsection{Die Randverteilungen von ,subjektivem politischen I nteresse' und ,Wichtigkeit von Politik' 1985-1997}

Die Querschnittverteilungen für die Entwicklung des subjektiven Politikinteresses offenbaren für die Untersuchungsstichprobe ein insgesamt recht starkes, subjektives politisches Interesse (vgl. Tabelle 7.1): Jeweils knapp die Hälfte der Befragten berichtet zu beiden Zeitpunkten ein starkes Interesse an Politik. Betrachtet man die Entwicklung der Verteilungen zwischen beiden Panelwellen, so zeigt sich eine sehr große Aggregatstabilität. Der Anteil der stark Interessierten (bei Zusammenfassung der Kategorien ,stark' und ,sehr stark') verändert sich beispielsweise nur leicht von 48,3\% im Jahr 1985 auf 47,4\% im Jahr 1997.

Gänzlich unverändert bleiben die Mittelwerte der Verteilungen zwischen beiden Befragungsjahren. 
Im Vergleich zum subjektiven Politikinteresse ist der Indikator, Wichtigkeit

Tabelle 7.1 Subjektives politisches Interesse, 1985-1997 (Angaben in Prozent, in Klammern: Fallzahlen)

\begin{tabular}{l|l|l|l}
\hline & \multicolumn{2}{|c|}{ Subjektives politisches Interesse } & \\
\hline Ausprägung & 1985 & 1997 & $\begin{array}{c}\text { Mittelwertdifferenz } \\
1985-1997\end{array}$ \\
\hline Sehr Schwach & $1.5(23)$ & $1.2(19)$ & \\
\hline Schwach & $11.3(179)$ & $10.0(160)$ & \\
Mittel & $39.0(618)$ & $41.4(660)$ & \\
\hline Stark & $33.6(533)$ & $31.9(508)$ & \\
\hline Sehr Stark & $14.7(233)$ & $15.5(247)$ & \\
\hline Gesamt & $100 *(1586)$ & $100(1594)$ & 0.00 \\
\hline MW & 2.36 & 2.36 & \\
\hline SD & 0.70 & 0.68 & \\
\hline
\end{tabular}

*Abweichungen von 100 gehen auf Rundungen zurück

von Politik' zwischen den beiden Untersuchungszeitpunkten weniger stabil (Tabelle

7.2). Bei diesem Indikator zeigt sich auf der Aggregatebene eine sichtbare Verschiebung: Während im Jahr 1985 über die Hälfte der Befragten (55,8\% bei Zusammenfassung der Kategorien 5-7) eine hohe Wichtigkeit von Politik angaben, lag der Anteil 1997 bei nur noch 41,7\%. Dass die Bedeutung von Politik in der Kohorte der ehemaligen Gymnasiasten zwischen beiden Untersuchungszeitpunkten abnimmt, sieht man auch beim Mittelwertvergleich: Zwischen 1985 und 1997 nahm der Mittelwert des Indikators höchst signifikant um 0,29 Skalenpunkte ab.

\footnotetext{
${ }^{48}$ Berechnet mit dem T-Test für gepaarte Stichproben.
} 
Tabelle 7.2: Wichtigkeit von Politik, 1985-1997

(Angaben in Prozent, in Klammern: Fallzahlen)

\begin{tabular}{l|l|l|l}
\hline & \multicolumn{2}{|c|}{ Wichtigkeit von Politik } & \\
\hline Ausprägung & 1985 & 1997 & $\begin{array}{c}\text { Mittelwertdifferenz } \\
1985-1997\end{array}$ \\
\hline (1) Sehr Schwach & $2.3(35)$ & $0(0)$ & \\
\cline { 1 - 2 }$(2)$ & $4.3(66)$ & $7.2(112)$ & \\
\cline { 1 - 2 }$(3)$ & $14.5(223)$ & $21.6(335)$ & \\
\cline { 1 - 2 }$(4)$ & $23.2(358)$ & $28.1(435)$ & \\
\hline$(6)$ & $27.7(427)$ & $28.6(443)$ & $-0.39 * * *$ \\
\hline (7) Sehr Stark & $19.3(298)$ & $11.6(179)$ & \\
\hline Mesamt & $8.8(136)$ & $2.8(44)$ & \\
\hline SD & $100 *(1543)$ & $100 *(1548)$ & \\
\hline
\end{tabular}

*Abweichungen von 100 gehen auf Rundungen zurück 


\subsubsection{Zur individuellen Stabilität des politischen Interesses 1985-1997}

Die individuelle Stabilität der Indikatoren für das politische Interesse lässt sich durch die Analyse der Aggregatveränderungen nicht ermitteln. Auf der Aggregatebene zeigen die Veränderungen lediglich die Summe aller individuellen Bewegungen in der Stichprobe und können - überträgt man sie auf die Individualebene - zu erheblichen Fehlschlüssen führen. Ein stabiles Niveau auf der Aggregatebene kann zwar daraus resultieren, dass sich die individuellen Angaben der Befragten zwischen beiden Zeitpunkten tatsächlich nicht verändert haben. Es kann aber auch dann zustande kommen, wenn die Werte eines Teils der Befragen ansteigen, während sie bei einem anderen Teil um den gleichen Betrag sinken. In der Summe bliebe in einem solchen Fall der Aggregatwert ebenfalls unverändert.

Um festzustellen, wie stark sich das subjektive Politikinteresse und die "Wichtigkeit von Politik" tatsächlich verändert hat, werden im Folgenden die individuellen Bewegungen der Befragten zwischen beiden Panelwellen betrachtet (Tabelle 7.3 und 7.4). 
Tabelle 7.3 Individuelle Stabilität des subjektiven politischen

I nteresses, 1985-1997

(Angaben in Prozent, in Klammern: Fallzahlen)

\begin{tabular}{|c|c|c|c|c|c|c|}
\hline \multirow[b]{2}{*}{$\begin{array}{l}\text { Subjektives } \\
\text { politisches } \\
\text { Interesse } 1997\end{array}$} & \multicolumn{6}{|c|}{ Subjektives politisches Interesse 1985} \\
\hline & $\begin{array}{l}\text { Sehr } \\
\text { Schwach }\end{array}$ & Schwach & Mittel & Stark & $\begin{array}{l}\text { Sehr } \\
\text { Stark }\end{array}$ & Gesamt \\
\hline Sehr Schwach & $21.7(5)$ & $2,8(5)$ & $0,8(5)$ & $0,8(4)$ & $0,0(0)$ & $1,2(19)$ \\
\hline Schwach & $39.1(9)$ & $34,1(61)$ & $12,0(74)$ & $2,4(13)$ & $1,3(3)$ & $10,1(160)$ \\
\hline Mittel & $34.8(8)$ & $51,4(92)$ & $\begin{array}{l}57,3 \\
(354)\end{array}$ & $\begin{array}{l}33,0 \\
(176)\end{array}$ & $11,2(26)$ & $41,4(656)$ \\
\hline Stark & $4.3(1)$ & $8,9(16)$ & $\begin{array}{l}24,6 \\
(152)\end{array}$ & $\begin{array}{l}50,3 \\
(268)\end{array}$ & $29,2(68)$ & $31,8(505)$ \\
\hline Sehr Stark & $0.0(0)$ & $2.8(5)$ & $5.3(33)$ & $13.5(72)$ & $\begin{array}{l}58.4 \\
(136)\end{array}$ & $15.5(246)$ \\
\hline Gesamt & $\begin{array}{l}100 * \\
(23) \\
\end{array}$ & $\begin{array}{l}100 \\
(179) \\
\end{array}$ & $\begin{array}{l}100 \\
(618) \\
\end{array}$ & $\begin{array}{l}100 \\
(533) \\
\end{array}$ & $\begin{array}{l}100 * \\
(233) \\
\end{array}$ & $100(1586)$ \\
\hline Stabil & \multicolumn{6}{|c|}{51.9 (824) Summe der Diagonale } \\
\hline Abnahme & \multicolumn{6}{|c|}{23.6 (374) Summe oberhalb der Diagonale } \\
\hline Zunahme & \multicolumn{6}{|c|}{24.5 (388) Summe unterhalb der Diagonale } \\
\hline $\begin{array}{l}\text { Polychorische } \\
\text { Korrelation }\end{array}$ & \multicolumn{6}{|l|}{0.629} \\
\hline $\begin{array}{l}\text { Produkt-Moment- } \\
\text { Korrelation }\end{array}$ & \multicolumn{6}{|l|}{0.564} \\
\hline
\end{tabular}

*Abweichungen von 100 gehen auf Rundungen zurück

Die Untersuchung der individuellen Veränderungen zeigt bei beiden Indikatoren eine deutlich geringere Stabilität als die Aggregatwerte vermuten lassen. Betrachtet man die Stabilitätsraten für das subjektive politische Interesse im Panel, so bleibt der Wert bei kapp 52\% der Befragten über 13 Jahre hinweg stabil. Jeweils ungefähr ein Viertel der Befragten äußert in der zweiten Wiederbefragung im Vergleich zur ersten Panelwelle ein höheres oder aber ein geringeres subjektives Politikinteresse ${ }^{49}$.

\footnotetext{
49 Allerdings wird hier ein strenges Kriterium für Stabilität angelegt. Fasst man die Kategorien sehr schwach und schwach (bzw. "stark" und "sehr stark") zusammen, betrachtet also einen Wechsel zwischen schwach und sehr schwach (bzw. "stark" und "sehr stark") nicht als Instabilität, dann erhöht sich die Stabilität auf $62 \%$.
} 
Ein weiterer Befund der Kreuztabellierung ergibt sich für die Stabilität des subjektiven Interesses in Abhängigkeit von der Stärke des Politinteresses. Es zeigt sich, dass die Stabilität des Politikinteresses der schwach Interessierten im Vergleich zu derjenigen der mittel bis stark Interessierten erheblich geringer ist ${ }^{50}$. Dieser Aspekt wird in Kapitel 7.2 ausführlicher behandelt.

Betrachtet man die Korrelation zwischen beiden Zeitpunkten als Maß für die Stabilität der Indikatoren (polychorische Korrelation bzw. Pearson's r. Tabelle 7.3 und Tabelle 7.4), so zeigt sich für das subjektive politische Interesse bei der Berücksichtigung des ordinalen Skalenniveaus ein polychorischer Korrelationskoeffizient von 0.629 (bzw. eine Produktmomentkorrelation von 0.564).

Angesichts des langen Befragungsintervalls von 13 Jahren kann die Stabilität des subjektiven Politikinteresses damit als recht hoch beschrieben werden.

Im Vergleich zum subjektiven Politikinteresse ist die Stabilität der individuellen Wichtigkeit von Politik unter den Befragten deutlich geringer (Tabelle 7.3 und 7.4). Legt man liberalere Kriterien an die Definition von Stabilität an, indem man etwa einen Wechsel innerhalb der oberen und der unteren drei Kategorien nicht als Instabilität betrachtet und die Werte zusammenfasst, so bleibt etwa die Hälfte der Panelteilnehmer (48.31\%) in ihrer Bewertung der Bedeutung der Politik stabil. Auffällig ist zudem, dass von den verbleibenden knapp 62\% der Befragten der weitaus größere Teil die Wichtigkeit von Politik 1997 geringer einstuft als noch 1985 (33.4\% von allen Befragten). Nur für 18.4\% hingegen nimmt in den Jahren zwischen 1985 und 1997 die Bedeutung von Politik zu.

\footnotetext{
50 Jedoch ist zu bemerken, dass die Kategorien der schwach Interessierten erheblich weniger Fälle enthalten.
} 
Tabelle 7.4 Individuelle Stabilität der Wichtigkeit von Politik, 1985-1997

(Angaben in Prozent, in Klammern: Fallzahlen)

\begin{tabular}{l|l|l|l|l}
\hline & \multicolumn{3}{|c}{ Wichtigkeit von Politik 1985 } & Gesamt \\
\hline Wichtigkeit von Politik 1997 & Schwach & Mittel & Stark & \\
\hline Schwach (Werte 1-3) & $49.7(161)$ & $33.8(121)$ & $18.9(162)$ & $28.8(444)$ \\
\hline Mittel (Wert 4) & $25.6(83)$ & $33.0(118)$ & $26.9(231)$ & $28.1(432)$ \\
\hline Stark (Werte 5-7) & $24.7(80)$ & $33.2(119)$ & $54.2(465)$ & $43.1(664)$ \\
\hline Gesamt & $100(324)$ & $100(358)$ & $100(858)$ & $100(1540)$ \\
\hline Stabil & $48.31 \%(744)$ Summe der Diagonale & \\
\hline Abnahme & $33.38 \% \quad$ (514) Summe oberhalb der Diagonale \\
\hline Zunahme & $18.31 \% \quad$ (282) Summe unterhalb der Diagonale \\
\hline Polychorische Korrelation & 0.378 & \\
\hline Produkt-Moment-Korrelation & 0.356 & \\
\hline
\end{tabular}

Berechnet man die Stabilität der Angaben nicht auf der Basis der trichotomisierten Variablen, sondern der Orginalskalierung ${ }^{51}$, so steigt die Instabilität stark an: Nur 27.2\% der Werte erweisen sich als stabil. 1997 hält dagegen fast die Hälfte der Befragten (47.1\%) Politik in ihrem Leben für weniger wichtig als noch im Jahr 1985. Für das restliche Viertel steigt die Bedeutung von Politik in dem betreffenden Zeitraum.

Dass die Variable ,Wichtigkeit von Politik', verglichen mit dem subjektiven Politikinteresse, deutlich instabiler ist, wird auch bei der Berechnung des Stabilitätskoeffizienten sichtbar: Die Autokorrelation zwischen 1985 und 1997 liegt bei diesem Indikator nur 0.378 (bzw. $r=0.356$. vgl. Tabelle 7.4). Dieser Wert entspricht einem Anteil von gerade einmal $12.7 \%$ erklärter Varianz gegenüber $31.8 \%$ gebundener Varianz für das subjektive politische Interesse.

\footnotetext{
${ }^{51}$ D.h. eine Instabilität liegt bereits bei einer Veränderung von einem Punkt auf der 7er-Skala vor.
} 
Die deskriptiven Befunde zu den beiden Indikatoren, die als multiple Indikatoren zur Messung des Politikinteresses vorgesehen waren, machen deutlich, dass die These van Deth's (2000), wonach die beiden Variablen analytisch zu trennen seien (vgl. Kapitel 6), durchaus ihre Berechtigung hat.

Die Konsequenz für die vorliegende Arbeit besteht darin, dass für beide Indikatoren getrennte Pfadmodelle berechnet werden. D.h. es wird kein Modell mit multiplen Indikatoren berechnet, sondern ein Single-IndikatorModell mit dem subjektiven politischen Interesse (s. Kapitel 7.2). 


\subsubsection{Wertorientierungen 1985-1997}

Als dritte Zielvariable wird Verteilung und Entwicklung der gesellschaftspolitischen Wertorientierungen nach Inglehart betrachtet.

\subsubsection{Die Randverteilungen der Wertorientierungen 1985-1997}

Die Aggregatverteilung der politischen Wertorientierungen dokumentiert, dass sowohl 1985 als auch 1997 postmaterialistische Ziele in der vorliegenden Stichprobe eine bemerkenswert hohe Zustimmung finden (Tabelle 7.5): Jeweils mehr als zwei Drittel der Befragten können Ingleharts Vorstellungen folgend als reine „Postmaterialisten" eingestuft werden $(70.3 \%$ bzw. 67.7\%). "Reine Materialisten", aber auch die sogenannten „materialistischen Mischtypen" (vgl. Kapitel 6), stellen in der Kohorte der ehemaligen Gymnasiasten dagegen nur eine Minderheit dar. Das starke Übergewicht der Postmaterialisten in der Stichprobe - insbesondere im Vergleich zur ALLBUS-Auswahl (vgl. Kapitel 6) - deutet auf einen möglicherweise starken Bildungseffekt hin.

Tabelle 7.5 Postmaterialismus, 1985-1997

(Angaben in Prozent, in Klammern: Fallzahlen)

\begin{tabular}{l|l|l|l}
\hline & \multicolumn{2}{|c|}{ Postmaterialismus } & \\
\hline Ausprägung & 1985 & 1997 & \multirow{2}{*}{ Mittelwertdifferenz } \\
\hline Materialisten & $1.9(28)$ & $1.5(23)$ & \\
\cline { 1 - 2 } Mat-Mischtypen & $10.1(152)$ & $8.8(134)$ & \\
\cline { 1 - 3 } Pmat-Mischtypen & $17.7(266)$ & $22.0(337)$ & \\
\hline Postmaterialisten & $70.3(1057)$ & $67.7(1037)$ & \\
\hline Gesamt & $100(1503)$ & $100(1531)$ & \\
\hline MW & 3.56 & 3.56 & 0.00 \\
\hline SD & 0.75 & 0.71 & \\
\hline
\end{tabular}


Insgesamt weist die Untersuchungsstichprobe im Aggregat recht stabile Wertprioritäten auf. Ein leichter Rückgang ist bei den reinen postmaterialistischen Wertvorstellungen zu verzeichnen (minus 2.6\%). Demgegenüber steigt zwischen 1985 und 1997 der Anteil der postmaterialistischen Mischtypen leicht an (plus 4.3\%). Die Mittelwerte der Verteilungen bleiben zwischen beiden Befragungsjahren bei einem Wert von 3.56 unverändert.

\subsubsection{Zur individuellen Stabilität der Wertorientierungen 1985-1997}

Die Werte für die individuelle Stabilität der Wertorientierungen sind der Tabelle 7.6 zu entnehmen. Die Zahlen belegen eine recht hohe Stabilität des Indikators über die Zeitspanne: Bei nicht ganz zwei Drittel der Befragten finden zwischen dem 30. und 43. Lebensjahr keine Veränderungen der Wertvorstellungen statt.

Von den restlichen knapp 35\% verändert die eine Hälfte ihre Wertprioritäten in Richtung Materialismus (17.5\%), die andere Hälfte denkt 1997 stärker postmaterialisch als 13 Jahre zuvor (17.3\%). 
Die hohe individuelle Wertestabilität ist - wie ein Blick auf Tabelle 7.6 verrät vor allem auf die Konstanz der postmaterialistischen Zielvorstellungen zurückzuführen: Fast $80 \%$ derjenigen, die 1985 als Postmaterialisten eingestuft wurden, verändern zwischen beiden Befragungszeitpunkten ihre Wertorientierungen nicht. Materialisten, aber auch die beiden Mischtypen, weisen hingegen sehr viel instabilere Wertorientierungen auf (Stabilitätswerte von $14.3 \%$ bis $36.5 \%$ ). Die Stabilitätskoeffizienten (Polychorische Korrelation und Produkt-Moment-Korrelation) liegen mit 0.557 und $0.416 \mathrm{im}$ mittleren bis hohen Bereich.

Tabelle 7.6 I ndividuelle Stabilität von Postmaterialismus, 1985-1997

(Angaben in Prozent, in Klammern: Fallzahlen)

\begin{tabular}{|c|c|c|c|c|c|}
\hline & \multicolumn{5}{|c|}{ Postmaterialismus 1985} \\
\hline $\begin{array}{l}\text { Postmaterialismus } \\
1997\end{array}$ & $\begin{array}{l}\text { Materi- } \\
\text { alisten }\end{array}$ & $\begin{array}{l}\text { Mat- } \\
\text { Mischtype } \\
n\end{array}$ & $\begin{array}{l}\text { Pmat- } \\
\text { Mischtype } \\
n\end{array}$ & $\begin{array}{l}\text { Postmateri- } \\
\text { alisten }\end{array}$ & Gesamt \\
\hline Materialisten & $14.3(4)$ & $3.3(5)$ & $2.6(7)$ & $.6(6)$ & $1.5(22)$ \\
\hline Mat-Mischtypen & $35.7(10)$ & $23.0(35)$ & $14.3(38)$ & $4.5(48)$ & $8.7(131)$ \\
\hline Pmat-Mischtypen & $32.1(9)$ & $44.1(67)$ & $36.5(97)$ & $15.0(159)$ & $22.1(332)$ \\
\hline Postmaterialisten & $17.9(5)$ & $29.6(45)$ & $46.6(124)$ & $79.8(844)$ & $67.7(1018)$ \\
\hline Gesamt & $100(28)$ & $100(152)$ & $100(266)$ & $100(1057)$ & $100(1503)$ \\
\hline Stabil & \multicolumn{5}{|c|}{65.20 (980) Summe der Diagonale } \\
\hline Abnahme & \multicolumn{5}{|c|}{17.50 (263) Summe oberhalb der Diagonale } \\
\hline Zunahme & \multicolumn{5}{|c|}{17.30 (260) Summe unterhalb der Diagonale } \\
\hline $\begin{array}{l}\text { Polychorische } \\
\text { Korrelation }\end{array}$ & \multicolumn{5}{|l|}{0.557} \\
\hline $\begin{array}{l}\text { Produkt-Moment- } \\
\text { Korrelation }\end{array}$ & \multicolumn{5}{|l|}{0.416} \\
\hline
\end{tabular}




\section{2 Überprüfung der Kausalhypothesen}

\subsubsection{Statistische Vorgehensweise}

In diesem Abschnitt werden zunächst die gewählten Analyseverfahren für die Hypothesenüberprüfung dargestellt. Anschließend erfolgt die Präsentation der Ergebnisse.

Für die in Kapitel 5 vermuteten Zusammenhänge können neben direkten auch indirekte Effekte auf die Zielvariablen erwartet werden. Indirekte Einflüsse sollten dabei insbesondere von den sozio-demographischen Hintergrundvariablen wie Herkunft und Geschlecht ausgehen. Aus diesem Grund ist es erforderlich, in der statistischen Datenanalyse komplexe Mehrvariablenmodelle zu verwenden, die neben der Modellierung direkter Einflusspfade auch Aussagen zu indirekten Effekten zulassen.

Ein solches Vorgehen erlaubt das Verfahren der Pfadanalyse. Sie verbindet mehrere Regressionsgleichungen miteinander und kann als Modifikation der multivariaten Regressionsanalyse betrachtet werden (vgl. Kühnel/Krebs 2007: 601, Opp/Schmidt 1976: 311f.). Mit der Pfadanalyse ist es möglich, mehrere abhängige Variablen zu formulieren, die gleichzeitig als erklärende Einflussgrößen für andere Variablen fungieren. In der Terminologie der Pfadanalyse werden alle Variablen, die in einer Modellgleichung erklärt werden, als endogene Variablen bezeichnet. Exogene Variablen hingegen sind solche Merkmale, die im Modell durch keine anderen Variablen beeinflusst werden. Mit der Einbeziehung mehrerer sowohl exogener als auch endogener Variablen ist die Pfadanalyse anders als die ,einfache' Regressionsanalyse in der Lage, simultan direkte und indirekte Effekte zwischen den jeweiligen Variablen zu überprüfen. Durch Addition der direkten und indirekten Effekte lässt sich zusätzlich der sogenannte totale Effekt einer erklärenden auf eine endogene Variable ermitteln.

Während in der Pfadanalyse die Überprüfung der Variablenbeziehungen über einzelne manifeste Variablen geschieht, verwenden Strukturgleichungsmodelle mit latenten Variablen multiple Indikatoren zur Erfassung der theoretischen Konstrukte (vgl. Bollen 1989: 11, Reinecke 2005: 99). Mit Hilfe eines solchen multiplen Indikatorenmodells versucht 
man, die Fehlerhaftigkeit einer Messung insgesamt zu reduzieren, indem man verschiedene Indikatoren zur gemeinsamen Messungen einer latenten Variablen heranzieht.

Im Idealfall erfasst das Strukturgleichungsmodell - bzw. die konfirmatorische Faktorenanalyse - möglichst viele der in der Fragestellung formulierten theoretischen Konstrukte mit einer möglichst großen Zahl an geeigneten Indikatoren.

Für die hier vorliegende Fragestellung ließen sich leider nur für vier theoretische Konzepte mehrere akzeptable Indikatoren in den verwendeten Datensätzen finden (siehe Übersicht zu den Konstrukten, Tabellen 6.13. und 6.14). Die verbleibenden theoretischen Konstrukte bzw. latenten Variablen werden daher mit den gemessenen manifesten Variablen gleichgesetzt. In diesen Fällen wird somit auf eine Messfehlerkorrektur verzichtet.

Zur Überprüfung der kausalen Beziehungen zwischen den latenten Variablen innerhalb der Pfadmodelle stehen mehrere Strategien zur Verfügung. Nach Jöreskog (1993) lassen sich drei Strategien der Modellprüfungen unterscheiden: In der ,strictly confirmatory situation' wird die Übereinstimmung eines theoretisch abgeleiteten Modells mit den empirischen Daten geprüft. Die Ergebnisse der Berechnung führen zur Annahme oder Ablehnung des Modells. In der "Alternative Models Situation" werden hingegen mehrere Modelle mit dem Ziel getestet, das „beste" Modell zu identifizieren. Bei der dritten Strategie, der ,Model Generating Situation' wird versucht, ein Modell zu finden, das sowohl gut zu den Daten passt als auch theoretisch begründet ist. Dabei werden - ausgehend von den Prüfergebnissen eines ersten theoretisch begründeten Ausgangsmodells sukzessive weitere Modellversionen geprüft, bis ein zufriedenstellendes Modell identifiziert ist.

Für die Testung der hier spezifizierten Kausalmodelle (siehe Abbildung 7.1 und 7.2) wird die letzte der drei Vorgehensweisen, die ,Model Generating Situation', angewendet. Mit dieser Strategie verlässt man allerdings den Weg einer konfirmatorischen Modellprüfung zugunsten einer eher explorativ ausgerichteten Datenanalyse. 
In Bezug auf den Begriff der Kausalität ist darauf hinzuweisen, dass Kausalität im eigentlichen Sinne hier nicht geprüft werden kann. Zwar werden in den Sozialwissenschaften Pfad- oder Strukturgleichungsmodelle häufig auch als Kausalmodelle bezeichnet. Mit dem Verfahren der Pfadanalyse kann jedoch nur geprüft werden, ob das postulierte Kausalmodell statistisch nicht zurückzuweisen ist (vgl. Bortz 2005:480, Reinecke 2005:12). Gelingt eine "gute" Anpassung des Modells an die empirischen Daten, so heißt dies lediglich, dass die dem Modell zugrundeliegenden Hypothesen gut mit den Daten übereinstimmen, dass sie also nicht widerlegt wurden. Eine solche Übereinstimmung könnte aber auch für die Modellierung einer alternativen Kausalstruktur gelten.

Für die Berechnung von Pfad- bzw. Strukturgleichungsmodellen mit dem üblicherweise verwendeten Maximum Likelihood (ML)-Verfahren wird vorausgesetzt, dass die beobachteten Variablen auf metrischem Skalenniveau gemessen werden und sowohl kontinuierlich als auch multivariat normalverteilt sind. Diese Bedingungen sind bei der Analyse von Umfragedaten allerdings nur sehr selten erfüllt.

Da die vorliegenden Daten teilweise sehr schief verteilt sind, wird hier für die Berechnung der Pfadmodelle auf das Weigthed Least Squares (WLS)Verfahren mit listwise deletion zurückgegriffen ${ }^{1}$. Ein wesentlicher Vorteil der WLS-Schätzung besteht darin, dass bei einer hinreichend großen Stichprobe unabhängig von der Verteilung der verwendeten Indikatoren - asymptotisch korrekte Schätzungen für Parameter, Standardfehler und Anpassungsmaße ermittelt werden (vgl. Bollen: 425f. 1989: Reinecke 2005: 112).

Um das überwiegend ordinale Messniveau der Daten berücksichtigen zu können, werden zur Berechnung der Kausalmodelle darüber hinaus

\footnotetext{
${ }^{1}$ Zur Behandlung der fehlenden Werte wurde das Verfahren ,listwise deletion' gewählt, da die Methode WLS bei ,pairwise deletion' nicht verwendet werden kann und andere Verfahren mit erheblichen Nachteilen - etwa Varianzreduktion oder Verzerrungen - verbunden sind (Vgl. Reinecke 2005: 283f.).
} 
polychorische Varianzen und Kovarianzen verwendet ${ }^{2}$. Der Schätzung polychorischer Varianzen und Kovarianzen liegt die Annahme zugrunde, dass es sich bei ordinalen Variablen um ungenaue Messungen unbeobachteter, intervallskalierter latenter Variablen handelt. Die polychorische Korrelation kann somit als eine Schätzung der Produktmomentkorrelation bezeichnet werden (vgl. Reinecke 2005: 257, Bollen 1989: 441f.) ${ }^{3}$.

Insgesamt lässt sich festhalten, dass die WLS-Schätzmethode mit der Verwendung polychorischer Varianzen und Kovarianzen das für die Analyse der vorliegenden Daten angemessenste Verfahren darstellt. Die gewählte Vorgehensweise sollte am ehesten in der Lage sein, eventuelle Zusammenhänge zwischen exogenen und endogenen Variablen zu entdecken.

\subsubsection{Spezifikation des Analysemodells für das politische Interesse}

In diesem Abschnitt erfolgt zunächst die Überprüfung des nach den Hypothesen AF1 bis AS4 abgeleiteten Kausalmodells für das politische Interesse (vgl. Abbildung 7.1). Die Überprüfung der in den Hypothesen AI1 und AI2 formulierten Interaktionseffekte wird anschließend dargestellt. Das Kapitel 7.2.3 behandelt die Überprüfung des Kausalmodells für die Wertorientierungen.

In einer ersten bivariaten Analyse werden den Hypothesen entsprechend die einfachen Zusammenhänge zwischen den erklärenden Variablen und der abhängigen Variable Politikinteresse untersucht. Dazu wurden die polychorischen Korrelationen berechnet (s. Tabelle 7.7):

\footnotetext{
2 Die polychorischen Koeffizienten lassen sich auch beim Vorliegen dichotomer Variablen schätzen (vgl. Reinecke 2005:35) Die Schwellenwerte zwischen den Messwiederholungen wurden bei der Berechnung der polychorische Varianzen und Kovarianzen gleichgesetzt.

3 Dass polychorische Korrelationen verglichen mit anderen Assoziationsmaßen die beste Schätzung für die "wahren Korrelationen" liefern, konnte mit Hilfe von Simulationsstudien gezeigt werden. Dabei wurden kontinuierliche Variablen, deren ,wahre' Korrelation bekannt
} 
Tabelle 7.7: Polychorische Korrelationen der Indikatoren mit "Subjektives politisches Interesse“ basierend auf der Untersuchungsstichprobe von max. 1596

\begin{tabular}{l|l}
\hline \multicolumn{1}{c|}{ Indikatoren } & $\begin{array}{c}\text { Subjektives politisches } \\
\text { I nteresse 1985 }\end{array}$ \\
\hline Treiman-Prestigeskala Vater & 0.047 \\
\hline Schulbildung Vater & 0.001 \\
\hline Schulbildung Mutter & 0.015 \\
\hline Analogie-Test nach Amthauer & 0.117 \\
\hline Wortauswahl-Test nach Amthauer & 0.056 \\
\hline Geschlecht & $-0.357^{*}$ \\
\hline Spannungen zwischen Eltern und Kindern im Elternhaus & -0.122 \\
\hline Kindererziehung wie bei den Eltern & -0.131 \\
\hline Protesterfahrung in der Schule & 0.071 \\
\hline Beliebteste vs. unbeliebteste Fächer aus dem & $0.246^{*}$ \\
sozialwissenschaftlichen Bereich & 0.201 \\
\hline Durchschnittsnote in sozialwissenschaftlichen Fächern & $0.245^{*}$ \\
\hline Begabung in sozialwissenschaftlichen Fächern & 0.169 \\
\hline Wahrgenommenes Image von Politik in der Schulklasse & $-0.109 *$ \\
\hline Gewünschtes Partizipationsalter & 0.141 \\
\hline Partizipationserfahrung bis 1969 & $0.122^{*}$ \\
(Teilnahme Demonstration) & $0.393^{*}$ \\
\hline Mitglied in politischer Gruppe & $0.220^{*}$ \\
\hline Partizipationserfahrung bis 1985 & -0.211 \\
\hline Bildung bis 1985 & 0.020 \\
\hline Betreuung Kleinkinder bis 1985 & \\
\hline Berufsprestige bis 1985 & \\
\hline
\end{tabular}

* Signifikant auf dem 5\%-Niveau

Wie in Tabelle $7.7 \mathrm{zu}$ sehen ist, bestätigt die einfache Korrelationsanalyse bis auf eine Ausnahme - die theoretisch erwarteten Zusammenhänge. Allerdings weisen einige der Koeffizienten nur sehr geringe Werte auf bzw. sind insignifikant.

Nicht hypothesenkonform sind dagegen die Vorzeichen für die Korrelationen zwischen dem Politikinteresse und den Indikatoren zur Messung des Familienklimas. Diese - allerdings nur sehr schwach ausgeprägten negativen Koeffizienten besagen, dass das politische Interesse im 30.

war, in ordinale Variablen umgewandelt und anschließend untersucht (Jöreskog/Sörbom 1988). 
Lebensjahr um so schwächer ausgeprägt ist, je positiver die 15-jährigen Jugendlichen 1969/70 das Familienklima wahrgenommenen haben (siehe dazu auch weiter unten).

Um ein übersichtliches Modell zu schätzen, gehen in die Berechnung des Ausgangspfadmodells für das politische Interesse - abgesehen von den Variablen zur Messung des sozio-ökonomischen Status, der kognitiven Kompetenz und des Familienklimas - nur solche Variablen ein, die auf der bivariaten Ebene einen Korrelationskoeffizienten von mindestens $0.15^{4}$ aufweisen. Dabei handelt es sich um folgende Variablen (bzw. Konstrukte):

- Geschlecht (GESCHL) ${ }^{5}$

- Sozio-ökonomischer Status in der Jugendzeit (SES)

- Kognitive Kompetenz in der Jugendzeit (KOGKOMP)

- Bewertung des Familienklimas in der Jugendzeit (KLIMA FAMILIE)

- Kontakt mit Sozialwissenschaften im Schulkontext (SOWI SCHULE)

- Wahrgenommenes Image von Politik in der Schulklasse (IMAGE POLITIK SCHULE)

- Erreichter Bildungsabschluss bis 1985 (BILDUNG 1985)

- Partizipationserfahrung bis 1985 (PARTIZIP 1985)

- Beanspruchung durch Kinderbetreuung bis 1985 (KINDER 1985)

Im ersten Schritt der Kausalanalyse werden die Faktorladungen jener Konstrukte überprüft, für die mehrere Indikatoren zur Messung vorliegen (s. Tabelle 7.8, vgl. auch polint ausgangsmodell.pdf).

\footnotetext{
${ }^{4}$ Auch in einer zusätzlich durchgeführten multiplen Regressionsrechnung ergaben sich keine weiteren Variablen, die einen relevanten signifikanten Einfluss auf die Zielvariable aufweisen. ${ }^{5}$ In den Klammen sind die Kurzbezeichnungen für die Konstrukte aufgeführt.
} 
Tabelle 7.8: Standardisierte Faktorladungen der latenten Konstrukte

\begin{tabular}{|c|c|c|c|c|}
\hline Indikatoren & SES & $\begin{array}{l}\text { KOG- } \\
\text { KOMMP }\end{array}$ & $\begin{array}{l}\text { KLI MA } \\
\text { FAMI LI E }\end{array}$ & $\begin{array}{l}\text { SOWI } \\
\text { SCHULE }\end{array}$ \\
\hline Berufsprestige des Vaters & 0.68 & & & \\
\hline Schulbildung Vater & 0.75 & & & \\
\hline Schulbildung Mutter & 0.95 & & & \\
\hline Analogie & & 0.65 & & \\
\hline Wortauswahl & & 0.40 & & \\
\hline $\begin{array}{l}\text { Spannungen zwischen Eltern und } \\
\text { Kindern im Elternhaus }\end{array}$ & & & 0.66 & \\
\hline Kindererziehung wie bei den Eltern & & & 0.78 & \\
\hline $\begin{array}{l}\text { Beliebteste vs. unbeliebteste Fächer } \\
\text { aus dem sozialwissenschaftlichen } \\
\text { Bereich }\end{array}$ & & & & 0.59 \\
\hline $\begin{array}{l}\text { Durchschnittsnote in } \\
\text { sozialwissenschaftlichen Fächern }\end{array}$ & & & & 0.70 \\
\hline $\begin{array}{l}\text { Begabung in sozialwissenschaftlichen } \\
\text { Fächern }\end{array}$ & & & & 0.80 \\
\hline
\end{tabular}

Die standardisierten Faktorladungen für drei der vier Konstrukte können als zufriedenstellend bewertet werden. Die Faktorladungen für das Konstrukt ,kognitive Kompetenz' sind hingegen nicht als ausreichend anzusehen. Hier erreicht nur der Indikator ,Analogie' einen zufriedenstellenden Wert. Der Wert für den Indikator Wortauswahl ist dagegen unbefriedigend; das gemeinsame Konstrukt ,kognitive Kompetenz' vermag hier gerade einmal 16\% der Varianz dieses Indikators zu erklären. Dieser Indikator wird dennoch verwendet, da sonst für dieses Konstrukt keine Messfehlerkorrektur vorgenommen werden könnte. 
Das zur Überprüfung der Hypothesen spezifizierte Ausgangsmodell ist in Abbildung 7.1 wiedergegeben.

Abbildung 7.1: Ausgangsmodell zur Erklärung des politischen Interesses

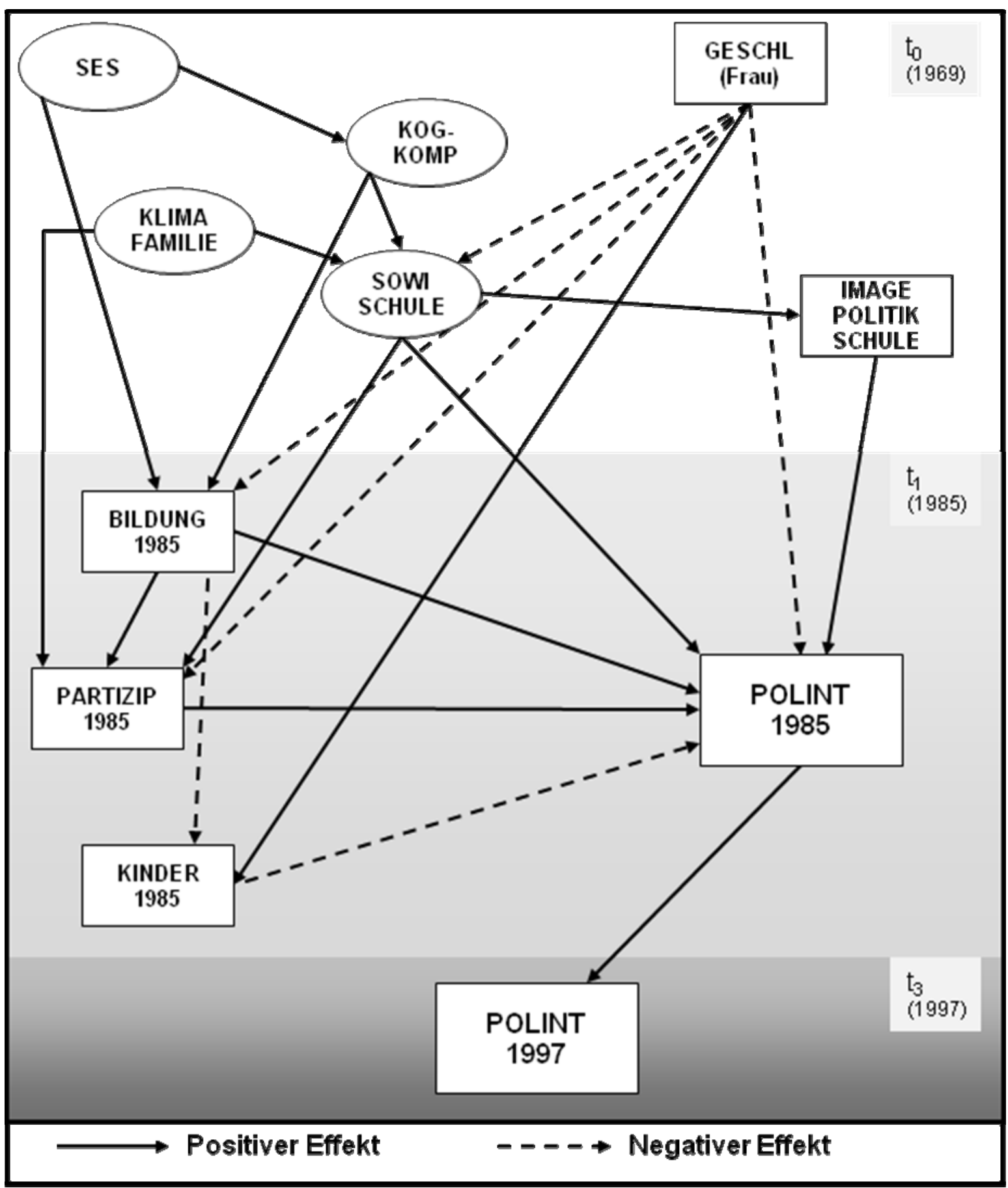


Die Werte für die Modellanpassung des Nullmodells können der Tabelle 7.9 entnommen werden ${ }^{6}$. Die in der Tabelle aufgeführten Anpassungswerte nach dem Chi-Ouadrat-Anpassungstest zeigen keine gute Modellanpassung für das Ausgangsmodell. Dies bedeutet, dass zwischen den modellimplizierten und empirischen Varianzen und Kovarianzen deutliche Abweichungen bestehen und die zugrundeliegenden Annahmen nicht mit den Daten vereinbar sind.

Tabelle 7.9: Modellanpassung für das Ausgangsmodell

\begin{tabular}{c|c|c|c|c|c}
\hline $\begin{array}{c}\text { Chi- } \\
\text { Quadrat }\end{array}$ & Df & P & RMSEA & p-Close & N \\
\hline 321.41 & 105 & 0.000 & 0.042 & 0.99 & 1153 \\
\hline
\end{tabular}

Die Tatsache, dass das Ausgangsmodell keinen guten Modellfit aufweist, ist für ein Ausgangsmodell allerdings kein ungewöhnlicher Befund. Hinzu kommt, dass bei hohen Fallzahlen aufgrund der größeren Teststärke bereits kleine Abweichungen zwischen den modellimplizierten und empirischen Varianzen und Kovarianzen zu einer Ablehnung des Modells führen. Daher wird in der Literatur oft empfohlen, die Chi-Quadrat-Statistik nicht als inferenzstatistisches, sondern als deskriptives Kriterium für die Modellprüfung zu verwenden (vgl. Reinecke 2005: 216).

Als Maß für die Modellanpassung wird daher der sogenannte RMSEA (,Root Mean Square Error of Approximation') verwendet. RMSEA basiert auf der sogenannten ,Populationsdiskrepanzfunktion' (PDF), setzt diese aber ins Verhältnis zu den Freiheitsgeraden. Er hat eine Untergrenze von Null, wenn das Modell genau zu den Daten passt. RMSEA-Werte von 0.08 werden im Allgemeinen als große Diskrepanzen gewertet. Werte zwischen 0.05 und 0.08 lassen sich als mittlere und Werte kleiner als 0.05 als kleine Diskrepanzen interpretieren. Der Modellfit kann bei Werten unter 0.05 als gut angesehen

\footnotetext{
${ }^{6}$ Die Berechnungen der Strukturgleichungsmodelle wurden mit LISREL 8.72 von Karl Jöreskog und Dag Sörbom von durchgeführt.
} 
werden. Zusätzlich wird der p-close-Wert angegeben, der einen Wert von über 0.5 aufweisen sollte (vgl. Reinecke: 120) ${ }^{7}$.

Dem modellgenerierenden Vorgehen entsprechend wird das Ausgangsmodell nun sukzessive modifiziert und optimiert. Ein wichtiges Werkzeug zur Modellmodifikation ist der sogenannte Modifikationsindex. Dieser Index wird in Lisrel für jeden restringierten Wert berechnet und gibt an, mit welcher Änderung des $X^{2}$ Wertes zu rechnen ist, wenn der betreffende Parameter freigesetzt wird (Bollen 1989: 305).

Um die Modellanpassung zu verbessern werden - den Modifikationsindizes folgend - schrittweise Parameter freigesetzt, sofern dies mit inhaltlichen Gesichtpunkten vereinbar ist ${ }^{8}$. Beendet wird die Modellanpassung dann, wenn sich mit weiteren Veränderungen bei den Pfaden zwischen den latenten Variablen keine relevante Modellverbesserung mehr ergibt (Modifikationsindizes < 7).

Im Vergleich zum Ausgangsmodell hat sich die Modellanpassung beim Endmodell deutlich verbessert (Tabelle 7.10) ${ }^{9}$.

Tabelle 7.10: Modellanpassung für das modifizierte Modell, Endmodell

\begin{tabular}{c|c|c|c|c|c}
\hline $\begin{array}{c}\text { Chi- } \\
\text { Quadrat }\end{array}$ & Df & p & RMSEA & p-Close & N \\
\hline 180.50 & 104 & 0.000 & 0.025 & 1 & 1153 \\
\hline
\end{tabular}

${ }^{7}$ Ein p-close-Wert testet die Nullhypothese, dass der RMSEA-Populationswert nicht kleiner ist als 0.05 .

8 Die jeweiligen Zwischenschritte werden hier nicht im Detail wiedergegeben. Die Modifikationen können der Datei ,polint endmodell.pdf' entnommen werden, die auf der beiliegenden $C D$ dokumentiert ist.

9 Eine weitere Verbesserung des Modellfits ließe sich erreichen, wenn Nebenladungen und Fehlerkorrelationen zugelassen werden. Eine probeweise durchgeführte Modellmodifikation durch Freigabe von Nebenladungen und Meßfehlerkovarianzen ergab, dass die Parameterwerte im Hinblick auf die endogenen Variablen ,Politisches Interesse 1985' und ,Politisches Interesse 1997' stabil bleiben. Da diese Nebenladungen und Messfehlerkorrelationen jedoch nur in Ausnahmefällen inhaltlich interpretiert werden können, wird auf die Freigabe der Parameter hier verzichtet. 
Das modifizierte Strukturmodell für das politische Interesse ist der Abbildung 7.2 zu entnehmen, die Beta-Koeffizienten sind in Tabelle 7.11 wiedergegeben ${ }^{10}$.

\section{Abbildung 7.2: Endmodell zur Erklärung des politischen Interesses}

10 Alle Koeffizienten sind mindestens auf dem 5\%-Niveau signifikant. Der Übersichtlichkeit wegen wird hier -wie auch bei den folgenden Tabellen- auf die Angabe der Standardfehler verzichtet. Sie sind in den entsprechenden Dateien auf der beiliegenden CD dokumentiert. 


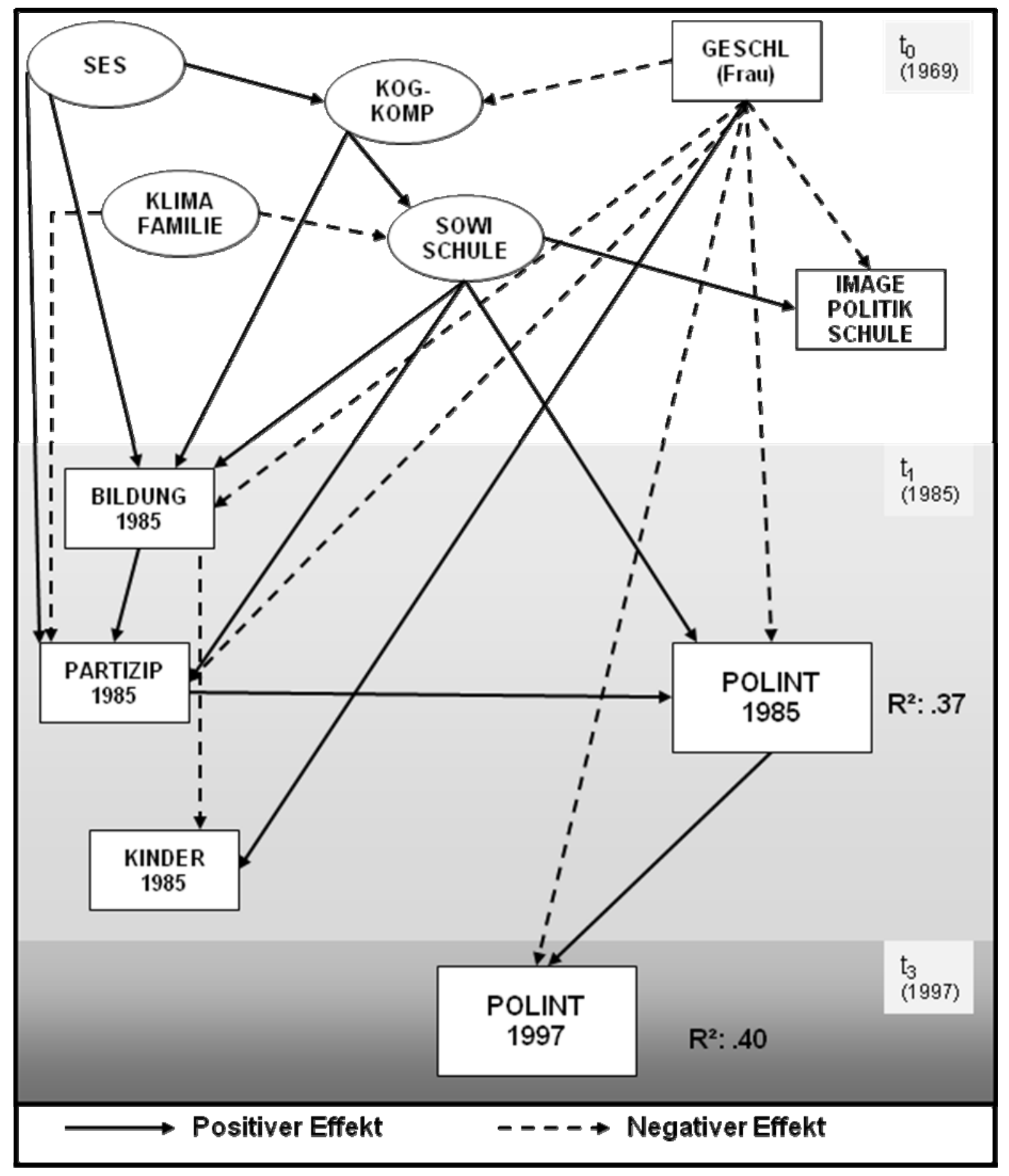




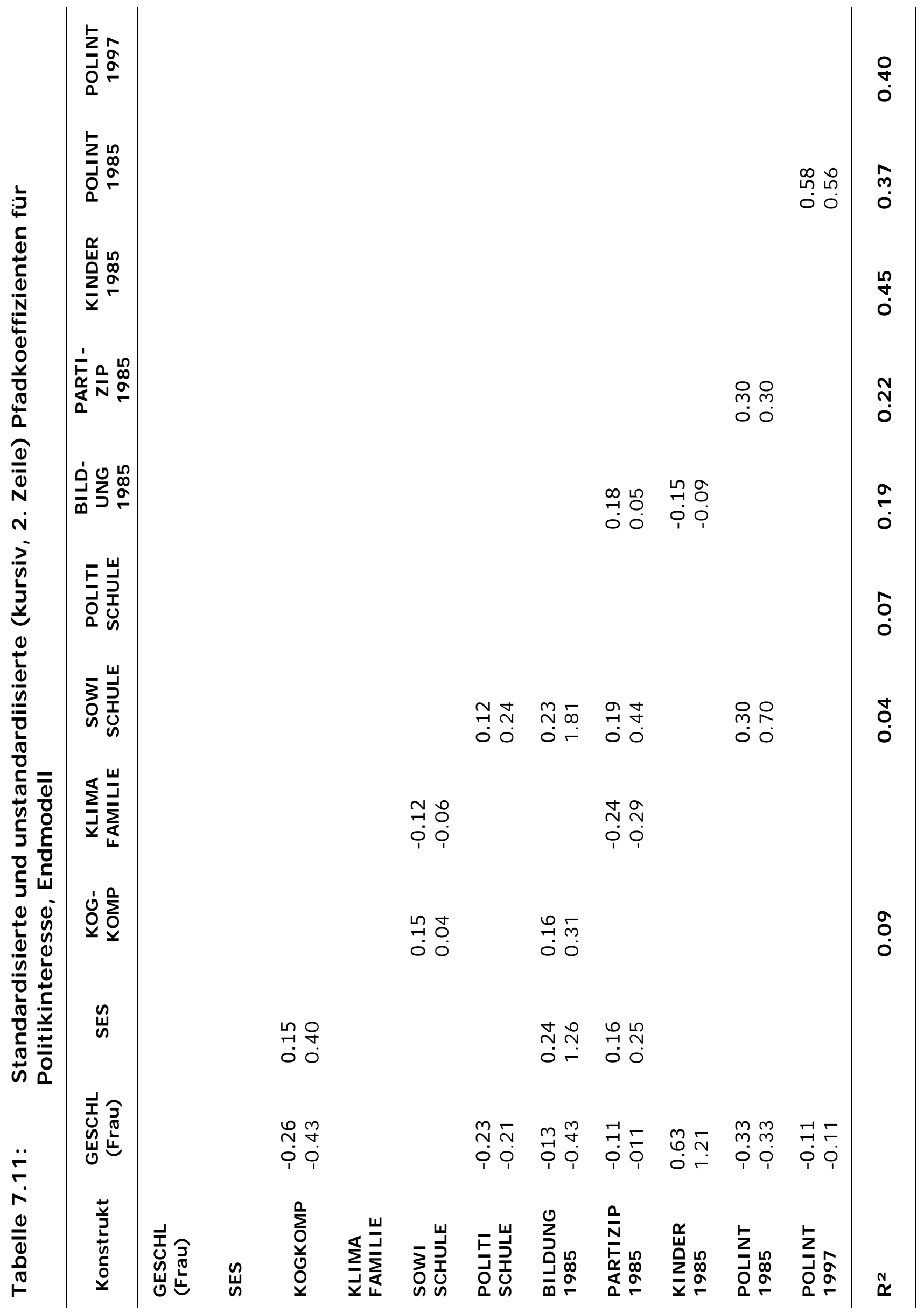


Als besonders bedeutsam für die Erklärung von politischem Interesse erweist sich der Faktor Geschlecht. Dieser Befund ist jedoch nicht weiter überraschend, sondern steht im Einklang mit dem in Kapitel 4 referierten Forschungsstand. Der Faktor ,Sozio-ökonomische Stellung des Elternhaus' (SES) spielt dagegen nur eine untergeordnete Rolle. Sein Einfluss ist eher als gering einzustufen und verläuft indirekt - vor allem über Bildung und eigene politische Partizipationserfahrungen vermittelt (siehe auch Tabelle 7.12).

Die ,jugendliche kognitive Kompetenz' (KOGKOMP) wirkt ebenfalls indirekt und relativ schwach, hat aber den erwarteten positiven Einfluss auf das spätere Politikinteresse.

\section{Tabelle 7.12: Totale und indirekte Effekte auf das Politikinteresse 1985: Standardisierte Effekte (in Klammern unstandardisiert)}

Totale Effekte*

\begin{tabular}{l|c}
\hline Konstrukt & Polint85 \\
\hline Geschlecht & $0.39(-0.39)$ \\
\hline SES Jugend & $0.07(0.11)$ \\
\hline Kognitive Kompetenz Jugend & $0.04(0.04)$ \\
\hline Familienklima & $-0.12(-0.14)$ \\
\hline SOWI Schule & $0.37(0.86)$ \\
\hline Image Politik Schule & - \\
\hline Bildung bis 1985 & $0.05(0.02)$ \\
\hline Partizipation bis 1985 & $0.30(0.30)$ \\
\hline Kinder bis 1985 & - \\
\hline
\end{tabular}

Indirekte Effekte*

\begin{tabular}{|c|c|}
\hline Konstrukt & Polint85 \\
\hline Geschlecht & $-0.06(-0.06)$ \\
\hline SES Jugend & $0.07(0.11)$ \\
\hline Kognitive Kompetenz Jugend & $0.06(0.04)$ \\
\hline Familienklima & $-0.12(-0.15)$ \\
\hline SOWI Schule & $0.07(0.16)$ \\
\hline Image Politik Schule & - \\
\hline Bildung bis 1985 & $0.05(0.02)$ \\
\hline Partizipation bis 1985 & - \\
\hline Kinder bis 1985 & - \\
\hline
\end{tabular}

Der insgesamt stärkste Effekt auf das Politikinteresse im 30. Lebensjahr geht neben dem Geschlecht von der manifesten politischen Sozialisation in der Schule aus - gemessen über das Konstrukt, SOWI SCHULE'. Der 
standardisierte totale Effekt von 0.37 macht deutlich, dass die manifeste politische Sozialisation in der Jugend einen erheblichen Einfluss auf das spätere politische Interesse hat: je mehr die Jugendlichen in der mittleren Adoleszenz mit sozialwissenschaftlichen Inhalten im Unterricht in Kontakt kommen, umso stärker ist ihr Politikinteresse im späteren Erwachsenenalter. Das Familienklima weist überraschenderweise einen, zwar nur leichten, aber negativen Einfluss auf. Eine positive oder harmonische Atmosphäre im Elternhaus fördert nicht das Politikinteresse, sondern reduziert es im Gegenteil.

Keine Bedeutung für das politische Interesse hat dagegen das zweite Konstrukt zur Messung jugendlicher Sozialisationserfahrungen in der Schule, die wahrgenommene Politisierung der Schulklasse.

Als kaum relevant erweist sich auch das Bildungsniveau: lediglich ein indirekter Effekt von 0.05, der über die politische Partizipation verläuft, ist hier zu vermerken. Die geringe Effektstärke der Bildung ist vermutlich durch die geringe Variation der Bildung in der selektiven Stichprobe der ehemaligen Gymnasiasten begründet. Die Tatsache, dass in der vorliegenden Stichprobe das Politikinteresse im Vergleich zur ALLBUS-Stichprobe deutlich stärker ausgeprägt ist, kann aber an sich schon als Beleg für Bildungseinflüsse gewertet werden.

Ein deutlicher Einfluss auf das Politikinteresse im 30. Lebensjahr geht von der eigenen politischen Partizipation nach der mittleren Jugendzeit aus: Befragte, die bis zum zweiten Erhebungszeitpunkt an einer (oder mehreren) politischen Demonstration(en) teilgenommen haben, sind wesentlich stärker an politischen Angelegenheiten interessiert, als solche, die nicht demonstriert haben. Allerdings lässt sich hier die kausale Struktur des Wirkmechanismus nicht eindeutig klären, da beide Variablen zum zweiten Befragungszeitpunkt erhoben wurden. Anders als bei der manifesten politischen Sozialisation in der Adoleszenz kann hier nicht entschieden werden, ob tatsächlich die politische Partizipation politisierend wirkt, ob politisch Interessierte häufiger an Demonstrationen teilnehmen oder ob möglicherweise wechselseitige Einflüsse vorliegen.

Für das politische Interesse ist die Belastung, die von Kinderbetreuung ausgeht, völlig unbedeutend. Die in der bivariaten Analyse gemessene 
Korrelation stellt sich als Scheinkausalität dar, die sich fast vollständig durch das weibliche Geschlecht der Betreuungspersonen erklären lässt.

Insgesamt liegt die Erklärungskraft des Strukturmodells bei einem $R^{2}$ von 0.37 für das Politikinteresse im 30. Lebensjahr. Für ein Pfadmodell, in dem die abhängige Variable mit nur einem Indikator gemessen wird, ist dies ein recht zufriedenstellender Wert.

Abschließend stellt sich die Frage nach dem relativen Einfluss der verschiedenen Faktoren für das Politikinteresse im 30. Lebensjahr. Insgesamt ist hierzu festzustellen, dass in dem spezifizierten Kausalmodell die früheren Sozialisationseinflüsse alles in allem stärker wirksam sind als jene Erfahrungen, die nach der mittleren Jugendzeit gesammelt werden. Dies gilt insbesondere für die manifeste politische Sozialisation (in der Schule) und für das Geschlecht, das für eine herausragende Bedeutung geschlechtspezifischer Sozialisation steht.

\subsubsection{Geschlechtsspezifische Unterschiede bei der Erklärung des Politikinteresses}

Die Hypothese, dass die Kausalstruktur zur Erklärung des Politikinteresses geschlechtsspezifische Unterschiede aufweist, soll nun mit dem Verfahren des multiplen Gruppenvergleichs untersucht werden. Mit diesem Vergleich kann getestet werden, ob die Struktur des Kausalmodells über die Geschlechtsgruppen hinweg variiert. Auch bei der Berechnung der multiplen Gruppenvergleiche wird schrittweise vorgegangen: Das Ausgangsmodell überprüft, ob sich sämtliche Parameter über beide Gruppen hinweg gleichsetzen lassen. Wird die Nullhypothese verworfen, so wird sukzessiv überprüft, ob sich das Modell verbessert, wenn einzelne Parameter zur Schätzung freigegeben werden.

In den folgenden beiden Tabellen 7.13 und 7.14 sind die Ergebnisse für den Gruppenvergleich zwischen Männern und Frauen dargestellt. Die Tabelle 7.13 gibt die Modellanpassung für das Ausgangs- und Endmodell wieder: 
Tabelle 7.13 Gruppenvergleich zwischen Männern und Frauen, Modellanpassung für das Ausgangs- und Endmodell ${ }^{11}$

\begin{tabular}{l|l|l|l|l|l}
\hline Modell & $\begin{array}{l}\text { Chi- } \\
\text { Quadrat }\end{array}$ & Df & p & RMSEA & p-Close \\
\hline Ausgangsmodell & 1294.04 & 230 & 0.000 & 0.090 & 0.000 \\
\hline Endmodell & 308.57 & 204 & 0.000 & 0.030 & 1 \\
\hline
\end{tabular}

In der Tabelle 7.14 sind die unstandardisierten Pfadkoeffizienten des Endmodells ausgewiesen. Signifikante Unterschiede zwischen Männer und Frauen werden besonders hervorgehoben (fett und unterstrichen).

Die jeweiligen modifizierten Strukturmodelle für beide Geschlechter sind in den Abbildungen 7.3a und 7.4b wiedergegeben.

${ }^{11}$ Die Faktorladungen im Endmodell sind gleichgesetzt. Die Messfehler- und Faktor sowie die Betakoeffizienten konnten z.T. gleichgesetzt werden, in anderen Fällen wurden sie frei geschätzt. Die Parameter des Endmodells können der Datei ,polintgrup endmodell.pdf auf der beiliegenden $C D$ entnommen werden. 


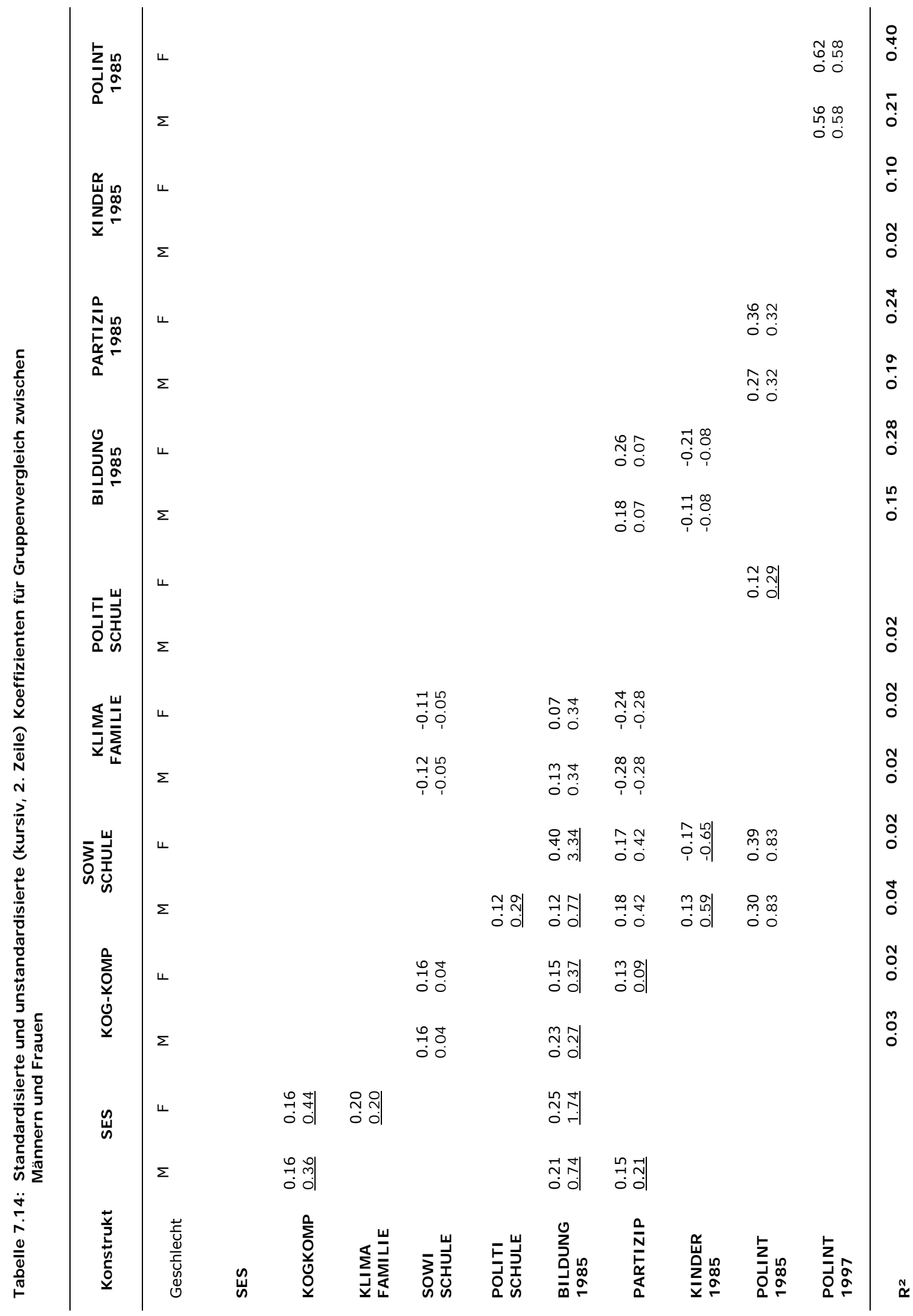


Abbildung 7.3a: Endmodell zur Erklärung des politischen I nteresses bei Frauen

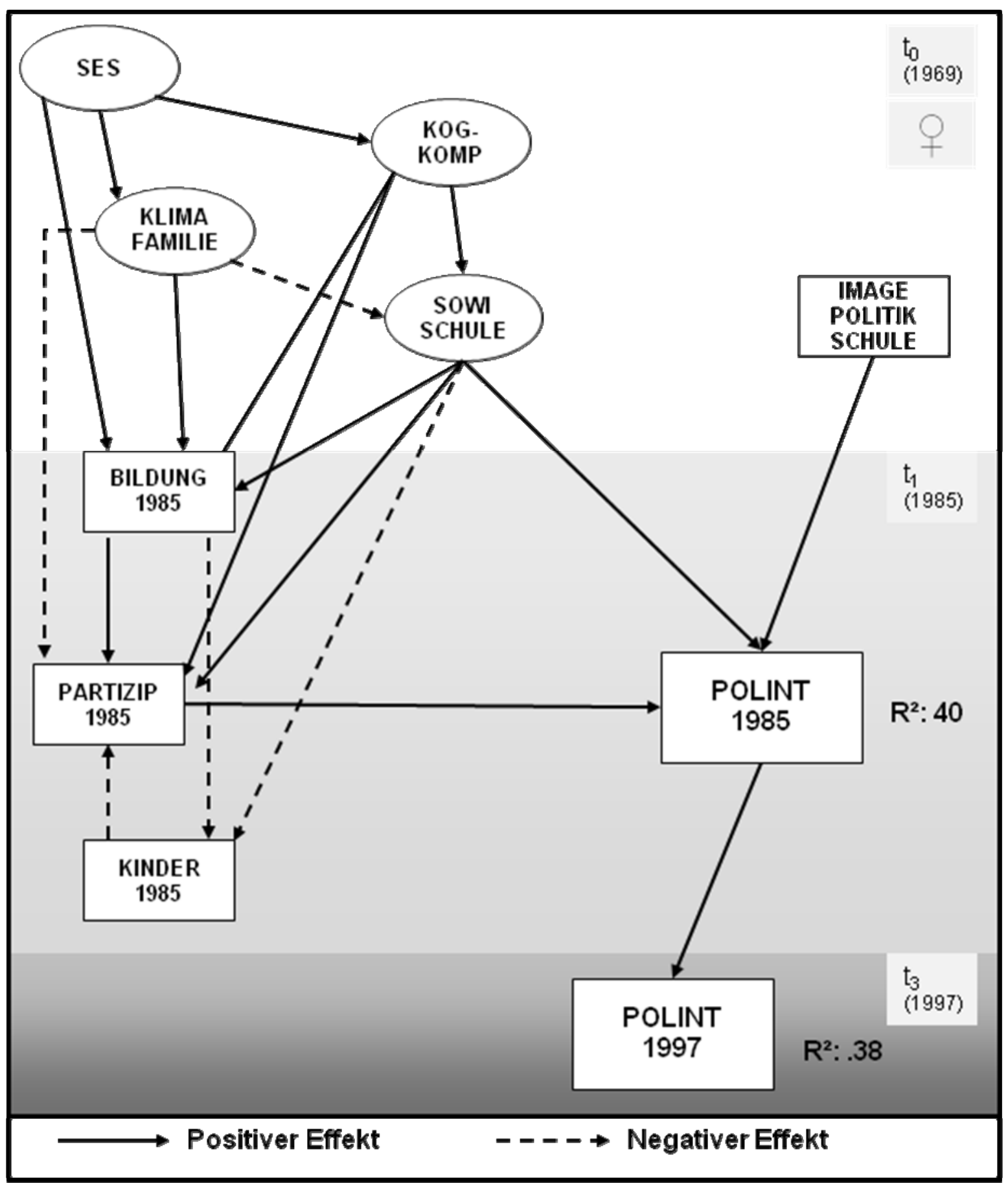


Abbildung 7.3b: Endmodell zur Erklärung des politischen Interesses bei Männern

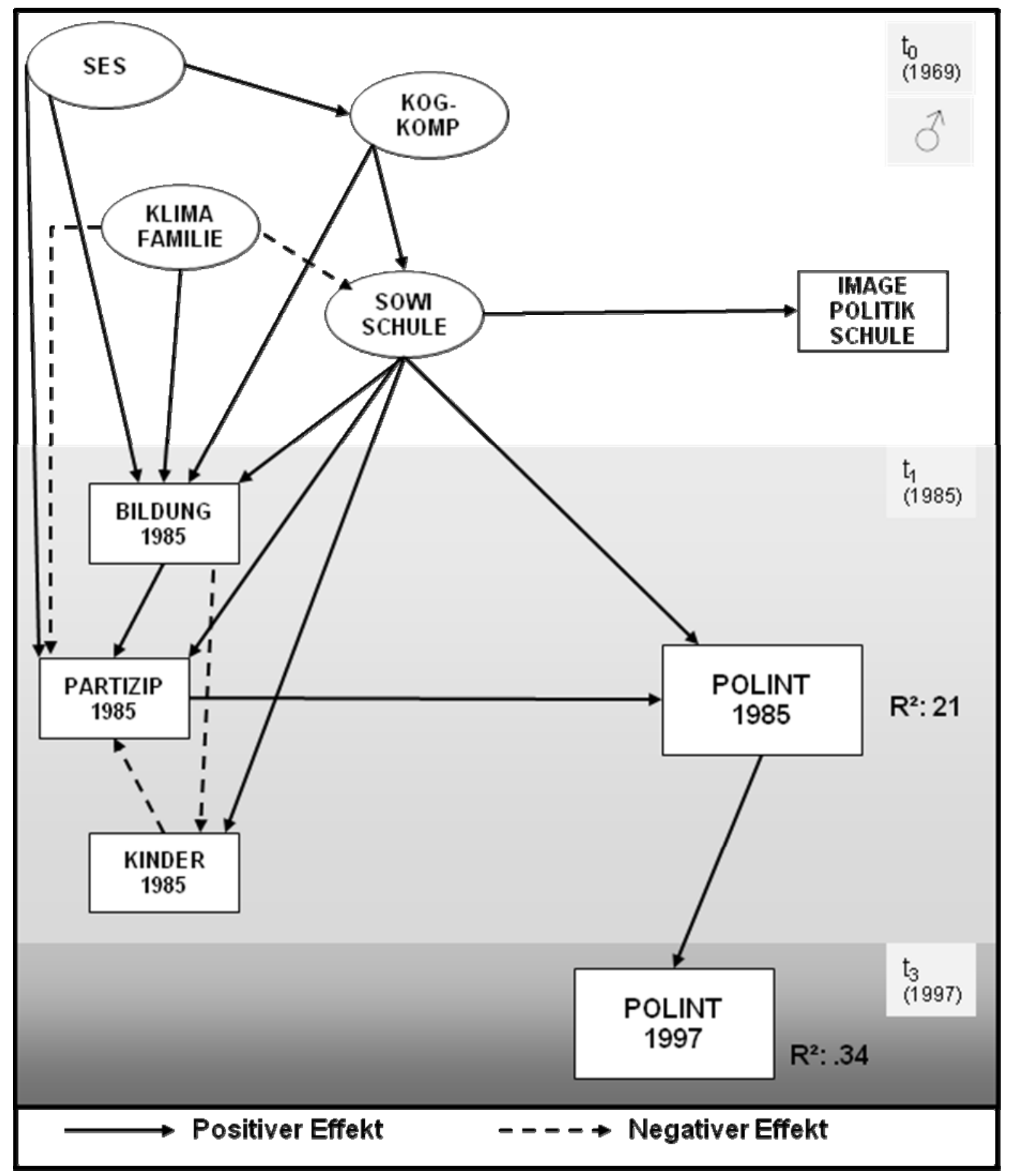

Das Endmodell des multiplen Gruppenvergleichs weist im Vergleich zum Nullmodell bei den Betakoeffizienten einige signifikante Unterschiede zwischen den Geschlechtern auf. Diese beziehen sich im wesentlichen auf die Zusammenhänge zwischen den erklärenden Faktoren untereinander (Beschreibung weiter unten), in einem Punkt aber auch direkt auf die 
abhängige Variable Politikinteresse: Je stärker die weiblichen Jugendlichen die politische Partizipation in der Bewertung der Schulklasse als wichtig wahrnehmen, desto größer ist das politische Interesse der Frauen im späteren Erwachsenenalter.

Betrachtet man zusätzlich die totalen und indirekten Effekte (Tabelle 7.15), so werden weitere Unterschiede zwischen den Geschlechtern sichtbar: Während für Männer die soziale Herkunft eine schwache, aber signifikante Wirkung auf das Politikinteresse ausübt, gilt dieser Zusammenhang für Frauen nicht. Für Frauen dagegen besitzt die kognitive Kompetenz in der Jugend - unabhängig vom später erreichten Bildungsniveau - einen etwas stärkeren Einfluss auf das Politikinteresse im 30. Lebensjahr. Einen gleich starken negativen Effekt hat das Familienklima. Dieser negative Koeffizient ist besonders bemerkenswert, da es der ursprünglichen Hypothese, wonach ein positives Familienklima das Politikinteresse fördern sollte, widerspricht: Ein negatives Familienklima wirkt bei den Heranwachsenden politisierend.

Tabelle 7.15 Gruppenvergleich Geschlecht: totale und indirekte unstandardisierte Effekte

\begin{tabular}{l|c|c}
\hline \multicolumn{1}{c}{ Totale Effekte* } \\
\hline Konstrukt & $\begin{array}{c}\text { Polint85 } \\
\text { Männer }\end{array}$ & $\begin{array}{c}\text { Polint85 } \\
\text { Frauen }\end{array}$ \\
\hline SES & $0.06(0.10)$ & $0.14(0.08)$ \\
\hline KOGKOMP & $0.07(0.05)$ & $-0.13(-0.14)$ \\
\hline KLIMA FAMILIE & $-0.11(-0.14)$ & $0.49(1.05)$ \\
\hline SOWI SCHULE & $0.36(0.98)$ & $0.16(0.18)$ \\
\hline IMAGE POLITIK SCHULE & $0.05(0.02)$ & $0.10(0.02)$ \\
\hline BILDUNG 1985 & $0.27(0.32)$ & $0.36(0.32)$ \\
\hline PARTIZIP 1985 & - & - \\
\hline KINDER 1985 & Polint85 \\
\hline & Polint85 & Frauen \\
\hline Konstrukt & Männer & $0.14(0.08)$ \\
\hline SES & $0.06(0.10)$ & $-0.13(-0.14)$ \\
\hline KOGKOMP & $0.07(0.05)$ & $0.10(0.32)$ \\
\hline KLIMA FAMILIE & $-0.11(-0.14)$ & $0.10(0.02)$ \\
\hline SOWI SCHULE & $0.05(0.15)$ & - \\
\hline IMAGESCHULE & &
\end{tabular}


KINDER 1985

* Aufgeführt sind nur Koeffizienten, die auf dem 5\%-Niveau signifikant sind Zusammengenommen zeigt sich, dass die frühen Sozialisationseinflüsse im Kausalmodell bei Frauen stärker wirksam sind als bei Männern. Die Sozialisationserfahrungen, die nach der mittleren Adoleszenz gesammelt werden (erworbener Bildungsabschluss und Partizipationserfahrungen), weisen bei beiden Geschlechtern die gleiche Effektstärke auf.

Deutlichen Diskrepanzen zwischen Männern und Frauen bestehen auch in der jeweiligen Erklärungskraft der Kausalmodelle: Während für Frauen ein erklärter Varianzanteil von $40 \%$ für das Politikinteresse im 30. Lebensjahr erreicht wird, liegt der Anteil der gebundenen Variation für Männer bei gerade einmal 21\% (Tabelle 7.14).

Darüber hinaus lässt das Kausalmodell - jenseits der abhängigen Variable weitere auffällige Sozialisationsunterschiede zwischen den weiblichen und männlichen ehemaligen Gymnasiasten erkennen (Tabelle 7.14). So zeigt sich beispielsweise, dass für weibliche Befragte in der untersuchten Kohorte die soziale Herkunft sehr viel wichtiger für die Höhe der kognitive Kompetenz und des Bildungserfolgs ist als für Männer. Auch die Auseinandersetzung mit Sozialwissenschaften im Schulkontext hat bei Frauen einen größeren Einfluss auf die spätere Höhe des Bildungsniveaus als bei Männern. Die frühe Beschäftigung mit Sozialwissenschaften in der Schule zeigt zudem einen weiteren bemerkenswerten Effekt: je mehr männliche Jugendliche mit Sozialwissenschaften in der Schule in Kontakt geraten, desto häufiger übernehmen sie in den folgenden fünfzehn Jahren die Betreuung von Kleinkindern. Bei Frauen liegt ein gegenteiliger Effekt vor, sie versorgen umso seltener Kleinkinder, je mehr sie sich in der Schule mit Sozialwissenschaften beschäftigt haben. Man könnte also hier argumentieren, dass sich ,Sozialwissenschaften in der Schule' emanzipierend auf beide Geschlechter auswirkt und Geschlechtsrollenstereotypen reduziert.

\subsubsection{Interaktionseffekte hinsichtlich der Stabilität des Politikinteresses}


Im folgenden Abschnitt wird überprüft, ob, wie in den Hypothesen formuliert, Interaktionseffekte hinsichtlich der Stabilität des Politikinteresses vorliegen (Hypothesen AI1 und AI2). Eine Möglichkeit zur Feststellung von Interaktionseffekten besteht in der simultanen Testung des Kausalmodells in verschiedenen Subgruppen ${ }^{12}$. Mit diesem Verfahren kann überprüft werden, ob die Stabilität des Politikinteresses bei wenig Politikinteressierten und weniger gebildeten Menschen geringer ausgeprägt ist als bei politisch stark Interessierten und höher gebildeten Personen.

Somit werden zwei weitere multiple Gruppenvergleiche berechnet: für die dichotomisierten Variablen Politikinteresse und Bildung ${ }^{13}$.

Die Modellüberprüfung erfolgt auch hier wieder schrittweise: Das jeweilige Ausgangsmodell überprüft, ob sich die Kovarianzmatrizen der beiden Stichproben signifikant voneinander unterscheiden. Wird die Nullhypothese, nach der zwischen den jeweiligen Gruppen keine Unterschiede bestehen, verworfen, so wird untersucht, ob sich das Modell verbessert, wenn der Stabilitätskoeffizient (gerichteter Pfadkoeffizient zwischen Politikinteresse 1985 und Politikinteresse 1997) zwischen den beiden Gruppen zur Schätzung freigegeben wird.

Verbessert sich die Modellanpassung im Vergleich zum Ausgangsmodell signifikant, so kann auch die zweite Nullhypothese, wonach das Politikinteresse in den verglichenen Subgruppen gleich stabil ist, abgelehnt werden.

\footnotetext{
12 Eine weitere Möglichkeit Interaktionseffekte zu untersuchen besteht in der Modellierung eines Interaktionseffektes mit Produktvariablen. Die praktische Umsetzung geschieht in Lisrel mit der Spezifikation nicht-linearer Restriktionen, die über den CO-Befehl formuliert werden können, (siehe auch Reinecke 1999).

13 Für die dichotome Variable, ,Politikinteresse' wurden diejenigen zum Wert 0 zusammengefasst, die 1985 ein sehr schwaches bis mittleres Politikinteresse angaben. Alle mittel bis sehr stark an Politik interessierten erhalten den Wert 1. Die dichotomen Bildungsvariable wurde so kodiert, dass alle, die Mittlere Reife oder Abitur aufweisen den Wert 0 erhalten. Alle diejenigen, die mindestens ein Studium begonnen haben, bekommen den Wert 1 zugewiesen.
} 
Die Tabelle 7.16 zeigt die Modellanpassung für beide Modelle. Die aufgeführten Anpassungswerte machen deutlich, dass durch die freigegebene Schätzung des Stabilitätskoeffizienten eine deutliche Modellverbesserung erreicht werden konnte. Inhaltlich bedeutet dies, dass die nur wenig Politikinteressierten ein signifikant instabileres Interesse an politischen Angelegenheiten aufbringen als stark politikinteressierte Befragte (unstandardisierter Pfadkoeffizient bei wenig Politikinteresse: 0.16 ; bei starkem Politikinteresse: 2.15 )

Tabelle 7.16 Gruppenvergleich zwischen Befragten mit schwachem und starkem Politikinteresse, Modellanpassung für das Ausgangs- und Endmodell

\begin{tabular}{l|l|l|l|l|l}
\hline Modell & $\begin{array}{l}\text { Chi- } \\
\text { Quadrat }\end{array}$ & Df & p & RMSEA & p-Close \\
\hline Ausgangsmodell & 1417.38 & 257 & 0.000 & 0.089 & 0.00 \\
\hline $\begin{array}{l}\text { Modell bei freigesetztem } \\
\text { Pfad zwischen }\end{array}$ & 942.17 & 256 & 0.030 & 0.068 & 0.00 \\
Polint85 und Polint97 & & & & & \\
\hline
\end{tabular}

Die zweite Interaktionshypothese bezieht sich auf das erworbene Bildungsniveau und geht davon aus, dass die 1985 höher gebildeten Personen ein konstanteres Politikinteresse besitzen.

Auch hier ergibt sich ein signifikanter Interaktionseffekt: Nach der Aufhebung der Gleichheitsrestriktion für die Schätzung der Stabilitätskoeffizienten ist eine schwache, aber auf dem 5\%-Niveau signifikante Modellverbesserung zu verzeichnen (Tabelle 7.17). Wie vermutet, verfügen die höhergebildeten Befragten also über ein stabileres Politikinteresse (unstand. Pfadkoeffizient: 0.63), als diejenigen, die ihre Schullaufbahn ,nur' mit Mittlerer Reife oder dem Abitur verlassen haben (unstand. Pfadkoeffizient: 0.48).

Tabelle 7.17 Gruppenvergleich zwischen Befragten mit hoher Bildung und niedrigeren Bildung, Modellanpassung für das Ausgangs- und Endmodell

\begin{tabular}{l|l|l|l|l|l}
\hline Modell & $\begin{array}{l}\text { Chi- } \\
\text { Quadrat }\end{array}$ & Df & p & RMSEA & p-Close \\
\hline Ausgangsmodell & 1177.07 & 257 & 0.000 & 0.079 & 0.00 \\
\hline
\end{tabular}




\begin{tabular}{l|l|l|l|l|l} 
Modell bei freigesetztem & 1153.18 & 256 & 0.000 & 0.078 & 0.00 \\
Pfad zwischen \\
Polint85 und Polint97
\end{tabular}

Zusammenfassend kann gesagt werden, dass die empirischen Ergebnisse ein differenziertes Bild ergeben: Einerseits konnten einige der aufgestellten Hypothesen - insbesondere zur manifesten politischen Sozialisation in der Jugend - bestätigt werden. Darüber hinaus zeigen sich einige bemerkenswerte Unterschiede zwischen den Geschlechtern. Die Interaktionshypothesen im Hinblick auf die Stabilität des Politikinteresses konnten beide bestätigt werden. 


\subsubsection{Spezifikation des Analysemodells für die Wertorientierungen}

In diesem Abschnitt erfolgt zunächst die Überprüfung des nach den Hypothesen BF1 bis BS5 abgeleiteten Kausalmodells für Wertorientierungen. Anschließend wird die Überprüfung der in den Hypothesen BI1 und BI2 formulierten Interaktionseffekte dargestellt.

In einer ersten bivariaten Analyse werden die einfachen Zusammenhänge zwischen den erklärenden Variablen und der abhängigen Variable ,InglehartIndex' untersucht. Dazu werden polychorische Korrelationskoeffizienten berechnet (s. Tabelle 7.19).

Tabelle 7.19 Polychorische Korrelationen der Indikatoren mit postmaterialistischen Wertorientierungen, I ngelhartIndex basierend auf der Untersuchungsstichprobe von max. 1596

\begin{tabular}{l|l}
\hline \multicolumn{1}{c|}{ I ndikatoren } & $\begin{array}{c}\text { I } \\
\text { lehart-I ndex } \\
\mathbf{1 9 8 5}\end{array}$ \\
\hline Treiman-Prestigeskala Vater & 0.029 \\
\hline Schulbildung Vater & 0.053 \\
\hline Schulbildung Mutter & 0.061 \\
\hline Analogie-Test nach Amthauer & $0.128^{*}$ \\
\hline Wortauswahl-Test nach Amthauer & 0.066 \\
\hline Geschlecht & -0.028 \\
\hline Kritik & 0.110 \\
\hline Probleme & $0.151^{*}$ \\
\hline Protesterfahrung in der Schule & 0.103 \\
\hline Beliebteste vs. unbeliebteste Fächer aus dem & 0.092 \\
sozialwissenschaftlichen Bereich & \\
\hline Durchschnittsnote in sozialwissenschaftlichen Fächern & 0.146 \\
\hline Begabung in sozialwissenschaftlichen Fächern & $0.185^{*}$ \\
\hline Politisierung der Schulklasse & 0.010 \\
\hline Gewünschtes Partizipationsalter & -0.146 \\
\hline Partizipationserfahrung bis 1969 (Teilnahme & 0.005 \\
Demonstration) & \\
\hline Mitglied in politischer Gruppe & 0.147 \\
\hline Partizipationserfahrung bis 1985 & $0.414^{*}$ \\
\hline Bildung bis 1985 & $0.202^{*}$ \\
\hline Betreuung Kleinkinder bis 1985 & -0.054 \\
\hline Berufserfahrung bis 1985 & $-0.179 *$ \\
\hline Berufsprestige 1985 & 0.059 \\
\hline &
\end{tabular}


Die bivariate Korrelationsanalyse zeigt bei allen Koeffizienten (abgesehen vom Geschlecht, hier wurde keine explizite Hypothese formuliert) hypothesenkonforme Zusammenhänge. Diese sind allerdings, wie auch beim Politikinteresse, nicht immer substanziell und signifikant.

Wie bei der Analyse zum politischen Interesse werden daher - abgesehen von den Hintergrundvariablen (Geschlecht, Kognitive Kompetenz, SES) und der latenten Sozialisationsvariable ,PROTEST SCHULE' - in die Pfadanalyse nur solche Variablen bzw. Konstrukte einbezogen, die auf der bivariaten Ebene einen Korrelationskoeffizienten von mindestens 0.15 bei einer Irrtumswahrscheinlichkeit von $<5 \%$ aufweisen. Dabei handelt es sich um folgende Variablen (bzw. Konstrukte):

- Geschlecht (GESCHL) ${ }^{64}$

- Sozio-ökonomischer Status in der Jugendzeit (SES)

- Kognitive Kompetenz in der Jugendzeit (KOGKOMP)

- Erziehungsziel Selbständiges Denken und Handeln (ERZSELBST)

- Kontakt mit Sozialwissenschaften im Schulkontext (SOWI SCHULE)

- Protesterfahrungen in der Schule (PROTEST SCHULE)

- Erreichter Bildungsabschluss bis 1985 (BILDUNG 1985)

- Partizipationserfahrung bis 1985 (PARTIZIP 1985)

- Dauer der Erwerbstätigkeit bis 1985 (DAUER ERWERB 1985)

Das zur Überprüfung der Hypothesen spezifizierte Ausgangsmodell zur Erklärung der postmaterialistischen Wertorientierungen ist in Abbildung 7.4 wiedergegeben.

${ }^{64}$ In den Klammen sind die Kurzbezeichungen für die Konstrukte aufgeführt. 


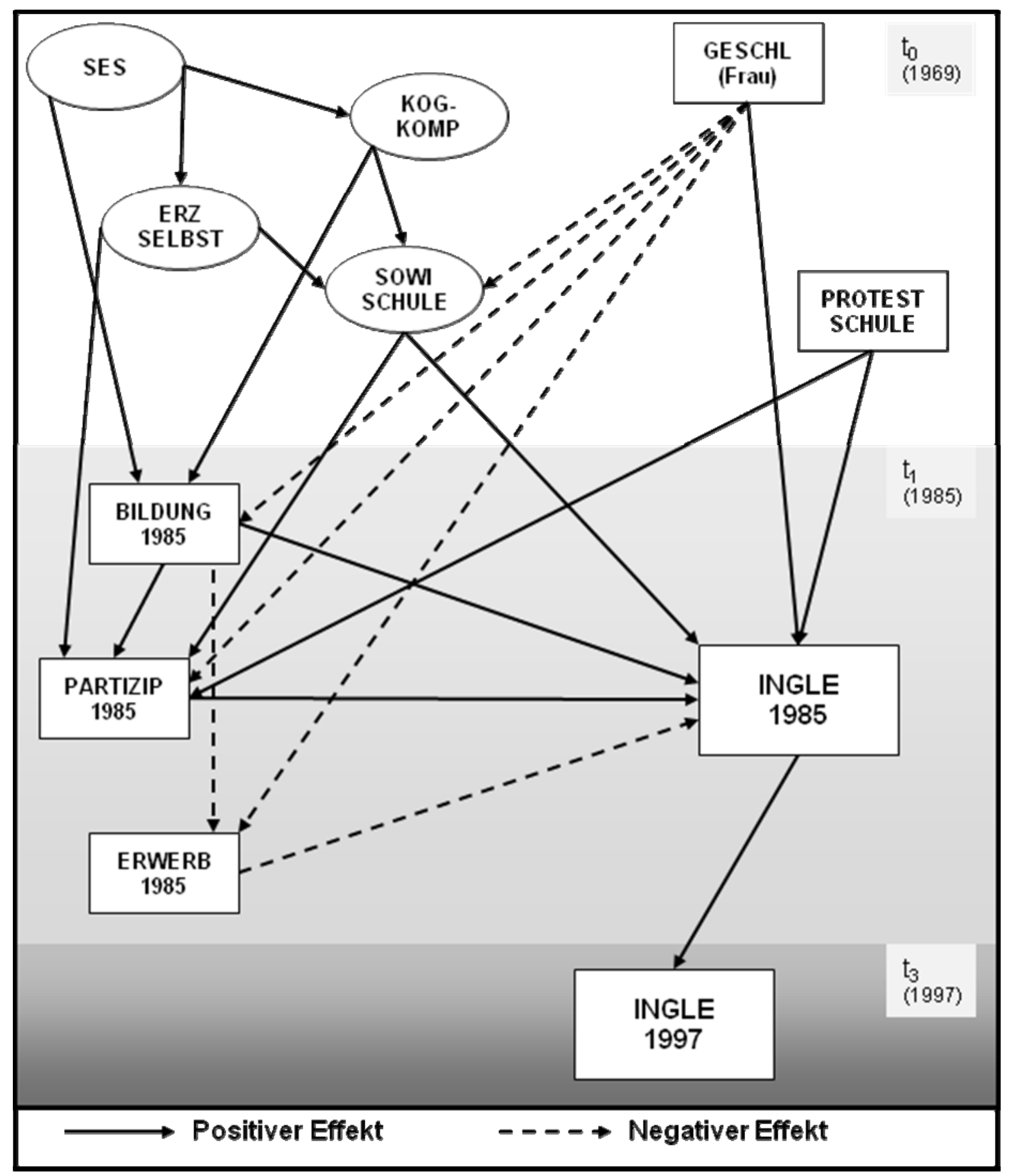


Die Werte für die Modellanpassung des Nullmodells können der Tabelle 7.20 entnommen werden.

Tabelle 7.20: Modellanpassung für das Ausgangsmodell

\begin{tabular}{l|l|l|l|l|l}
\hline $\begin{array}{l}\text { Chi- } \\
\text { Quadrat }\end{array}$ & df & $\mathbf{p}$ & RMSEA & p-Close & $\mathbf{N}$ \\
\hline 318.36 & 105 & 0.000 & 0.042 & 0.99 & 1157 \\
\hline
\end{tabular}

Die in der Tabelle 7.20 aufgeführten Anpassungswerte (RMSEA und p-close) zeigen zwar eine recht gute Modellanpassung, einige der standardisierten Residuen und Modifikationsindizes ${ }^{65}$ deuten allerdings auf eine ungenügende Modellanpassung bzw. Fehlspezifikationen hin. Zur Verbesserung der Anpassung werden auch hier wieder (vgl Kapitel 7.2.2) mit Hilfe der Modifikationsindizes schrittweise Modellmodifikationen durchgeführt, sofern diese inhaltlich interpretierbar sind. Beendet wird die Modellanpassung dann, wenn sich mit weiteren Veränderungen bei den Pfaden zwischen den latenten Variablen keine relevante Modellverbesserung mehr ergibt (Modifikationsindizes $<7$ ).

Die Tabelle 7.21 gibt die Modellanpassung für das Endmodell wieder. Im Vergleich zum Ausgangsmodell hat sich die Modellanpassung auch hier deutlich verbessert (Tabelle 7.21).

\section{Tabelle 7.21: Modellanpassung für das modifizierte Modell, Endmodell}

\begin{tabular}{l|l|l|l|l|l}
\hline $\begin{array}{l}\text { Chi- } \\
\text { Quadrat }\end{array}$ & Df & $\mathbf{p}$ & RMSEA & p-Close & $\mathbf{N}$ \\
\hline 192.96 & 104 & 0.000 & 0.027 & 1 & 1157 \\
\hline
\end{tabular}

Das modifizierte Strukturmodell für die Wertorientierungen ist in der Abbildung 7.5 wiedergegeben. Die entsprechenden Beta-Koeffizienten sind in Tabelle 7.22 abgebildet (nur Koeffizienten, die mindestens auf dem 5\%-

${ }^{65}$ Vgl. 'ingle ausgangsmodell. pdf'. 
Niveau signifikant sind). Die Tabelle 7.23 enthält die totalen und indirekten Effekte.

Abbildung 7.5 Endmodell zur Erklärung der Wertorientierungen

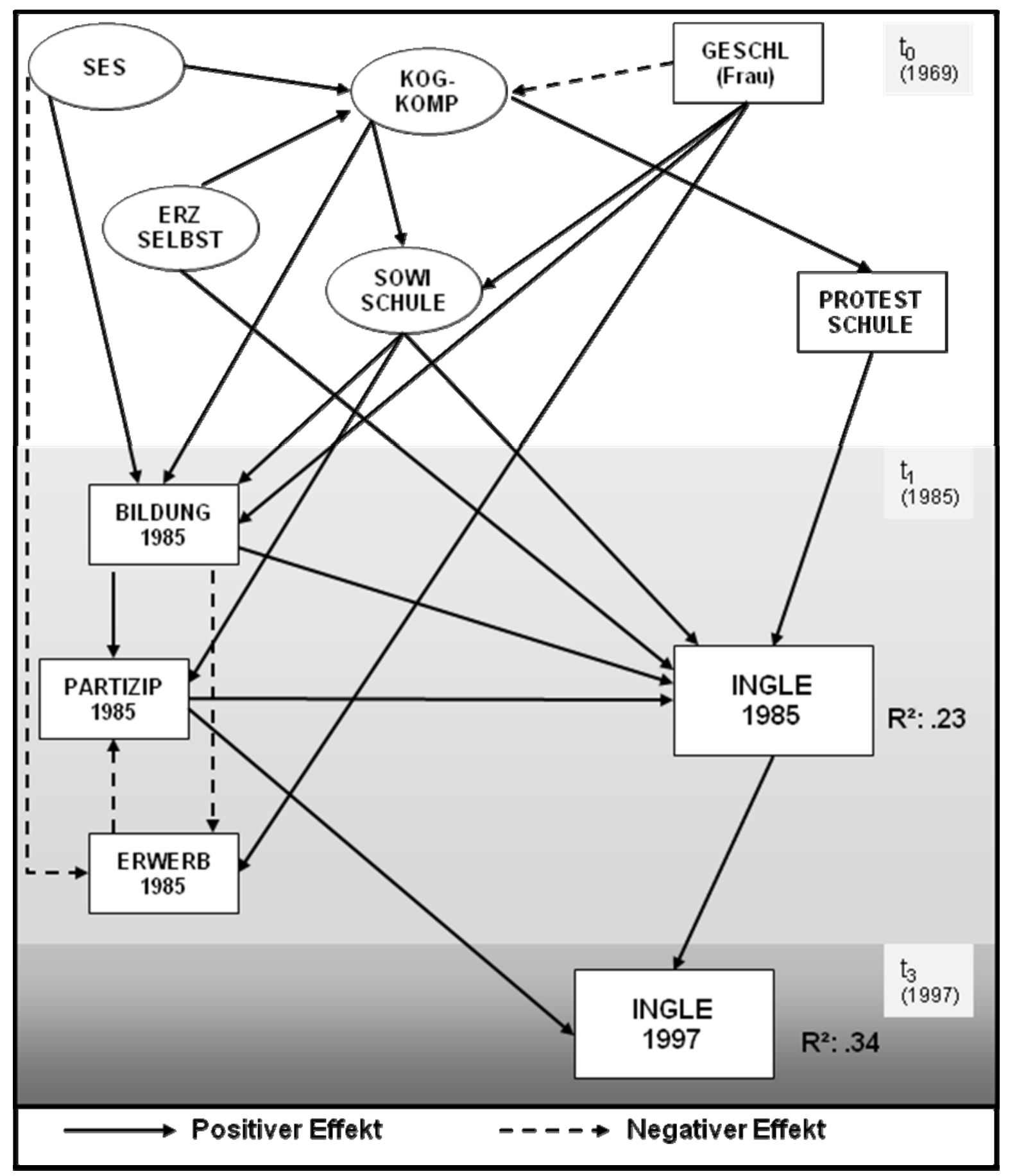




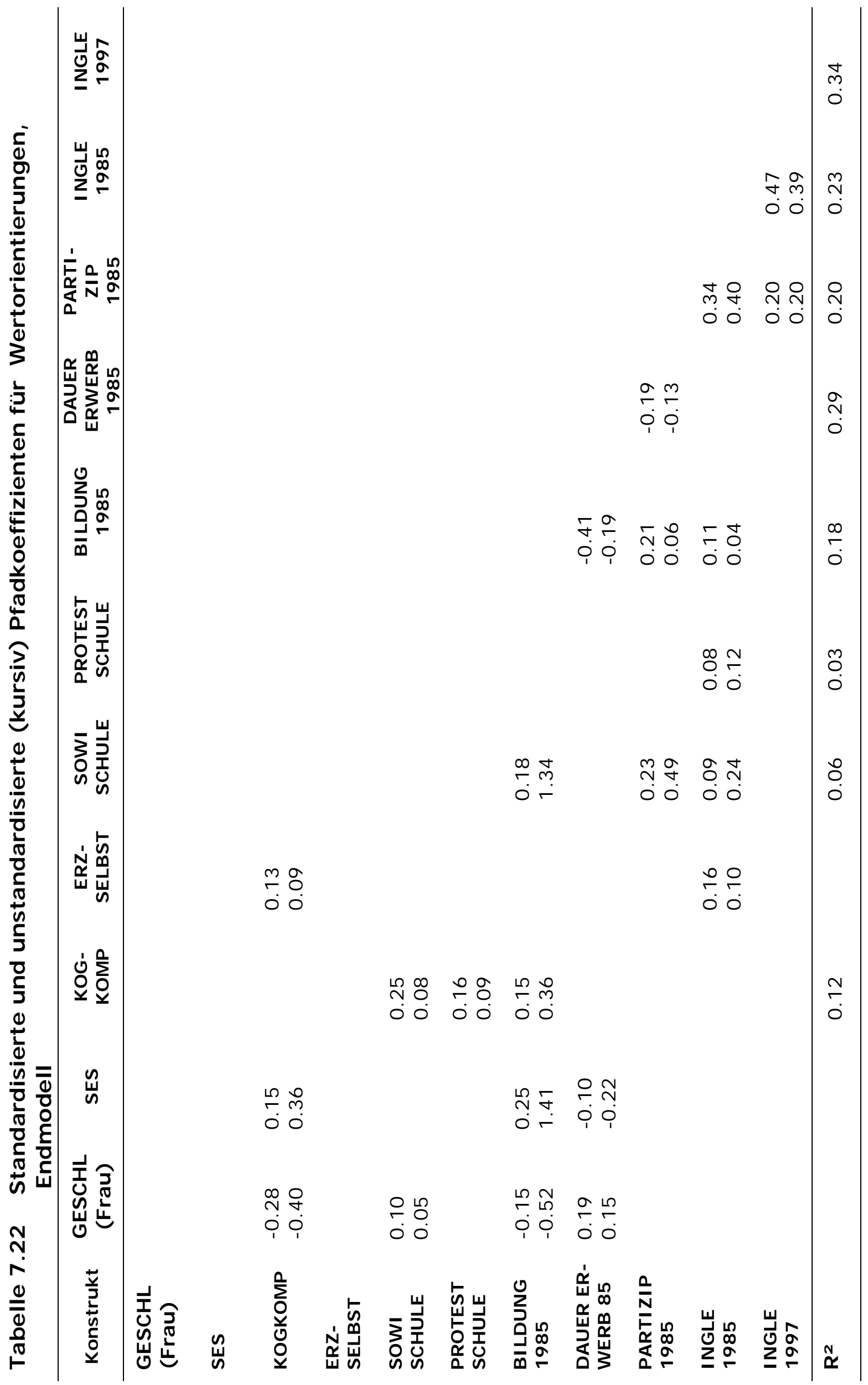


Wie in den Tabellen 7.22 und 7.23 zu sehen ist, spielt der Faktor Geschlecht anders als bei der Erklärung des politischen Interesses - für die Wertorientierungen im Erwachsenenalter fast keine Rolle (unstandardisierter, indirekter Effekt von -0.05). Einen relativ geringen und indirekt verlaufenden Einfluss weisen auch die beiden Faktoren ,Sozio-ökonomische Stellung des Elternhauses' (SES) und ,jugendliche kognitive Kompetenz' (KOGKOMP) auf. Ein eigenständiger und direkter Effekt geht hingegen von der latenten Sozialisation im Elternhaus aus: Je mehr Wert Eltern auf die Erziehung zur Selbstständigkeit (ERZSELBST) legen, desto postmaterialistischer sind die späteren Wertorientierungen ihrer Kinder.

Tabelle 7.23 Totale und indirekte Effekte auf Wertorientierungen 1985, standardisierte Effekte (in Klammern und kursiv unstandardisiert)

Totale Effekte*

\begin{tabular}{|c|c|}
\hline Konstrukt & I NGLE85 \\
\hline GESCHL & $-0.05(-0.05)$ \\
\hline SES & $0.08 \quad(0.15)$ \\
\hline KOGKOMP & $0.10 \quad(0.08)$ \\
\hline ERZSELBST & $0.18 \quad(0.11)$ \\
\hline SOWI SCHULE & $0.21 \quad(0.53)$ \\
\hline PROTEST SCHULE & $0.08 \quad(0.10)$ \\
\hline BILDUNG 1985 & $0.22 \quad(0.07)$ \\
\hline PARTIZIP1985 & $0.34 \quad(0.40)$ \\
\hline DAUER ERWERB 1985 & $-0.06(-0.05)$ \\
\hline
\end{tabular}

Indirekte Effekte*

\begin{tabular}{|c|c|}
\hline Konstrukt & I NGLE85 \\
\hline GESCHL & $-0.05(-0.05)$ \\
\hline SES & $0.08 \quad(0.15)$ \\
\hline KOGKOMP & $0.10 \quad(0.08)$ \\
\hline ERZSELBST & $0.01 \quad(0.01)$ \\
\hline SOWI SCHULE & $0.11 \quad(0.15)$ \\
\hline PROTEST SCHULE & $-(-)$ \\
\hline BILDUNG 1985 & $0.10 \quad(0.04)$ \\
\hline PARTIZIP1985 & $-(-)$ \\
\hline DAUER ERWERB 1985 & $-0.06(-0.05)$ \\
\hline
\end{tabular}

* Alle aufgeführten Koeffizienten sind auf dem $5 \%$-Niveau signifikant 
Ebenfalls signifikant sind die Einflüsse der schulischen Sozialisation: Die Wertorientierungen im Erwachsenalter sind umso postmaterialistischer, je stärker sich die Jugendlichen im Unterricht mit sozialwissenschaftlichen Themen beschäftigt haben und je umfangreicher ihre schulischen Protesterfahrungen sind.

Der stärkste Effekt geht von der politischen Partizipationserfahrung aus. Allerdings gilt auch hier, dass die Kausalrichtung des Einflusses nicht eindeutig ist: Entwickeln sich postmaterialistische Wertorientierungen primär, weil man Erfahrungen mit politischer Partizipation gesammelt hat? Oder ist die politische Partizipation bei Postmaterialisten höher? Oder aber bestehen Wechselwirkungen (s. auch Kapitel 7.2.1)?

Ebenfalls hypothesenkonform verläuft der Einfluss des Bildungsniveaus; d.h., selbst in der stark bildungsprivilegierten Kohorte der ehemaligen Gymnasiasten ist noch ein positiver Bildungseffekt spürbar. Auch der Einfluss, den die Erwerbstätigkeit ausübt steht im Einklang mit den Hypothesen: Die ehemaligen Gymnasiasten sind umso materialistischer, je länger sie - unabhängig vom Bildungsniveau - einer Erwerbstätigkeit nachgegangen sind. 


\subsubsection{Geschlechtsspezifische Unterschiede bei der Erklärung der Wertorientierungen}

Die Hypothese, dass bei der Erklärung Wertorientierungen Geschlechtsunterschiede in der Kausalstruktur vorliegen, wird mit Hilfe des multiplen Gruppenvergleichs untersucht. Die Vorgehensweise bei der Modellanpassung entspricht der im vorangegangenen Kapitel beschriebenen Strategie (Gruppenvergleich für das Politikinteresse).

Die folgende Tabelle gibt die Modellanpassung für das Ausgangs- und Endmodell wieder:

Tabelle 7.24 Gruppenvergleich zwischen Männern und Frauen, Modellanpassung für das Ausgangs- und Endmodell ${ }^{66}$

\begin{tabular}{l|l|l|l|l|l}
\hline Modell & $\begin{array}{l}\text { Chi- } \\
\text { Quadrat }\end{array}$ & Df & p & RMSEA & p-Close \\
\hline Ausgangsmodell & 1510.49 & 230 & 0.000 & 0.098 & 0.000 \\
\hline Endmodell & 379.23 & 207 & 0.000 & 0.038 & 1 \\
\hline
\end{tabular}

In der Tabelle 7.25 sind die Pfadkoeffizienten des Endmodells ausgewiesen. Signifikante Unterschiede zwischen Männern und Frauen werden besonders hervorgehoben (unterstrichen).

\footnotetext{
${ }^{66}$ Wie auch beim Politikinteresse wurden die Faktorladungen im Endmodell gleichgesetzt. Die Messfehler- und Faktorvarianzen sowie die Betakoeffizienten konnten z.T. gleichgesetzt werden, in anderen Fällen wurden sie frei geschätzt. Die Details des Endmodells können der Datei ,inglegrup endmodell.pdf' auf der beiliegenden CD entnommen werden.
} 


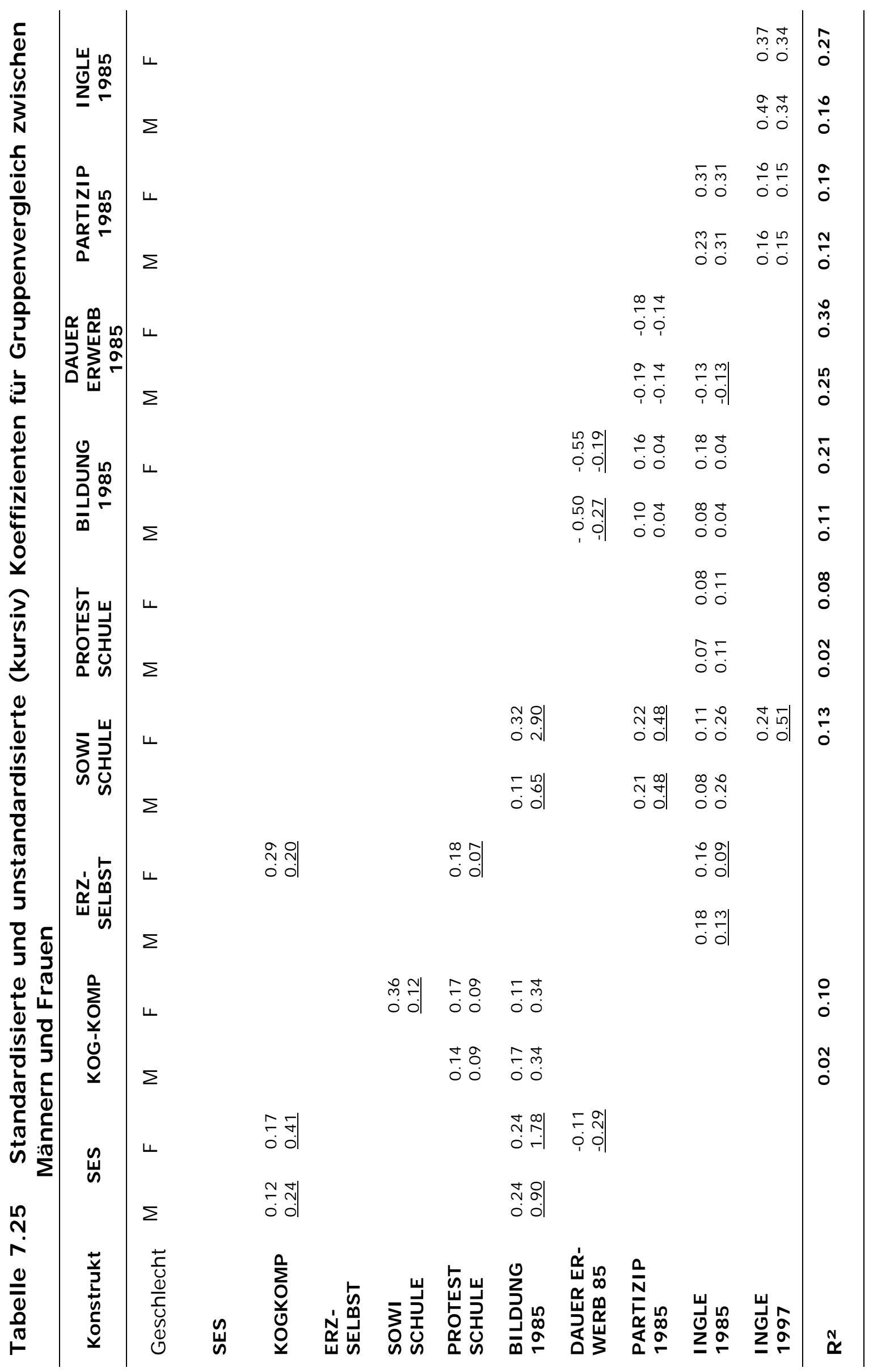


Das Endmodell des multiplen Gruppenvergleichs weist bei den Betakoeffizienten mehrere signifikante Unterschiede zwischen den Geschlechtern auf. Die in Kapitel 7.2.1 beschriebenen Geschlechtsdifferenzen in der Kausalstruktur finden sich z. T. auch hier wieder.

Im Hinblick auf Wertorientierungen unterscheiden sich Männer und Frauen folgendermaßen:

- Bei Frauen entwickeln sich mit höherer kognitiver Kompetenz in der Jugend stärker postmaterialistische Werthaltungen als bei Männern.

- Anders als bei Männern fördert die manifeste Sozialisation in der Schule (SOWI SCHULE) bei Frauen direkt postmaterialistische Wertorientierungen sowohl im 30. Lebensjahr als auch im Alter von 43 Jahren. Interessanterweise ist der Effekt auf das 43. Lebensjahr sogar etwas stärker als derjenige auf die Wertorientierungen im Alter von 30 Jahren. Bei Männern hingegen wirken die schulischen Sozialisationserfahrungen nur auf die Werthaltungen im Alter von 30. Lebensjahr.

- Eine weitere substanzielle Differenz besteht im Einfluss der Erwerbstätigkeit auf die Wertorientierungen. Während bei Männern ein sichtbarer, direkter und indirekter negativer Effekt der Dauer der Erwerbstätigkeit auf postmaterialistische Wertorientierung besteht (unstandardisierter totaler Effekt: -0.16 ), spielt die Erwerbstätigkeit für Frauen eine nur marginale und indirekte Rolle (unstandardisierter totaler Effekt: -0.04). 
Tabelle 7.26 Gruppenvergleich Geschlecht für Wertorientierungen 1985: totale und indirekte standardisierte Effekte (in Klammern und kursiv unstandardisiert)

Totale Effekte*

\begin{tabular}{l|c|c}
\hline Konstrukt & $\begin{array}{c}\text { I NGLE85 } \\
\text { Männer }\end{array}$ & $\begin{array}{c}\text { I NGLE85 } \\
\text { Frauen }\end{array}$ \\
\hline SES & $0.05(0.10)$ & $0.09(0.17)$ \\
\hline KOGKOMP & $0.04(0.04)$ & $0.14(0.10)$ \\
\hline ERZSELBST & $0.18(0.13)$ & $0.21(0.12)$ \\
\hline SOWI SCHULE & $0.15(0.47)$ & $0.26(0.59)$ \\
\hline PROTEST SCHULE & $0.07(0.11)$ & $0.08(0.11)$ \\
\hline BILDUNG 1985 & $0.19(0.10)$ & $0.26(0.06)$ \\
\hline PARTIZIP 1985 & $0.23(0.31)$ & $0.31(0.31)$ \\
\hline DAUER ERWERB 1985 & $-0.17(-0.17)$ & $-0.06(-0.04)$
\end{tabular}

Indirekte Effekte*

\begin{tabular}{l|c|c}
\hline Konstrukt & $\begin{array}{c}\text { I NGLE85 } \\
\text { Männer }\end{array}$ & $\begin{array}{c}\text { I NGLE85 } \\
\text { Frauen }\end{array}$ \\
\hline SES & $0.05(0.10)$ & $0.09(0.17)$ \\
\hline KOGKOMP & $0.04(0.04)$ & $0.14(0.10)$ \\
\hline ERZSELBST & -- & $0.05(0.03)$ \\
\hline SOWI SCHULE & $0.07(0.22)$ & $0.15(0.34)$ \\
\hline PROTEST SCHULE & -- & -- \\
\hline BILDUNG 1985 & $0.11(0.06)$ & $0.08(0.02)$ \\
\hline PARTIZIP 1985 & -- & -- \\
\hline DAUER ERWERB 1985 & $-0.04(-0.04)$ & $-0.06(-0.04)$ \\
\hline
\end{tabular}

* Alle aufgeführten Koeffizienten sind auf dem 5\%-Niveau signifikant

Zwei weitere Unterschiede in der Sozialisation von Männern und Frauen beziehen sich auf Erwerbstätigkeit und latente Sozialisation in der Schule: Frauen, deren Eltern höheren sozialen Schichten angehören, verfügen unabhängig vom Bildungsniveau - über weniger Berufserfahrung. Für Männer spielt dagegen die soziale Herkunft in diesem Zusammenhang keine Rolle.

Bei weiblichen Gymnasiasten führt darüber hinaus die Erziehung zur Selbstständigkeit im Elternhaus zu höherer kognitiven Kompetenz und zu häufigeren Protesterfahrungen in der Schule.

Die jeweiligen modifizierten Strukturmodelle für beide Geschlechter sind in den Abbildungen 7.6a und 7.6b wiedergegeben. 
Abbildung 7.6a Endmodell zur Erklärung der Wertorientierungen bei Frauen

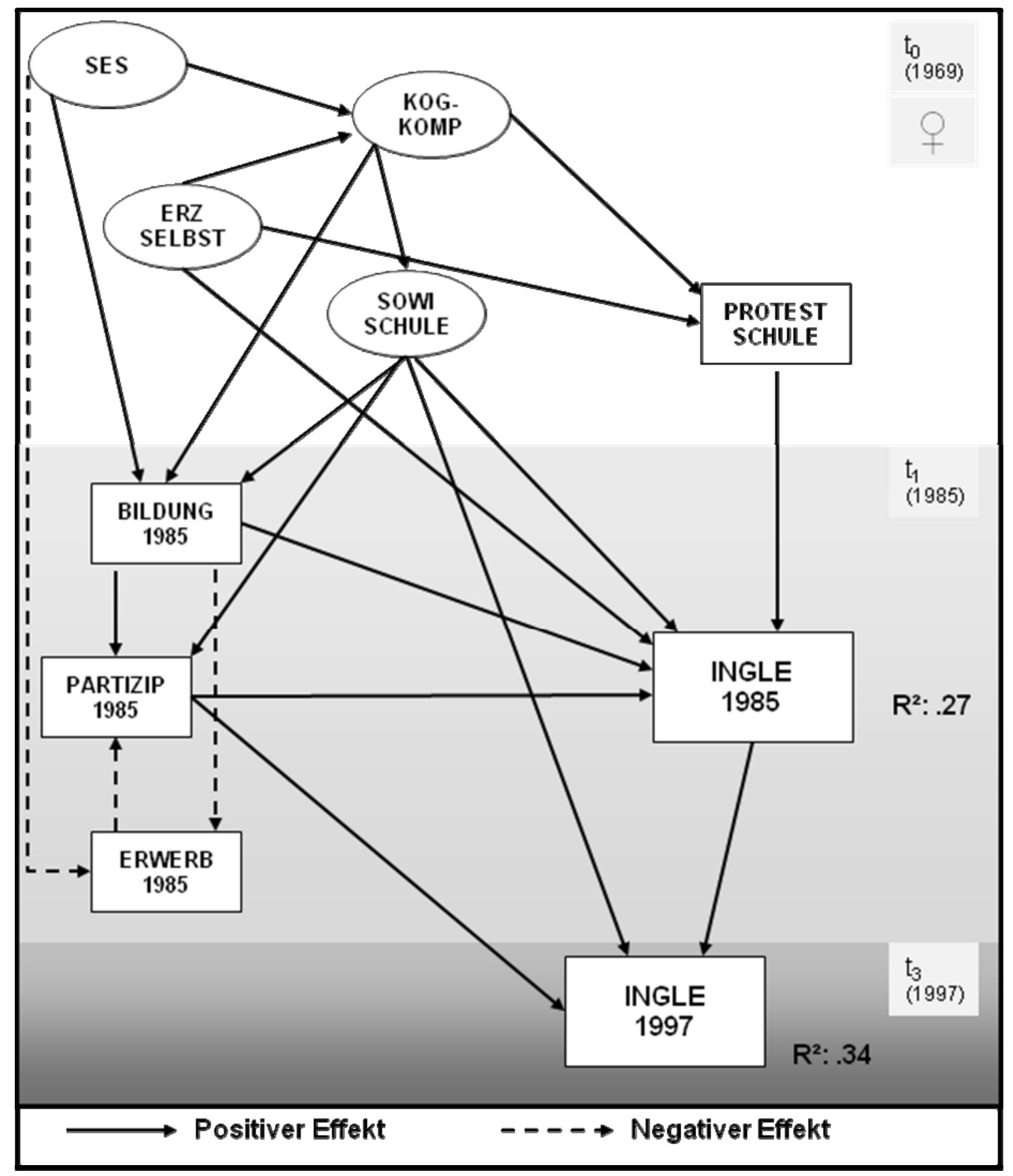




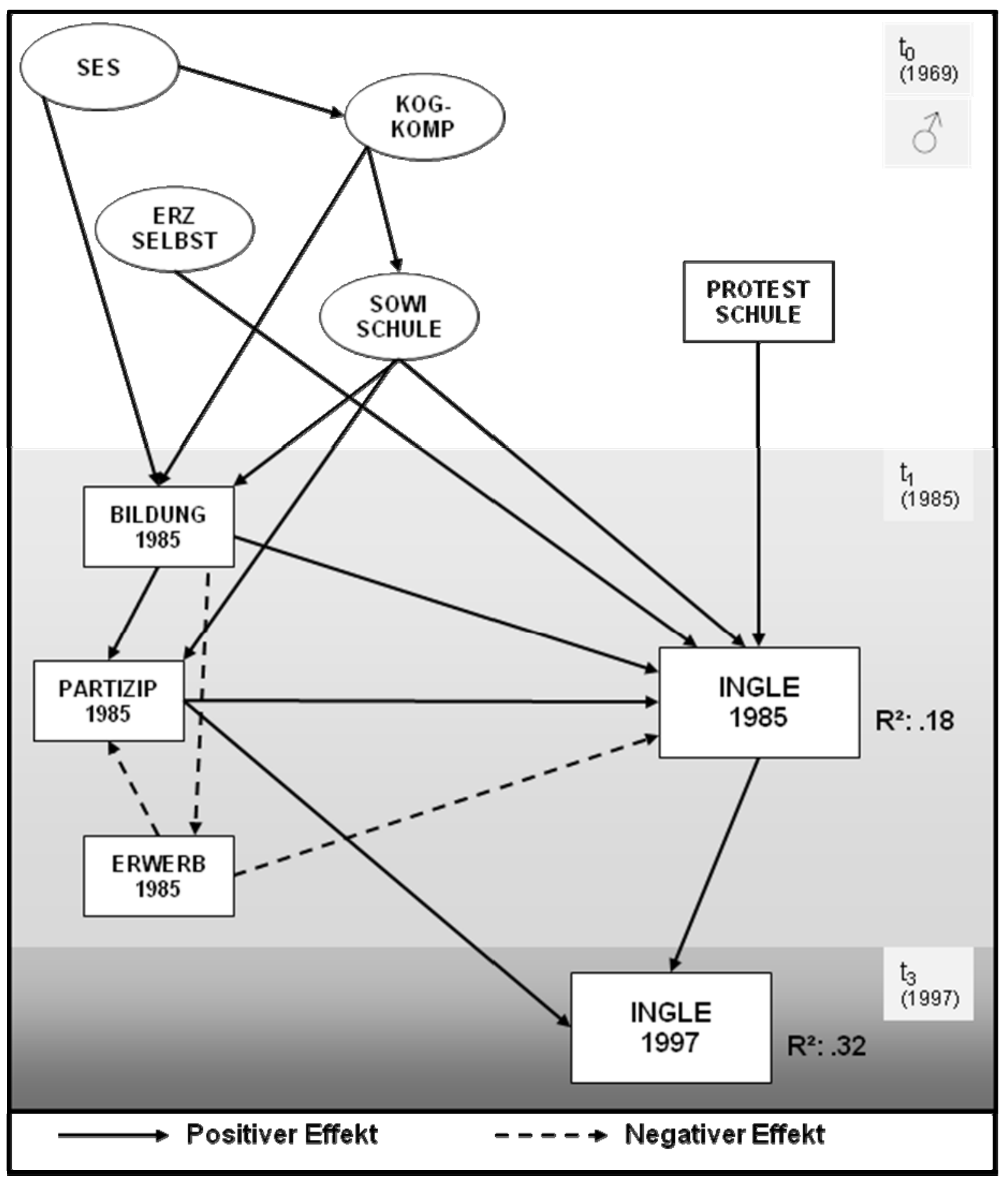

Vergleicht man die Endmodelle für Männer und Frauen (Abbildungen 7.6a und 7.6b) miteinander, so fällt außerdem auf, dass für Frauen zwischen den einzelnen Faktoren deutlich häufiger signifikante (und meist auch stärkere) Beziehungen bestehen. Dies führt zu einem letzten Unterschied zwischen den Geschlechtern: Wie beim Politikinteresse auch, lassen sich die Unterschiede 
in den Werthaltungen der Frauen durch die spezifizierten Kausalmodelle deutlich besser erklären als jene der Männer (Tabelle 7.26). Der Anteil der erklärten Varianz bei Frauen im 30. Lebensjahr liegt bei 27\%, der für Männer nur bei 16\% (vgl. hierzu auch Kapitel 8).

Ein abschließender Überblick über die im Kausalmodell überprüften Hypothesen und deren Bestätigung findet sich in Tabelle 7.27:

Tabelle 7.27 Übersicht über die im Kausalmodell getesteten Hypothesen zu Wertorientierungen

\begin{tabular}{|c|c|c|c|c|c|}
\hline \multirow[t]{3}{*}{ Hypothese } & \multirow[t]{3}{*}{ Kurzbeschreibung } & \multicolumn{4}{|c|}{ Effekt* } \\
\hline & & \multicolumn{2}{|c|}{ direkt } & \multicolumn{2}{|c|}{ indirekt } \\
\hline & & $\mathbf{M}$ & $\mathbf{F}$ & $\mathbf{M}$ & $\mathbf{F}$ \\
\hline BF1 & SES -> ,Wertorientierungen“ & & & $x$ & $\mathrm{x}$ \\
\hline BF2 & KOGKOMP -> ,Wertorientierungen“ & & & $x$ & $x$ \\
\hline BF4 & ERZSELBST -> ,Wertorientierungen“ & $x$ & $x$ & & $x$ \\
\hline BF5 & PROTEST SCHULE -> ,Wertorientierungen“ & $x$ & $\mathrm{x}$ & & \\
\hline BF6 & SOWI SCHULE -> ,Wertorientierungen“ & $x$ & $x$ & $x$ & $x$ \\
\hline BS1 & BILDUNG -> ,Wertorientierungen“ & $x$ & $x$ & $x$ & $x$ \\
\hline BS4 & ERWERB -> ,Wertorientierungen“ & $x$ & & $x$ & $\mathrm{x}$ \\
\hline BS5 & PARTIZIP -> ,Wertorientierungen“ & $x$ & $x$ & & \\
\hline \multicolumn{6}{|c|}{ x Hypothese bestätigt $\quad-$ Hypothese nicht bestätigt } \\
\hline \multicolumn{6}{|c|}{ * nur auf 5\%-Niveau signifikante Effekte angegeben } \\
\hline \multicolumn{6}{|c|}{ **negativer Effekt entgegen den Erwartungen } \\
\hline \multicolumn{6}{|l|}{ M=Männer } \\
\hline \multicolumn{6}{|l|}{$\mathrm{F}=$ Frauen } \\
\hline
\end{tabular}




\subsubsection{Interaktionseffekte hinsichtlich der Stabilität der Wertorientierungen}

Im folgenden Abschnitt wird - wieder mit Hilfe der multiplen Gruppenvergleiche - überprüft, ob auch hinsichtlich der Stabilität von Wertorientierungen Interaktionseffekte vorliegen (Hypothesen $\mathrm{BI} 1$ und $\mathrm{BI}$ ). Das Verfahren entspricht der im Kapitel 7.2.1.2 beschriebenen Vorgehensweise.

Der erste multiple Gruppenvergleich überprüft, ob wenig politikinteressierte Befragte über instabilere Wertorientierungen verfügen als solche Personen, die stark an politischen Angelegenheiten interessiert sind.

Die Tabelle 7.27 zeigt die Modellanpassung für das Ausgangsmodell (Stabilität gleichgesetzt) sowie die Anpassungswerte für das Modell, in dem der Pfad zwischen ,INGLE85' und ,INLGE97' zur Schätzung freigegeben ist. Der Chi-Quadrat-Differenz-Test ergibt für die Modellmodifikation eine leichte Modellverbesserung. Allerdings zeigt sich bei Inspektion der unstandardisierten Koeffizienten, dass entgegen der Hypothese nicht Befragte mit hohem, sondern solche mit niedrigem Politikinteresse über stabilere Werthaltungen verfügen (beta $=0.33$ für hohes und beta $=0.56$ für niedriges Politikinteresse). Die ursprüngliche Hypothese muss somit zurückgewiesen werden.

Tabelle 7.28 Gruppenvergleich zwischen Befragten mit schwachem und starkem Politikinteresse, Modellanpassung für das Ausgangs- und Endmodell

\begin{tabular}{l|l|l|l|l|l}
\hline Modell & $\begin{array}{l}\text { Chi- } \\
\text { Quadrat }\end{array}$ & Df & p & RMSEA & p-Close \\
\hline Ausgangsmodell & 2941.28 & 257 & 0.000 & 0.135 & 0.000 \\
\hline $\begin{array}{l}\text { Modell bei freigesetztem } \\
\text { Pfad zwischen } \\
\text { INGLE85 und INGLE97 }\end{array}$ & 2894.07 & 256 & 0.000 & 0.134 & 0.000 \\
\hline
\end{tabular}


Die zweite Interaktionshypothese behauptet, dass höher gebildete Personen konstantere Wertorientierungen besitzen als solche mit geringerem Bildungsniveau.

Tabelle 7.29 Gruppenvergleich zwischen Befragten mit hoher Bildung und niedrigeren Bildung, Modellanpassung für das Ausgangs- und Endmodell

\begin{tabular}{l|l|l|l|l|l}
\hline Modell & $\begin{array}{l}\text { Chi- } \\
\text { Quadrat }\end{array}$ & Df & p & RMSEA & p-Close \\
\hline Ausgangsmodell & 2255.34 & 257 & 0.000 & 0.116 & 0.000 \\
\hline $\begin{array}{l}\text { Modell bei freigesetztem } \\
\text { Pfad zwischen } \\
\text { INGLE85 und INGLE97 }\end{array}$ & 2252.41 & 256 & 0.000 & 0.116 & 0.000 \\
\hline
\end{tabular}

Auch hier bestätigt sich die in Kapitel 7.2.1.2 formulierte Hypothese nicht. Nach der Aufhebung der Gleichheitsrestriktion für die Schätzung der Stabilitätskoeffizienten zeigt sich keine signifikante Verbesserung der Modellanpassung: höhergebildete Befragte verfügen nicht über stabileren Werthaltungen als die weniger gebildeten ehemaligen Gymnasiasten 


\section{$8 \quad$ Zusammenfassung und Diskussion}

Ausgangspunkt dieser Arbeit ist die oft formulierte, aber nur selten geprüfte Hypothese, die mittlere Jugendzeit sei eine besonders wichtige und ,formative Phase' für die Ausbildung der individuellen politischen Identität.

Im Zentrum der empirischen Untersuchung steht daher die Frage nach dem Einfluss politischer Sozialisationserfahrungen aus der Zeit der mittleren Adoleszenz auf verschiedene Aspekte der politischen Identität im Erwachsenenalter.

Als Datenbasis wurde eine dreiwellige, über 27 Jahre laufende Panelstudie ehemaliger Gymnasiasten verwendet, die das Kölner Institut für Angewandte Sozialforschung im Jahr 1969 initiierte. Mit Hilfe dieses Datensatzes war es möglich, die Bedeutung politischer Sozialisationserfahrungen in der Jugendzeit wie der späteren Lebensphase auf Politikinteresse und Wertorientierungen zu untersuchen.

Da die Untersuchung früher Sozialisationseffekte nur dann sinnvoll ist, wenn die Zielvariablen ein Mindestmaß an Stabilität aufweisen, wurde zunächst die Stabilität von politischem Interesse und politischen Wertorientierungen im Erwachsenenalter untersucht.

Es konnte gezeigt werden, dass beide Zielvariablen ein hohes Maß an Stabilität aufweisen ${ }^{37}$. Die Stabilität des Politikinteresses erwies sich dabei als etwas höher als die der Wertorientierungen ${ }^{38}$.

\footnotetext{
37 Die Höhe der Stabilität ist umso bemerkenswerter, wenn man bedenkt, dass zwischen beiden Messzeitpunkten ein Zeitraum von dreizehn Jahren liegt und die Messung der beiden Zielvariablen über Einzelindikatoren geschah, so dass keine Messfehlerkorrektur vorgenommen werden konnte.

38 Der Befund, dass die Stabilität für den Inglehart-Indexes niedriger ist als jene für das Politikinteresse, widerspricht der Annahme, dass Werte grundsätzlich als sehr veränderungsresistent und persönlichkeitsstrukturell stark verankert gelten. Dieses Ergebnis jedoch kann mit der mangelhaften Validität des Inglehart-Indexes erklärt werden ( vgl. Kapitel 4).
} 
Vergleicht man die hier gemessenen Stabilitätswerte des politischen Interesses mit Resultaten aus der Literatur (Jennings/Stoker 1999, Grob $\left.2006^{39}\right)$, so verfügt die Kohorte der ehemaligen Gymnasiasten über ein sehr stabiles Politikinteresse. Dieser Befund lässt sich Z.T. dadurch erklären, dass die Stabilitätsmessung bei den Befragten im mittleren Erwachsenenalter vorgenommen wurde, einer Lebensphase, in der nach den Ergebnissen von Jennings und Stoker (1999) Politikinteresse stabiler ist als im frühen Erwachsenenalter.

Ein Vergleich der Wertestabilität mit Ergebnissen anderer Paneluntersuchungen, die ebenfalls den Inglehart-Index als Messgrundlage verwenden (z.B. Arzheimer/ Klein 2000, vgl. auch Kapitel 4), zeigt bei der untersuchten Gymnasiastenkohorte ungewöhnlich konstante Werte ${ }^{40}$. Diese hohe Veränderungsresistenz lässt sich möglicherweise auf die Besonderheit der Gymnasiasten-Stichprobe zurückführen. Im Vergleich waren die befragten Gymnasiasten sehr viel postmaterialistischer als die ALLBUSBefragten (vgl. Kapitel 6). Eine leichte Richtungsänderung bei den stark postmaterialistischen Gymnasiasten hin zum Materialismus könnte deshalb auf der nur vierstufigen Inglehart-Skala nicht abbildbar sein.

Gefragt wurde auch nach möglichen Interaktionseffekten bei der Stabilität von Politikinteresse und Wertorientierung. Im Hintergrund stand dabei die Überlegung von Converse (1964), wonach höher gebildete und stärker an Politik interessierte Menschen ein klarer strukturiertes und stabileres Überzeugungssystem besitzen.

Die Überprüfung der Interaktionshypothesen ergibt, dass nur für die Stabilität des Politikinteresses, nicht aber für jene der Wertorientierungen die

\footnotetext{
39 Ein Vergleich mit der Untersuchung von Grob ist nur begrenzt möglich, da der Autor zum einen eine andere Alterskohorte (vom 16. bis zum 35. Lebensjahr) untersucht hat und zudem ein multiples Indikatorenmodell zur Messung des politischen Interesses verwenden konnte (vgl. Grob 2006).

40 Während z.B. Arzheimer und Klein in ihrer Panelanalyse für den Zeitraum zwischen 1994 und 1998 einen Stabilitätskoeffizienten von 0.21 (Somer's D) messen, liegen die Stabilitätswerte hier bei 0.557 bzw. 0.416 (polychorische- bzw. Produktmomentkorrelation) und bei 0.403 bei Verwendung von Somers' $D$.
} 
erwarteten Interaktionseffekte vorliegen. Die 1985 stärker an Politik Interessierten sind demnach deutlich konstanter in ihrem Interesse als ihre politisch weniger interessierten Altersgenossen. Dies bedeutet, dass sich der Personenkreis der weniger Interessierten eher von besonderen Ereignissen oder Geschehnissen - wie Wahlkämpfen oder besonders dramatischen Ereignissen - ,aktivieren' (bzw. deaktivieren) lässt. Im Gegensatz hierzu zeigt die Gruppe der politisch stark Interessierten eine recht konstante Aufmerksamkeit gegenüber politischen Inhalten.

Auch die vom Bildungsniveau der Befragten abhängigen Stabilitätsunterschiede sind hypothesenkonform: Die höhergebildeten Befragten besitzen ein konstanteres Politikinteresse als diejenigen, die ihre Schulkarriere mit Mittlerer Reife oder Abitur beendet haben ${ }^{41}$.

Die zentrale Frage, welche Bedeutung die frühe latente wie manifeste politische Sozialisation auf Politikinteresse und Wertorientierungen im 30. Lebensjahr haben und welche Rolle spätere Erfahrungen dabei spielen, wurde mit Hilfe der Pfadanalyse untersucht. Mit diesem Verfahren ließen sich sowohl die Beziehungen der erklärenden Variablen untereinander als auch direkte wie indirekte Effekte auf die endogenen Variablen zeitgleich untersuchen. Zur Überprüfung der Kausalhypothesen wurden für die jeweiligen Zielvariablen zunächst eigene Kausalmodelle berechnet. Um zu untersuchen, ob innerhalb der Kausalmodelle strukturelle Sozialisationsunterschiede zwischen Männern und Frauen bestehen, wurden anschließend multiple Gruppenvergleiche berechnet.

Vergleicht man die Einflussstärke der verschiedenen Sozialisationseinflüsse auf die beiden Zielvariablen, so lassen sich einige grundsätzliche Ergebnisse festhalten:

\footnotetext{
${ }^{41}$ Da es sich bei der Untersuchungsstichprobe um eine stark bildungsprivilegierte Auswahl handelt, in der nur wenig Varianz im Bildungsniveau vorliegt, dürften die Interaktionseffekte zwischen Bildung und Stabilität der Untersuchungsgegenstände im Bevölkerungsdurchschnitt etwas stärker ausfallen. Außerdem wird die Varianz des Bildungsniveaus zusätzlich durch die überdurchschnittlich starken Panelausfälle bei ,weniger' gebildeten ehemaligen Gymnasiasten weiter reduziert.
} 
- Die politische Sozialisation in der Jugend übt sowohl auf das Politikinteresse als auch auf Wertorientierungen im 30. Lebensjahr einen substanziellen Einfluss aus.

- Die frühen latenten politischen Sozialisationserfahrungen insbesondere in Familie und Schule - sind für die Ausbildung der Wertorientierungen wichtiger als für die Höhe des Politikinteresses.

- Die manifesten politischen Sozialisationseinflüsse während der Jugendzeit hingegen haben einen deutlich stärkeren Einfluss auf das Politikinteresse als auf die Wertorientierung.

- Neben den frühen politischen Sozialisationswirkungen zeigten sich auch sekundäre Sozialisationseffekte. Diese Effekte sind vor allem für die Entwicklung der Wertorientierung relevant. Ihr Einfluss auf die Höhe des Politikinteresses im Erwachsenalter ist etwas geringer.

Für das Politikinteresse ist die Geschlechtzugehörigkeit besonders bedeutsam: Frauen verfügen über deutlich weniger Politikinteresse als Männer. Dies überrascht nicht, da diverse Untersuchungen zur politischen Sozialisation in Jugend bzw. Kindheit belegen, dass Geschlechtsunterschiede in der politischen Interessensausbildung bereits früh sichtbar sind (vgl. z.B. Schmid 2004, Barton/Schäfer 2005, Grob 2006, zur unterschiedlichen Geschlechtersozialisation siehe unten).

Die Untersuchungsergebnisse zur politischen Sozialisation in der Familie zeigen ein verblüffendes Ergebnis. Im Gegensatz zur Hypothese weist das Familienklima als latente Sozialisationserfahrung im Elternhaus einen negativen Einfluss auf: Nicht das positive Familienklima fördert das spätere Interesse an Politik, sondern ganz im Gegenteil das negative. Dieses erstaunliche Ergebnis könnte auf einen generationenspezifischen Effekt hindeuten. So ist denkbar, dass die um 1953/54 geborenen, in einer Zeit des gesellschaftlichen Umbruchs aufwachsenden Jugendlichen in ihren Elternhäusern stärker gegen Autoritätsverhältnisse opponierten. Ein solches Aufbegehren sollte $\mathrm{zu}$ einer intensiveren Auseinandersetzung mit gesellschaftlichen Strukturen führen und damit Politikinteresse fördern. Diese Interpretation wird gestützt durch den ebenfalls negativen Effekt des 
Familienklimas auf die manifeste politische Sozialisation in der Schule (SOWI SCHULE).

Der insgesamt stärkste Effekt auf das Politikinteresse im 30. Lebensjahr geht im Kausalmodell von der manifesten politischen Sozialisation in der Schule aus (,SOWI SCHULE'). Dies bedeutet, dass die frühe politische Sozialisation auf die grundsätzliche ,Politisierung' einen erheblichen Einfluss ausübt. Zwei Fragen bleiben jedoch unbeantwortet: Zum einen bleibt offen, ob das Ausmaß der Auseinandersetzung mit gesellschaftlichen oder politischen Themen in der Schule nicht Folge anderer politischer Sozialisationsprozesse ist. So dürften Jugendliche, die aus politisierten Elternhäusern ${ }^{43}$ stammen $^{2}$ oder die mit Freunden politische Diskussionen führen, sich auch in der Schule mit sozialwissenschaftlichen Fächern leichter tun.

Zum anderen kann keine Aussage über den genauen Zeitpunkt einer etwaigen für politische Sozialisation besonders sensiblen Phase gemacht werden, da in der vorliegenden Studie nur ein Messzeitpunkt aus der Jugend vorliegt. Es kann allerdings festgehalten werden, dass die Phase der mittleren Adoleszenz eine bedeutende Rolle im Prozess der politischen Sozialisation spielt.

Unabhängig von der frühen politischen Sozialisation scheint aber auch die spätere Teilnahme an politischen Demonstrationen (PARTIZIP 1985) das Politikinteresse zu fördern ${ }^{44}$. Der Effekt der Demonstrationsteilnahme ist unter Umständen auch deshalb so ausgeprägt, weil in den frühen 80er Jahre vergleichsweise viele politische Demonstrationen in Deutschland stattfanden. Eine solche ,politische Demonstrationskultur' kann die Hemmschwelle zur Teilnahme an solchen Veranstaltungen absenken, so dass die Sozialisationswirkung der politischen Partizipation stärker sichtbar wird.

Bei der Ausbildung der Wertorientierungen spielt die Geschlechtszugehörigkeit - anders als beim Politikinteresse - keine bedeutende Rolle.

\footnotetext{
43 Außerdem ist zu bedenken, dass in den Index ,SOWI SCHULE' auch der Indikator ,Elternmeinung zur sozialwissenschaftlichen Begabung der Kinder' eingeht.

${ }^{44}$ Hier ist allerdings zu beachten, dass die Kausalitätsrichtung des Einflusses nicht geklärt werden kann; vgl. die Ausführungen im Kapitel 7.
} 
Ebenfalls hypothesenkonform fallen die langfristigen Effekte der latenten politischen Sozialisation in Familie und Schule aus. Dabei ist der Einfluss der elterlichen Erziehungsstile besonders hervorzuheben. Eine Erziehung zu Selbstständigkeit fördert demnach die Ausbildung postmaterialistischer Wertorientierungen. Allerdings ist davon auszugehen, dass das Konstrukt ,Erziehung zur Selbstständigkeit' nicht nur die Erziehungspraktiken im Elternhaus, sondern auch die Wertorientierungen der Eltern misst. Insbesondere das im Konstrukt enthaltenen Item ,Erziehung zur Kritikfähigkeit' dürfte von postmaterialistischen Eltern favorisiert werden. Es lässt sich deshalb nicht klären, in welchem Masse elterliche Erziehung die späteren Wertorientierungen der Kinder beeinflusst bzw. wie stark die Eltern ihre Werte erfolgreich an die Kinder weitergeben. Eine Trennung der beiden inhaltlich miteinander verknüpften Aspekte ,Erziehungspraktiken' und ,Wertorientierungen der Eltern' ist hier nicht möglich.

Die politische Sozialisation nach der mittleren Adoleszenz (BILDUNG 1985 ${ }^{45}$, PARTIZIP 1985, DAUER ERWERB 1985) wirkt den hier berechneten Kausalmodellen zufolge auf die Wertorientierungen deutlich stärker als auf das Politikinteresse. Dies gilt auch für die Höhe des erreichten Bildungsniveaus, d. h. höher Gebildete verfügen - selbst in der im Ganzen sehr bildungsstarken Stichprobe der ehemaligen Gymnasiasten - über ein höheres Maß an postmaterialistischen Werthaltungen als weniger Gebildete. Das Ergebnis, dass der Bildungseffekt hier noch belegbar ist, unterstreicht grundsätzlich die Bedeutung der Bildung für die Entwicklung von Wertorientierungen - zumindest der Inglehartschen Lesart (vgl. Kapitel 4). Dass die Teilnahme an Demonstrationen mit stärker postmaterialistischen Werthaltungen verknüpft ist, dürfte ebenfalls nicht verwundern; insbesondere deshalb, weil eines der postmaterialistischen Items explizit auf ,freie Meinungsäußerung' verweist ${ }^{46}$. Interessanterweise spielen

\footnotetext{
45 Wobei der endgültige Bildungsabschluss in den meisten Fällen vor dem 30. Lebensjahr erreicht worden sein dürfte.

${ }^{46}$ Siehe hierzu auch die Überlegungen zur Kausalitätsrichtung (Kapitel 7).
} 
Demonstrationserfahrungen, die bis zum 15./16. Lebensjahr ${ }^{47}$ gemacht wurden, keine Rolle für die späteren Wertorientierungen ${ }^{48}$. Dies könnte zum einen daran liegen, dass die Beteiligung ${ }^{49}$ nicht primär politisch motiviert war (z.B. ,Demo als Event'). Es könnte aber auch sein, dass Demonstrationen in diesem Alter einfach keine langfristige Bedeutung haben oder von anderen, späteren Einflüssen überlagert werden.

Ein weiteres wichtiges Ergebnis der Untersuchung sind die z.T. sehr deutlichen Geschlechtsunterschiede in der politischen Sozialisation, die mit Hilfe der multiplen Gruppenvergleiche aufgedeckt wurden.

Die Ergebnisse anderer Untersuchungen, wonach die Zugehörigkeit zum weiblichen Geschlecht zu einer geringeren Ausprägung des politischen Interesses führt (in der gängigen Operationalisierung, vgl. Kapitel 4), wird in der vorliegenden Analyse bestätigt (Abbildung 7.2). Bei postmaterialistischen Wertorientierungen (Abbildung 7.4) fanden sich hingegen keine signifikanten Geschlechtsunterschiede.

Ein Unterschied, der für beide Zielvariablen gilt, betrifft die Bedeutung der jugendlichen kognitiven Kompetenz. Für Frauen führt höhere kognitive Kompetenz in der Jugend sowohl in höherem Maße sowohl zu stärkerem Politikinteresse als auch zu eher zu postmaterialistischen Wertorientierungen.

Bei weiblichen Gymnasiasten bewirkt die im Elternhaus erfahrene Erziehung zur Selbstständigkeit zudem, dass in der Schule häufiger Protesterfahrungen gesammelt werden. Möglicherweise treten diese Mädchen in der Schule selbstbewusster auf und lehnen sich eher gegen die bestehenden Autoritätsstrukturen auf.

\footnotetext{
47 Diese Variable wurde nicht in die Berechnung des Kausalmodells mit aufgenommen, da sich gezeigt hat, dass sie so gut wie keinen Einfluss auf die späteren Wertorientierungen ausübt (vgl. Kapitel 7).

${ }^{48}$ Dieses Ergebnis gilt gleichermaßen für das spätere Politikinteresse.

49 Immerhin fast $26 \%$ der ehemaligen Gymnasiasten verfügen 1969/70 über Demonstrationserfahrungen.
} 
Das Ergebnis korrespondieren mit den Resultaten anderer Untersuchungen, wonach Familieneinflüsse bei der politischen Sozialisation für Mädchen bedeutsamer sind als für Jungen (vgl. Schmid 2004, Grob 2006).

Auch bei der schulischen Sozialisation bestehen Differenzen zwischen den Geschlechtern.

So fördert die manifeste Sozialisation in der Schule (SOWI SCHULE) bei Frauen postmaterialistische Wertorientierungen im Erwachsenenalter stärker als bei Männern. Außerdem liegt bei weiblichen Befragten zusätzlich ein direkter Einfluss der schulischen Sozialisationserfahrung auf die Werthaltungen im Alter von 43 Jahren vor. Über die sich hinter diesem Effekt verbergenden Ursachen lassen sich nur Vermutungen anstellen. So könnte es z.B. sein, dass bei Frauen, die sich in der Schule stärker mit sozialwissenschaftlichen Inhalten auseinandergesetzt haben, die zunehmende Lebenserfahrung zu stärker egalitären oder emanzipatorischen Rollenvorstellungen führt und sich dies später in vermehrt postmaterialistischen Wertorientierungen manifestiert.

Ein auffälliger Geschlechtsunterschied zeigt sich auch beim Einfluss der Erwerbstätigkeit auf die Wertorientierungen. Für Frauen hat die Dauer der Erwerbstätigkeit so gut wie keinen Einfluss auf die Wertorientierungen. Bei Männern hingegen wirkt die Erwerbstätigkeit negativ auf die Werthaltungen, d.h. mit Dauer der Berufstätigkeit werden Männer zunehmend materialistisch. Dieser Befund könnte mehrere Ursachen haben: Zum einen ist denkbar, dass Frauen weniger durch Berufserfahrung sozialisiert werden, da sie häufiger in Teilzeit arbeiten und somit weniger Zeit am Arbeitsplatz verbringen. Eine weitere Ursache könnte in der Art der Beschäftigung liegen: Während Frauen häufiger in sozialen Berufen tätig sind - etwa im Bildungssektor oder in pflegerischen Bereichen - arbeiten Männer öfter in technischen und administrativen Berufen. Es ist auch möglich, dass bei Männern - anders als bei Frauen - die Dauer der Berufstätigkeit stärker mit der Aufgabe, Familie und Kinder zu versorgen, verknüpft ist (der Mann als ,Haupternährer'); die Ausbildung materialistischer Wertorientierungen bei Männern könnte auf diese Weise durchaus befördert werden. 
Darüber hinaus ist noch ein weiterer geschlechtsspezifischer Unterschied hervorzuheben: Die Varianzaufklärung gelingt für Frauen bei beiden Zielvariablen besser als für Männern. Dieser Befund kann möglicherweise damit begründet werden, dass für beide Geschlechter die jeweiligen Sozialisationsinstanzen von unterschiedlicher Bedeutung sind. So könnte es beispielsweise sein, dass für Frauen Familie und Schule bedeutungsvollere Sozialisationskontexte darstellen, während für Männer jugendliche Peerbeziehungen und Medien wichtiger sind. Informationen zu Gleichaltrigenbeziehungen bzw. zu Medienkontakten konnten in dieser Untersuchung jedoch nicht berücksichtigt werden.

Inwieweit die beschriebenen Panelausfälle zu einer Einschränkung in der Verallgemeinerung der Ergebnisse führen, lässt sich nicht genau abschätzen. Da relevante Panelausfälle insbesondere bei weniger stark gebildeten Befragten zu verzeichnen waren, werden die Ergebnisse der Bildungseinflüsse möglicherweise unterschätzt. Die anderen Schätzergebnisse sollten durch die Panelausfälle aber nicht wesentlich beeinträchtigt sein. 
Die zentralen Ergebnisse der vorliegenden Arbeit lassen folgende Schüsse zu:

1. Das hohe Maß an Stabilität der Zielvariablen und die gefundenen Sozialisationseffekte aus der Jugend belegen die Existenz langfristiger ,formativer' Einflüsse vor dem Erwachsenenalter. Bis zur mittleren Adoleszenz finden demnach wesentliche politische Sozialisationsprozesse statt.

2. Neu an der Untersuchung ist vor allem die Frage nach der langfristigen Relevanz latenter Sozialisationserfahrungen für die politische Identitätsausbildung. Es konnte gezeigt werden, dass jugendliche latente Sozialisationserfahrungen einen langfristigen Einfluss auf Politikinteresse und insbesondere auf Wertorientierungen ausüben.

Wie im Kapitel 6 dargestellt, wurde die verwendete Panelstudie ursprünglich zur Untersuchung von Bildungs- und Berufverläufen ehemaliger Gymnasiasten entwickelt. Die Tatsache, dass mit den vorliegenden Daten trotz der nur sehr eingeschränkten Operationalisierungsmöglichkeiten latente Sozialisationseinflüsse identifiziert werden konnten, die auch fünfzehn Jahre nach der Primärbefragung noch deutlich wirksam sind, spricht dafür, dass diesen Faktoren eine erhebliche Bedeutung für die politische Identitätsbildung zukommt. Es ist zu vermuten, dass frühe latente Sozialisationserfahrungen auch in anderen Bereichen grundsätzlicher politischer Orientierungen wie Parteiidentifikation, Toleranz und Vorstellungen zur Gleichstellung oder Gerechtigkeit einen langfristigen Einfluss ausüben.

Da insbesondere familiale latente Sozialisationserfahrungen schon geraume Zeit bevor die kognitiven Voraussetzungen zur politischen Reflexion ausgebildet werden wirksam sind, sollte die künftige Forschungspraxis mit der Untersuchung politischer Sozialisationsprozesse nicht erst im Jugendalter beginnen, sondern bereits im Kindesalter einsetzen. Dass für solche langfristigen Untersuchungen früherer (aber auch späterer) Sozialisationserfahrungen Längsschnittstudien vonnöten sind, welche 
Informationen zu den wesentlichen Sozialisationsinstanzen enthalten, versteht sich von selbst.

Im übrigen wäre es wünschenswert, wenn latente wie manifeste Sozialisationseinflüsse über mehrere Indikatoren erhoben würden, so dass eine Messfehlerkorrektur vorgenommen werden kann.

3. Die Tatsache, dass neben den frühen Sozialisationseinflüssen auch Sozialisationserfahrungen nach der Jugend von Bedeutung sind zeigt, dass der Prozess der politischen Sozialisation mit der Jugend nicht abgeschlossen ist.

Für die theoretische Einordnung dieser Ergebnisse in die zeitlichen Modelle des politischen Lernens (Kapitel 2) kommen alle drei Modelle in Betracht: Für das ,Primacy Principle'-Modell sprechen die gefundenen Effekte der latenten politischen Sozialisation in der Familie, da diese nicht erst in der Jugend, sondern bereits in der Kindheit wirksam sein dürften. Die sehr deutlich sichtbaren, schulischen Sozialisationseinflüsse weisen dagegen auf das ,Intermediate Period'-Modell hin. Als Beleg für das ,Recency'-Modell schließlich können die ermittelten Einflüsse nach der jugendlichen Sozialisationsphase gewertet werden. Insgesamt kann also der Aussage Weissbergs beigepflichtet werden, wonach allen drei Modellen ein spezifischer Erklärungswert zukommt, da sie jeweils bestimmte Aspekte des politischen Lernens aufgreifen ${ }^{50}$ (Weissberg 1974: 27, vgl. Kapitel 2).

4. Als weiteres wesentliches Ergebnis sind die z.T. gravierenden Geschlechtsunterschiede in der politischen Sozialisation zu nennen.

Die stärkeren frühen Sozialisationseffekte bei Frauen zeigen, dass für weibliche Gymnasiasten formative Einflüsse eine größere Rolle spielen als für Männer.

Diese Ergebnisse verweisen darauf, dass bei den Geschlechtern im politischen Lernprozess unterschiedliche Mechanismus wirksam sind. Es kann vermutet

\footnotetext{
50 Dies gilt, sofern man die drei Modelle nicht als sich ausschließende Ansätze betrachtet, sondern eine weit gefasste Sichtweise anlegt.
} 
werden, dass diese Geschlechtsunterschiede zum großen Teil in den speziellen politischen Lernprozessen dieser Kohorte begründet liegen.

Um dies zu untersuchen, wäre aber eine eigens für diese Fragestellung entworfene, langfristig angelegte Untersuchung erforderlich. 


\section{Literaturverzeichnis}

Abramson, P.R., (1979): Developing Party Identification: A Further Examination of Life-Cycle, Generational, and Period Effects, in: American Journal of Political Science 23: 78-96.

Ackermann, P. (1996): Das Schulfach ,Politische Bildung' als institutionalisierte politische Sozialisation, in: Claußen, B. und R. Geißler (Hrsg.): Die Politisierung des Menschen. Instanzen der politischen Sozialisation. Ein Handbuch, Opladen, S. 91-100.

Ackermann, P. (2002): Politische Sozialisation: Schule, in: Greiffenhagen, M. und S. Greiffenhagen, (Hrsg.): Handwörterbuch zur politischen Kultur der Bundesrepublik Deutschland, Wiesbaden, S. 450-453.

Adelson, J. (1980): Die politischen Vorstellungen des Jugendlichen in der Frühadoleszenz, in: Döbert, R. (Hrsg.): Entwicklung des Ichs, Königstein, S. 272-293.

Adorno T.W., Frenkel-Brunswik, E., Levinson, D.J., und R.N. Sanford (1950/1969): The Authoritarian Personality, New York.

Allerbeck, K. und W. Hoag (1985): Jugend ohne Zukunft? Einstellungen, Umwelt, Lebensperspektiven, München.

Almond, G. A. und S. Verba (1989): The Civic Culture. Political Attitudes and Democracy in Five Nations, Newbury Park.

Almond, G. A. und S. Verba (1965): The Civic Culture. Political Attitudes and Democracy in Five Nations, Princeton.

Almond, G. A. und S. Verba (1980): The Civic Culture Revisited. An Analytic Study, Princeton.

Alt, C., Teubner, M. und U. Winklhofer (2005): Partizipation in Familie und Schule - Übungsfeld der Demokratie, in: Aus Politik und Zeitgeschichte, Heft 41, S. 24-31.

Amthauer, R. (1953): Intelligenz-Struktur-Test, 2. erweiterte Auflage, Göttingen.

Arzheimer, K. (2005): Gesellschaftliche Wertorientierungen, Staatszielvorstellungen und Ideologien, in: Falter, J., Gabriel, O.W. und H. Rattinger (Hrsg.): Wächst zusammen, was zusammen gehört? Baden-Baden.

Arzheimer, K. und M. Klein (2000): Gesellschaftspolitische Wertorientierungen und Staatszielvorstellungen im Ost-West-Vergleich, in: Falter, J., Gabriel, O.W. und H. Rattinger (Hrsg.): Wirklich ein Volk? Die politischen Orientierungen von Ost- und Westdeutschen im Vergleich, Opladen, S. 363-402. 
Barnes, S. H., Kaase M. und K. Allerbeck (Hrsg., 1979): Political Action, Mass Participation in Five Western Democracies, Beverly Hills.

Becker, R. (2002): Wahlbeteiligung im Lebensverlauf. A-P-K-Analysen für die Bundesrepublik Deutschland in der Zeit von 1953 bis 1987, Kölner Zeitschrift für Soziologie und Sozialpsychologie, Jg. 54, Nr. 2, S. 246-263.

Becker, R. und A. Mays (2003): Soziale Herkunft, politische Sozialisation und Wählen im Lebensverlauf, in: Politische Vierteljahresschrift, 44, S. 19 - 40.

Berelson, B., Lazarsfeld, P. und W. McPhee (1954): Voting, Chicago.

Berton, M. und J. Schäfer (2005): Politische Orientierungen von Grundschulkindern. Ergebnisse von Tiefeninterviews und Pretests mit 6- bis 7-jährigen Kindern, Mannheimer Zentrum für Europäische Sozialforschung, Working Papers Nr. 86.

Birkelbach, K. (1998): Befragungsthema und Panelmortalität: Ausfälle in einer Lebenslauferhebung, in: ZA-Informationen 42, S. 128-147.

Birkelbach, K., Hellwig, J. O., Hemsig, W. und H. Meulemann (2000): Lebenserfolg und Erfolgsdeutung im frühen Erwachsenenalter. Eine Wiederbefragung ehemaliger Gymnasiasten im 43. Lebensjahr, Projektbericht zur Vorlage bei der DFG.

Bollen, K. A. (1989): Structural equations with latent variables, New York.

Bonfadelli, H. (1981): Die Sozialisationsperspektive in der Massenkommunikationsforschung, Berlin.

Bortz, J. (2005): Statistik für Sozialwissenschaftler, Berlin.

Brauer, J.(2006): Das politische Interesse weiblicher Jugendlicher, Potsdam.

Brettschneider, F. van Deth, J. W. und Roller, E. (Hrsg.) (2002): Das Ende der politisierten Sozialstruktur? Opladen.

Buhl, M. (2003): Jugend, Familie, Politik. Familiale Bedingungen und politische Orientierungen im Jugendalter, Opladen.

Bürklin, W. (1988): Wählerverhalten und Wertewandel, Opladen.

Bürklin, W., Klein, M. und A. Ruß (1994): Dimensionen des Wertewandels. Eine empirische Längsschnittanalyse zur Dimensionalität und der Wandlungsdynamik gesellschaftlicher Wertorientierungen, in: Politische Vierteljahresschrift, Bd. 35, S. 579-606.

Bürklin, W. Klein, M. und A. Ruß (1996): Postmaterieller oder anthropozentrischer Wertewandel? Eine Erwiderung auf Ronald Inglehart und Hans-Dieter Klingemann, in: Politische Vierteljahresschrift, 37, S. 517-536.

Burns, N., Schlozman, K. L. und S. Verba (1997): The Public Consequences of Private Inequality: Family Life and Citizen Participation, The American Political Science Review, Vol. 91, S. 373-389. 
Campbell, A., Converse, P. E., Miller, W. E. und D. E. Stokes (1960): The American Voter, New York.

Campbell, B. A. (1980): A theoretical approach to peer influence in adolescent socialization, in: American Journal of Political Science, 24, S. 324344.

Claggett, W. (1981): Partisan acquisition versus partisan intensity: Life-cycle, generation, and period effects, 1952-76, American J ournal of Political Science 25, S. 193-214.

Clarke, H. D., Kornberg A., Mclntyre C., Bauer-Kaase, P. und M. Kaase (1999): The Effect of Economic Priorities on the Measurement of Value Change. New Experimental Evidence, in: American Political Science Review, 93, S. 637-647.

Claußen, B. (1996): Die Politisierung des Menschen und die Instanzen der politischen Sozialisation: Problemfelder gesellschaftlicher Alltagspraxis und sozialwissenschaftlicher Theoriebildung, in: Claußen, B. und R. Geißler (Hrsg.): Die Politisierung des Menschen. Instanzen der politischen Sozialisation, Opladen S. 15-48.

Claußen, B. und R. Geißler (Hrsg.) (1996): Die Politisierung des Menschen. Instanzen der politischen Sozialisation, Opladen.

Converse, P. E. (1964): The Nature of Belief Systems in Mass Publics, in: Apter, D. E. (Hrsg.): Ideology and Discontent. International Yearbook of Political Behavior Research, Bd. 5. New York, S. 206-261.

Dalton, R. J. (1981): The Persistence of Values and Life Cycle Changes, in: Klingemann, H. D., M. Kaase und H. Klaus (Hrsg.): Politische Psychologie. Opladen, S. 189-207.

Dalton, R. J. (1996): Citizen Politics. Public Opinion and Political Parties in Advanced Industrial Democracies, Chatham.

Dawson, R., Prewitt K., und K. Dawson (1977): Political Socialization, Boston.

De Graaf, N. D., Hagenaars, J. und R. Luijkx (1989): Intergenerational Stability of Postmaterialism in Germany, the Netherlands and the United States, in: European Sociological Review, Bd. 5, S. 183-197.

Delli Carpini, M. und S. Keeter (1996): What Americans Know about politics and why it matters, New Haven.

Easton, D. (1965): A Systems Analysis of Political Life, Chicago.

Easton, D. (1975): A Re-Assessment of the Concept of Political Support, in: British J ournal of Political Science, Bd. 5, S. 435-457.

Easton, D. und J. Dennis (1969): A political theory of political socialization, in: Easton, D. und J. Dennis (Hrsg.): Children in the Political System, New York, S. 47-70. 
Engel, U. und J. Reinecke (1994): Panelanalyse, Berlin und New York.

Falter, J., Gabriel, O.W. und H. Rattinger (Hrsg.) (2000): Wirklich ein Volk? Die politischen Orientierungen von Ost- und Westdeutschen im Vergleich, Opladen.

Fend, H. (1991): Identitätsentwicklung in der Adoleszenz. Entwicklungspsychologie der Adoleszenz in der Moderne, Bd.2, Bern.

Fend, H. (2006): Entwicklungspsychologie des Jugendalters. Ein Lehrbuch für pädagogische und psychologische Berufe, Opladen.

Festinger, L. (1957): A Theory of Cognitive Dissonance, Stanford.

Flanagan, S. C. (1982): Measuring Value Change in Advanced Industrial Societies. A Rejoinder to Inglehart, in: Comparative Political Studies Vol. 15, S. 99-127.

Flanagan, S. C., (1979): Value Change and Partisan Change in Japan. The Silent Revolution Revisited, in: Comparative Politics, 11, S. 253-278

Flanagan, S. C., (1987): Value Change in Industrial Societies, in: American Political Science Review, 81, S. 1303-1319.

Fogt, H. (1982): Politische Generationen. Empirische Bedeutung und theoretisches Modell, Opladen.

Franklin, C. H. (1984): Issue Preferences, Socialization, and the Evolution of Party Identification, in: American J ournal of Political Science 28, S. 459-478.

Frenkel-Brunswik, E. (1996): Studien zur autoritären Persönlichkeit. Ausgewählte Schriften, Graz/Wien.

Fuchs, D. (1989): Die Unterstützung in der Bundesrepublik, in: BergSchlosser, D. und Schissler, J. (Hrsg.): Politische Kultur in Deutschland. Opladen, S. 357-377.

Gabriel, O. W. (1986): Politische Kultur, Postmaterialismus und Materialismus in der Bundesrepublik Deutschland, Opladen.

Gabriel, O. W. und van Deth, J. W. (1995): Political Interest, in: van Deth, J. W. und E. Scarbrough (Hrsg.): Beliefs in Government, Vol. 4, The Impact of Values, London, S. 390-411.

Galston, W. A. (2001): Political knowledge, political engagement, and civic education, in: Annual Review of Political Science, Vol. 4, S. 217-234.

Geißler, R. (1996): Politische Sozialisation in der Familie, in: Claußen, B. und R. Geißler (Hrsg.): Die Politisierung des Menschen. Instanzen der politischen Sozialisation, Opladen, S. 51-70.

Geißler, R. (2002): Die Sozialstruktur Deutschlands. Die gesellschaftliche Entwicklung vor und nach der Vereinigung, Opladen. 
Gille, M. (2000): Werte, Rollenbilder und soziale Orientierung, in: Gille, M. und W. Krüger (Hrsg.): Unzufriedene Demokraten. Politische Orientierungen der 16- bis 29jährigen im vereinigten Deutschland, Opladen, S. 143-203

Glenn, N. D. (1977): Cohort Analysis, Beverly Hills.

Glenn, N. D. und T. Hefner, 1972: Further Evidence on Aging and Party Identification, Public Opinion Quarterly 36, S. 31-47.

Greenstein, F. I. (1965): Children and Politics, New Haven.

Greiffenhagen, S. (2002): Politische Sozialisation, in: Greiffenhagen, M. und S. Greiffenhagen (Hrsg.): Handwörterbuch zur politischen Kultur in der Bundesrepublik Deutschland, Wiesbaden, S. 408-418.

Grob, U. (2005): Kurz- und langfristige intergenerationale Transmission von Ausländerablehnung, in: Zeitschrift für Soziologie der Erziehung und Sozialisation, Bd. 25, S. 32-51

Grob, U. (2006): Entwicklung und Stabilität von konventionellem politischem Interesse in langfristiger Perspektive. Ergebnisse aus der LifE-Studie, in: Ittel, A. und H. Merkens, H. Veränderungsmessung und Längsschnittstudien in der empirischen Erziehungswissenschaft, Wiesbaden, S. 61-91.

Hadjar, A. und R. Becker (2006): Bildungsexpansion und Wandel des politischen Interesses in Westdeutschland zwischen 1980 und 2002, in: Politische Vierteljahresschrift, 47. Jg., Heft 1, S. 12-34.

Hagenaars, Jacques A. (1990): Categorical Longitudinal Data. Log-linear panel, trend, and cohort analysis, Newbury Park und London.

Herz, T. A. (1979): Der Wandel von Wertvorstellungen in westlichen Industriegesellschaften, in: Kölner Zeitschrift für Soziologie und Sozialpsychologie, Jg. 31, S. 282-302.

Herz, T. A. (1987): Werte, sozio-politische Konflikte und Generationen, in: Zeitschrift für Soziologie, Jg. 16, Heft 1, S. 56-69.

Hess, R. D. und J. V. Torney (1970): The Development of Political Attitudes in Children, Chicago.

Hoffmann-Lange, U. (2000): Bildungsexpansion, politisches Interesse und politisches Engagement in den alten Bundesländern, in: Niedermayer, O. und B. Westle (Hg.): Demokratie und Partizipation. Opladen, S.46-64.

Hoffmann-Lange, U. (Hrsg.) (1995): Jugend und Demokratie in Deutschland, Opladen.

Holtz-Bacha, C. (1989): Verleidet uns das Fernsehen die Politik? Auf den Spuren der "Videomalaise“, in: Kaase, M. und W. Schulz (Hrsg.), Massenkommunikation. Theorien, Methoden, Befunde, Kölner Zeitschrift für Soziologie und Sozialpsychologie Sonderheft 30, Opladen, S. 239-252. 
Hopf, C. (2000): Familie und Autoritarismus - zur politischen Bedeutung sozialer Erfahrungen in der Familie, in: Rippl, S., Seipel, C. und A. Kindervater (Hrsg.): Kontroversen und Ansätze der aktuellen Autoritarismusforschung, Opladen S. 33-52.

Hopf, C. und W. Hopf (1997): Familie, Persönlichkeit, Politik. Eine Einführung in die politische Sozialisation, Weinheim/München.

Hopf, C., Riecker, P., Sanden-Marcus, M. und C. Schmidt (1995): Familie und Rechtsextremismus, Familiale Sozialisation und rechtsextreme Orientierungen junger Männer, Weinheim-München.

Hurrelmann, K. und D. Ulich (1991): Neues Handbuch der Sozialisationsforschung, Weinheim.

Hurrelmann, K. und M. Albert (2006): Jugend 2006. 15. Shell Jugendstudie. Eine pragmatische Generation unter Druck, Frankfurt.

Hyman, H. (1969): Political Socialization, Glencoe.

Inglehart, R. (1971): The Silent Revolution in Europe. Intergenerational Change in Postindustrial Societies, in: American Political Science Review 65, S. 991-1017.

Inglehart, R. (1977): The Silent Revolution. Changing Values and Political Styles in Western Publics. Princeton.

Inglehart, R. (1979): Wertwandel in den westlichen Gesellschaften: Politische Konsequenzen von materialistischen und postmaterialistischen Prioritäten, in: Klages, H. und P. Kmieciak (Hrsg.): Wertwandel und Gesellschaftlicher Wandel, Frankfurt/Main, S.279-316.

Inglehart, R. (1989): Kultureller Umbruch. Wertewandel in der westlichen Welt. Frankfurt/Main.

Inglehart, R. (1990): Culture Shift in Advanced Industrial Society. Princeton.

Inglehart, R. (1997): Modernisierung und Postmodernisierung. Kultureller, wirtschaftlicher und politischer Wandel in 43 Gesellschaften. Frankfurt/Main und New York.

Ingrisch, M. (1997): Politisches Wissen, politisches Interesse und politische Handlungsbereitschaft bei Jugendlichen in den alten und neuen Bundesländern. Eine Studie zum Einfluss von Medien und anderen Sozialisationsbedingungen, Regenburg.

Jagodzinski, W. (1983): Materialism in Japan Reconsidered: Toward a Synthesis of Generational and Life-Cycle Explanations, in: American Political Science Review, 77, S. 887-894.

Jagodzinski, W. (1984): Identification of Parameters in Cohort Models, in: Sociological Methods \& Research, Bd. 12, S. 375-398. 
Jagodzinski, W. (1985): Die zu stille Revolution - Zum Aggregatswandel materialistischer und postmaterialistischer Wertorientierungen in sechs westeuropäischen Ländern zwischen 1970 und 1981, in: Oberndörfer, D., Rattinger, H. und K. Schmitt (Hrsg.), Wirtschaftlicher Wandel, religiöser Wandel und Wertwandel. Folgen für das politische Verhalten in der Bundesrepublik Deutschland, Berlin, S. 333-356.

Jagodzinski, W. (1996): The Metamorphosis of Life Cycle Change in Longitudinal Studies on Postmaterialism, in: Hyasis, C. und E. K. Scheuch (Hrsg. ), Quantitative Social Research in Germany and Japan, Opladen, S. 2552.

Jagodzinski, W. und S. Kühnel (1987): Estimation of Reliability and Stability in Single-Indicator Multiple-Wave Models Sociological Methods and Research. Bd. 15, S. 219-258

Jagodzinski, W., Kühnel, S. und P. Schmidt (1987): Is there a "Socratic Effect" in nonexperimental panel studies? Consistency of an attitude toward Guestworkers. Sociological Methods and Research 15: 259-302.

Janas, S. und S. Preiser (1999): Politikverdrossenheit bei jungen Erwachsenen, in: Moser, H. (Hrsg.): Sozialisation und Identitäten - Politische Kultur im Umbruch? Sonderheft 99 der Zeitschrift für Politische Psychologie, Bonn, S. 93-119.

Jennings M. K und L. Stoker (2001): The Persistence Of The Past: The Class Of 1965 Turns Fifty, Berkeley.

Jennings, M. K. (1987): Residues of a movement: The aging of the American protest generation, American Political Science Review, Bd. 81, S. 367-82.

Jennings, M. K. und G. B. Markus (1976): The effects of military service on political attitudes: A panel study, in: American Political Science Review, Bd. 71, S. 131-47.

Jennings, M. K. und G. B. Markus (1988): Political involvement in later years: A longitudinal survey, in: American Journal of Political Science, Bd. 32, S. 302-16.

Jennings, M. K. und R. G. Niemi (1968): The transmission of political values from parents to child, in: American Science Review, Bd. 62, S. 169-183.

Jennings, M. K. und R. G. Niemi (1971): The division of political labor between mothers and fathers, in: American Political Science Review, Bd. 65, S. $69-82$

Jennings, M. K. und R. G. Niemi (1981): Generations and Politics, Princeton.

Jennings, M. K. und R.G. Niemi (1974): Political Character in Adolescence, Princeton.

Jennings, M. K., Allerbeck, K. und L. Rosenmayr (1979): Generations and Families. General Orientations, in: Barnes, S., Kaase, M. K. R. Allerback, B. 
Farah, F. Heunks, R. Inglehart, M. K. Jennings, H. D. Klingemann, A. Marsh, L. Rosenmayr: Political Action: Mass Participation in Five Western Democracies, Beverly Hills und London, S. 449-486.

Jennings, M. K., Stoker, L. und J. Bowers (1999): Politics Across Generations: Family Transmission Reexamined, presented at the annual meeting of the American Political Science Association, Atlanta.

Jöreskog, K. G. (1993): Testing structural equation models in: Bollen K. A. und S. J. Long (Hrsg.): Testing structural equation models, Newburry Park, S. 294-316.

Jöreskog, K. G. und D. Sörbom (1988): PRELIS: A preprocessor for LISREL (2nd ed.), in: Scientific Software, Mooresville

Jöreskog, K.G., Sörbom, D., Du Toit, S.H.C. und M. Du Toit (2001): LISREL 8: New Statistical Features, Lincolnwood.

Jugendwerk der Deutschen Shell (Hrsg.) (2002): Jugend 2002, Bd. 1. Frankfurt/ Main.

Jugendwerk der Deutschen Shell (Hrsg.)(1992): Jugend '92, Bd.1, Opladen, S. 213-306.

Kaase, M. (1987): Vergleichende Politische Partizipationsforschung, in: BergSchlosser D., F. Müller-Rommel (Hrsg.): Vergleichende Politikwissenschaft, Opladen, S. 135-150.

Kaase, M. (1989): Politische Einstellungen der Jugend. In: Markefka M., R. Nave-Herz (Hrsg.): Handbuch der Familien- und Jugendforschung, Bd. 2: Jugendforschung, Neuwied und Frankfurt/Main, S. 607-624.

Kandzora, G. (1996): Schule als vergesellschaftete Einrichtung: Heimlicher Lehrplan und politisches Lernen, in: Claußen, B. und R. Geißler (Hrsg.): Die Politisierung des Menschen. Instanzen der politischen Sozialisation, Opladen, S. 71-89.

Klein, M. (1995): Wieviel Platz bleibt im Prokrustesbett? Wertewandel in der Bundesrepublik Deutschland zwischen 1973 und 1992 gemessen anhand des Inglehart-Index, in: Kölner Zeitschrift für Soziologie und Sozialpsychologie, Bd. 47, S. 207-230.

Klein, M. (2005): Gesellschaftliche Wertorientierungen, Wertewandel und Wählerverhalten, in: Falter, J. W. und H. Schoen (Hrsg.): Handbuch Wahlforschung. Wiesbaden, S. 423-446.

Klein, M. (2006): Jugend und politischer Protest, in: Brettschneider, F., van Deth, J. und E. Roller (Hrsg.):Jugend und Politik: „Voll normal“, Wiesbaden, S. 291-317.

Klein, M. und D. Ohr (2004): Ändert der Wertewandel seine Richtung? Die Entwicklung gesellschaftlicher Wertorientierungen in der Bundesrepublik Deutschland zwischen 1980 und 2000, in: R. Schmitt-Beck, Wasmer M., A. 
Koch (Hrsg.): Blickpunkt Gesellschaft 7: Sozialer und politischer Wandel in der Bundesrepublik Deutschland. Analysen mit ALLBUS-Daten aus zwei Jahrzehnten. Opladen, S. 153-178.

Klein, M. und K. Arzheimer (1997): Grau in Grau. Die GRÜNEN und ihre Wähler nach eineinhalb Jahrzehnten, in: Kölner Zeitschrift für Soziologie und Sozialpsychologie, Bd. 49, S. 650-673.

Klein, M. und M. Pötschke (2000): Gibt es einen Wertewandel hin zum „reinen“ Postmaterialismus? Eine Zeitreihenanalyse der Wertorientierungen der westdeutschen Bevölkerung zwischen 1970 und 1997, in: Zeitschrift für Soziologie, Bd. 29, S. 202-216.

Klein, M. und M. Pötschke (2004): Die intra-individuelle Stabilität gesellschaftlicher Wertorientierungen. Eine Mehrebenenanalyse auf der Grundlage des sozio-oekonomischen Panels (SOEP), in: Kölner Zeitschrift für Soziologie und Sozialpsychologie, Bd. 56, S. 432-456.

Kluckhohn, C. (1951): Values and Value Orientations in the Theory of Action. An Exploration in Definition and Classification, in: Parsons, T., E. Shils (Hrsg.), Toward a General Theory of Action. Cambridge, S. 388-433.

Kohlberg, L. (1969): Stage and sequence: The cognitive-developmental approach to socialization, in: Goslin, D. A. (Hrsg): Handbook of Socialization: Theory in Research, Boston and Houghton.

Kohlberg, L. (1976): Collected papers on moral developement and moral education. Cambridge.

Kohn, M. L. (1981): Soziale Schichtung und Eltern-Kind-Beziehungen, in: Lüscher, K. (Hrsg.): Persönlichkeit, Beruf und soziale Schichtung, Stuttgart.

Krampen, G. (1991): Entwicklung politischer Handlungsorientierungen im Jugendalter, Göttingen.

Krampen, G. (1998): Vorhersage politischer Partizipation und Entwicklung politischer Handlungsorientierungen im Übergang vom Jugend- zum frühen Erwachsenenalter, in: Zeitschrift für Entwicklungspsychologie und Pädagogische Psychologie, Bd. 30, S. 80-88.

Krampen, G. und B. Ebel (1991): Zur Bedeutung primärer Bezugspersonen in der politischen Sozialisation von Sekundarstufenschülern, Bd. 18, Universität Trier: Trierer Psychologische Berichte.

Krappmann, L. (1991): Sozialisation in der Gruppe der Gleichaltrigen, in: Hurrelmann, K. und D. Ulich (Hrsg.): Neues Handbuch der Sozialisationsforschung, Weinheim, S. 355-375.

Krimmel, I. (2000): Politische Beteiligung in Deutschland - Strukturen und Erklärungsfaktoren. In: Falter, J. W., Gabriel O. W. und H. Rattinger (Hrsg.): Wirklich ein Volk? Die politischen Orientierungen von Ost- und Westdeutschen im Vergleich. Opladen, S. 609-639. 
Kuhn, H.-P. (2000): Mediennutzung und politische Sozialisation. Eine empirische Studie zum Zusammenhang zwischen Mediennutzung und politischer Sozialisation, Opladen.

Kuhn, H.-P. und H. Oswald (2001): Zur Theorie der Erstwähler, in: Kuhn, H.P., Weiss, K. und H. Oswald (Hrsg.): Jugendliche Wähler in den neuen Bundesländern. Eine Längsschnittstudie zum Verhalten von Erstwählern bei der Bundestagswahl 1998. Opladen, S. 17-36.

Kuhn, H.-P., Weiss, K. und H. Oswald (Hrsg.) (2001): Jugendliche Wähler in den neuen Bundesländern. Eine Längsschnittstudie zum Verhalten von Erstwählern bei der Bundestagswahl 1998. Opladen.

Kühnel, S. und D. Krebs (2007): Statistik für die Sozialwissenschaften, Reinbek.

Kulke, C. (1982): Politische Sozialisation, in: Hurrelmann, K. und D. Ulich (Hrsg.): Neues Handbuch der Sozialisationsforschung, Weinheim, S. 745-776.

Lazarsfeld, P. F., Berelson, B. und H. Gaudet (1944): The People's Choice How the Voter Makes up his Mind in a Presidential Campaign, New York.

Lipset, S. M. und S. Rokkan (1967): Cleavage Structures, Party Systems and Voter Alignments: An Introduction, in: Lipset, S. M. und S. Rokkan (Hrsg.): Party Systems and Voter Alignments. Cross-National Perspectives. New York, S. 1-64.

Maag, G. (1991): Gesellschaftliche Werte. Strukturen, Stabilität und Funktion. Opladen.

Mannheim, K. (1928): Das Problem der Generationen, Kölner Vierteljahreshefte für Soziologie, Bd. 7: 17-76.

Maren O. (2005): Rechtsextremismus unter ost- und westdeutschen Jugendlichen, Einflüsse von gesellschaftlichem Wandel, Familie, Freunden und Schule, Opladen.

Markus, G. B. (1986): Stability and change in political attitudes: Observed, recalled, and 'explained. Political Behavior, Bd. 8, S. 21-44.

Marsh, D. (1971): Political socialization: The implicit assumptions questioned, in: British Journal of Political Science, Bd. 1, S. 453-65.

Maslow, A. H. (1954): Motivation and Personality. New York.

Mason, K. O., Mason, W.M., Winsborough, H. H. und K.W Poole (1973): Some Methodological Issues in Cohort Analysis of Archival Data, in: American Sociological Review, Bd. 38, S. 243-258.

Mayer, K. U. und J. Huinink (1990): Alters-, Perioden- und Kohorteneffekte in der Analyse von Lebensverläufen oder: Lexis Ade?, in: Mayer, K. U. (Hrsg.), Lebensverläufe und sozialer Wandel. Opladen, S. 442-459. 
Melzer, W. (1992): Jugend und Politik in Deutschland: Gesellschaftliche Einstellungen, Zukunftsorientierungen und Rechtsextremismus - Potential Jugendlicher in Ost- und Westdeutschland. Opladen.

Meulemann H. und K. Birklebach (2001): Biographische Erfahrungen und politische Einstellungen zwischen Jugend und Lebensmitte. Die Entwicklung von Wertansprüchen an die Politik bei ehemaligen Gymnasiasten im 16., 30. und 43. Lebensjahr zwischen 1969-1997, in: Politische Vierteljahresschrift 42, S. $30-50$.

Meulemann H., Wieken-Mayser, M. und W. Wiese (1987): Lebensplanung und Lebenserfolg in privilegierten Lebensläufen. Projekt-Endbericht für die Deutsche Forschungsgemeinschaft, Erster Teil: Arbeitsbericht. Köln: Zentralarchiv für empirische Sozialforschung.

Milbrath, L. W. und L. Goel (1977): Political Participation, Chicago.

Mnich, P. (1989): Wertewandel als Kohortenphänomen an Datenmaterial zum Postmaterialismus in der Bundesrepublik von 1974 bis 1986, in: Falter, J. W., Rattinger, H. und K. G. Troitzsch (Hrsg.): Wahlen und politische Einstellungen in der Bundesrepublik Deutschland. Neuere Entwicklungen der Forschung. Frankfurt/Main, S. 263-281.

Neller, K. (2002a): Politische Sozialisation: Massenmedien, in: Greiffenhagen, M. und S. Greiffenhagen (Hrsg.): Handwörterbuch zur politischen Kultur der Bundesrepublik Deutschland, 2. neu bearbeitete Auflage, Wiesbaden, S. 439444.

Neller, K. (2002b): Politisches Interesse, in: Greiffenhagen, M. und S. Greiffenhagen (Hrsg.): Handwörterbuch zur politischen Kultur der Bundesrepublik Deutschland, 2. neu bearbeitete Auflage, Wiesbaden, S. 489494.

Nie, N. H., Junn, J. und K. Stehlik-Barry (1996): Education and Democratic Citizenship in America. Chicago.

Niemi, R. G. und J. Junn, (1998): Civic education: what makes students learn. New Haven und London.

Oerter, R. (1997): Psychologische Aspekte: Können Jugendliche politisch mitentscheiden? In: Palentien, C. und K. Hurrelmann (Hrsg.): Jugend und Politik, Neuwied, S. 32-46.

Oesterreich, D. (1993): Autoritäre Persönlichkeit und Gesellschaftsordnung, Weinheim und München.

Oesterreich, D. (2000): Autoritäre Persönlichkeit und Sozialisation im Elternhaus: Theoretische Überlegungen und empirische Ergebnisse, in: Rippl, S., Seipel, C. und A. Kindervater (Hrsg.): Autoritarismus: Kontroversen und Ansätze der aktuellen Autoritarismusforschung, Opladen, S. 69-90. 
Oesterreich, D. (2002): Politische Bildung von 14-Jährigen in Deutschland. Studien aus dem Projekt Civic Education, Opladen.

Opp, K. D. und P. Schmidt (1995): Einführung in die Mehrvariablenanalyse, Reinbeck.

Oswald, H., Kuhn, H.-P., Rebenstorf, H. und C. Schmid (1999). Brandenburger Jugendlängsschnitt. Universität Potsdam: Institut für Pädagogik, Arbeitspapier B2.

Palentien, C. und K. Hurrelmann (1997): Veränderte Jugend - veränderte Formen der Beteiligung Jugendlicher, in Palentien, C. und K. Hurrelmann (Hrsg.): Jugend und Politik: Ein Handbuch für Forschung, Lehre und Praxis. Neuwied, S. 11-29.

Pawelka, P.(1977): Politische Sozialisation. Wiesbaden.

Piaget, J . (1954/73): Das moralische Urteil beim Kinde. Frankfurt/Main.

Plum, W. (1982): Kohortenanalyse von Umfragedaten. Zur Identifizierung möglicher Einflußfaktoren politischen Verhaltens und politischer Einstellungen, in: Kölner Zeitschrift für Soziologie und Sozialpsychologie, Bd. 34, S. 509-531.

Pöttker, H. (1996): Politische Sozialisation durch Massenmedien: Aufklärung, Manipulation und ungewollte Einflüsse, in: Claußen, B. und R. Geißler (Hrsg.): Die Politisierung des Menschen. Instanzen der politischen Sozialisation. Opladen, S. 149-158.

Rebenstorf, H. (Hrsg.) (2004): Democratic Development? East German, Israeli and Palestinian Adolescents, Wiesbaden.

Rebenstorf, H., Schmid, C. und H.-P. Kuhn (2000): Autoritäre Reaktion und Erziehungsstil - Zur Entwicklung autonomer Persönlichkeit, in: Kuhn, H.-P., Uhlendorff, H. und L. Krappmann (Hrsg.): Sozialisation zur Mitbürgerlichkeit. Opladen, S. 37-58

Reinders, H. (2003): Politische Sozialisation in der Adoleszenz - Eine ReInterpretation quantitativer Längsschnittuntersuchungen in Deutschland, in: Zeitschrift für Entwicklungspsychologie und Pädagogische Psychologie, Bd. 35, S. 98-110.

Reinecke, J. (1999): Interaktionseffekte in Strukturgleichungsmodellen mit der Theorie des geplanten Verhaltens: Multiple Gruppenvergleiche und Produktterme mit latenten Variablen, ZUMA-Nachrichten, Bd. 45, S. 88-114.

Reinecke, J. (2005): Strukturgleichungsmodelle in den Sozialwissenschaften, München.

Rendtel, U. (1995): Lebenslagen im Wandel: Panelausfälle und Panelrepräsentativität, Frankfurt/Main und New York.

Rieker, P. (2007): Fremdenfeindlichkeit und Sozialisation in Kindheit und Jugend, in: Aus Politik und Zeitgeschichte, Heft 37, S. 31-38. 
Riemer, M. (2001): Die Zuverlässigkeit autobiographischer Angaben und die Validität retrospektiv erhobener Lebensverlaufsdaten - sozialkognitive und erhebungstechnische Hintergründe. Max Planck-Institut für Bildungsforschung, Reihe Materialien aus der Bildungsforschung, Berlin.

Rippl S. (2004). Eltern-Kind Transmission. Einflussfaktoren zur Erklärung von Fremdenfeindlichkeit im Vergleich, in: Zeitschrift für Soziologie der Erziehung und Sozialisation, Bd. 24, S. 17-32.

Schmid, C. (2001): Der Einfluss von Eltern und Gleichaltrigen auf das Wahlverhalten von Erstwählern, in: Kuhn, H.-P., Weiss, K. und H. Oswald (Hrsg.): Jugendliche Wähler in den neuen Bundesländern. Eine Längsschnittstudie zum Verhalten von Erstwählern bei der Bundestagswahl 1998. Opladen, S. 151-183.

Schmid, C. (2004): Politisches Interesse von Jugendlichen. Eine Längsschnittuntersuchung zum Einfluss von Eltern, Gleichaltrigen, Massenmedien und Schulunterricht. Wiesbaden.

Schneider, H. (1995): Politische Partizipation - zwischen Krise und Wandel, in: Hoffmann-Lange, U. (Hrsg.): Jugend und Demokratie in Deutschland. Opladen, S. 275-335.

Schulze, G. (1977): Politisches Lernen in der Alltagserfahrung. Eine empirische Analyse. München.

Schulze, G. (1980): Zum Verhältnis von manifester und latenter politischer Sozialisation, in: Schmitt, G. (Hrsg.): Individuum und Gesellschaft in der politischen Sozialisation. Tutzing, S. 71-91.

Sebert, S. K., Jennings, M. K. und R. G. Niemi (1974): The political texture of peer groups, in: Jennings, K., und R. G. Niemi (Hrsg.): The political charakter of adolescense. The influence of families and school. Princeton, S. 229-248.

Statistischen Bundesamt (Hrsg.) (2006): Datenreport 2006, Wiesbaden.

Steinkamp, G. und T. Meyer (1996): Politische Sozialisation durch Arbeitslosigkeit, in: Claussen, B. und R. Geissler (Hrsg.): Die Politisierung des Menschen. Instanzen der politischen Sozialisation. Ein Handbuch. Opladen, S. 321- 338.

Stolle, D. und M. Hooghe (2004): The Roots of Social Capital: Attitudinal and Network Mechanisms in the Relation between Youth and Adult Indicators of Social Capital, Acta Politica, Bd. 39, S.422-441.

Sünker, H. (1996). Informelle Gleichaltrigen-Gruppen im Jugendalter und die Konstitution politischen Alltagsbewusstseins. In Claussen, B. und R. Geißler (Hrsg.): Die Politisierung des Menschen. Instanzen politischer Sozialisation. Ein Handbuch. Opladen, S.101-111. 
Tedin, K. L. (1974): The influence of parents on the political attitudes of adolescents, in: The American Political Science Review, Bd. 68, S. 15791592.

Tedin, K. L. (1980): Assessing peer and parent influence on adolescent political attitudes, in: American Journal of Political Science, Jg. 24, S. 136154.

Torney-Purta, J., Lehmann, R., Oswald, H. und W. Schulz (2001): Citizenship and Education in Twenty-eight Countries: Civic Knowledge and Engagement at Age Fourteen. Amsterdam.

Torney-Purta, J., Oppenheim, A. N. und R. F. Farnen (1975): Civic education in Ten Countries. New York.

Torney-Purta, J., Schwille, J., J.-A. Amadeo (1999): Civic Education across Countries: Twenty-four National Case Studies from the IEA Civic Education Project, Amsterdam.

Treiman, D. J. (1977): Occupational Prestige in Comparative Perspective, New York.

Uehlinger, H.-M. (1988): Politische Partizipation in der Bundesrepublik. Strukturen und Erklärungsmodelle. Opladen.

van Deth, J. W. (1990): Interest in politics, in: Jennings, M. K., van Deth, J. W.: Continuities in political action. Berlin, S. 275-312.

Van Deth, J. W. (1996): Politisches Interesse und Apathie in Europa, in: König, T.; Rieger, E. und H. Schmitt (Hrsg.): Das europäische Mehrebenensystem, Frankfurt, S. 383-402.

Van Deth, J. W. (2000): Das Leben, nicht die Politik ist wichtig, in: Westle, B. und O. Niedermayer (Hrsg.): Demokratie und Partizipation, Wiesbaden, S. 115-400.

van Deth, J. W., 1995: Introduction: The Impact of Values, in: van Deth, J. W. und E. Scarbrough (Hrsg.): The Impact of Values. Oxford, S. 1-18.

Van Deth, Jan W. (1989): Interest in Politics, in : Jennings, M.K., van Deth, J. W. et al.: Continuities in Political Action: A Longitudinal Study of Political Orientations in Three Western Democracies, Berlin und New York, S. 275312.

Verba, S., Burns, N. und K. L. Schlozman (1997): Knowing and Caring about Politics: Gender and Political Engagement, in: Journal of Politics, Bd. 59, S. 1051-1072.

Verba, S., Schlozman, K. L. und H. E. Brady (1995): Voice and equality: Civic volunteerism in American politics. Cambridge.

Vetter, A. (1997): Political Efficacy - Reliabilität und Validität. Wiesbaden. 
Vetter, A. (2000): Frischer Wind in einer alten Beziehung? Political Efficacy und die Bundestagswahlen 1998, in: Falter, J. W., Gabriel, O. W. und H. Rattinger (Hrsg.): Wirklich ein Volk? Die politischen Orientierungen von Ostund Westdeutschen im Vergleich, Opladen, S. 79-109.

Vetter, A. (2006): Jugend: Ein Konzept und seine Messung, in: Roller, E., Brettschneider, F. und J. van Deth, J. (Hrsg.): Jugend und Politik: "Voll normal!", Wiesbaden, S. 25-53.

Wasmund, K. (1982a): Ist der politische Einfluss der Familie ein Mythos oder eine politischen Sozialisation? In: Claußen B. und K. Wasmund (Hrsg.): Handbuch der politischen Sozialisation, Braunschweig, S. 23-63.

Wasmund, K. (1982b): Welchen Einfluss hat die Schule als Agent der politischen Sozialisation? In: Claußen B. und K. Wasmund (Hrsg.): Handbuch der politischen Sozialisation, Braunschweig, S. 64-83.

Wasmund, Klaus (1982c): Was wird wie und wann im Prozess der politischen Sozialisation gelernt? In: Claußen B. und K. Wasmund (Hrsg.): Handbuch der politischen Sozialisation, Braunschweig, S. 143-153.

Watermann, R. (2003): Diskursive Unterrichtsgestaltung und multiple Zielerreichung im politisch bildenden Unterricht. Zeitschrift für Soziologie der Erziehung und Sozialisation, Bd. 23, 356-370.

Watermann, R. und M. Buhl (2003): Zur Einführung in den Themenschwerpunkt: Schule und politische Sozialisation. Zeitschrift für Soziologie der Erziehung und Sozialsation, Bd. 23, S. 340-342.

Weiss, W. (1981): Überlegungen für ein theoretisches Modell politischer Sozialisation, in: Klingemann, H. D. und M. Kaase (Hrsg.): Politische Psychologie. Opladen, S. 37-55.

Weissberg, R. (1974): Political Learning, Political Choice and Democratic Citizenship, Englewood Cliffs.

Weissberg, R. und H. Jacob (1970): Elementary Political Analysis, New York.

Westle B. und H. Schoen (2002): Ein neues Argument in einer alten Diskussion: „Politikverdrossenheit“ als Ursache des gender gap im politischen Interesse, in: Brettschneider, F., van Deth, J. W. und E. Roller (Hrsg.): Das Ende der politisierten Sozialstruktur? Opladen, S. 215-244.

Westle, B. (2006): Politisches Interesse, subjektive politische Kompetenz und politisches Wissen - Eine Fallstudie mit Jugendlichen im Nürnberger Raum, in: Edeltraud R., Brettschneider, F. und J. W. van Deth (Hrsg.): Jugend und Politik: "Voll normal!" - Der Beitrag der Politischen Soziologie zur Jugendforschung, Wiesbaden, S. 209-240.

Zängle, M. (1978): Einführung in die politische Sozialisationsforschung. Paderborn. 
Zinnecker, J. (1985): Jugendliche und Erwachsene. 10. Shell Jugendstudie Opladen.

Zinnecker, J. und A. Fischer (1992): Die wichtigsten Ergebnisse, in: Jugendwerk der Deutschen Shell (Hrsg.): Jugend 92. Lebenslagen, Orientierungen und Entwicklungsperspektiven im vereinigten Deutschland. Opladen, Bd. 1, S. 213 - 282. 